\title{
Unsymmetrically extended polyfused aromatics embedding coronene and perylene frameworks: their syntheses and properties
}

Sushil Kumar ${ }^{1}$, Man-Tzu Ho ${ }^{2}$, Yu-Tai Tao ${ }^{1}$,

${ }^{1}$ Institute of Chemistry, Academia Sinica, Taipei, Taiwan, 115

${ }^{2}$ Department of Chemistry, National Central University, Chung-Li, Taiwan, 32054

\section{Supporting Information}

Table of Contents

Page

1. Schemes, S1-S3 for the synthesis of aryl-derivatives (5-8, 13-17 2-3 and 27-28)

2. Proposed mechanisms for the formation of Ph-BINP and DNC 4-5

3. Experimental Section 6-20

4. $\quad{ }^{1} \mathrm{H}$ and ${ }^{13} \mathrm{C}$ NMR spectra of the derivatives (Figures S1-S74) 21-57

5. MALDI-TOF Mass spectra of final derivatives (Figures S75- 58-63 S86)

6. $\quad$ Single-crystal structures and packing of DBC, Flu-DBC, DNC 64 and Ph-BINP (Figures S87-S88)

7. $\quad{ }^{1} \mathrm{H}$ NMR spectra of Met-BFP recorded at different DMSO-d $\mathrm{d}_{6} \quad 65$ concentrations (Figure S89)

8. Absorption and emission spectra of derivatives in $\mathrm{CH}_{2} \mathrm{Cl}_{2} \quad$ 65-67 (Figures S90-S93)

9. Thermogravimetric plots of DBC and Flu-DBC (Figure S94) 67

10. HOMO/LUMO plots of derivatives (Figure S95) 68

11. $\quad \mathrm{I}_{d} / \mathrm{V}$ curves for single-crystal OFET performance of DBC 69 (Figures S96-S98)

Table S1. Photophysical data of derivatives $\quad 70$

Table S2. Single-crystal OFET data for DBC 70

12. Single-crystal data for the Ph-BINP, DNC, DBC and Flu-DBC 71-89 


\section{Synthetic Schemes, S1-S3 for the synthesis of aryl-derivatives (5-9, 14-18 and}

$\underline{31-34)}$

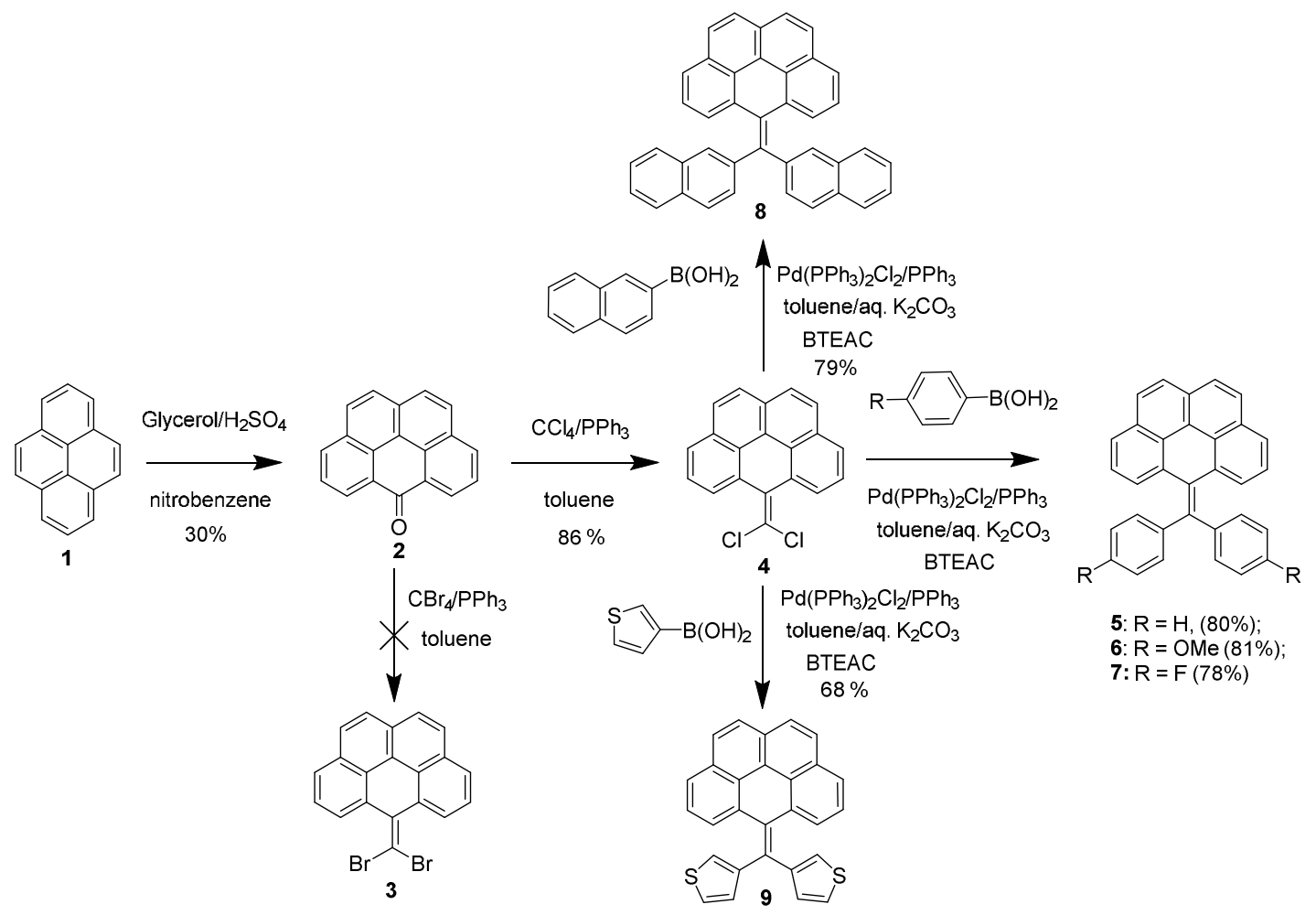

Scheme S1. Synthesis of diaryl-benzo[cd]pyrene derivatives (5-9)

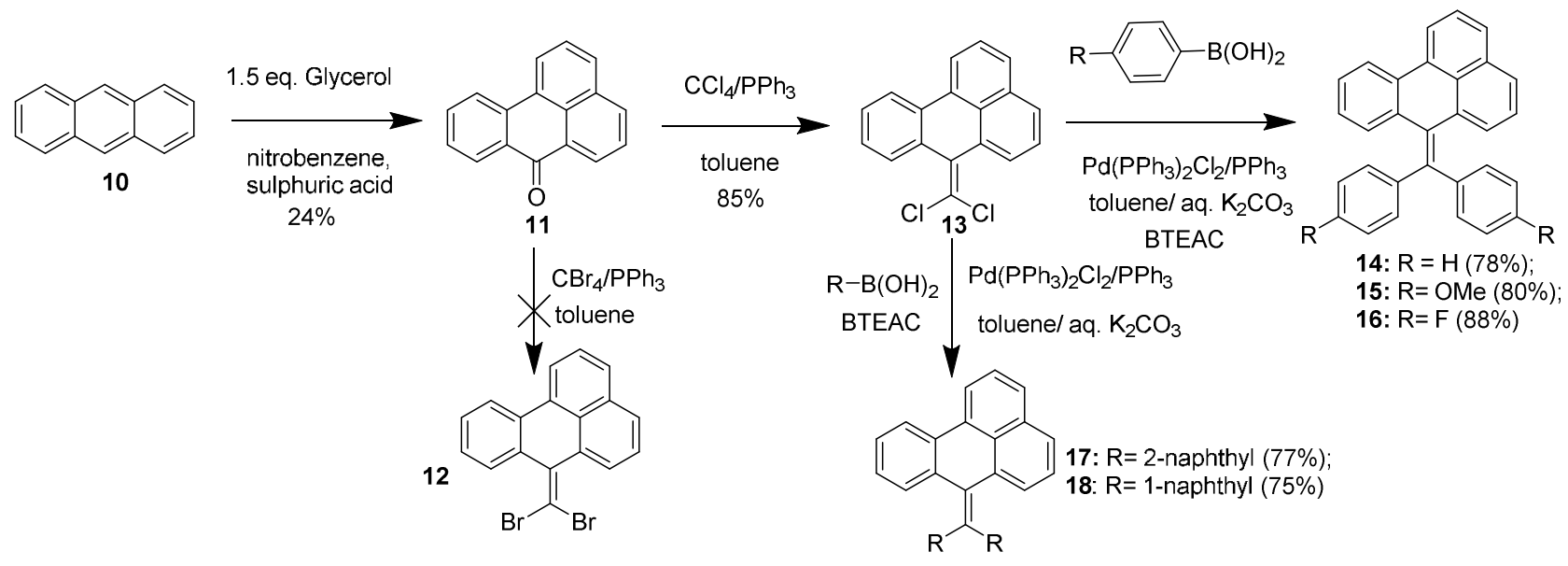

Scheme S2. Synthesis of diaryl-benzo[de]anthracene derivatives (14-18) 


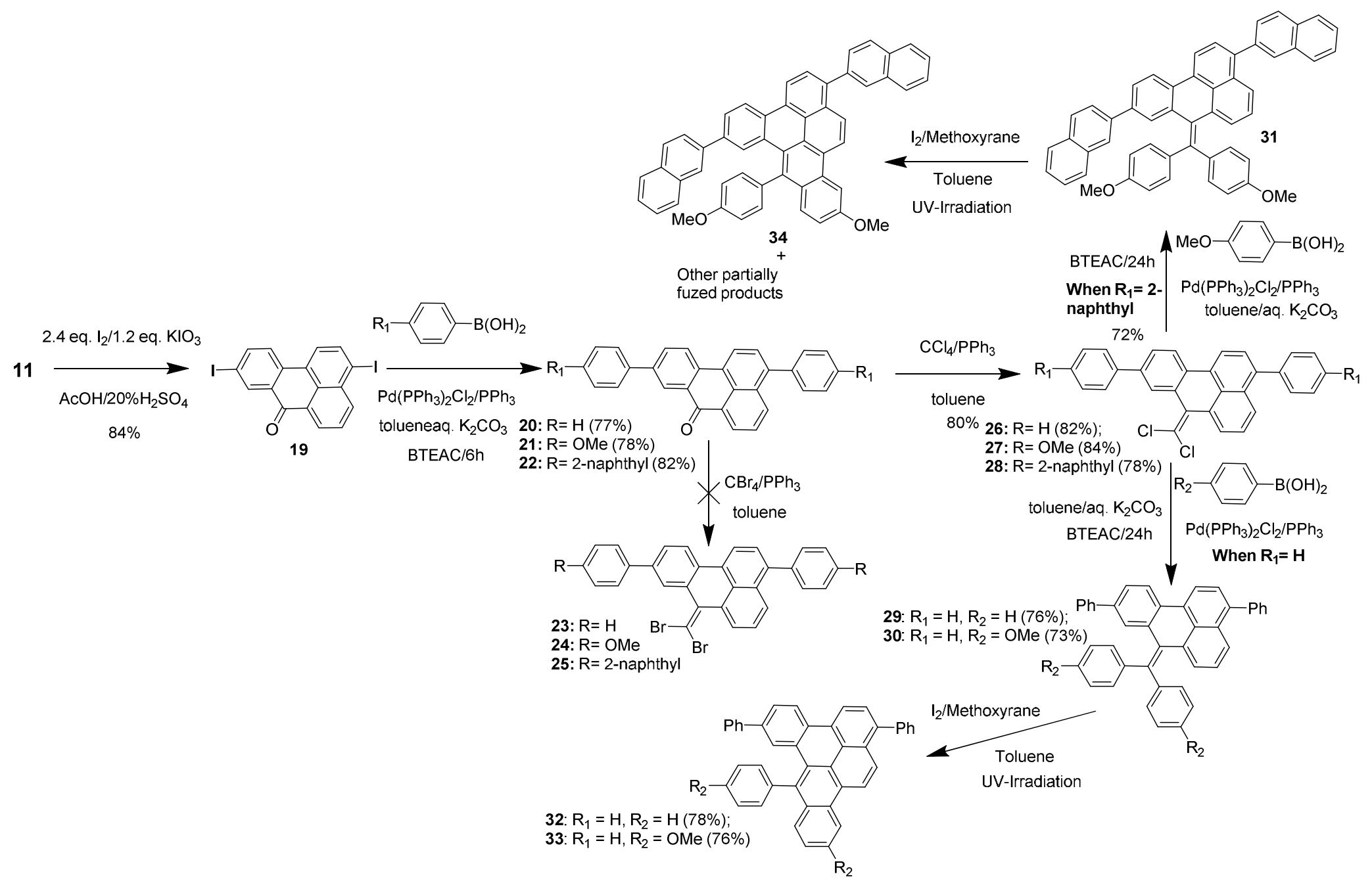

Scheme S3. Synthesis of partially fused derivatives (31-34) 


\section{Suggested mechanisms for the formation Ph-BINP and DNC}

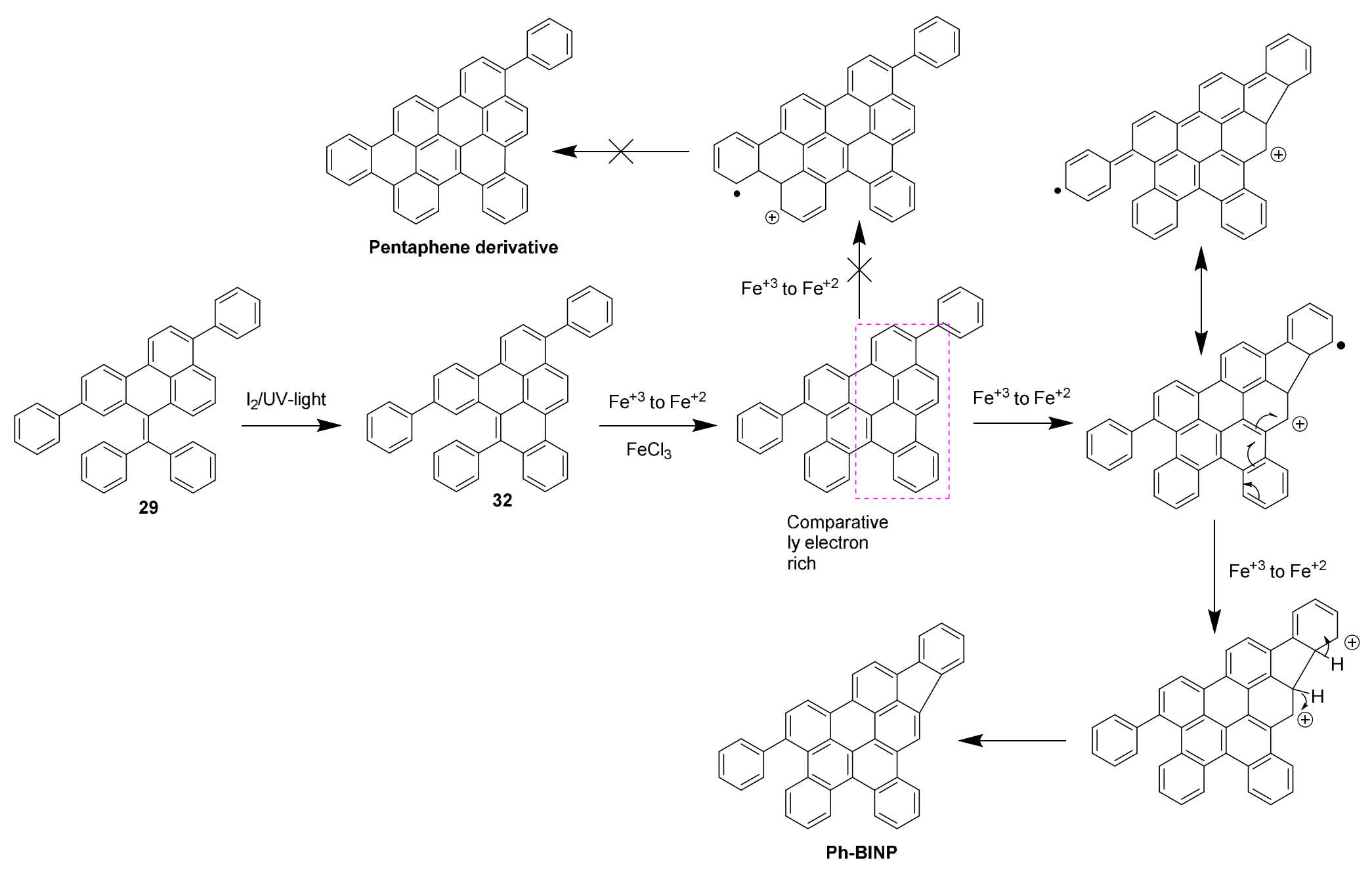

Scheme S4. Mechanism of five-membered cyclodehydrogenation reaction 
Pathway 1.
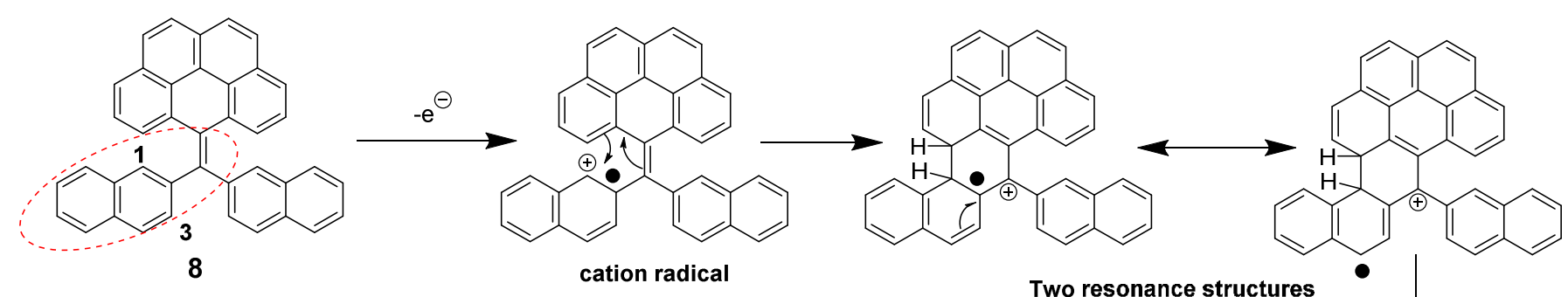

Two resonance structures
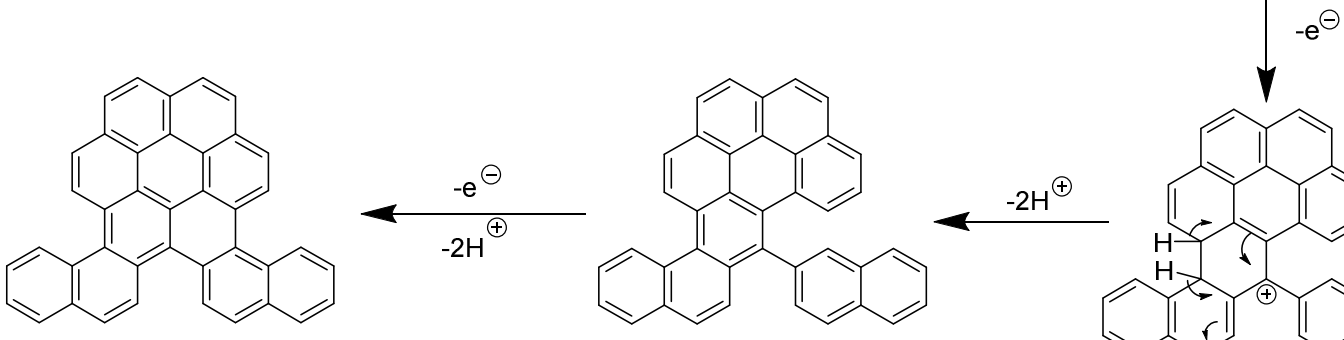

DNC

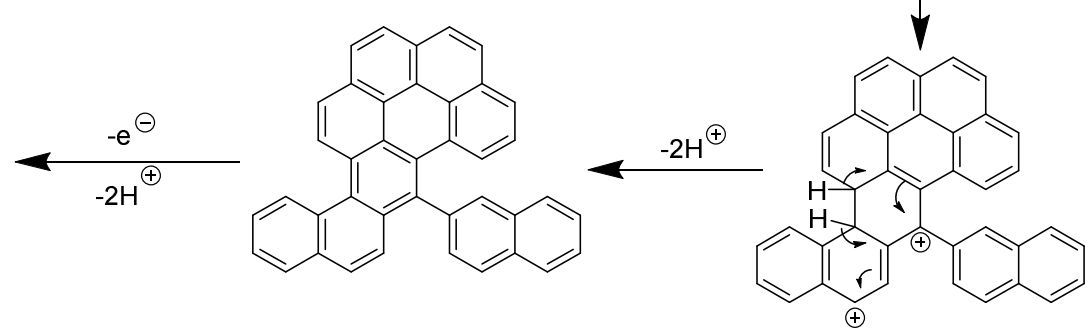

Pathway 2.
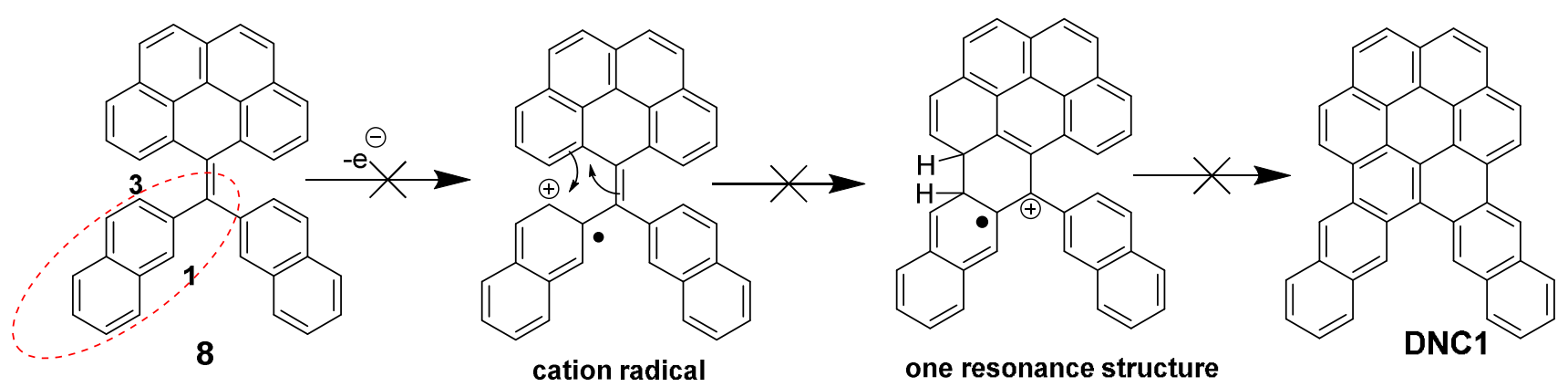

Scheme S5. Mechanism for the formation of DNC 


\section{Supporting Information}

\section{Experimental Section}

Starting materials were purchased from commercial sources. Solvents were distilled and dried prior to use for reaction, spectrophotometric, and spectrofluorimetric analyses. Suzuki couplings, iodine and $\mathrm{FeCl}_{3}$ mediated oxidative reactions were conducted in $\mathrm{N}_{2}$ atmosphere. Starting materials such as $6 H$-benzo[cd]pyren-6-one (2) and $7 \mathrm{H}$ benzo[de]anthracen-7-one (11), 7H-benzo[de]anthracene-7-thione $\quad$ (35) and (diazomethylene)dibenzene (36) were prepared by procedures already reported in literature $^{1-3}$. Column chromatographic separations were performed by use of silica gel with 60-230 mesh size. ${ }^{1} \mathrm{H}$ NMR and ${ }^{13} \mathrm{C}$ NMR were collected on a Bruker AMX400 O FT-NMR spectrometer in chloroform-d or DMSO- $\mathrm{d}_{6}$ with tetramethylsilane (TMS) as the internal standard. V-550JASCO UV/VIS spectrophotometer and HITACHI F-4500 fluorescence spectrophotometer were used to carry out absorption and emission studies in dichloromethane. Thermal decomposition temperatures $\left(\mathrm{T}_{\mathrm{d}}\right)$ of the DBC and Flu-DBC were measured by thermal gravimetric analyzer at a $10{ }^{\circ} \mathrm{C} / \mathrm{min}$ in nitrogen atmosphere. HOMO-LUMO plots of the final derivatives were obtained by PC Spartan Pro software by semi-empirical method at AM1 level.

\section{References}

1. Clar, E.; Mackay, C.C. Tetrahedron 1972, 28, 6041-6047.

2. a) Scheibye, S.; Shabana, R.; Lawesson, S.-O.; Romming, C. Tetrahedron 1982, 38, 993-1002; b) El-Barbary, A. A.; Shabana, R.; Lawesson, S.-O. Phosphorus Sulfur Relat. Elem. 1984, 21, 375-382.

3. Chew, W.; Hynes, R. C.; Harpp, D. N. J. Org. Chem., 1993, 58, 4398-4404.

Preparation of 3,9-diiodo-7H-benzo[de]anthracen-7-one (19) $7 H$-benzo[de]anthracen7-one (11) $(0.23 \mathrm{~g}, 1.0 \mathrm{mmol})$, iodine $(0.61 \mathrm{~g}, 2.4 \mathrm{mmol})$ and potassium iodate $(0.26 \mathrm{~g}$, $1.2 \mathrm{mmol})$ were taken in solvent mixtures of acetic acid $(15 \mathrm{ml})$ and sulphuric acid (2.0 $\mathrm{ml}, 20 \%$ ). The resulting mixture was heated at $90{ }^{\circ} \mathrm{C}$ for $6 \mathrm{~h}$. On completion of the reaction, reaction mixture was cooled. The precipitated product was filtered and washed by methanol. The product was dried and recrystallized from dichloromethane and methanol to afford yellow powder. Yield: $0.40 \mathrm{~g}, 84 \%$. Mp: $281{ }^{\circ} \mathrm{C} .{ }^{1} \mathrm{H}$ NMR $\left(\mathrm{CDCl}_{3}\right.$, $400 \mathrm{MHz}) \delta: 8.81-8.79(\mathrm{~m}, 2 \mathrm{H}), 8.53-8.51(\mathrm{~m}, 1 \mathrm{H}), 8.29$ (d, $J=8.4 \mathrm{~Hz}, 1 \mathrm{H}), 8.09$ (d, $J=$ 


\section{Supporting Information}

8.0 Hz, 1H), 8.05-8.04 (m, 2H), $7.86(\mathrm{t}, J=7.6 \mathrm{~Hz}, 1 \mathrm{H}) .{ }^{13} \mathrm{C}$ NMR could not be recorded due to poor solubility of the derivative. MALDI-TOF MS: calculated for $\left[\mathrm{C}_{17} \mathrm{H}_{8} \mathrm{I}_{2} \mathrm{O}+\mathrm{H}\right]^{+}$: 482.8743, found: 482.8755 .

General procedure for the Suzuki coupling on the 3,9-diiodo-7Hbenzo[de] anthracen-7-one (19) 3,9-diiodo-7H-benzo[de]anthracen-7-one (19) (0.48 g, $1.0 \mathrm{mmol})$, aryl boronic acid (2.5 mmol), $\mathrm{Pd}\left(\mathrm{PPh}_{3}\right)_{2} \mathrm{Cl}_{2}(30 \mathrm{mg}), \mathrm{PPh}_{3}(20 \mathrm{mg})$, potassium carbonate $(1.38 \mathrm{~g}, 10.0 \mathrm{mmol})$ were taken in a mixture of toluene $(50 \mathrm{ml})$ and water $(10$ $\mathrm{ml}$ ). Reaction mixture was heated at $110^{\circ} \mathrm{C}$ for $5 \mathrm{~h}$ in nitrogen atmosphere. On completion of reaction, reaction mixture was cooled and toluene layer was separated. Toluene was removed under vacuum, and product was purified by column chromatography using dichloromethane/hexane as eluent to afford product.

Preparation of 3,9-diphenyl-7H-benzo[de]anthracen-7-one (20) According to general reaction procedure, 3,9-diiodo-7H-benzo[de]anthracen-7-one (19) and phenylboronic acid $(0.26 \mathrm{~g}, 2.5 \mathrm{mmol})$ were reacted to give product as yellow powder. Yield: $0.29 \mathrm{~g}$, 77\%. Mp: $180{ }^{\circ} \mathrm{C} .{ }^{1} \mathrm{H} \mathrm{NMR}\left(\mathrm{CDCl}_{3}, 400 \mathrm{MHz}\right) \delta: 8.84-8.78(\mathrm{~m}, 2 \mathrm{H}), 8.52(\mathrm{~d}, J=7.6 \mathrm{~Hz}$, $1 \mathrm{H}), 8.44(\mathrm{~d}, J=8.4 \mathrm{~Hz}, 1 \mathrm{H}), 8.32$ (d, $J=8.0 \mathrm{~Hz}, 1 \mathrm{H}), 8.01(\mathrm{dd}, J=9.6,2.0 \mathrm{~Hz}, 1 \mathrm{H})$, 7.80-7.72 (m, 3H), $7.66(\mathrm{~d}, J=7.6 \mathrm{~Hz}, 1 \mathrm{H}), 7.56-7.50(\mathrm{~m}, 7 \mathrm{H}), 7.42(\mathrm{t}, J=7.2 \mathrm{~Hz}, 1 \mathrm{H})$. ${ }^{13} \mathrm{C}$ NMR $\left(\mathrm{CDCl}_{3}, 100 \mathrm{MHz}\right) \delta: 183.9,142.7,140.7,139.7,139.6,134.9,133.6,131.7$, $131.3,131.1,130.2,129.8,128.9,128.7,128.4,128.1,127.9,127.8,127.5,128.0,126.5$, 126.1, 125.8, 125.7, 123.7. MALDI-TOF MS: calculated for $\left[\mathrm{C}_{29} \mathrm{H}_{18} \mathrm{O}\right]^{+}:$382.1385, found: 382.1370 .

\section{Preparation of 3,9-bis(4-methoxyphenyl)-7H-benzo[de]anthracen-7-one (21)}

According to general reaction procedure, 3,9-diiodo-7H-benzo[de]anthracen-7-one (19) and (4-methoxyphenyl)boronic acid $(0.38 \mathrm{~g}, 2.5 \mathrm{mmol}))$ were reacted to give product as yellow powder. Yield: $0.34 \mathrm{~g}, 78 \%$. Mp: $167{ }^{\circ} \mathrm{C} .{ }^{1} \mathrm{H}$ NMR $\left(\mathrm{CDCl}_{3}, 400 \mathrm{MHz}\right) \delta: 8.83(\mathrm{~d}$, $J=8.0 \mathrm{~Hz}, 1 \mathrm{H}), 8.73(\mathrm{~d}, J=2.4 \mathrm{~Hz}, 1 \mathrm{H}), 8.50(\mathrm{~d}, J=7.6 \mathrm{~Hz}, 1 \mathrm{H}), 8.41(\mathrm{~d}, J=8.4 \mathrm{~Hz}$, $1 \mathrm{H}), 8.35(\mathrm{~d}, J=8.0 \mathrm{~Hz}, 1 \mathrm{H}), 7.97(\mathrm{dd}, J=7.5,2.4 \mathrm{~Hz}, 1 \mathrm{H}), 7.77-7.72(\mathrm{~m}, 3 \mathrm{H}), 7.63(\mathrm{~d}, J$ $=7.6 \mathrm{~Hz}, 1 \mathrm{H}), 7.49-7.47(\mathrm{~m}, 2 \mathrm{H}), 7.10-7.04(\mathrm{~m}, 4 \mathrm{H}), 3.92(\mathrm{~s}, 3 \mathrm{H}), 3.89(\mathrm{~s}, 3 \mathrm{H}) .{ }^{13} \mathrm{C}$ $\mathrm{NMR}\left(\mathrm{CDCl}_{3}, 100 \mathrm{MHz}\right) \delta: 184.1,159.7,159.5,142.4,140.3,134.5,133.7,132.2,132.1$, 131.6, 131.4, 131.2, 129.8, 128.9, 128.1, 127.6, 126.4, 125.7, 125.5, 123.7, 114.4, 114.0, 55.4. MALDI-TOF MS: calculated for $\left[\mathrm{C}_{31} \mathrm{H}_{22} \mathrm{O}_{3}\right]^{+}: 442.1563$, found: 442.1550 . 


\section{Supporting Information}

Preparation of 3,9-di(naphthalen-2-yl)-7H-benzo[de]anthracen-7-one (22) According to general reaction procedure, 3,9-diiodo-7H-benzo[de]anthracen-7-one (19) and naphthalene-2-boronic acid $(0.43 \mathrm{~g}, 2.5 \mathrm{mmol})$ were reacted to give product as yellow powder. Yield: $0.40 \mathrm{~g}, 82 \%$. Mp: $204{ }^{\circ} \mathrm{C} .{ }^{1} \mathrm{H}$ NMR $\left(\mathrm{CDCl}_{3}, 400 \mathrm{MHz}\right) \delta: 8.92$ (d, $J=2.0$ $\mathrm{Hz}, 1 \mathrm{H}), 8.87$ (d, $J=7.2 \mathrm{~Hz}, 1 \mathrm{H}), 8.57$ (d, $J=7.6 \mathrm{~Hz}, 1 \mathrm{H}), 8.49$ (d, $J=8.4 \mathrm{~Hz}, 1 \mathrm{H}), 8.38$ $(\mathrm{d}, J=8.4 \mathrm{~Hz}, 1 \mathrm{H}), 8.25(\mathrm{~s}, 1 \mathrm{H}), 8.15(\mathrm{dd}, J=10.4,2.0 \mathrm{~Hz}, 1 \mathrm{H}), 8.02-7.92(\mathrm{~m}, 8 \mathrm{H}), 7.78-$ $7.66(\mathrm{~m}, 3 \mathrm{H}), 7.60-7.52(\mathrm{~m}, 4 \mathrm{H}) .{ }^{13} \mathrm{C} \mathrm{NMR}\left(\mathrm{CDCl}_{3}, 100 \mathrm{MHz}\right) \delta: 184.1,142.8,140.8$, 137.2 , 137.0, 135.2, 133.9, 133.7, 133.3, 133.0, 132.8, 132.1, 131.6, 131.3, 130.1, 129.2, $128.9,128.7,128.4,128.2,128.1,128.0,127.8,127.7,126.7,126.5,126.3,126.1,126.0$, 125.1, 123.9. MALDI-TOF MS: calculated for $\left[\mathrm{C}_{37} \mathrm{H}_{22} \mathrm{O}\right]^{+}: 482.1665$, found: 482.1657 .

Preparation of 3',3'-diphenylspiro[benzo[de]anthracene-7,2'-thiirane (37) $7 \mathrm{H}$ benzo[de]anthracene-7-thione $(\mathbf{3 5})(1.23 \mathrm{~g}, 5.0 \mathrm{mmol})$ was dissolved in anhydrous THF $(20 \mathrm{ml})$, and freshly prepared diazomethylenedibenzene (36) (1.36 g, $7.0 \mathrm{mmol})$ solution (in anhydrous THF, $20 \mathrm{ml}$ ) was added to it. The reaction mixture was stirred for $24 \mathrm{~h}$ in nitrogen atmosphere. On completion of reaction, the solvent was removed and product was purified by column chromatography using $\mathrm{dcm} /$ hexane as eluent to give light-yellow solid, $0.56 \mathrm{~g}, 27 \%$. Mp: $256{ }^{\circ} \mathrm{C} .{ }^{1} \mathrm{H}$ NMR $\left(\mathrm{CDCl}_{3}, 400 \mathrm{MHz}\right) \delta: 8.30$ (d, J=7.2 Hz, 1H), $8.05(\mathrm{~d}, J=7.6 \mathrm{~Hz}, 1 \mathrm{H}), 7.87(\mathrm{~d}, J=8.0 \mathrm{~Hz}, 1 \mathrm{H}), 7.67$ (t, $J=7.6 \mathrm{~Hz}, 1 \mathrm{H}), 7.61$ (d, $J=$ 8.0 Hz, 1H), 7.34-7.23 (m, 3H), 7.11-6.74 (m, $J=1.6 \mathrm{~Hz}, 12 \mathrm{H}) .{ }^{13} \mathrm{C} \mathrm{NMR}\left(\mathrm{CDCl}_{3}, 100\right.$ MHz) $\delta: 141.6,141.4,134.9,133.5$ 132.9, 131.6, 129.9, 129.7, 129.5, 129.2, 128.4, $127.6,127.4,127.1,126.9,126.3,126.2,126.0,125.5,123.2,120.0,72.8,60.3$. MALDITOF MS: calculated for $\left[\mathrm{C}_{30} \mathrm{H}_{20} \mathrm{~S}\right]^{+}: 412.1286$, found: 412.1267 .

Preparation of 7-(diphenylmethylene)-7H-benzo[de]anthracene (14) 3',3'diphenylspiro[benzo[de]anthracene-7,2'-thiirane (37) $(0.41 \mathrm{~g}, 1.0 \mathrm{mmol})$ and $\mathrm{PPh}_{3}(0.52$ $\mathrm{g}, 2.0 \mathrm{mmol})$ were taken in toluene $(10 \mathrm{ml})$, and reaction mixture was heated to reflux for $12 \mathrm{~h}$ in nitrogen atmosphere. On completion of reaction, the mixture was cooled and toluene was removed. The product was obtained by column chromatography using dichloromethane/hexane as eluent to afford light-yellow solid. Yield: $0.26 \mathrm{~g}, 69 \%$. Mp: $152{ }^{\circ} \mathrm{C} .{ }^{1} \mathrm{H} \mathrm{NMR}\left(\mathrm{CDCl}_{3}, 400 \mathrm{MHz}\right) \delta: 8.16(\mathrm{~d}, J=7.2 \mathrm{~Hz}, 1 \mathrm{H}), 7.95(\mathrm{~d}, J=8.0 \mathrm{~Hz}$, $1 \mathrm{H}), 7.78(\mathrm{~d}, J=8.0 \mathrm{~Hz}, 1 \mathrm{H}), 7.61-7.56(\mathrm{~m}, 2 \mathrm{H}), 7.24-7.1201(\mathrm{~m}, 13 \mathrm{H}), 7.03$ (t, $J=7.6$ $\mathrm{Hz}, 1 \mathrm{H}), 6.90-6.86(\mathrm{~m}, 1 \mathrm{H}) .{ }^{13} \mathrm{C} \mathrm{NMR}\left(\mathrm{CDCl}_{3}, 100 \mathrm{MHz}\right) \delta: 144.0,143.5,134.6,133.8$, 
$133.5,132.8,132.5,131.2,130.6,129.8,129.7,128.7,128.5,128.3,127.8,127.3,127.1$, $126.5,126.4,125.9,125.8,124.9,123.7,119.6$. MALDI-TOF MS: calculated for $\left[\mathrm{C}_{30} \mathrm{H}_{20}\right]^{+}: 380.1559$, found: 380.1571 .

General method for the preparation of chloromethylene derivatives $(4,13$ and 26-28) Aromatic ketones (2, 11 and 20-22) (10.0 mmol), carbon tetrachloride (4.61 g, 30.0 $\mathrm{mmol})$ and $\mathrm{PPh}_{3}(15.72 \mathrm{~g}, 60.0 \mathrm{mmol})$ were taken in dry toluene $(60 \mathrm{ml})$, and reaction mixture was heated at $100{ }^{\circ} \mathrm{C}$ for $24 \mathrm{~h}$ in nitrogen atmosphere. On completion of reaction, the reaction mixture was cooled and poured into $\mathrm{dcm} / \mathrm{hexane}$ mixture (ratio=1/4; $200 \mathrm{ml}$ ). The solution was filtered through short silica plug. Solvent was removed from the filtrate and product was re-precipitated by dcm/methanol to afford pure compound.

Preparation of 6-(dichloromethylene)-6H-benzo $[\boldsymbol{c d}]$ pyrene (4) According to general reaction procedure, $6 H$-benzo[cd]pyren-6-one (2) (2.54 g, $10.0 \mathrm{mmol})$, carbon tetrachloride and $\mathrm{PPh}_{3}$ were reacted to give product as light-yellow solid. Yield: $2.76 \mathrm{~g}$, 86\%. Mp: $208{ }^{\circ} \mathrm{C} .{ }^{1} \mathrm{H}$ NMR $\left(\mathrm{CDCl}_{3}, 400 \mathrm{MHz}\right) \delta: 8.42$ (d, $\left.J=7.6 \mathrm{~Hz}, 2 \mathrm{H}\right), 7.97-7.90$ (m, $6 \mathrm{H}), 7.69(\mathrm{t}, J=7.6 \mathrm{~Hz}, 2 \mathrm{H}) .{ }^{13} \mathrm{C} \mathrm{NMR}\left(\mathrm{CDCl}_{3}, 100 \mathrm{MHz}\right) \delta: 132.9,130.7,130.2,129.5$, 128.4, 128.1, 127.3, 126.9, 126.4, 125.3, 124.1, 120.7. MALDI-TOF MS: calculated for $\left[\mathrm{C}_{20} \mathrm{H}_{10} \mathrm{Cl}_{2}\right]^{+}: 320.0154$, found: 320.0157 .

Preparation of 7-(dichloromethylene)-7H-benzo[de]anthracene (13) According to general reaction procedure, $7 H$-benzo[de]anthracen-7-one (11) $(2.30 \mathrm{~g}, 10.0 \mathrm{mmol})$, carbon tetrachloride and $\mathrm{PPh}_{3}$ were reacted to give product as light-yellow solid. Yield: 2.52 g, 85\%. Mp: $122{ }^{\circ} \mathrm{C} .{ }^{1} \mathrm{H}$ NMR $\left(\mathrm{CDCl}_{3}, 400 \mathrm{MHz}\right) \delta: 8.17$ (s, $\left.1 \mathrm{H}\right), 8.15(\mathrm{~s}, 1 \mathrm{H}), 8.08-$ $8.06(\mathrm{~m}, 1 \mathrm{H}), 7.98(\mathrm{~d}, J=8.4 \mathrm{~Hz}, 1 \mathrm{H}), 7.86-7.83(\mathrm{~m}, 2 \mathrm{H}), 7.62-7.55$ (m, 2H), 7.47-7.43 (m, 1H), 7.38-7.34 (m, 1H). ${ }^{13} \mathrm{C}$ NMR $\left(\mathrm{CDCl}_{3}, 100 \mathrm{MHz}\right)$ 8: 133.4, 132.9, 132.7, 131.7, 130.3, 129.7, 129.4, 128.4, 128.3, 128.1, 127.7, 127.6, 126.3, 126.2, 125.1, 124.1, 120.4, 119.8. MALDI-TOF MS: calculated for $\left[\mathrm{C}_{18} \mathrm{H}_{10} \mathrm{Cl}_{2}\right]^{+}: 296.0154$, found: 296.0158 .

Preparation of 7-(dichloromethylene)-3,9-diphenyl-7H-benzo[de]anthracene (26) According to general reaction procedure, 3,9-diphenyl-7H-benzo[de]anthracen-7-one (20) $(3.82 \mathrm{~g}, 10.0 \mathrm{mmol})$, carbon tetra chloride and $\mathrm{PPh}_{3}$ were reacted to give product as yellow solid. Yield: $3.68 \mathrm{~g}, 82 \%$. Mp: $170{ }^{\circ} \mathrm{C}$. ${ }^{1} \mathrm{H}$ NMR $\left(\mathrm{CDCl}_{3}, 400 \mathrm{MHz}\right) \delta: 8.34$ (s, $1 \mathrm{H}), 8.22(\mathrm{~d}, J=7.6 \mathrm{~Hz}, 1 \mathrm{H}), 8.18(\mathrm{~d}, J=7.6 \mathrm{~Hz}, 1 \mathrm{H}), 8.07$ (d, $J=8.4 \mathrm{~Hz}, 1 \mathrm{H}), 7.93$ (d, $J=7.6 \mathrm{~Hz}, 1 \mathrm{H}), 7.72-7.70(\mathrm{~m}, 3 \mathrm{H}), 7.58-7.47(\mathrm{~m}, 9 \mathrm{H}), 7.42(\mathrm{t}, J=6.4 \mathrm{~Hz}, 2.0 \mathrm{~Hz}, 1 \mathrm{H})$. 
${ }^{13} \mathrm{C}$ NMR $\left(\mathrm{CDCl}_{3}, 100 \mathrm{MHz}\right) \delta: 140.7,140.4,140.3,138.9,133.1,132.3,132.0,131.0$, 130.2 , 129.8, 129.5, 128.9, 128.3, 128.2, 128.0, 127.9, 127.7, 127.6, 127.5, 127.4, 127.1, 127.0, 126.6, 125.0, 124.6, 120.2, 120.0. MALDI-TOF MS: calculated for $\left[\mathrm{C}_{38} \mathrm{H}_{18} \mathrm{Cl}_{2}\right]^{+}$: 448.0780, found: 448.0793 .

Preparation of 7-(dichloromethylene)-3,9-bis(4-methoxyphenyl)-7Hbenzo[de]anthracene (27) According to general reaction procedure, 3,9-bis(4methoxyphenyl)-7H-benzo[de]anthracen-7-one (21) (4.43 g, $10.0 \mathrm{mmol})$, carbon tetrachloride and $\mathrm{PPh}_{3}$ were reacted to give product as yellow solid. Yield: $4.28 \mathrm{~g}, 84 \%$. Mp: $205{ }^{\circ} \mathrm{C} .{ }^{1} \mathrm{H} \mathrm{NMR}\left(\mathrm{CDCl}_{3}, 400 \mathrm{MHz}\right) \delta: 8.28$ (d, $\left.J=1.6 \mathrm{~Hz}, 1 \mathrm{H}\right), 8.20-8.16(\mathrm{~m}, 2 \mathrm{H})$, $8.04(\mathrm{~d}, J=8.4 \mathrm{~Hz}, 1 \mathrm{H}), 7.50(\mathrm{~d}, J=9.2 \mathrm{~Hz}, 1 \mathrm{H}), 7.67-7.62-(\mathrm{m}, 3 \mathrm{H}), 7.55-7.45$ (m, 4H), 7.07-7.02 (m, 4H), $3.91(\mathrm{~s}, 3 \mathrm{H}), 3.88(\mathrm{~s}, 3 \mathrm{H}) \cdot{ }^{13} \mathrm{C} \mathrm{NMR}\left(\mathrm{CDCl}_{3}, 100 \mathrm{MHz}\right) \delta: 159.4$, 159.2 , 140.2, 138.5, 133.2, 132.9, 132.6, 131.9, 131.8, 131.2, 131.1, 129.8, 129.2, 128.0, $127.6,127.3,126.6,124.9,124.5,120.1,119.7,114.4,113.8,55.4$. MALDI-TOF MS: calculated for $\left[\mathrm{C}_{32} \mathrm{H}_{22} \mathrm{Cl}_{2} \mathrm{O}_{2}\right]^{+}: 508.0991$, found: 508.1003 .

Preparation of 7-(dichloromethylene)-3,9-di(naphthalen-2-yl)-7Hbenzo[de]anthracene (28) According to general reaction procedure, 3,9-di(naphthalen2-yl)-7H-benzo[de]anthracen-7-one (22) (4.83 g, $10.0 \mathrm{mmol})$, carbon tetra chloride and $\mathrm{PPh}_{3}$ were reacted to give product as yellow solid. Yield: $4.29 \mathrm{~g}, 78 \%$. Mp: $220{ }^{\circ} \mathrm{C} .{ }^{1} \mathrm{H}$ NMR $\left(\mathrm{CDCl}_{3}, 400 \mathrm{MHz}\right) \delta: 8.49(\mathrm{~d}, J=1.6 \mathrm{~Hz}, 1 \mathrm{H}), 8.30(\mathrm{~d}, J=7.2 \mathrm{~Hz}, 1 \mathrm{H}), 8.22(\mathrm{~d}, J$ $=7.2 \mathrm{~Hz}, 1 \mathrm{H}), 8.17-8.15(\mathrm{~m}, 2 \mathrm{H}), 8.02-7.85(\mathrm{~m}, 10 \mathrm{H}), 7.70-7.68(\mathrm{~m}, 2 \mathrm{H}), 7.59-7.53(\mathrm{~m}$, $5 \mathrm{H}) .{ }^{13} \mathrm{C} \mathrm{NMR}\left(\mathrm{CDCl}_{3}, 100 \mathrm{MHz}\right) \delta: 140.8,137.8,137.7,133.8,133.4,133.1,132.8$, $132.7,131.2$, 130.0, 129.0, 128.7, 128.4, 128.3, 128.1, 127.9, 127.8, 127.7, 127.4, 126.7, $126.5,126.3,126.1,125.8,125.3,125.2,124.8,120.3,120.1$. MALDI-TOF MS: calculated for $\left[\mathrm{C}_{38} \mathrm{H}_{22} \mathrm{Cl}_{2}\right]^{+}: 548.1093$, found: 548.1090 .

General reaction procedure of Suzuki coupling reaction on dichloromethylene derivatives $(4,13$ and 26-28) with arylboronic acids Dichloromethylene derivative (1.0 $\mathrm{mmol})$, aryl-boronic acid (2.5 mmol), $\mathrm{Pd}\left(\mathrm{PPh}_{3}\right)_{2} \mathrm{Cl}_{2}(30 \mathrm{mg})$ and $\mathrm{PPh}_{3}(20 \mathrm{mg})$, potassium carbonate $(1.38 \mathrm{~g}, 10.0 \mathrm{mmol})$ and benzyltriethyl ammonium chloride $(0.5 \mathrm{~g}, 2.2 \mathrm{mmol})$ were taken in a mixture of toluene $(20 \mathrm{ml})$ and water $(10 \mathrm{ml})$. The reaction mixture was heated at $110^{\circ} \mathrm{C}$ for $24 \mathrm{~h}$ in nitrogen atmosphere. On completion of reaction the mixture 


\section{Supporting Information}

was cooled, and toluene layer was separated. On removal of toluene the product was purified by column chromatography using dichloromethane/hexane as eluent.

Preparation of 6-(diphenylmethylene)-6H-benzo $[c d]$ pyrene (5) According to general reaction procedure, 6-(dichloromethylene)-6H-benzo $[c d]$ pyrene (4) $(0.32 \mathrm{~g}, 1.0 \mathrm{mmol})$ and phenylboronic acid $(0.26 \mathrm{~g}, 2.5 \mathrm{mmol})$ were reacted to give product as light-yellow solid. Yield: 0.32 g, 80\%. Mp: $235{ }^{\circ} \mathrm{C} .{ }^{1} \mathrm{H}$ NMR $\left(\mathrm{CDCl}_{3}, 400 \mathrm{MHz}\right)$ 8: 7.90 (s, 4H), 7.74$7.72(\mathrm{~m}, 2 \mathrm{H}), 7.48(\mathrm{~d}, J=7.2 \mathrm{~Hz}, 2 \mathrm{H}), 7.25-7.13(\mathrm{~m}, 12 \mathrm{H}) .{ }^{13} \mathrm{C}$ NMR $\left(\mathrm{CDCl}_{3}, 100\right.$ MHz) $\delta: 145.7,144.2,133.5,133.2,130.9$ 129.9, 129.0, 128.8, 128.6, 128.0, 127.2, 126.7, 126.4, 126.2, 125.2. MALDI-TOF MS: calculated for $\left[\mathrm{C}_{32} \mathrm{H}_{20}\right]^{+}: 404.1559$, found: 404.1550 .

Preparation of 6-(bis(4-methoxyphenyl)methylene)-6H-benzo[cd]pyrene

According to general reaction procedure, 6-(dichloromethylene)-6H-benzo[cd]pyrene (4) $(0.32 \mathrm{~g}, 1.0 \mathrm{mmol})$ and (4-methoxyphenyl)boronic acid $(0.38 \mathrm{~g}, 2.5 \mathrm{mmol})$ were reacted to give product as yellow solid. Yield: $0.38 \mathrm{~g}, 81 \%$. Mp: $172{ }^{\circ} \mathrm{C} .{ }^{1} \mathrm{H} \mathrm{NMR}\left(\mathrm{CDCl}_{3}, 400\right.$ MHz) $\delta: 7.88(\mathrm{~s}, 4 \mathrm{H}), 7.72(\mathrm{~d}, J=7.6 \mathrm{~Hz}, 2 \mathrm{H}), 7.48(\mathrm{~d}, J=7.6 \mathrm{~Hz}, 2 \mathrm{H}), 7.18$ (t, $J=8.0$ $\mathrm{Hz}, 2 \mathrm{H}), 6.80(\mathrm{~d}, J=8.8 \mathrm{~Hz}, 4 \mathrm{H}), 6.79-6.76(\mathrm{~m}, 4 \mathrm{H}), 3.78(\mathrm{~s}, 3 \mathrm{H}) .{ }^{13} \mathrm{C} \mathrm{NMR}\left(\mathrm{CDCl}_{3}, 100\right.$ MHz) $\delta: 158.2,145.2,137.0,133.7,133.1,131.2,128.9,128.5,128.0,127.1,126.4$, 126.1, 125.3, 114.1, 55.2. MALDI-TOF MS: calculated for $\left[\mathrm{C}_{34} \mathrm{H}_{24} \mathrm{O}_{2}\right]^{+}:$464.1770, found: 464.1777.

Preparation of 6-(bis(4-fluorophenyl)methylene)-6H-benzo[cd]pyrene (7) According to general reaction procedure, 6-(dichloromethylene)-6H-benzo $[c d]$ pyrene (4) (0.32 g, $1.0 \mathrm{mmol})$ and (4-fluorophenyl)boronic acid $(0.35 \mathrm{~g}, 2.5 \mathrm{mmol})$ were reacted to give product as light-yellow solid. Yield: $0.34 \mathrm{~g}, 78 \%$. Mp: $245{ }^{\circ} \mathrm{C} .{ }^{1} \mathrm{H}$ NMR $\left(\mathrm{CDCl}_{3}, 400\right.$

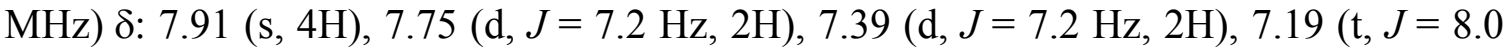
$\mathrm{Hz}, 2 \mathrm{H}), 7.11-7.07(\mathrm{~m}, 4 \mathrm{H}), 6.96-6.91(\mathrm{~m}, 4 \mathrm{H}) .{ }^{13} \mathrm{C} \mathrm{NMR}\left(\mathrm{CDCl}_{3}, 100 \mathrm{MHz}\right) \delta: 162.8$, $160.4,143.1,140.0,134.6,132.8,131.6,131.5,130.9,129.2$, 128.7, $127.8,127.2$, 127.0, 126.3, 125.2, 125.0, 115.8, 115.6. MALDI-TOF MS: calculated for $\left[\mathrm{C}_{32} \mathrm{H}_{18} \mathrm{~F}_{2}\right]^{+}$: 440.1377, found: 440.1370 .

Preparation of 6-(di(naphthalen-2-yl)methylene)-6H-benzo[cd]pyrene (8) According to general reaction procedure, 6-(dichloromethylene)-6H-benzo $[c d]$ pyrene (4) (0.32 g, $1.0 \mathrm{mmol})$ and naphthalene-2-boronic acid $(0.43 \mathrm{~g}, 2.5 \mathrm{mmol})$ were reacted to give 


\section{Supporting Information}

product as yellow solid. Yield: $0.40 \mathrm{~g}, 79 \%$. Mp: $230{ }^{\circ} \mathrm{C} .{ }^{1} \mathrm{H} \mathrm{NMR}\left(\mathrm{CDCl}_{3}, 400 \mathrm{MHz}\right) \delta$ : 7.93-7.92 (m, 4H), 7.80-7.66 (m, 10H), $7.56(\mathrm{~d}, J=7.2 \mathrm{~Hz}, 2 \mathrm{H}), 7.44-7.41(\mathrm{~m}, 4 \mathrm{H}), 7.23$ $(\mathrm{d}, J=8.4 \mathrm{~Hz}, 2 \mathrm{H}), 7.05(\mathrm{t}, J=8.0 \mathrm{~Hz}, 2 \mathrm{H}) .{ }^{13} \mathrm{C} \mathrm{NMR}\left(\mathrm{CDCl}_{3}, 100 \mathrm{MHz}\right) \delta: 145.3$, $141.9,134.1,133.7,133.2132 .1,131.0,129.3,129.1,129.0,128.4,128.2$, 127.9, 127.6, 127.2, 126.9, 126.2, 125.9, 125.8, 125.4, 125.2. MALDI-TOF MS: calculated for $\left[\mathrm{C}_{40} \mathrm{H}_{24}\right]^{+}:$504.1872, found: 504.1876.

Preparation of $3,3^{\prime}-((6 H$-benzo $[c d]$ pyren-6-ylidene)methylene)dithiophene (9) According to general reaction procedure, 6-(dichloromethylene)-6H-benzo $[c d]$ pyrene (4) $(0.32 \mathrm{~g}, 1.0 \mathrm{mmol})$ and thiophene-3-boronic acid $(0.32 \mathrm{~g}, 2.5 \mathrm{mmol})$ were reacted to give product as yellow solid. Yield: $0.28 \mathrm{~g}, 68 \%$. Mp: $196{ }^{\circ} \mathrm{C} .{ }^{1} \mathrm{H} \mathrm{NMR}\left(\mathrm{CDCl}_{3}, 400 \mathrm{MHz}\right) \delta$ : 7.79 (s, 4H), 7.79-7.77 (m, 2H), 7.51 (d, $J=7.2 \mathrm{~Hz}, 2 \mathrm{H}), 7.26$ (t, $J=7.2 \mathrm{~Hz}, 2 \mathrm{H}), 7.20$ $7.18(\mathrm{~m}, 2 \mathrm{H}), 6.99(\mathrm{dd}, J=8.0 \mathrm{~Hz}, 1.2 \mathrm{~Hz}, 2 \mathrm{H}), 6.67-6.65(\mathrm{~m}, 2 \mathrm{H}) .{ }^{13} \mathrm{C} \mathrm{NMR}\left(\mathrm{CDCl}_{3}\right.$, $100 \mathrm{MHz}) \delta: 143.8,134.8,134.3,133.0$ 130.9, 129.1, 128.9, 128.2, 127.7, 127.2, 126.9, 126.1, 125.4, 125.1, 123.7. MALDI-TOF MS: calculated for $\left[\mathrm{C}_{28} \mathrm{H}_{16} \mathrm{~S}_{2}\right]^{+}: 416.0687$, found: 416.0678 .

Preparation of 7-(diphenylmethylene)-7H-benzo[de]anthracene (14) According to general reaction procedure, 7-(dichloromethylene)-7H-benzo[de]anthracene (13) (0.30 g, $1.0 \mathrm{mmol})$ and phenylboronic acid $(0.26 \mathrm{~g}, 2.5 \mathrm{mmol})$ were reacted to give product as light-yellow solid. Yield: $0.30 \mathrm{~g}, 78 \%$.

Preparation of 7-(bis(4-methoxyphenyl)methylene)-7H-benzo[de]anthracene (15) According to general reaction procedure, 7-(dichloromethylene)-7H-benzo[de]anthracene (13) $(0.30 \mathrm{~g}, 1.0 \mathrm{mmol})$ and (4-methoxyphenyl)boronic acid (0.38 g, $2.5 \mathrm{mmol})$ were reacted to give product as yellow solid. Yield: $0.35 \mathrm{~g}, 80 \%$. Mp: $150{ }^{\circ} \mathrm{C} .{ }^{1} \mathrm{H}$ NMR $\left(\mathrm{CDCl}_{3}, 400 \mathrm{MHz}\right) \delta: 8.15(\mathrm{~d}, J=7.2 \mathrm{~Hz}, 1 \mathrm{H}), 7.94(\mathrm{~d}, J=8.0 \mathrm{~Hz}, 1 \mathrm{H}), 7.78(\mathrm{~d}, J=8.0$ $\mathrm{Hz}, 1 \mathrm{H}), 7.61-7.55(\mathrm{~m}, 2 \mathrm{H}), 7.25-7.22(\mathrm{~m}, 3 \mathrm{H}), 7.10-7.06(\mathrm{~m}, 3 \mathrm{H}), 7.03-7.01(\mathrm{~m}, 2 \mathrm{H})$, $6.92(\mathrm{t}, J=7.6 \mathrm{~Hz}, 1 \mathrm{H}), 6.80-6.72(\mathrm{~m}, 4 \mathrm{H}), 3.78(\mathrm{~s}, 3 \mathrm{H}), 3.75(\mathrm{~s}, 3 \mathrm{H}) .{ }^{13} \mathrm{C} \mathrm{NMR}\left(\mathrm{CDCl}_{3}\right.$, $100 \mathrm{MHz}) \delta: 158.1,143.0,136.8,136.2,135.1,133.7,133.1,132.9,131.3,131.1,130.9$, $130.8,128.7,127.8,126.9,126.8,126.3,125.9$, 125.8, 125.1, 123.8, 119.4, 114.0, 113.7, 55.1. MALDI-TOF MS: calculated for $\left[\mathrm{C}_{32} \mathrm{H}_{24} \mathrm{O}_{2}\right]^{+}: 440.1770$, found: 440.1768 .

Preparation of 7-(bis(4-fluorophenylphenyl)methylene)-7H-benzo[de]anthracene (16) According to general reaction procedure, 7-(dichloromethylene)-7H- 


\section{Supporting Information}

benzo[de]anthracene (13) (0.30 g, $1.0 \mathrm{mmol})$ and (4-fluorophenyl)boronic acid (0.35 g, $2.5 \mathrm{mmol}$ ) were reacted to give product as yellow solid. Yield: $0.37 \mathrm{~g}, 88 \%$. Mp: $245{ }^{\circ} \mathrm{C}$. ${ }^{1} \mathrm{H}$ NMR $\left(\mathrm{CDCl}_{3}, 400 \mathrm{MHz}\right) \delta: 8.16(\mathrm{~d}, J=7.2 \mathrm{~Hz}, 1 \mathrm{H}), 7.95(\mathrm{~d}, J=7.6 \mathrm{~Hz}, 1 \mathrm{H}), 7.79$ (d, $J=8.0 \mathrm{~Hz}, 1 \mathrm{H}), 7.65-7.57(\mathrm{~m}, 2 \mathrm{H}), 7.29-7.25$ (m, 1H), 7.18-7.05 (m, 7H), 7.05-6.88 (m,

$5 \mathrm{H}) .{ }^{13} \mathrm{C} \mathrm{NMR}\left(\mathrm{CDCl}_{3}, 100 \mathrm{MHz}\right) \delta: 162.8,160.3,141.0,139.8,139.2,134.6,134.2$, $133.8,132.8,132.2,131.5,131.4,131.3,131.1,130.5,128.5,128.0,127.4,127.2,126.8$, 126.0, 125.0, 123.8, 119.8, 115.7, 115.5, 115.4, 115.2. MALDI-TOF MS: calculated for $\left[\mathrm{C}_{30} \mathrm{H}_{18} \mathrm{~F}_{2}\right]^{+}:$416.1371, found: 416.1379 .

Preparation of 7-(di(naphthalen-2-yl)methylene)-7H-benzo[de]anthracene

According to general reaction procedure, 7-(dichloromethylene)-7H-benzo[de]anthracene (13) $(0.30 \mathrm{~g}, 1.0 \mathrm{mmol})$ and naphthalene-2-boronic acid $(0.43 \mathrm{~g}, 2.5 \mathrm{mmol})$ were reacted to give product as yellow solid. Yield: $0.37 \mathrm{~g}, 77 \%$. Mp: $210{ }^{\circ} \mathrm{C} .{ }^{1} \mathrm{H} \mathrm{NMR}\left(\mathrm{CDCl}_{3}, 400\right.$

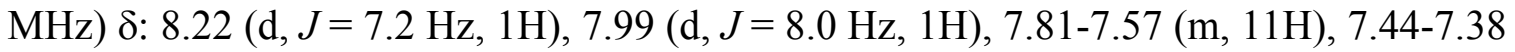
$(\mathrm{m}, 4 \mathrm{H}), 7.36-7.31(\mathrm{~m}, 3 \mathrm{H}), 7.23-7.18(\mathrm{~m}, 2 \mathrm{H}), 6.92(\mathrm{t}, J=8.0 \mathrm{~Hz}, 1 \mathrm{H}), 6.780(\mathrm{t}, J=8.0$ $\mathrm{Hz}, 1 \mathrm{H}) .{ }^{13} \mathrm{C} \mathrm{NMR}\left(\mathrm{CDCl}_{3}, 100 \mathrm{MHz}\right) \delta: 141.7,141.1,134.6,134.1,133.8,133.6,133.4$, $132.9,132.5,132.0,131.3,130.8,129.1,128.9,128.6,128.5,128.3,128.2,128.1,128.0$, $127.9,127.7,127.6,127.5,127.4,127.3,126.7,126.1,126.0,125.9,125.8,125.7,125.2$, 123.8, 119.7. MALDI-TOF MS: calculated for $\left[\mathrm{C}_{38} \mathrm{H}_{24}\right]^{+}: 480.1872$, found: 480.1878 .

Preparation of 7-(di(naphthalen-1-yl)methylene)-7H-benzo[de]anthracene (18) According to general reaction procedure, 7-(dichloromethylene)-7H-benzo[de]anthracene (13) $(0.30 \mathrm{~g}, 1.0 \mathrm{mmol})$ and naphthalene-1-boronic acid ( $0.43 \mathrm{~g}, 2.5 \mathrm{mmol})$ were reacted to give product as yellow solid. Yield: $0.36 \mathrm{~g}, 75 \%$. Mp: $220{ }^{\circ} \mathrm{C} .{ }^{1} \mathrm{H}$ NMR $\left(\mathrm{CDCl}_{3}, 400\right.$

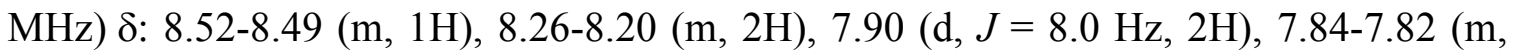
1H), 7.77-7.73 (m, 2H), 7.66-7.57 (m, 4H), 7.50-7.44 (m, 3H), 7.38-7.12 (m, 8H), 6.79 (t, $J=8.0 \mathrm{~Hz}, 1 \mathrm{H}), 6.63(\mathrm{t}, J=7.2 \mathrm{~Hz}, 1 \mathrm{H}) .{ }^{13} \mathrm{C} \mathrm{NMR}\left(\mathrm{CDCl}_{3}, 100 \mathrm{MHz}\right) \delta: 142.1,141.7$, $137.7,136.9,134.9,133.9,133.8,133.3,132.8,132.4,130.9,130.8,128.9,128.7,128.4$, $127.9,127.3$, 127.2, 127.0, 126.7, 126.5, 126.4, 126.2, 126.1, 125.9, 125.8, 125.6, 125.5, 125.4, 125.1, 125.0, 123.5, 119.9. MALDI-TOF MS: calculated for $\left[\mathrm{C}_{38} \mathrm{H}_{24}\right]^{+}: 480.1872$, found: 480.1880 .

Preparation of 7-(diphenylmethylene)-3,9-diphenyl-7H-benzo[de]anthracene (29) According to general reaction procedure, 7-(dichloromethylene)-3,9-diphenyl-7H- 


\section{Supporting Information}

benzo[de]anthracene (26) $(0.45 \mathrm{~g}, 1.0 \mathrm{mmol})$ and phenylboronic acid $(0.26 \mathrm{~g}, 2.5 \mathrm{mmol})$ were reacted to give product as yellow solid. Yield: $0.40 \mathrm{~g}, 76 \%$. Mp: $232{ }^{\circ} \mathrm{C} .{ }^{1} \mathrm{H}$ NMR $\left(\mathrm{CDCl}_{3}, 400 \mathrm{MHz}\right) \delta: 8.23(\mathrm{~d}, J=7.6 \mathrm{~Hz}, 1 \mathrm{H}), 8.04(\mathrm{~d}, J=8.0 \mathrm{~Hz}, 1 \mathrm{H}), 7.70(\mathrm{~d}, J=9.2$ $\mathrm{Hz}, 1 \mathrm{H}), 7.57-7.44$ (m, 8H), 7.37-7.17 (m, 14H), 7.04-6.97 (m, 3H). ${ }^{13} \mathrm{C} \mathrm{NMR}\left(\mathrm{CDCl}_{3}\right.$, $100 \mathrm{MHz}) \delta: 144.5,143.8,143.5,140.7,140.2$, 138.5, 134.5, 133.7, 132.7, 132.5, 131.1, $130.4,130.2,129.8,129.7,129.5,128.9,128.7,128.5,128.4,128.3,127.8,127.3,127.1$, $127.0,126.4,126.3,125.8,124.9,124.1,119.4$. MALDI-TOF MS: calculated for $\left[\mathrm{C}_{42} \mathrm{H}_{28}\right]^{+}: 532.2185$, found: 532.2200 .

Preparation of 7-(bis(4-methoxyphenyl)methylene)-3,9-diphenyl-7Hbenzo[de]anthracene (30) According to general reaction procedure, 7(dichloromethylene)-3,9-diphenyl-7H-benzo[de]anthracene (27) $(0.45 \mathrm{~g}, 1.0 \mathrm{mmol})$ and (4-methoxyphenyl)boronic acid $(0.38 \mathrm{~g}, 2.5 \mathrm{mmol})$ were reacted to give product as yellow solid. Yield: $0.43 \mathrm{~g}, 73 \%$. Mp: $240{ }^{\circ} \mathrm{C}$. ${ }^{1} \mathrm{H}$ NMR $\left(\mathrm{CDCl}_{3}, 400 \mathrm{MHz}\right) \delta: 8.22$ (d, $J$ $=7.6 \mathrm{~Hz}, 1 \mathrm{H}), 8.04(\mathrm{~d}, J=8.0 \mathrm{~Hz}, 1 \mathrm{H}), 7.69(\mathrm{~d}, J=9.2 \mathrm{~Hz}, 1 \mathrm{H}), 7.56-7.44(\mathrm{~m}, 8 \mathrm{H}), 7.35-$ $7.20(\mathrm{~m}, 6 \mathrm{H}), 7.10-7.01(\mathrm{~m}, 5 \mathrm{H}), 6.87-6.76(\mathrm{~m}, 4 \mathrm{H}), 3.78(\mathrm{~s}, 3 \mathrm{H}), 3.77$ (s, 3H). ${ }^{13} \mathrm{C}$ NMR $\left(\mathrm{CDCl}_{3}, 100 \mathrm{MHz}\right) \delta: 158.4,158.1,143.3,140.8,140.0,138.4,137.2,136.1,135.0$, 133.3, 132.9, 132.6, 131.0, 131.0, 130.5, 130.1, 129.4, 129.0, 128.4, 128.2, 127.6, 127.2, 127.0, 125.6, 125.1, 124.7, 124.1, 119.3, 114.2, 113.9, 55.3, 55.1. MALDI-TOF MS: calculated for $\left[\mathrm{C}_{44} \mathrm{H}_{32} \mathrm{O}_{2}\right]^{+}: 592.2396$, found: 592.2384 .

Preparation of 7-(bis(4-methoxyphenyl)methylene)-3,9-di(naphthalen-2-yl)-7Hbenzo[de]anthracene (31) According to general reaction procedure, 7(dichloromethylene)-3,9-di(naphthalen-2-yl)-7H-benzo[de]anthracene (28) (0.55 g, 1.0 mmol) and (4-methoxyphenyl)boronic acid (0.38 g, $2.5 \mathrm{mmol})$ were reacted to give product as yellow solid. Yield: $0.50 \mathrm{~g}, 72 \%$. Mp: $267^{\circ} \mathrm{C} .{ }^{1} \mathrm{H} \mathrm{NMR}\left(\mathrm{CDCl}_{3}, 400 \mathrm{MHz}\right) \delta$ : 8.27 (d, $J=7.6 \mathrm{~Hz}, 1 \mathrm{H}), 8.10$ (d, $J=8.4 \mathrm{~Hz}, 1 \mathrm{H}), 7.99$ (s, 1H), 7.95-7.92 (m, 3H), 7.84$7.65(\mathrm{~m}, 7 \mathrm{H}), 7.61(\mathrm{~d}, J=8.0 \mathrm{~Hz}, 1 \mathrm{H}), 7.57-7.48(\mathrm{~m}, 4 \mathrm{H}), 7.39-7.27$ (m, 5H), 7.11-7.05 (m, 3H), 6.96-6.94 (m, 2H), 6.81-6.79 (m, 2H), 3.81 (s, 3H), 3.78 (s, 3H). ${ }^{13} \mathrm{C}$ NMR $\left(\mathrm{CDCl}_{3}, 100 \mathrm{MHz}\right) \delta$ : 158.3, 158.1, 143.4, 140.0, 138.4, 138.3, 138.2, 137.3, 136.2, 135.0, 133.7, 133.4, 133.2, 132.9, 132.7, 132.6, 132.5, 131.2, 131.1, 130.9, 130.6, 129.8, $129.1,128.8,128.6,128.2,128.1,128.0,127.8,127.7,127.6,127.5,127.4,126.3,126.1$, 


\section{Supporting Information}

$126.0,125.9,125.8,125.4,125.2,124.8,124.2,119.4,114.3,114.0,55.2,55.1$. MALDITOF MS: calculated for $\left[\mathrm{C}_{52} \mathrm{H}_{36} \mathrm{O}_{2}\right]^{+}: 692.2710$, found: 692.2696 .

General procedure for $\mathrm{I}_{2}$ mediated oxidative coupling and $\mathrm{FeCl}_{3}$ promoted Scholl reactions for the synthesis of coronene and perylene derivatives Diarylmethylene derivatives (5-9 and 14-18) or tetraarylmethylene derivatives (29-31) (1.0 mmol), iodine $(0.64 \mathrm{~g}, 2.5 \mathrm{mmol})$ and methyloxyrane $(10 \mathrm{ml})$ were taken in anhydrous toluene $(30 \mathrm{ml})$. The mixture was irradiated under UV-light for $12 \mathrm{~h}$ in nitrogen atmosphere. On completion of reaction, the excessive iodine was removed by treating with saturated sodium thiosulphate solution. Toluene was removed under vacuum, and product mixture (partially and completely annulated products, in case of 5-9 and 14-18 and 34) or pure partially fused products (in case of 29-30) were dried.

Mixture or pure partially fused derivatives (32-33) were dissolved in anhydrous dichloromethane $(60 \mathrm{ml})$ and stirred in nitrogen atmosphere. Iron chloride (20.0-40.0 $\mathrm{mmol})$ as dissolved in nitromethane $(10 \mathrm{ml})$ was added via syringe, and reaction mixture was further stirred at room temperature for 4-36 $\mathrm{h}$ depending on the reactivity of the substrate. On completion of reaction, the reaction mixture was quenched by adding methanol, and the precipitated product was filtered. It was successively washed with methanol, hydrochloric acid and water then dried. Depending on the solubility, it was purified either by recrystallization or by column chromatography.

Preparation of dibenzo $[a, d]$ coronene (DBC) According to general reaction procedure, 6-(diphenylmethylene)-6H-benzo[cd]pyrene (5) (0.40 g, $1.0 \mathrm{mmol})$, iodine and methyloxirane were reacted to furnish a mixture of partially and completely annulated products, then product mixture was treated with iron chloride (3.24 g, $20.0 \mathrm{mmol})$ for 24 h. Product was obtained by re-crystallization with benzene, Yield: $0.30 \mathrm{~g}, 76 \%$. Mp:

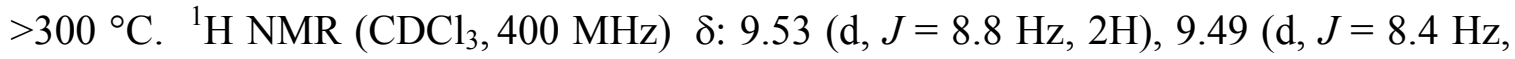
2H), $9.38(\mathrm{~d}, J=8.4 \mathrm{~Hz}, 2 \mathrm{H}), 8.81(\mathrm{~d}, J=8.8 \mathrm{~Hz}, 2 \mathrm{H}), 8.71-8.65(\mathrm{~m}, 4 \mathrm{H}), 8.02(\mathrm{t}, J=7.2$ $\mathrm{Hz}, 2 \mathrm{H}), 7.93(\mathrm{t}, J=7.2 \mathrm{~Hz}, 2 \mathrm{H}) .{ }^{13} \mathrm{C}$ NMR could not brecorded due to poor solubility of the derivative. MALDI-TOF MS: calculated for $\left[\mathrm{C}_{32} \mathrm{H}_{16}\right]^{+}: 400.1246$, found: 400.1249 .

Preparation of 8,13-dimethoxydibenzo $[a, d]$ coronene (Met-DBC) According to general reaction procedure, 6-(bis(4-methoxyphenyl)methylene)-6H-benzo[cd]pyrene (6) (0.46 g, $1.0 \mathrm{mmol}$ ), iodine and methyloxirane were reacted to furnish the mixture of partially and 


\section{Supporting Information}

completely annulated products, then product mixture was treated with iron chloride $(3.24$ g, $20.0 \mathrm{mmol}$ ) for $6 \mathrm{~h}$. Product was purified by column chromatography using dichloromethane as eluent to afford yellow solid. Yield: $0.36 \mathrm{~g}, 80 \%$. Mp: $275{ }^{\circ} \mathrm{C} .{ }^{1} \mathrm{H}$ $\operatorname{NMR}\left(\mathrm{CDCl}_{3}, 400 \mathrm{MHz}\right) \delta: 9.36(\mathrm{~d}, J=8.8 \mathrm{~Hz}, 2 \mathrm{H}), 9.29(\mathrm{~d}, J=9.2 \mathrm{~Hz}, 2 \mathrm{H}), 8.71$ (d, $J$ $=8.8 \mathrm{~Hz}, 2 \mathrm{H}), 8.65-8.62(\mathrm{~m}, 6 \mathrm{H}), 7.54(\mathrm{dd}, J=11.6 \mathrm{~Hz}, 2.4 \mathrm{~Hz}, 2 \mathrm{H}), 4.24(\mathrm{~s}, 6 \mathrm{H}) .{ }^{13} \mathrm{C}$ NMR $\left(\mathrm{CDCl}_{3}, 100 \mathrm{MHz}\right) \delta: 158.2,131.7,131.4,129.8,128.8,126.5,126.1,125.5,124.7$, 123.8, 123.5, 123.2, 123.0, 121.2, 119.8, 115.6, 104.5, 55.7. MALDI-TOF MS: calculated for $\left[\mathrm{C}_{34} \mathrm{H}_{20} \mathrm{O}_{2}\right]^{+}: 460.1463$, found: 460.1457 .

Preparation of 8,13-difluorodibenzo $[a, d]$ coronene (Flu-DBC) According to general reaction procedure, 6-(Bis(4-methoxyphenyl)methylene)-6H-benzo[cd]pyrene (6) (0.44 $\mathrm{g}, 1.0 \mathrm{mmol}$ ), iodine and methyloxirane were reacted to furnish the mixture of partially and completely annulated products, then product mixture was treated with iron chloride (3.24 g, $20.0 \mathrm{mmol}$ ) for $20 \mathrm{~h}$. Product was purified by vacuum sublimation at $420{ }^{\circ} \mathrm{C}$ $\left(5 * 10^{-5}\right.$ torr) to afford yellow solid. Yield: $0.30 \mathrm{~g}, 65 \%$. Mp: $>300{ }^{\circ} \mathrm{C}$. Due to low solubility of the derivative, NMR data could not be obtained. MALDI-TOF MS: calculated for $\left[\mathrm{C}_{32} \mathrm{H}_{14} \mathrm{~F}_{2}\right]^{+}: 436.1058$, found: 436.1047 .

Preparation of dinaphtho[1,2-a:2', $\left.1^{\prime}-d\right]$ coronene (DNC) According to general reaction procedure, 6-(Di(naphthalen-2-yl)methylene)-6H-benzo[cd]pyrene (8) (0.50 g, 1.0 mmol), iodine and methyloxirane were reacted to furnish the mixture of partially and completely annulated products, then product mixture was treated with iron chloride (3.24 g, $20.0 \mathrm{mmol}$ ) for $12 \mathrm{~h}$. Product was obtained by re-crystallization with benzene to give light yellow solid. Yield: $0.39 \mathrm{~g}, 77 \%$. Mp: $>300{ }^{\circ} \mathrm{C} .{ }^{1} \mathrm{H}$ NMR $\left(\mathrm{CDCl}_{3}, 400 \mathrm{MHz}\right) \delta$ : $10.04(\mathrm{~d}, J=8.8 \mathrm{~Hz}, 2 \mathrm{H}), 9.50(\mathrm{~d}, J=8.4 \mathrm{~Hz}, 2 \mathrm{H}), 9.18$ (d, $J=8.8 \mathrm{~Hz}, 2 \mathrm{H}), 8.91$ (d, $J=$ $9.2 \mathrm{~Hz}, 2 \mathrm{H}), 8.84(\mathrm{~s}, 4 \mathrm{H}), 8.26(\mathrm{~d}, J=7.2 \mathrm{~Hz}, 2 \mathrm{H}), 8.12$ (d, $J=8.8 \mathrm{~Hz}, 2 \mathrm{H}), 7.92-7.82$ $(\mathrm{m}, 4 \mathrm{H}) .{ }^{13} \mathrm{C}$ NMR could not be recorded due to poor solubility of the derivative. MALDI-TOF MS: calculated for $\left[\mathrm{C}_{40} \mathrm{H}_{20}\right]^{+}: 500.1559$, found: 500.1552 .

Preparation of coroneno[1,2-b:4,3-b']dithiophene (DTC) According to general reaction procedure, 3,3'-((6H-benzo[ $c d]$ pyren-6-ylidene)methylene)dithiophene (9) $(0.42$ $\mathrm{g}, 1.0 \mathrm{mmol}$ ), iodine and methyloxirane were reacted to furnish the mixture of partially and completely annulated products, then product mixture was treated with iron chloride 


\section{Supporting Information}

(3.24 g, $20.0 \mathrm{mmol}$ ) for $12 \mathrm{~h}$. A mixture of product and by-products was obtained that could not be purified.

Preparation of benzo[b]naphtho[1,2,3,4-pqr]perylene (BNP) According to general reaction procedure, 7-(diphenylmethylene)-7H-benzo[de]anthracene (14) (0.38 g, 1.0 $\mathrm{mmol}$ ), iodine and methyloxirane were reacted to furnish the mixture of partially and completely annulated products, then product mixture was treated with iron chloride $(3.24$ $\mathrm{g}, 20.0 \mathrm{mmol}$ ) for $24 \mathrm{~h}$. Product was obtained by re-crystallization from benzene to afford light-yellow solid. Yield: 0.26 g, 70\%. Mp: $295{ }^{\circ} \mathrm{C} .{ }^{1} \mathrm{H} \mathrm{NMR}\left(\mathrm{CDCl}_{3}, 400 \mathrm{MHz}\right)$ 8: 9.15 $(\mathrm{d}, J=7.2 \mathrm{~Hz}, 1 \mathrm{H}), 9.05$ (d, $J=9.2 \mathrm{~Hz}, 1 \mathrm{H}), 8.77-8.70(\mathrm{~m}, 4 \mathrm{H}), 8.63$ (d, $J=8.4 \mathrm{~Hz}, 1 \mathrm{H})$, $8.59(\mathrm{~d}, J=8.0 \mathrm{~Hz}, 1 \mathrm{H}), 8.20$ (d, $J=7.2 \mathrm{~Hz}, 1 \mathrm{H}), 8.06$ (s, 1H), 8.04 (s, 1H), 7.96-7.92 $(\mathrm{m}, 1 \mathrm{H}), 7.78-7.74(\mathrm{~m}, 1 \mathrm{H}), 7.64-7.61(\mathrm{~m}, 1 \mathrm{H}), 7.45-7.35(\mathrm{~m}, 2 \mathrm{H}) .{ }^{13} \mathrm{C}$ NMR could not be recorded due to poor solubility of the derivative. MALDI-TOF MS: calculated for $\left[\mathrm{C}_{30} \mathrm{H}_{16}\right]^{+}:$376.1246, found: 376.1241 .

\section{Preparation of 2,7-dimethoxybenzo[b]naphtho[1,2,3,4-pqr]perylene (Met-BNP)}

According to general reaction procedure, 7-(bis(4-methoxyphenyl)methylene)-7Hbenzo[de]anthracene (15) $(0.44 \mathrm{~g}, 1.0 \mathrm{mmol})$, iodine and methyloxirane were reacted to furnish the mixture of partially and completely annulated products, then product mixture was treated with iron chloride $(3.24 \mathrm{~g}, 20.0 \mathrm{mmol})$ for $6 \mathrm{~h}$. Product was purified by column chromatography using dichloromethane as eluent to afford yellow solid. Yield: 0.37 g, 85\%. Mp: $211^{\circ} \mathrm{C} .{ }^{1} \mathrm{H}$ NMR (DMSO-D, $\left.400 \mathrm{MHz}\right) \delta: 9.17$ (d, $J=9.2 \mathrm{~Hz}, 1 \mathrm{H}$ ), 9.09-9.06 (m, 2H), 9.01 (d, $J=7.6 \mathrm{~Hz}, 1 \mathrm{H}), 8.97$ (d, $J=9.2 \mathrm{~Hz}, 1 \mathrm{H}), 8.75$ (d, $J=8.8 \mathrm{~Hz}$, $1 \mathrm{H}), 8.57(\mathrm{~d}, J=2.0 \mathrm{~Hz}, 1 \mathrm{H}), 8.39-8.30(\mathrm{~m}, 3 \mathrm{H}), 8.08-8.02(\mathrm{~m}, 2 \mathrm{H}), 7.53(\mathrm{dd}, J=11.6$ $\mathrm{Hz} 2.4 \mathrm{~Hz}, 1 \mathrm{H}), 7.41(\mathrm{dd}, J=11.6 \mathrm{~Hz} 2.4 \mathrm{~Hz}, 1 \mathrm{H}), 4.16(\mathrm{~s}, 3 \mathrm{H}), 4.10(\mathrm{~s}, 3 \mathrm{H}) .{ }^{13} \mathrm{C} \mathrm{NMR}$ (DMSO-D, $100 \mathrm{MHz}$ ) $\delta: 158.9,158.3,132.8,132.5,131.6,131.3,130.5,130.2,129.9$, $129.3,129.2$, 127.6, 127.2, 125.3, 125.2, 125.1, 124.8, 123.4, 123.0, 122.7, 122.1, 121.4, 119.7, 117.1, 116.1, 106.5, 104.2, 56.0. MALDI-TOF MS: calculated for $\left[\mathrm{C}_{32} \mathrm{H}_{20} \mathrm{O}_{2}\right]^{+}$: 436.1457, found: 436.1455 .

\section{Preparation of 2,7-difluorobenzo[b]naphtho[1,2,3,4-pqr]perylene (Flu-BNP)} According to general reaction procedure, 7-(bis(4-fluorophenyl)methylene)-7Hbenzo[de]anthracene (16) $(0.41 \mathrm{~g}, 1.0 \mathrm{mmol})$, iodine and methyloxirane were reacted to furnish a mixture of partially and completely annulated products, then product mixture 


\section{Supporting Information}

was treated with iron chloride $(3.24 \mathrm{~g}, 20.0 \mathrm{mmol})$ for $24 \mathrm{~h}$. Product was obtained by recrystallization from benzene to afford a light-yellow solid. Yield: $0.28 \mathrm{~g}, 69 \%$. Mp: >300 ${ }^{\circ} \mathrm{C} .{ }^{1} \mathrm{H}$ NMR (DMSO-D, $\left.400 \mathrm{MHz}\right) \delta: 9.34$ (d, $\left.J=10.0 \mathrm{~Hz}, 1 \mathrm{H}\right), 9.02$ (d, $J=9.6 \mathrm{~Hz}$, $1 \mathrm{H}), 8.91-8.86(\mathrm{~m}, 2 \mathrm{H}), 8.69$ (d, $J=8.4 \mathrm{~Hz}, 1 \mathrm{H}), 7.95-7.91(\mathrm{~m}, 2 \mathrm{H}), 7.68$ (t, $J=8.8 \mathrm{~Hz}$, $1 \mathrm{H}), 7.63-7.48(\mathrm{~m}, 5 \mathrm{H}), 7.15(\mathrm{t}, J=7.6 \mathrm{~Hz}, 1 \mathrm{H}) .{ }^{13} \mathrm{C}$ NMR could not recorded due to poor solubility of the derivative. MALDI-TOF MS: calculated for $\left[\mathrm{C}_{30} \mathrm{H}_{14} \mathrm{~F}_{2}\right]_{2}{ }^{+}: 824.2128$, found: 824.2302 .

Preparation of naphtho[2,1-b]phenanthro[4,3,2,1-pqr]perylene (NPP) According to general reaction procedure, 7-(di(naphthalen-2-yl)methylene)-7H-benzo[de]anthracene (17) $(0.48 \mathrm{~g}, 1.0 \mathrm{mmol})$, iodine and methyloxirane were reacted to furnish a mixture of partially and completely annulated products, then product mixture was treated with iron chloride $(3.24 \mathrm{~g}, 20.0 \mathrm{mmol})$ for $12 \mathrm{~h}$. Product was obtained by re-crystallization in benzene to afford a light yellow solid. Yield: $0.34 \mathrm{~g}, 71 \% \mathrm{Mp}: 253{ }^{\circ} \mathrm{C} .{ }^{1} \mathrm{H} \mathrm{NMR}\left(\mathrm{CDCl}_{3}\right.$, $400 \mathrm{MHz}) \delta: 9.38(\mathrm{~d}, J=9.2 \mathrm{~Hz}, 1 \mathrm{H}), 9.32$ (d, $J=8.0 \mathrm{~Hz}, 1 \mathrm{H}), 9.21$ (t, $J=8.0 \mathrm{~Hz}, 2 \mathrm{H})$, $9.03(\mathrm{t}, J=7.2 \mathrm{~Hz}, 2 \mathrm{H}), 8.88(\mathrm{~d}, J=9.2,1 \mathrm{H}), 8.80(\mathrm{~d}, J=8.8,1 \mathrm{H}), 8.25(\mathrm{~d}, J=8.8,2 \mathrm{H})$, 8.17-7.96 (m, 6H), 7.82-7.68 (m, 4H). ${ }^{13} \mathrm{C}$ NMR could not recorded due to poor solubility of the derivative. MALDI-TOF MS: calculated for $\left[\mathrm{C}_{38} \mathrm{H}_{20}\right]^{+}: 476.1559$, found: 476.1570 . Preparation of 1,6,8-triphenyldibenzo[f,pqr]tetraphene (32) According to general reaction procedure, 7-(diphenylmethylene)-3,9-diphenyl-7H-benzo[de]anthracene (29) $(0.53 \mathrm{~g}, 1.0 \mathrm{mmol})$, iodine and methyloxirane were reacted to furnish the partially annulated product as light-yellow solid. Yield: $0.41 \mathrm{~g}, 78 \%$. Mp: $245{ }^{\circ} \mathrm{C} .{ }^{1} \mathrm{H}$ NMR $\left(\mathrm{CDCl}_{3}, 400 \mathrm{MHz}\right) \delta: 9.00(\mathrm{t}, J=7.6 \mathrm{~Hz}, 2 \mathrm{H}), 8.80$ (d, $\left.J=8.0 \mathrm{~Hz}, 1 \mathrm{H}\right), 8.69$ (d, $J=8.4$ $\mathrm{Hz}, 1 \mathrm{H}), 8.36$ (d, $J=9.6 \mathrm{~Hz}, 1 \mathrm{H}), 8.30$ (s, 1H), 7.95-7.92 (m, 2H), 7.80-7.53 (m, 13H), 7.37-7.31 (m, 3H), 7.14-7.11 (m, 2H). ${ }^{13} \mathrm{C} \mathrm{NMR}\left(\mathrm{CDCl}_{3}, 100 \mathrm{MHz}\right) \delta: 144.5,143.8$, 143.5. 140.7, 140.2, 138.5, 134.5, 133.7, 132.7, 132.5, 131.1, 130.4, 130.2, 129.8, 129.7, $129.5,128.9,128.7,128.5,128.4,128.3,127.8,127.3,127.1,127.0,126.4,126.3,125.4$, 124.9, 124.1, 119.4. MALDI-TOF MS: calculated for $\left[\mathrm{C}_{42} \mathrm{H}_{26}\right]^{+}$: 530.2029, found: 530.2029 .

\section{Preparation of 11-methoxy-8-(4-methoxyphenyl)-1,6-diphenyldibenzo[f,pqr]tetra- -}

phene (33) According to general reaction procedure, 7-(bis(4methoxyphenyl)methylene)-3,9-diphenyl-7H-benzo[de]anthracene $\quad(30) \quad(0.59$ g, 1.0 


\section{Supporting Information}

mmol), iodine and methyloxirane were reacted to furnish the partially annulated product as light-yellow solid. Yield: $0.45 \mathrm{~g}, 76 \%$. Mp: $300{ }^{\circ} \mathrm{C} .{ }^{1} \mathrm{H} \mathrm{NMR}\left(\mathrm{CDCl}_{3}, 400 \mathrm{MHz}\right) \delta$ : $8.87(\mathrm{~d}, J=8.8 \mathrm{~Hz}, 1 \mathrm{H}), 8.79$ (d, $J=8.4 \mathrm{~Hz}, 1 \mathrm{H}), 8.68$ (d, $J=7.6 \mathrm{~Hz}, 1 \mathrm{H}), 8.33-8.28$ (m, 2H), $8.21(\mathrm{~d}, J=2.0 \mathrm{~Hz}, 1 \mathrm{H}), 7.95-7.92(\mathrm{~m}, 2 \mathrm{H}), 7.72(\mathrm{dd}, J=13.0 \mathrm{~Hz}, 2.0 \mathrm{~Hz}, 1 \mathrm{H})$, 7.69-7.52 (m, 7H), 7.39-7.33 (m, 4H), 7.22-7.19 (m, 4H), 4.09 (s, 3H), $3.99(\mathrm{~s}, 3 \mathrm{H}) .{ }^{13} \mathrm{C}$ NMR $\left(\mathrm{CDCl}_{3}, 100 \mathrm{MHz}\right) \delta: 159.3,158.0,141.2,140.8,138.9,138.0,135.8,135.3,132.7$, $131.7,131.0,130.5,130.2,129.6,129.5,128.4,127.6,127.5,127.3,127.1,125.9,129.7$, 125.4, 124.7, 123.9, 121.9, 119.4, 117.8, 115.1, 102.5, 55.5. MALDI-TOF MS: calculated for $\left[\mathrm{C}_{44} \mathrm{H}_{30} \mathrm{O}_{2}\right]^{+}: 590.2240$, found: 590.2226 .

Preparation of 18-phenylbenzo[b]indeno[1,2,3- $\mathrm{lm}]$ naphtho[1,2,3,4-pqr]perylene $(\mathrm{Ph}$ BINP) According to general reaction procedure, 1,6,8-triphenyldibenzo[f,pqr]tetraphene (32) $(0.53 \mathrm{~g}, 1.0 \mathrm{mmol})$ and iron chloride $(4.86 \mathrm{~g}, 30.0 \mathrm{mmol})$ were reacted for $36 \mathrm{~h}$. The product was purified by column chromatography using $\mathrm{dcm} / \mathrm{hexane}$ eluent to afford a yellow-orange solid. Yield: $0.42 \mathrm{~g}, 80 \%$. Mp: $>300{ }^{\circ} \mathrm{C} .{ }^{1} \mathrm{H} \mathrm{NMR}\left(\mathrm{CDCl}_{3}, 400 \mathrm{MHz}\right) \delta$ : $9.18(\mathrm{~d}, J=8.4 \mathrm{~Hz}, 1 \mathrm{H}), 9.08(\mathrm{~s}, J=9.6 \mathrm{~Hz}, 1 \mathrm{H}), 9.00(\mathrm{~d}, J=8.4 \mathrm{~Hz}, 1 \mathrm{H}), 8.80$ (d, $J=$ $8.0 \mathrm{~Hz}, 1 \mathrm{H}), 8.49$ (d, $J=8.4 \mathrm{~Hz}, 1 \mathrm{H}), 8.04-7.75(\mathrm{~m}, 7 \mathrm{H}), 7.64-7.45(\mathrm{~m}, 8 \mathrm{H}), 7.19$ (t, $J=$ $8.0 \mathrm{~Hz}, 1 \mathrm{H}) .{ }^{13} \mathrm{C} \mathrm{NMR}\left(\mathrm{CDCl}_{3}, 100 \mathrm{MHz}\right) \delta: 144.7,138.7,135.1,134.3,131.6,131.0$, $130.9,130.8,130.5,130.3,129.8,129.6,129.5,129.4,129.2,128.0,127.8,127.2,127.0$, $126.4,126.2$, 125.4, 125.0, 123.2, 122.1, 121.9, 121.7, 121.6, 120.8, 120.1, 115.9. MALDI-TOF MS: calculated for $\left[\mathrm{C}_{42} \mathrm{H}_{22}\right]^{+}:$526.1716, found: 526.1717 .

Preparation of 2,7-dimethoxy-9,14-diphenylbenzo[b]naphtho[1,2,3,4-pqr]perylene (Met-PhBNP) According to general reaction procedure, 11-methoxy-8-(4methoxyphenyl)-1,6-diphenyldibenzo[f,pqr]tetraphene (33) (0.59 g, $1.0 \mathrm{mmol})$ and iron chloride $(3.24 \mathrm{~g}, 20.0 \mathrm{mmol})$ were reacted for $4 \mathrm{~h}$. The product was purified by column chromatography using $\mathrm{dcm} /$ hexane as eluent to afford an orange solid. Yield: $0.48 \mathrm{~g}$, 81\%. Mp: $295^{\circ} \mathrm{C} .{ }^{1} \mathrm{H}$ NMR $\left(\mathrm{CDCl}_{3}, 400 \mathrm{MHz}\right) \delta: 9.02$ (d, J=9.2 Hz, 1H), 8.78-8.75 (m, $3 \mathrm{H}), 8.56(\mathrm{~d}, J=9.2 \mathrm{~Hz}, 1 \mathrm{H}), 8.30(\mathrm{~d}, J=2.8 \mathrm{~Hz}, 1 \mathrm{H}), 8.26(\mathrm{~d}, J=9.6 \mathrm{~Hz}, 1 \mathrm{H}), 7.88-$ $7.84(\mathrm{~m}, 2 \mathrm{H}), 7.71-7.42(\mathrm{~m}, 12 \mathrm{H}), 7.05(\mathrm{dd}, J=11.6 \mathrm{~Hz} 2.8 \mathrm{~Hz}, 1 \mathrm{H}), 4.13(\mathrm{~s}, 3 \mathrm{H}), 3.33$ (s, 3H). ${ }^{13} \mathrm{C} \mathrm{NMR}\left(\mathrm{CDCl}_{3}, 100 \mathrm{MHz}\right) \delta: 156.4,145.0,141.1,138.8,138.2,132.3,131.3$, $131.1,130.7,130.4,130.1,130.0,129.8,129.6,129.2$, 128.9, 128.4, 127.8, 127.6, 127.3, $127.1,126.7,125.8,125.2,125.0,124.7,123.2$, 122.3, 122.0, 121.6, 120.6, 120.2 , 116.2, 
111.4, 103.6, 55.5. MALDI-TOF MS: calculated for $\left[\mathrm{C}_{44} \mathrm{H}_{28} \mathrm{O}_{2}\right]^{+}: 588.2083$, found: 588.2099 .

\section{Preparation of 5,10-dimethyltribenzo $[a, g h i, k]$ fluorantheno[2,3,4-cde $]$ perylene (Met-}

BFP) According to general reaction procedure, 11-Methoxy-8-(4-methoxyphenyl)-1,6diphenyldibenzo[f,pqr]tetraphene (33) $(0.59 \mathrm{~g}, 1.0 \mathrm{mmol})$ and iron chloride $(4.86 \mathrm{~g}, 30.0$ mmol) were reacted for $12 \mathrm{~h}$. Product was purified by column chromatography using $\mathrm{dcm} /$ hexane eluent to afford an orange solid. Yield: $0.48 \mathrm{~g}, 82 \%$. Mp: $180{ }^{\circ} \mathrm{C} .{ }^{1} \mathrm{H}$ NMR $\left(\mathrm{CDCl}_{3}, 400 \mathrm{MHz}\right)$ 8: 9.95 (s, 1H), $9.71(\mathrm{~d}, J=8.0 \mathrm{~Hz}, 1 \mathrm{H}), 9.44$ (s, 1H), 9.31 (s, 1H), 9.18-9.08 (m, 4H), $8.85(\mathrm{~s}, 1 \mathrm{H}), 8.63(\mathrm{~d}, J=7.2,1 \mathrm{H}), 8.55(\mathrm{~d}, J=6.4,1 \mathrm{H}), 8.29(\mathrm{~d}, J=$ 6.8, 1H), 7.97 (d, $J=8.4,1 \mathrm{H}), 7.88-7.81(\mathrm{~m}, 2 \mathrm{H}), 7.63-7.59(\mathrm{~m}, 3 \mathrm{H}), 4.34(\mathrm{~s}, 3 \mathrm{H}), 4.26$ (s, 3H). ${ }^{13} \mathrm{C}$ NMR $\left(\mathrm{CDCl}_{3}, 100 \mathrm{MHz}\right) \delta: 157.3,132.2,130.4,129.8,129.4,128.9,128.4$, 127.1, 126.3, 123.4, 121.8, 121.3, 119.7, 115.2, 102.8, 55.9, 55.3. MALDI-TOF MS: calculated for $\left[\mathrm{C}_{44} \mathrm{H}_{24} \mathrm{O}_{2}\right]^{+}: 584.1770$, found: 584.1777 .

Preparation of 5,10-dimethoxydibenzo $[a, g h i]$ benzo $[8,9]$ fluorantheno[2,3,4$c d e]$ naphtho[2,1-k]perylene (Met-BBFNP) According to general reaction procedure, 7(bis(4-methoxyphenyl)methylene)-3,9-di(naphthalen-2-yl)-7H-benzo[de]anthracene (31) $(0.69 \mathrm{~g}, 1.0 \mathrm{mmol})$, iodine and methyloxirane were reacted to furnish the partially annulated product (34) along with other fused derivatives, then product mixture was treated with iron chloride $(4.86 \mathrm{~g}, 30.0 \mathrm{mmol})$ for $24 \mathrm{~h}$. Product was found to be weakly soluble to characterize by NMR spectroscopy, therefore it could only characterize by mass spectroscopy. Fab HRMS: calculated for $\left[\mathrm{C}_{52} \mathrm{H}_{28} \mathrm{O}_{2}\right]^{+}: 684.2089$, found: 684.2097. 


\section{Supporting Information}

\section{NMR spectra of the derivatives}

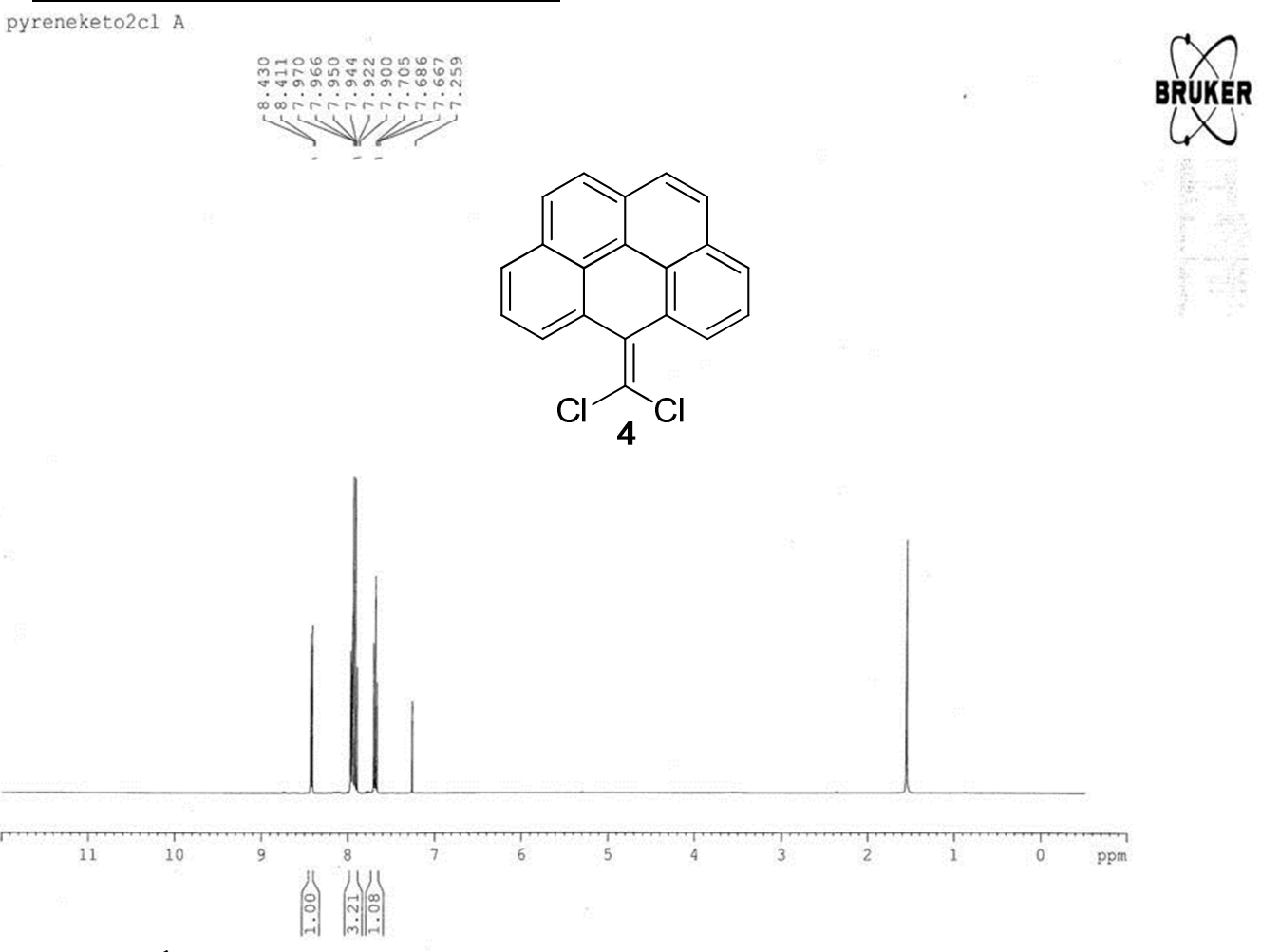

Figure S1. ${ }^{1} \mathrm{H}$ NMR spectrum of 6-(dichlorolmethylene)-6H-benzo[cd]pyrene (4) pyreneketo2cl Ac13
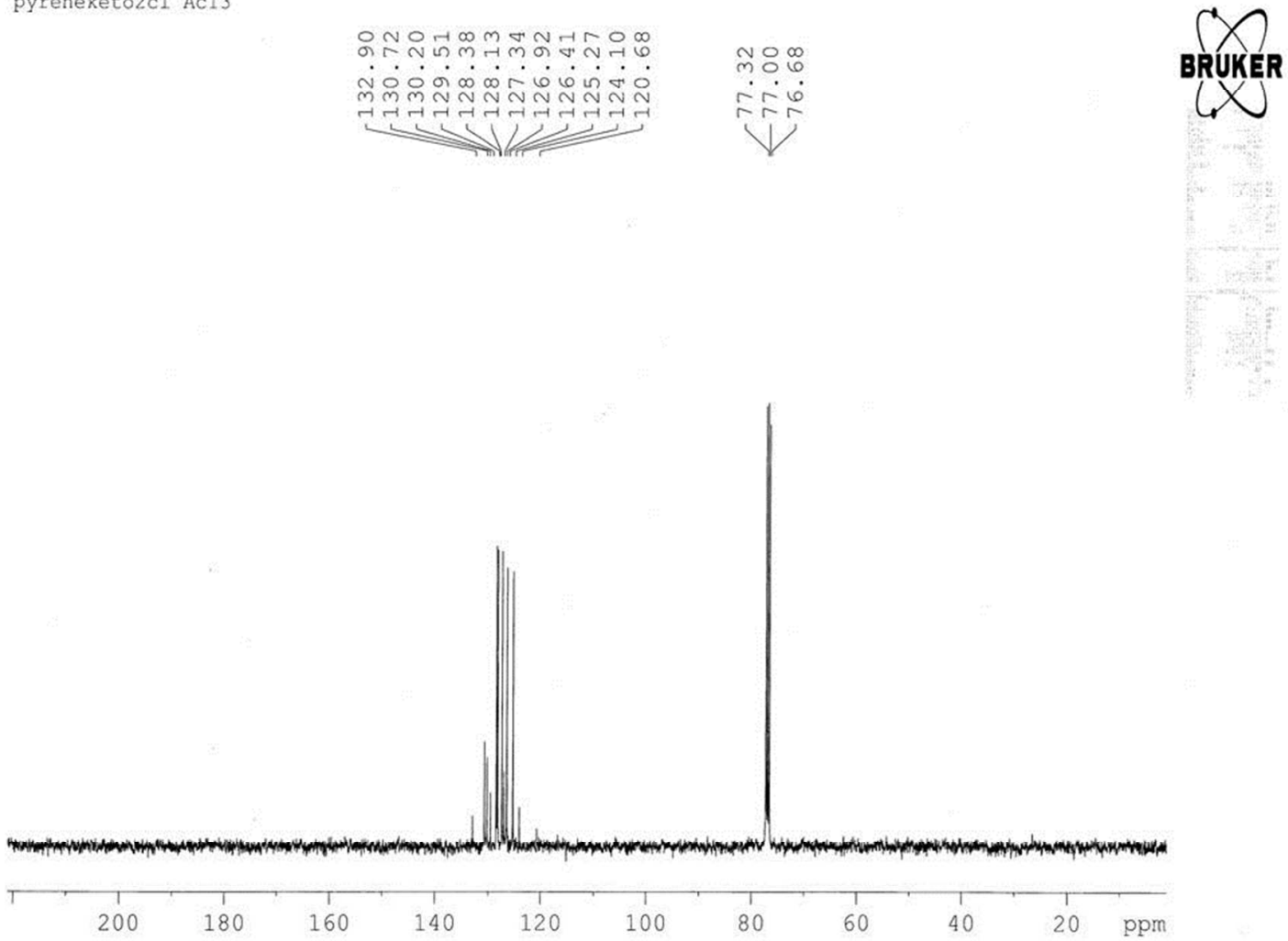

Figure S2. ${ }^{13} \mathrm{C}$ NMR spectrum of 6-(dichlorolmethylene)-6H-benzo[ $\left.c d\right]$ pyrene (4) 
Supporting Information

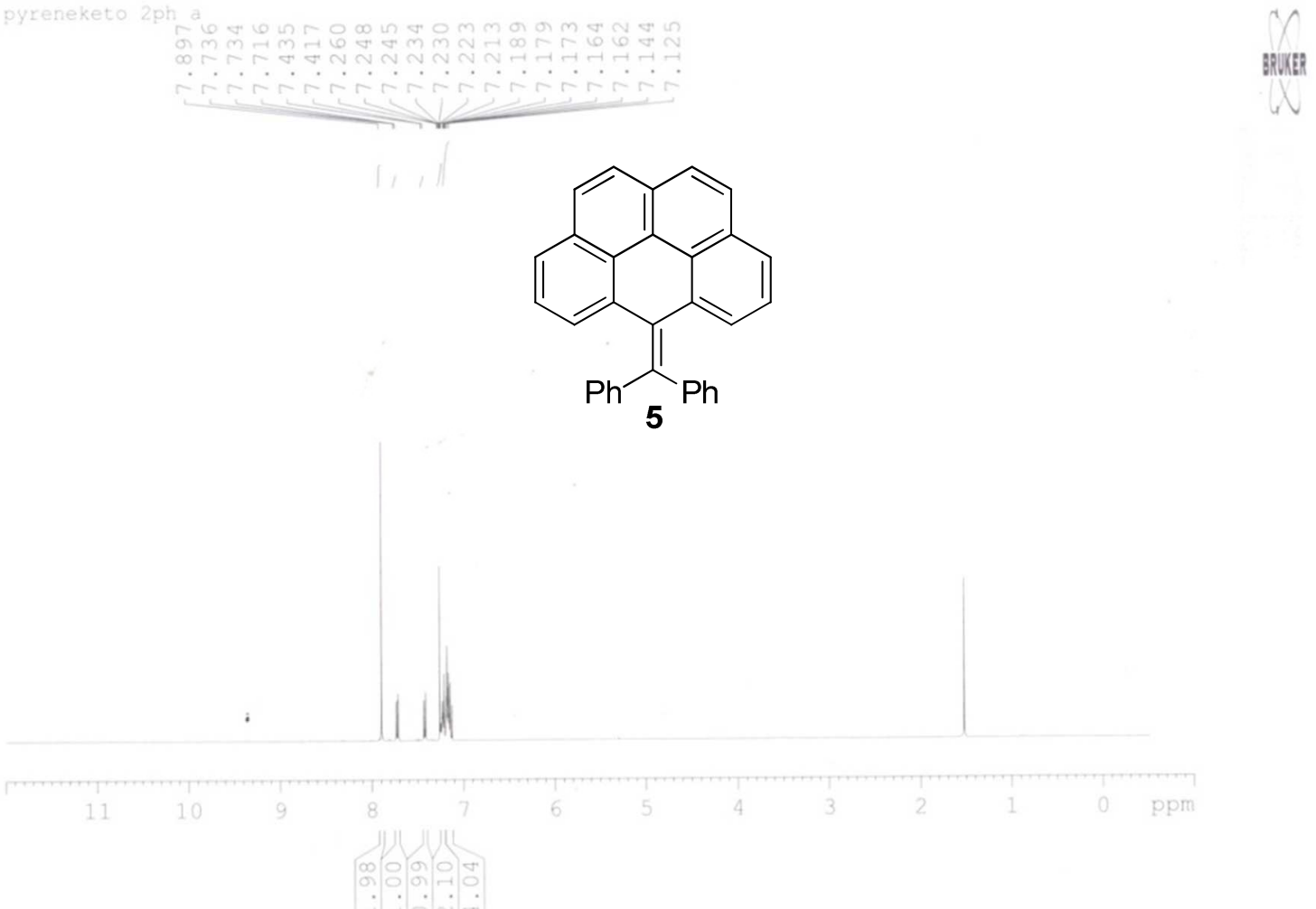

Figure S3. ${ }^{1} \mathrm{H}$ NMR spectrum of 6-(diphenylmethylene)-6H-benzo[cd]pyrene (5)
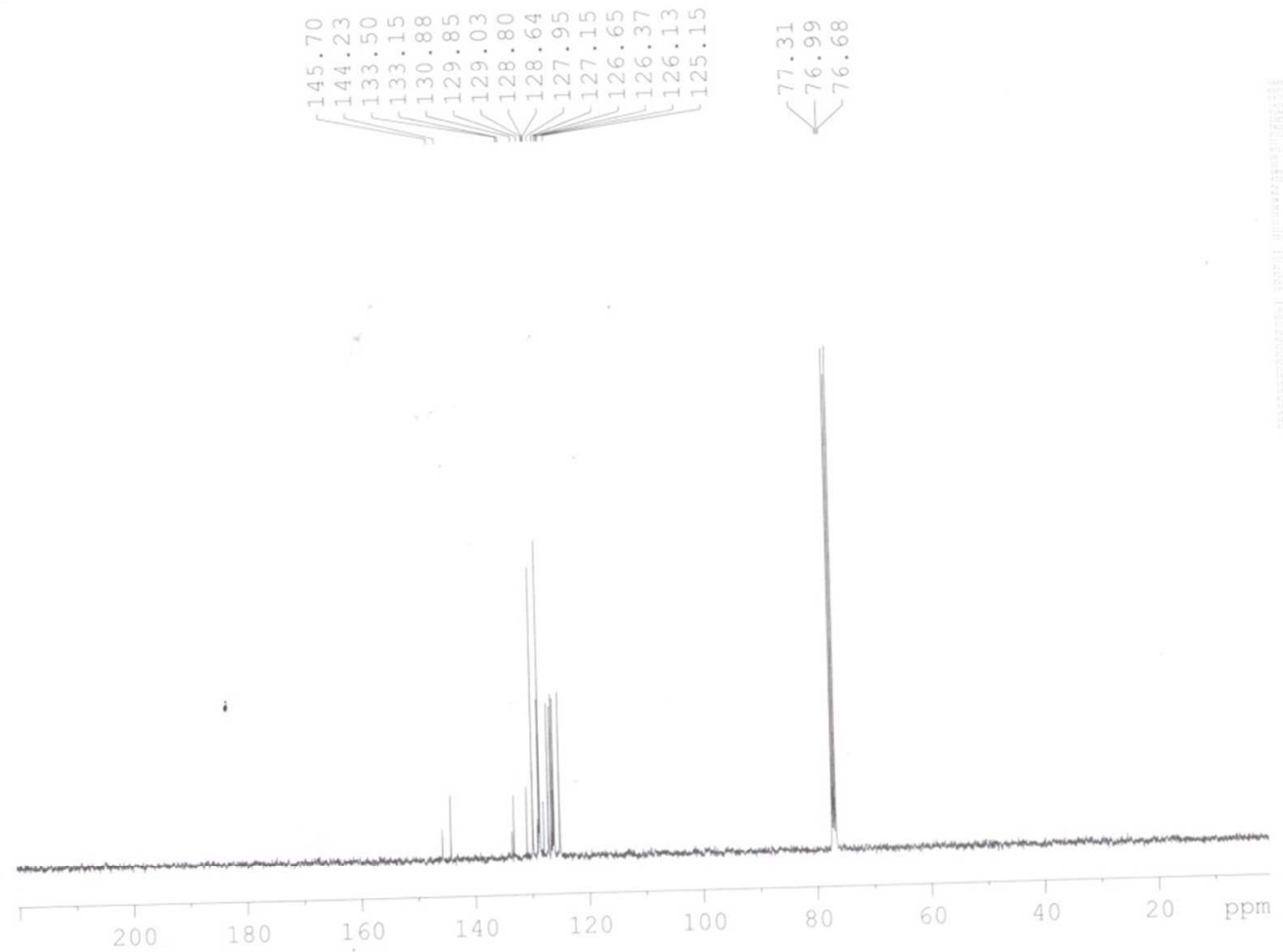

Figure S4. ${ }^{13} \mathrm{C}$ NMR spectrum of 6-(diphenylmethylene)-6H-benzo[cd]pyrene (5) 
Supporting Information

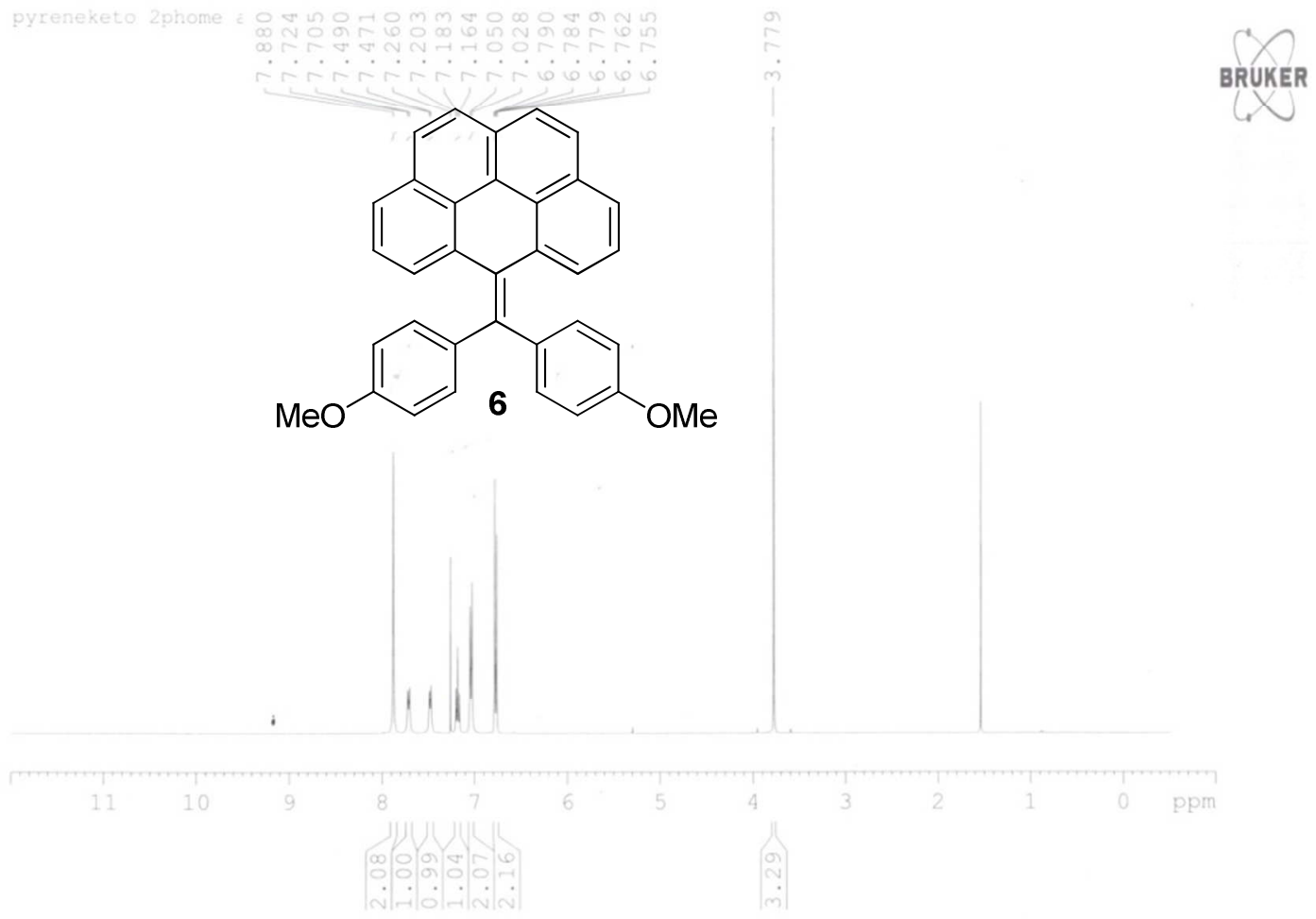

Figure S5. ${ }^{1} \mathrm{H}$ NMR spectrum of 6-(bis(4-methoxyphenyl)methylene)-6Hbenzo $[c d]$ pyrene (6)

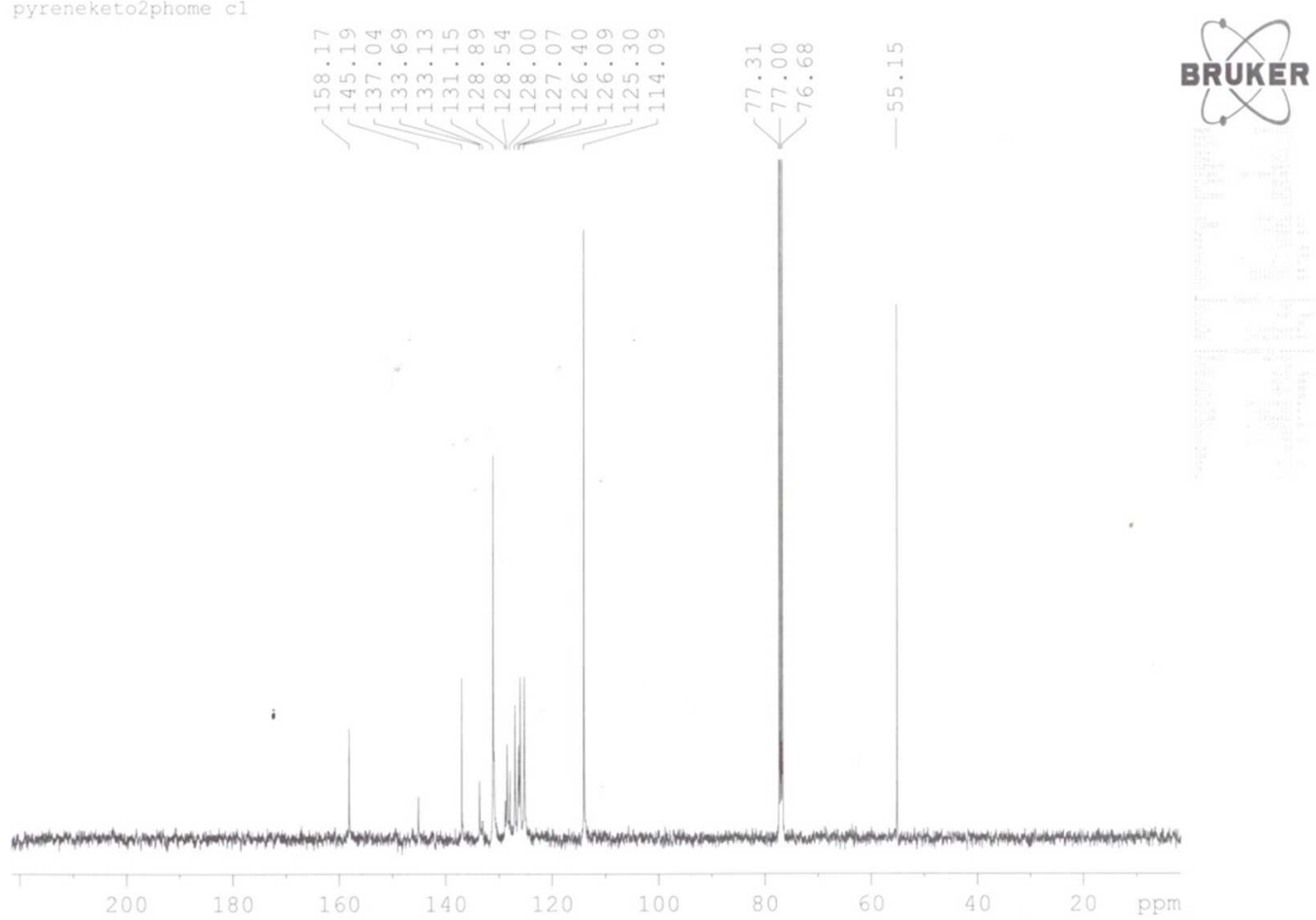

Figure S6. ${ }^{13} \mathrm{C}$ NMR spectrum of 6-(bis(4-methoxyphenyl)methylene)-6Hbenzo $[c d]$ pyrene (6) 
Supporting Information

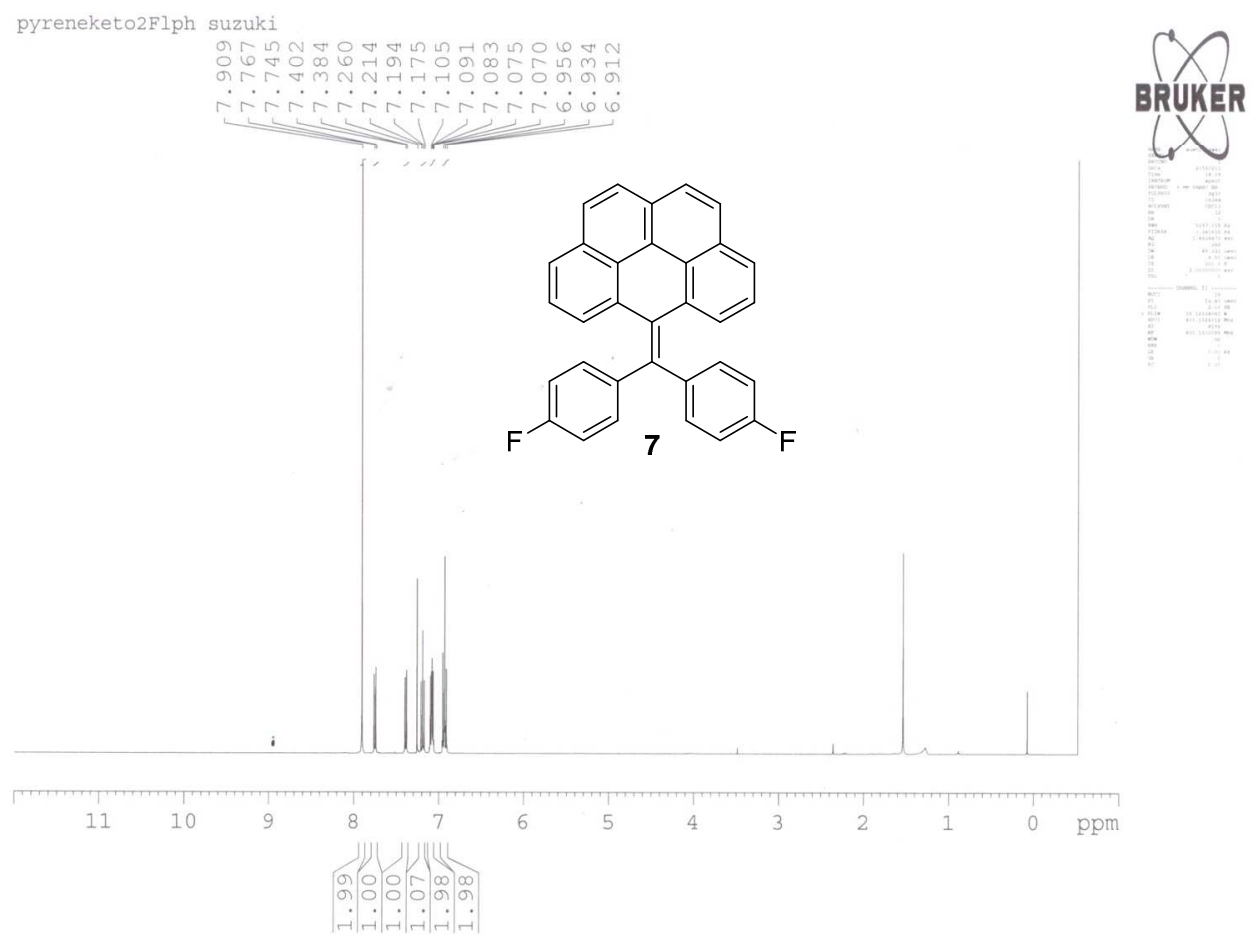

Figure S7. ${ }^{1} \mathrm{H}$ NMR spectrum of 6-(bis(4-fluorophenyl)methylene)-6H-benzo $[c d]$ pyrene (7)

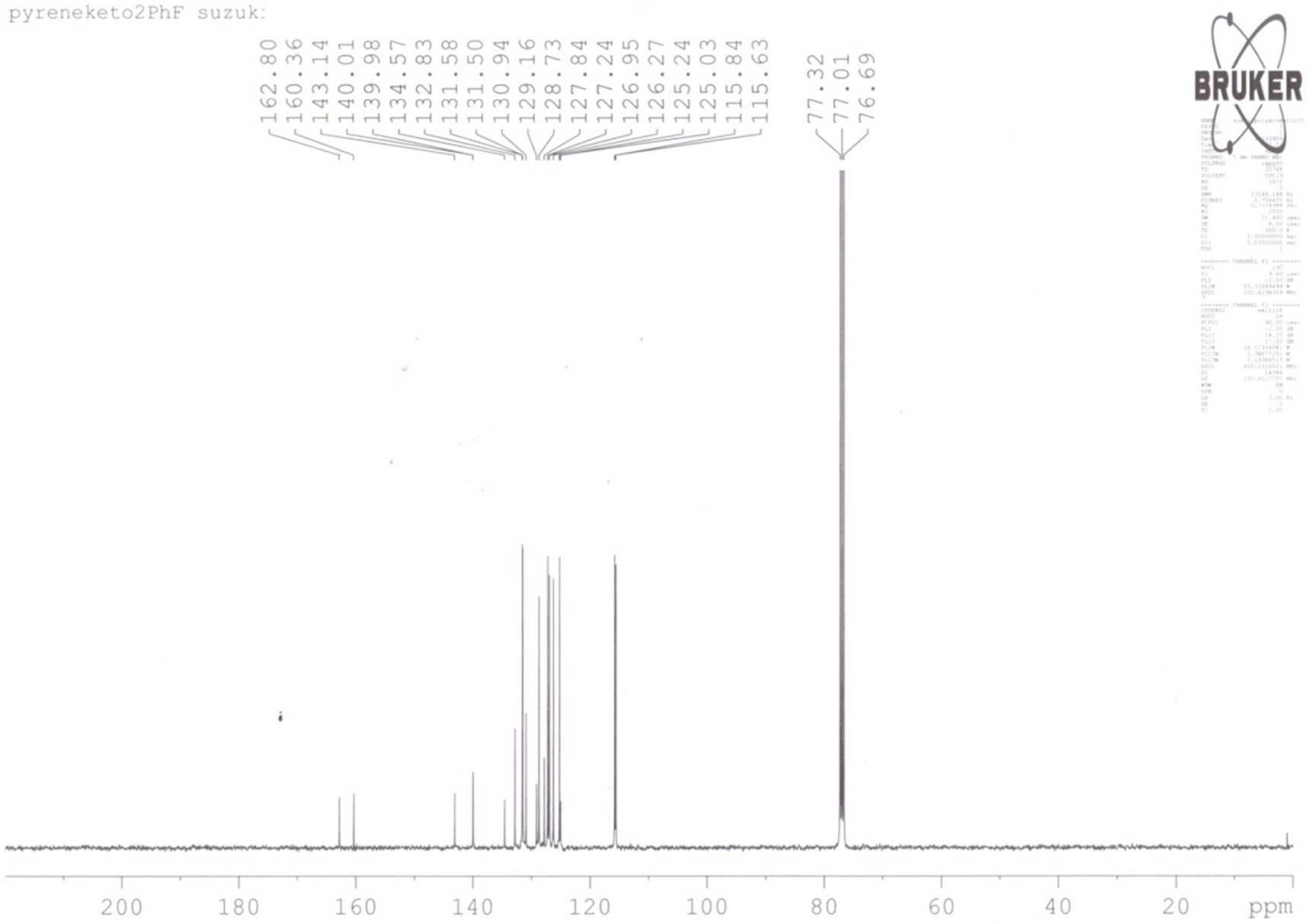

Figure S8. ${ }^{13} \mathrm{C}$ NMR spectrum of 6-(bis(4-fluorophenyl)methylene)-6H-benzo $[c d]$ pyrene (7) 
Supporting Information

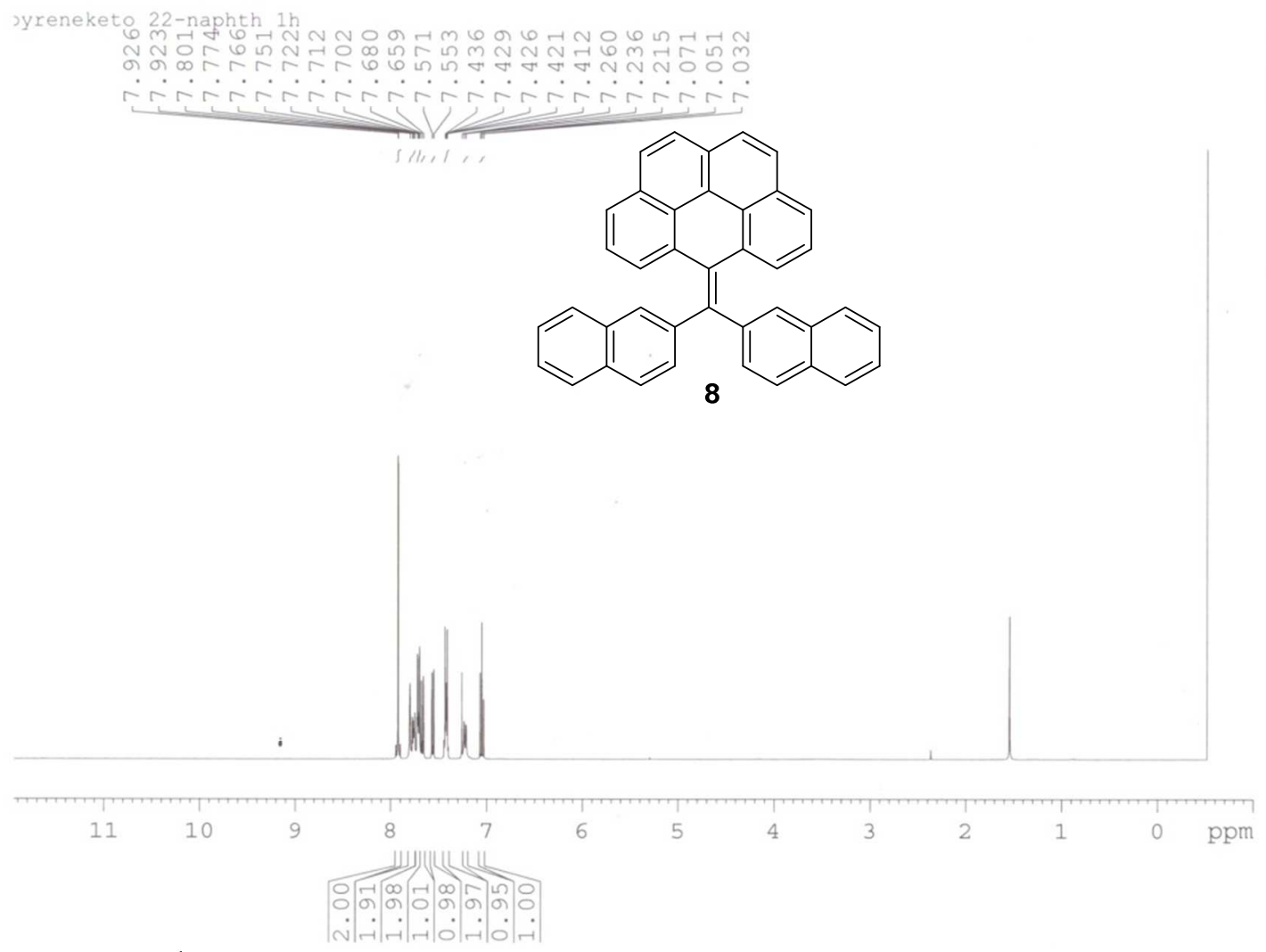

Figure S9. ${ }^{1} \mathrm{H}$ NMR spectrum of 6-(di(naphthalen-2-yl)methylene)-6H-benzo $[c d]$ pyrene
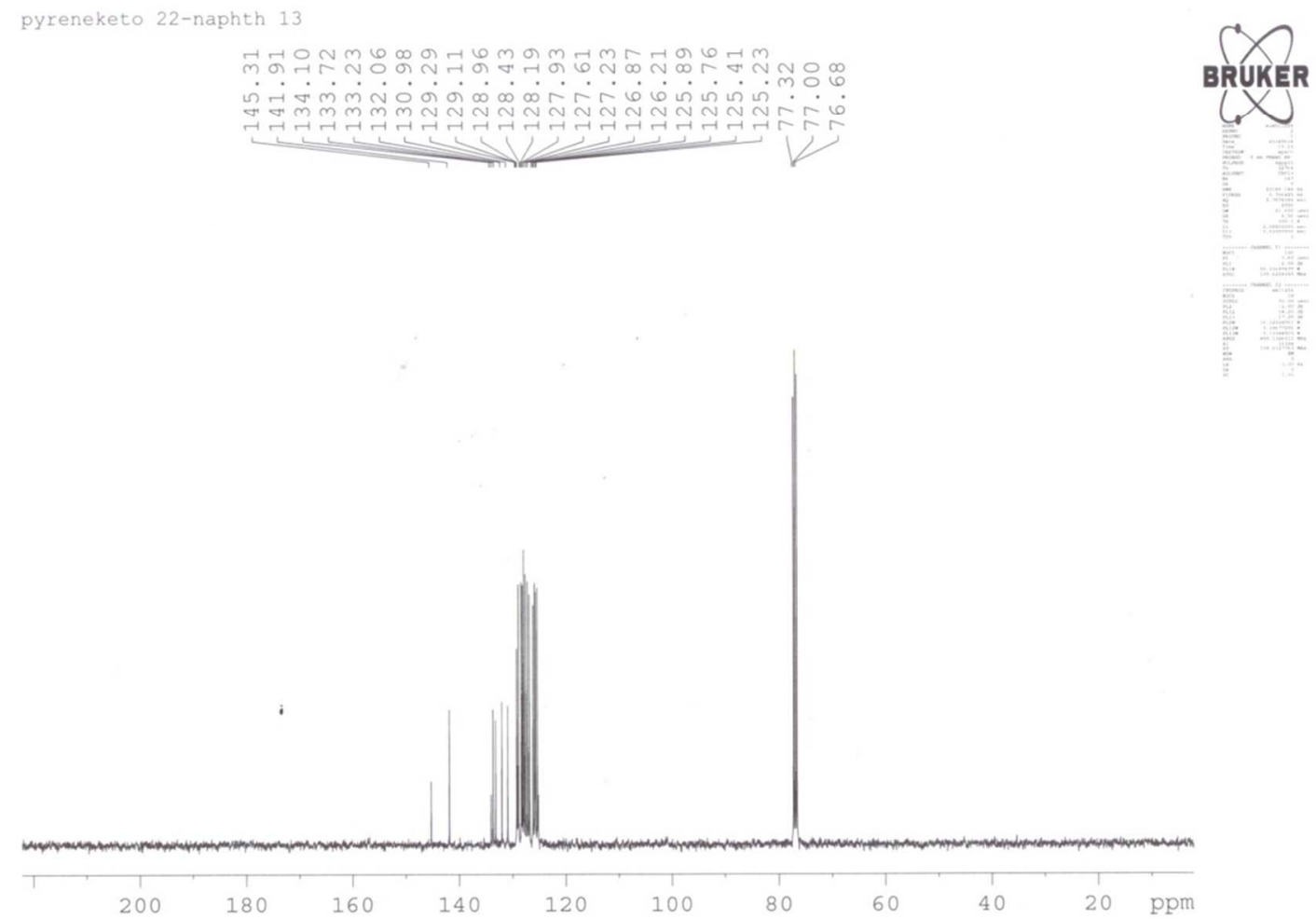

Figure S10. ${ }^{13} \mathrm{C}$ NMR spectrum of 6-(di(naphthalen-2-yl)methylene)-6Hbenzo $[c d]$ pyrene $(\mathbf{8})$ 


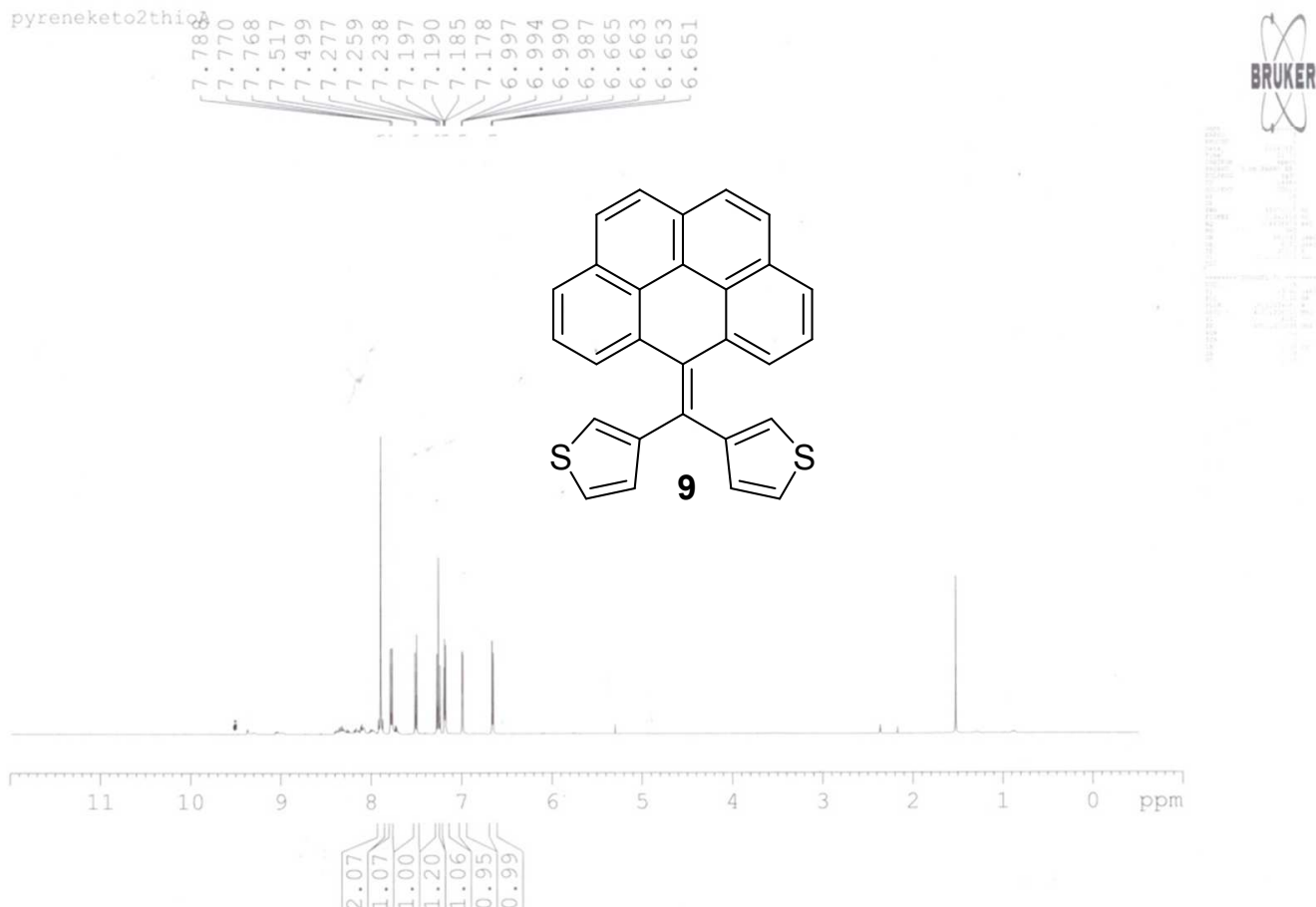

Figure S11. ${ }^{1} \mathrm{H}$ NMR spectrum of 3,3'-((6H-benzo[cd]pyren-6ylidene)methylene)dithiophene (9)
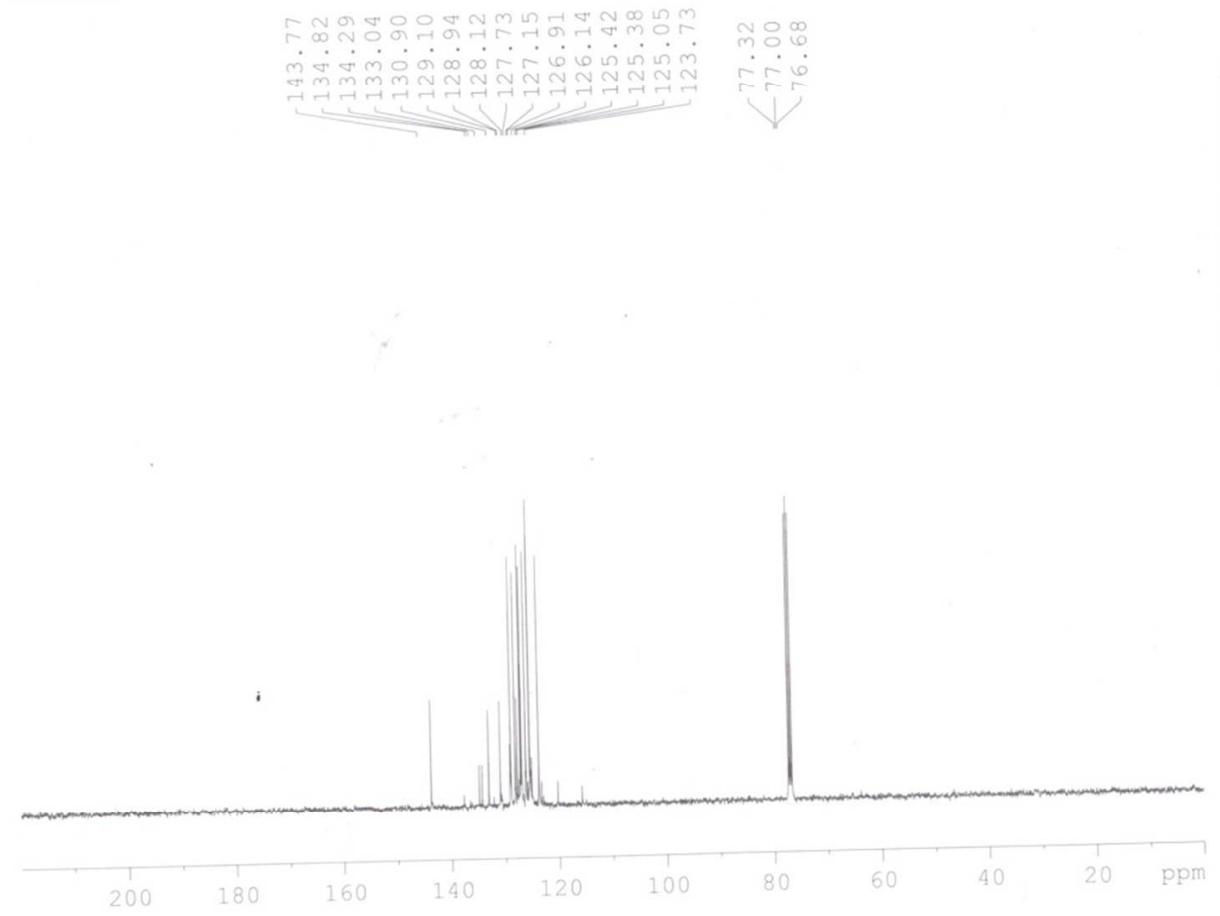

Figure S12. ${ }^{13} \mathrm{C}$ NMR spectrum of 3,3'-((6H-benzo[cd]pyren-6ylidene)methylene)dithiophene (9) 
Supporting Information
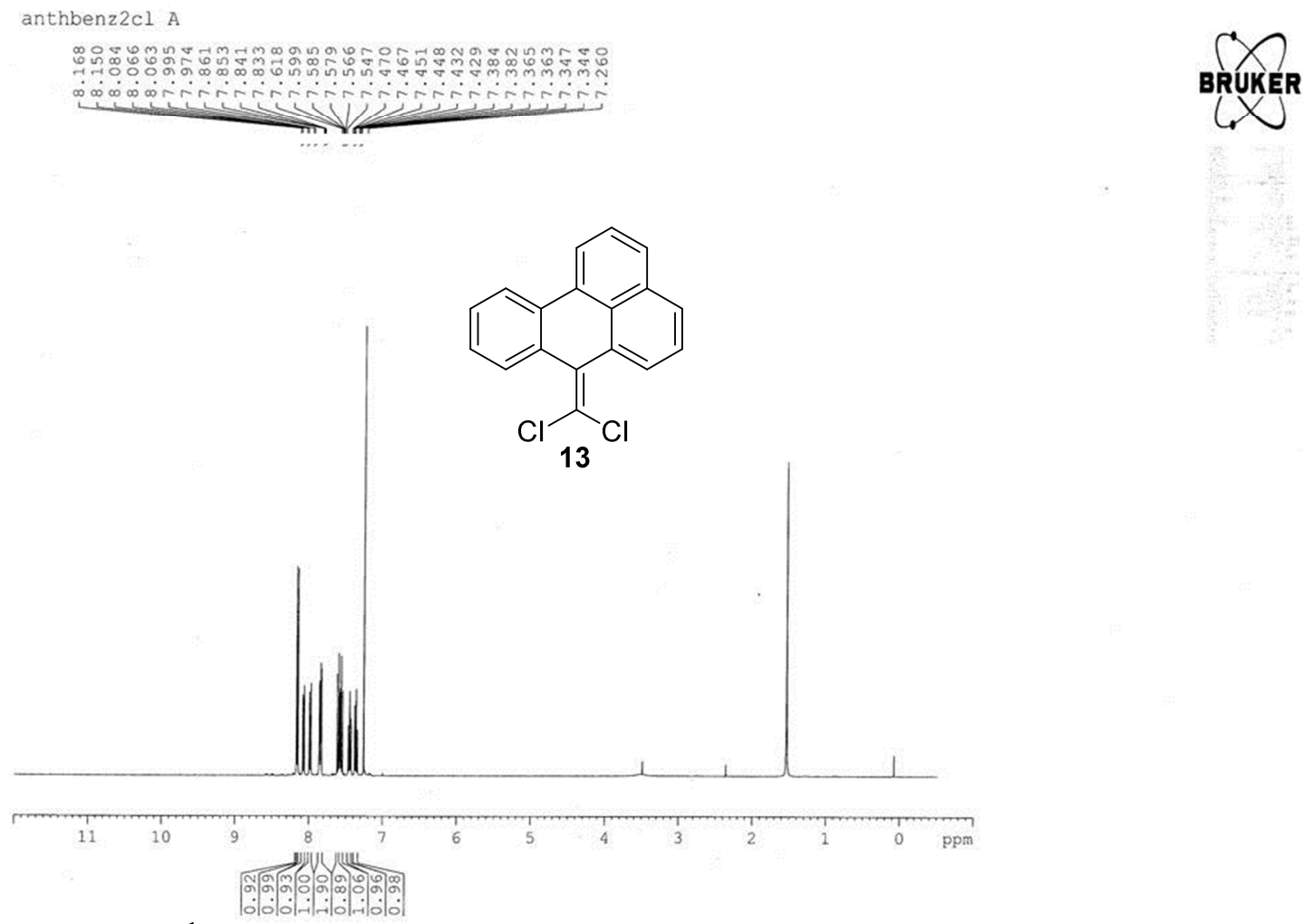

Figure S13. ${ }^{1} \mathrm{H}$ NMR spectrum of 7-(dichloromethylene)-7H-benzo[de $]$ anthracene (13) anthbenz2cl cl:

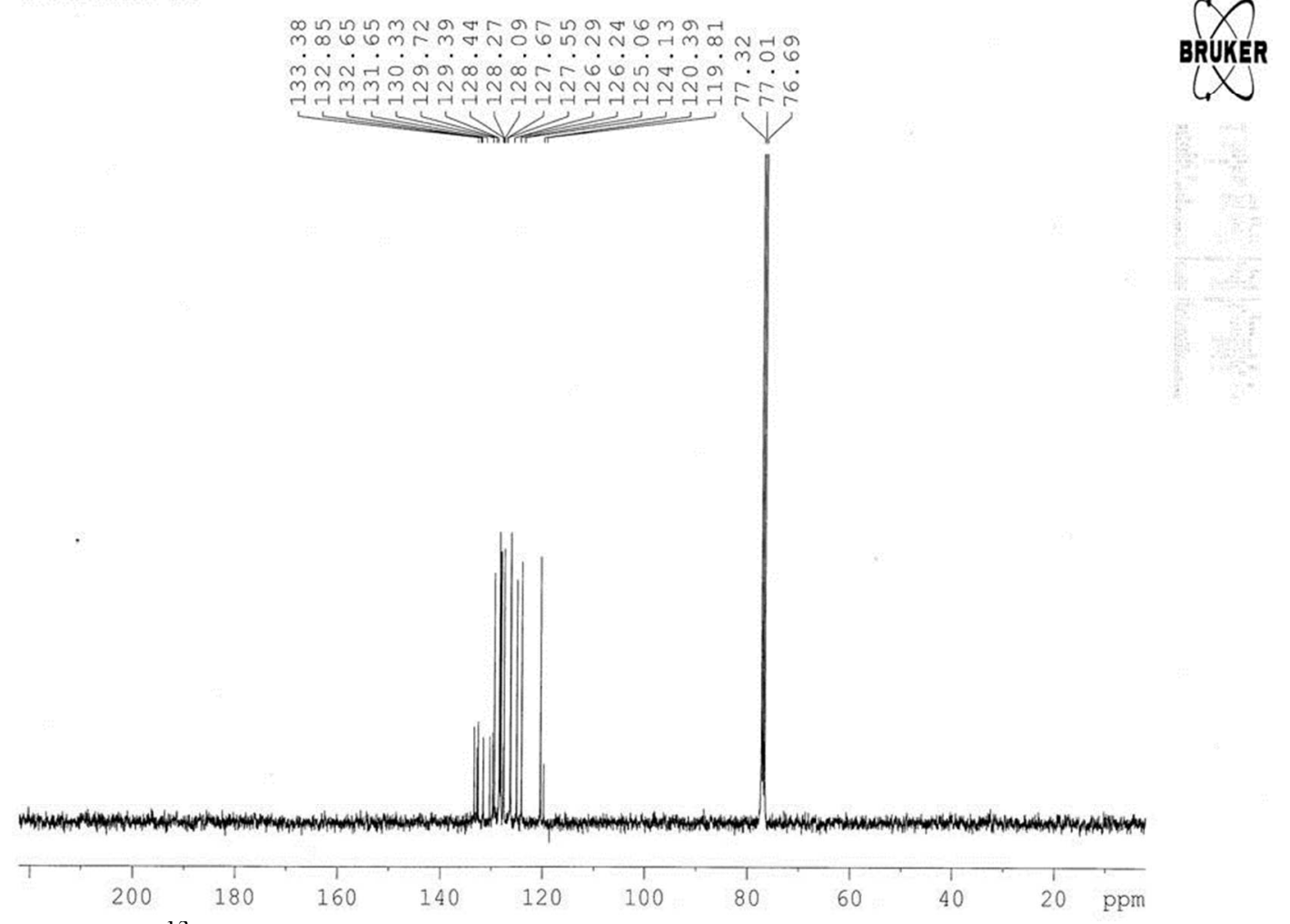

Figure S14. ${ }^{13} \mathrm{C}$ NMR spectrum of 7-(dichloromethylene)-7H-benzo[de]anthracene (13) 
Supporting Information

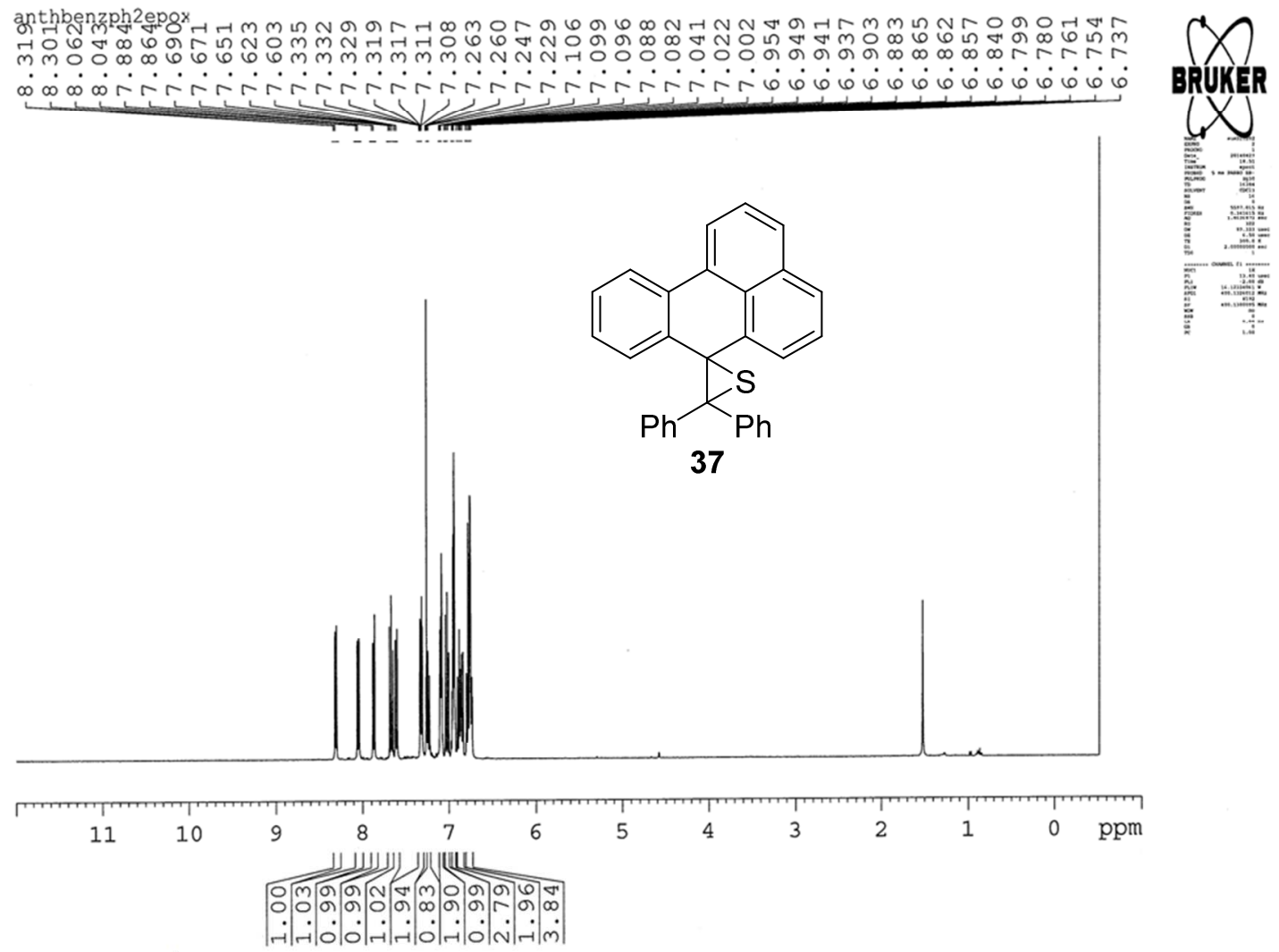

Figure S15. ${ }^{1} \mathrm{H}$ NMR spectrum of 3',3'-diphenylspiro[benzo[de]anthracene-7,2'-thiirane] (37) anthbenz2phthio c1:
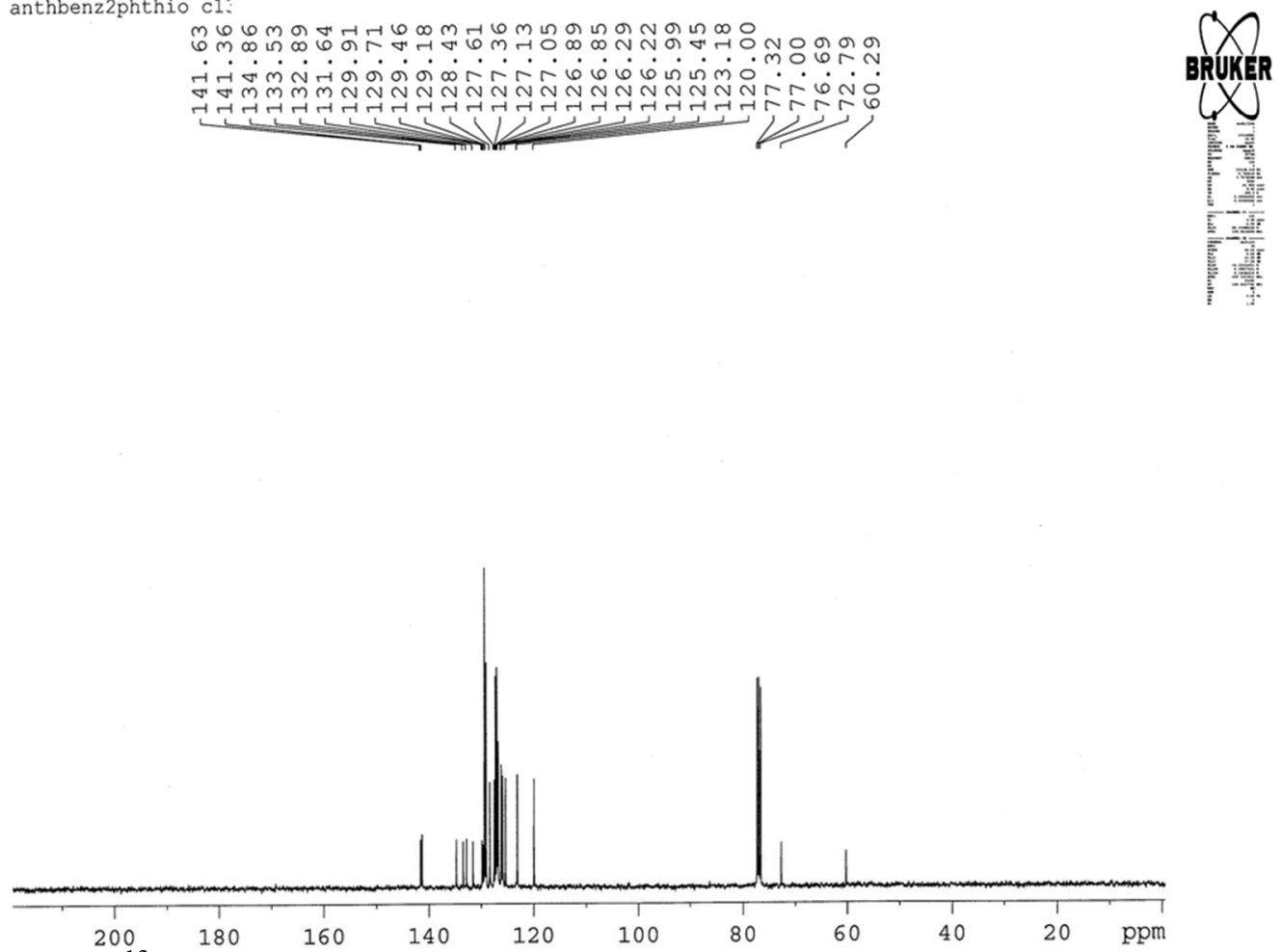

Figure S16. ${ }^{13} \mathrm{C}$ NMR spectrum of 3',3'-diphenylspiro[benzo[de]anthracene-7,2'-thiirane] (37) 


\section{Supporting Information}

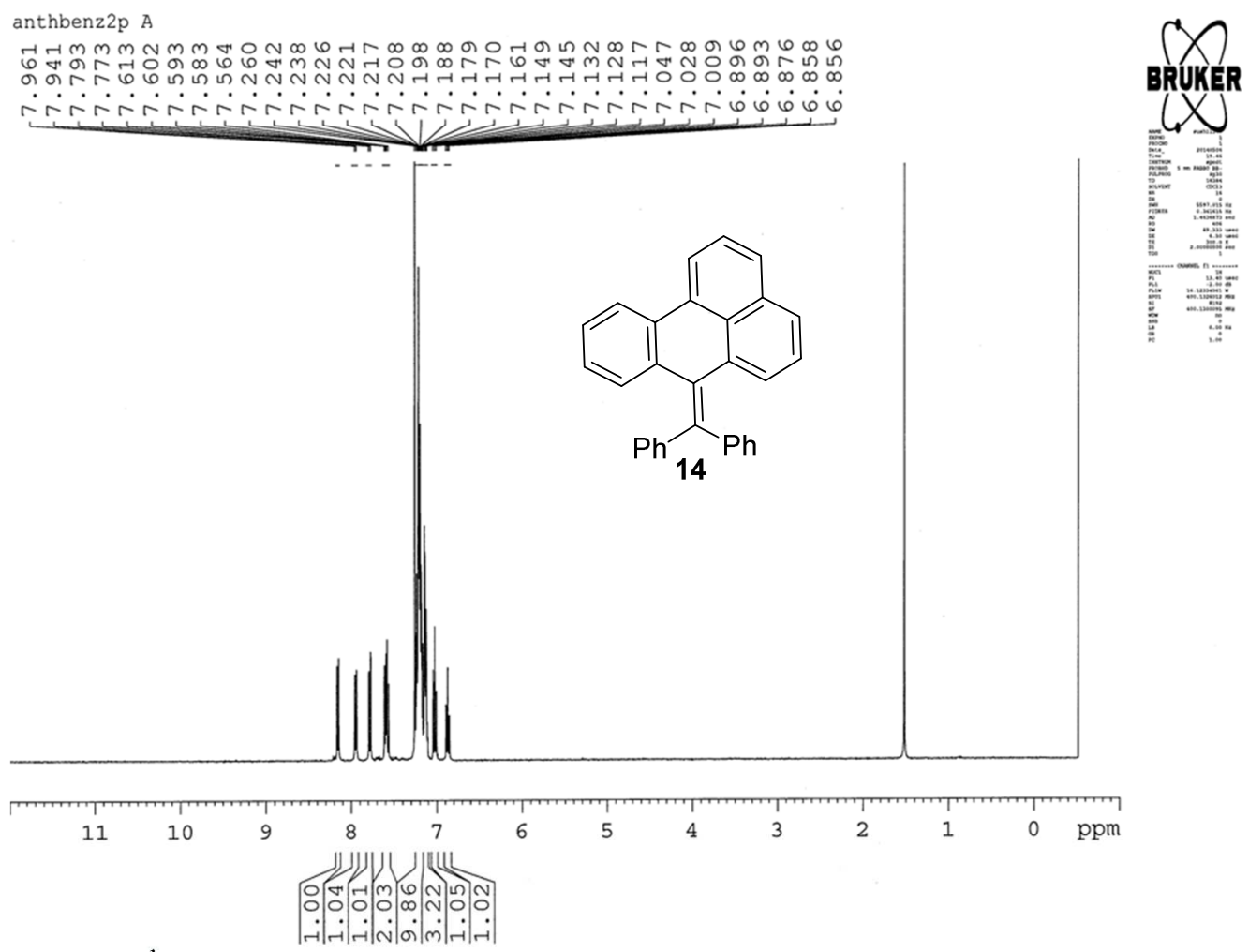

Figure S17. ${ }^{1} \mathrm{H}$ NMR spectrum of 7-(diphenylmethylene)-7H-benzo[de $]$ anthracene (14)

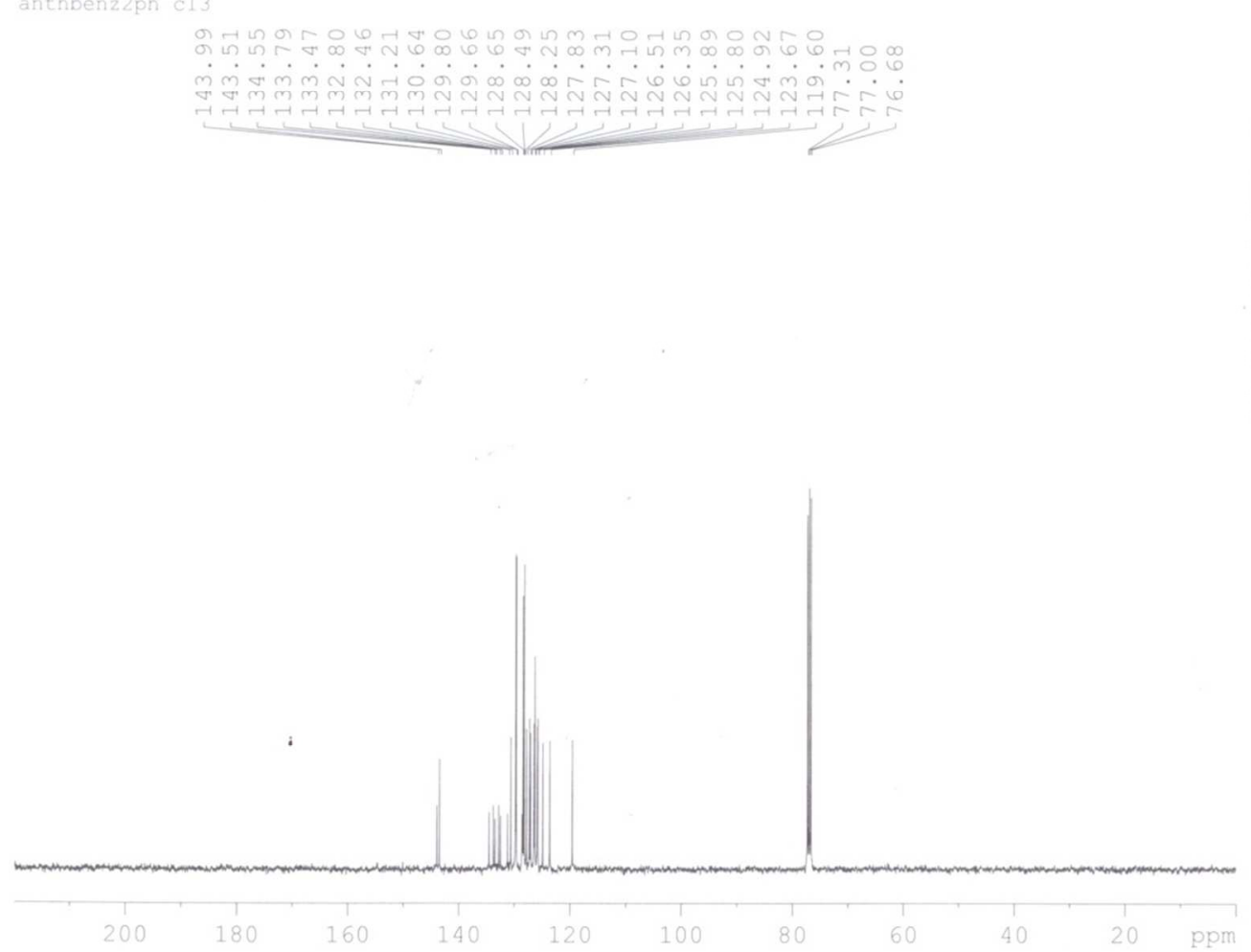

Figure S18. ${ }^{13} \mathrm{C}$ NMR spectrum of 7-(diphenylmethylene)-7H-benzo[de $]$ anthracene (14) 
Supporting Information

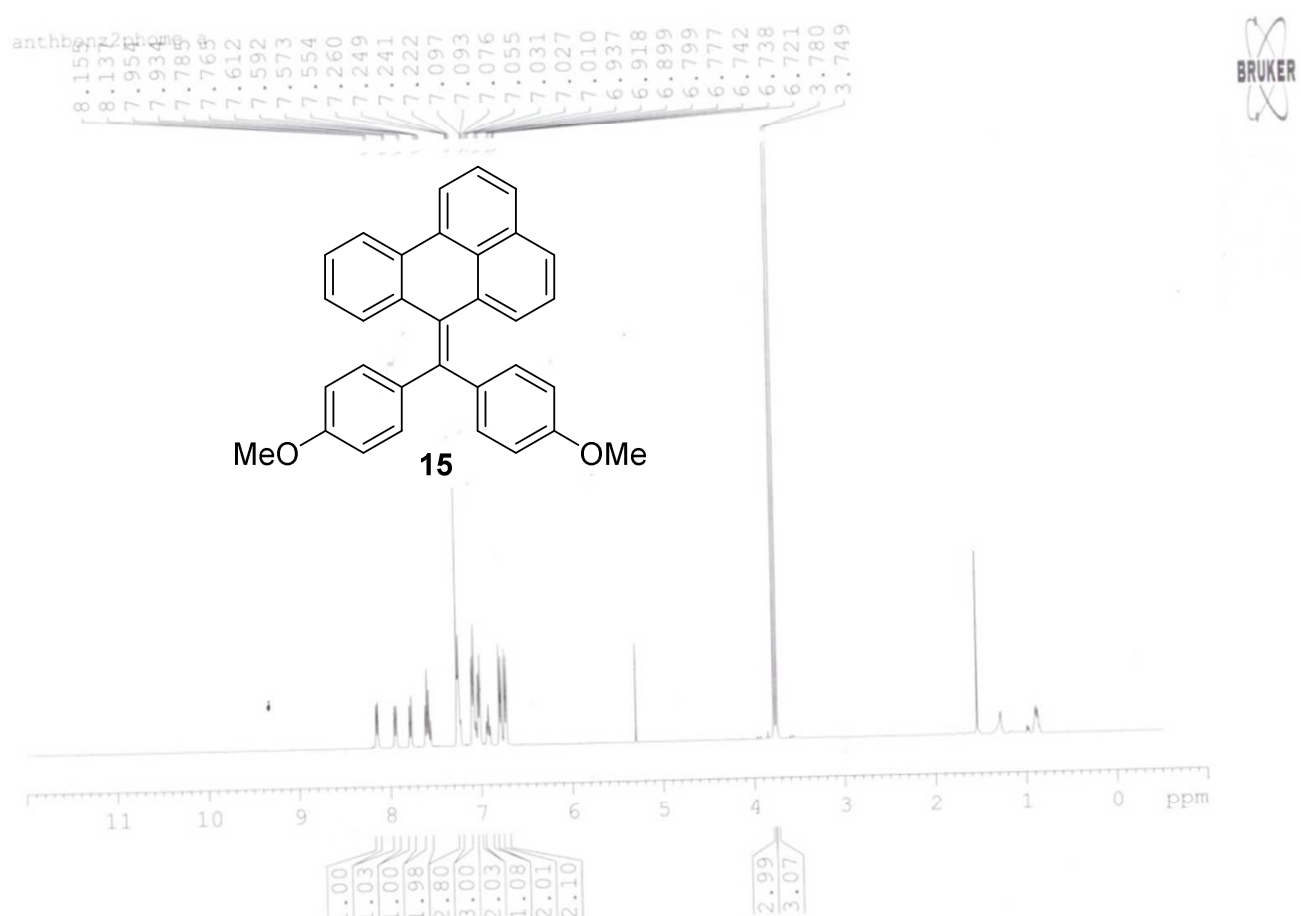

Figure S19. ${ }^{1}$ H NMR spectrum of 7-(bis(4-methoxyphenyl)methylene)-7Hbenzo[de]anthracene (15)

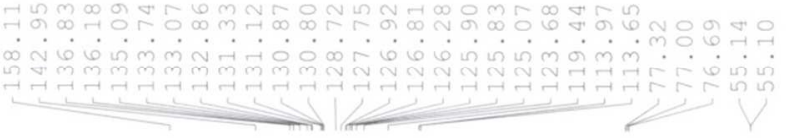

BRUKER
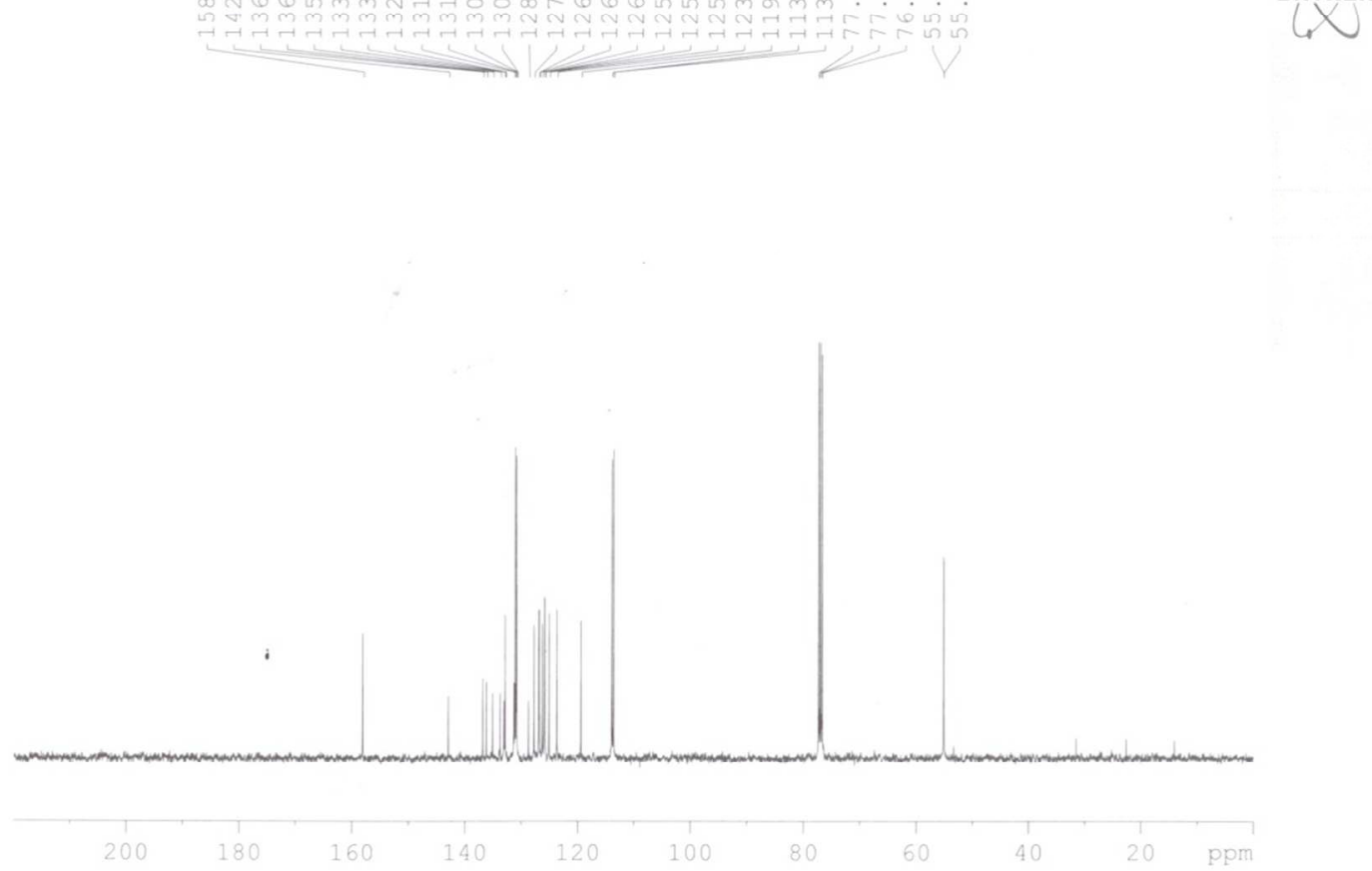

Figure S20. ${ }^{13} \mathrm{C}$ NMR spectrum of of 7-(bis(4-methoxyphenyl)methylene)-7Hbenzo[de]anthracene (15) 


\section{Supporting Information}

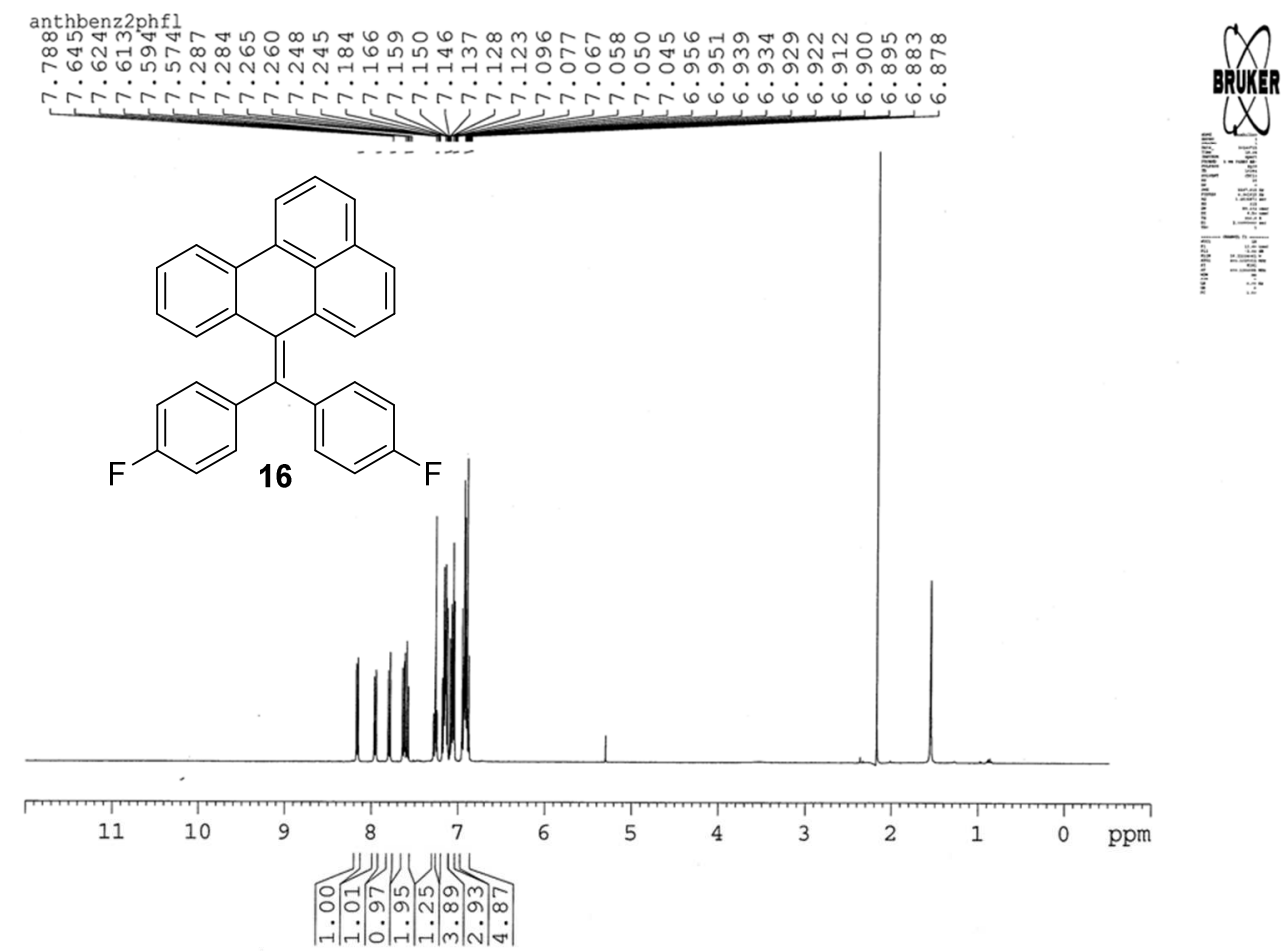

Figure S21. ${ }^{1} \mathrm{H}$ NMR spectrum of 7-(bis(4-fluorophenyl)methylene)-7Hbenzo[de]anthracene (16)

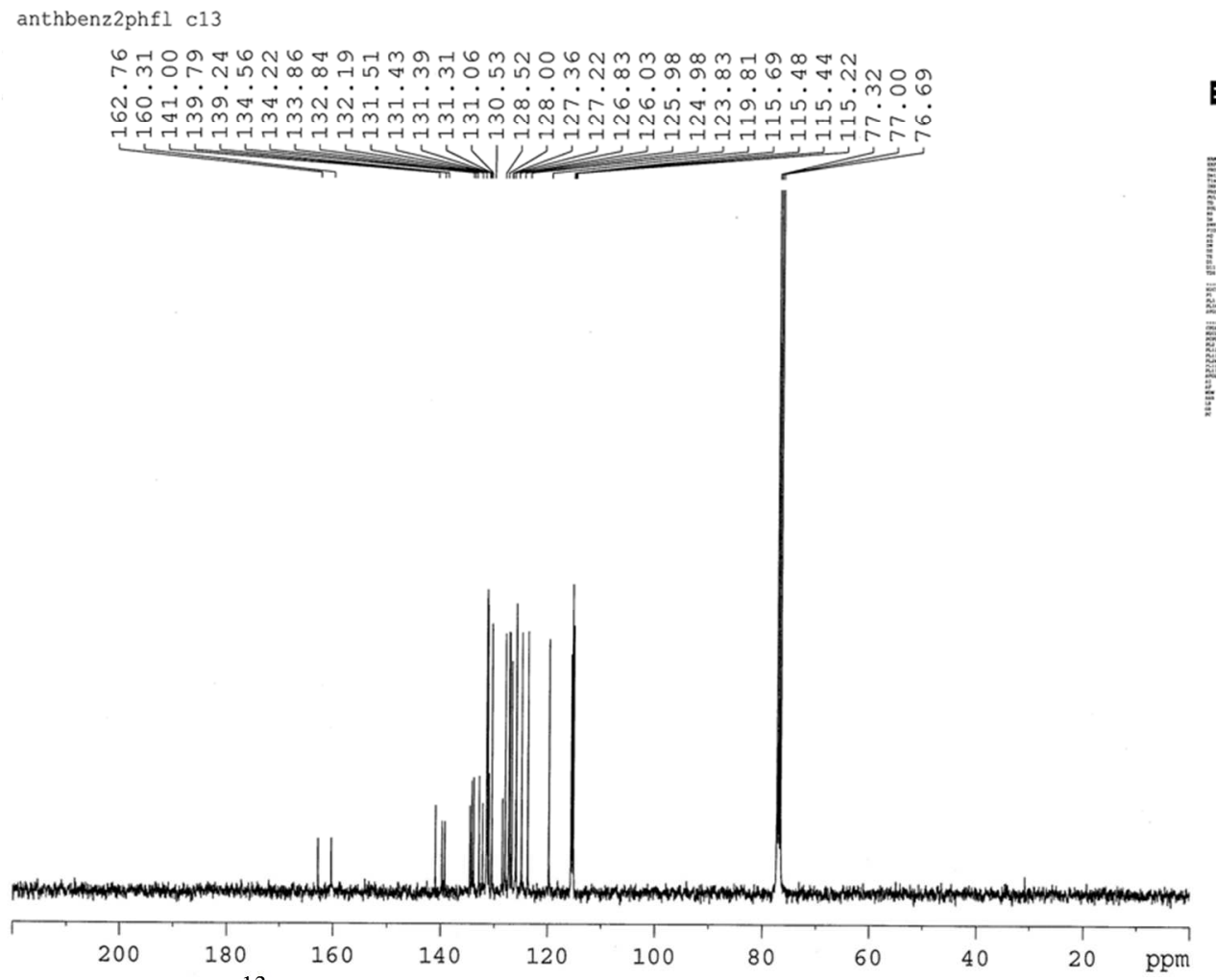

Figure S22. ${ }^{13} \mathrm{C}$ NMR spectrum of of 7-(bis(4-fluorophenyl)methylene)-7Hbenzo[de]anthracene (16) 
Supporting Information

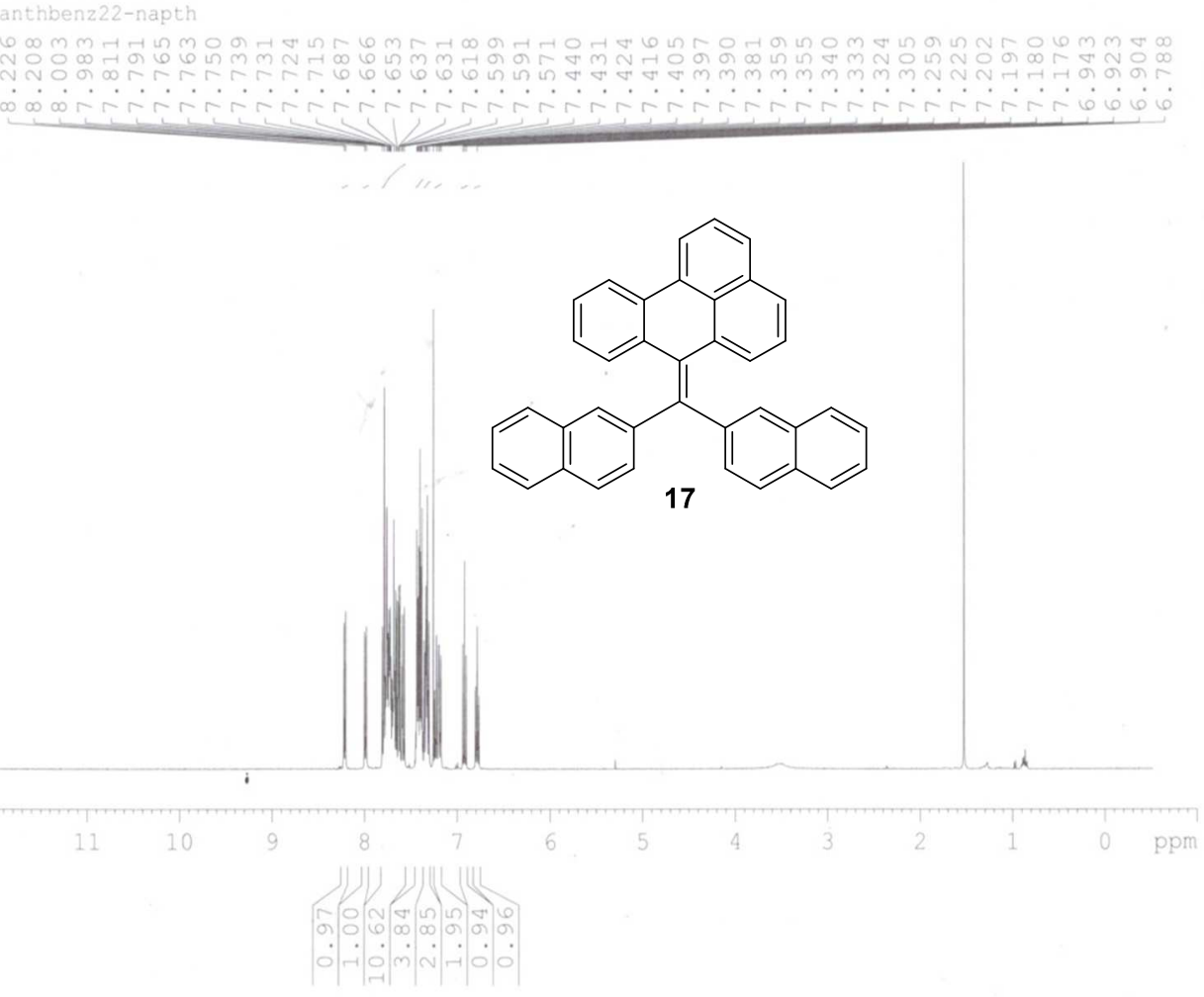

Figure S23. ${ }^{1} \mathrm{H}$ NMR spectrum of 7-(di(naphthalen-2-yl)methylene)-7Hbenzo[de]anthracene (17)

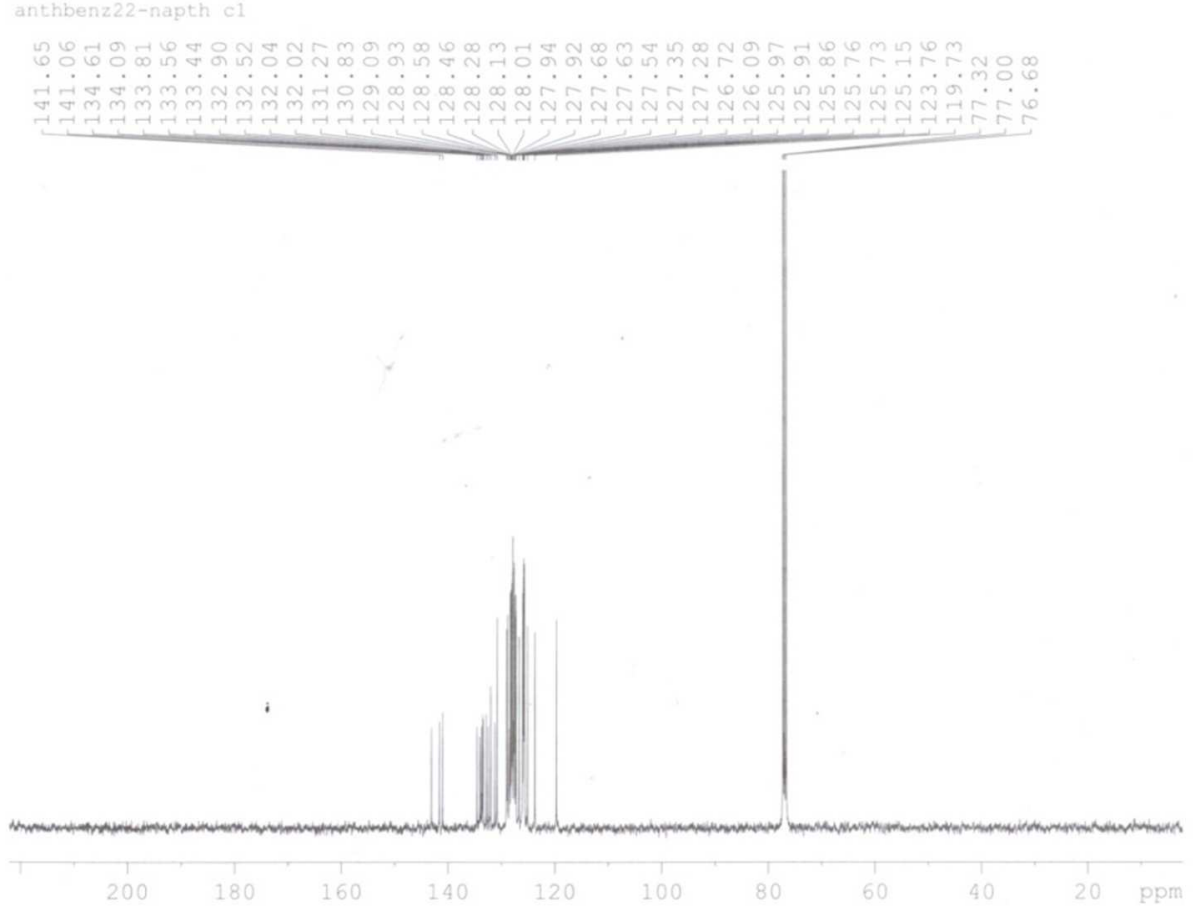

Figure S24. ${ }^{13} \mathrm{C}$ NMR spectrum of 7-(di(naphthalen-2-yl)methylene)-7Hbenzo[de]anthracene (17) 
Supporting Information

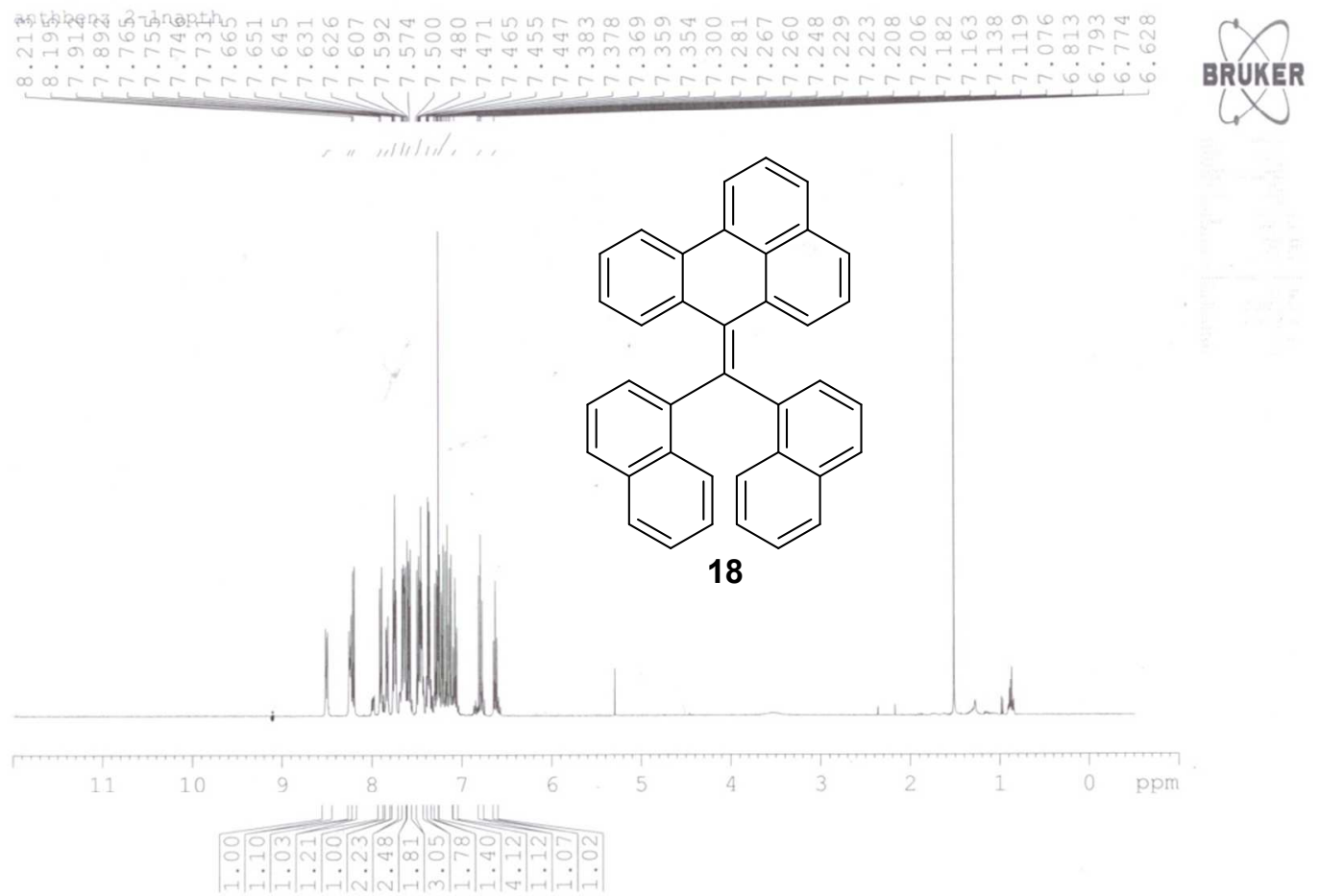

Figure S25. ${ }^{1} \mathrm{H}$ NMR spectrum of 7-(di(naphthalen-1-yl)methylene)-7Hbenzo $[d e]$ anthracene (18)
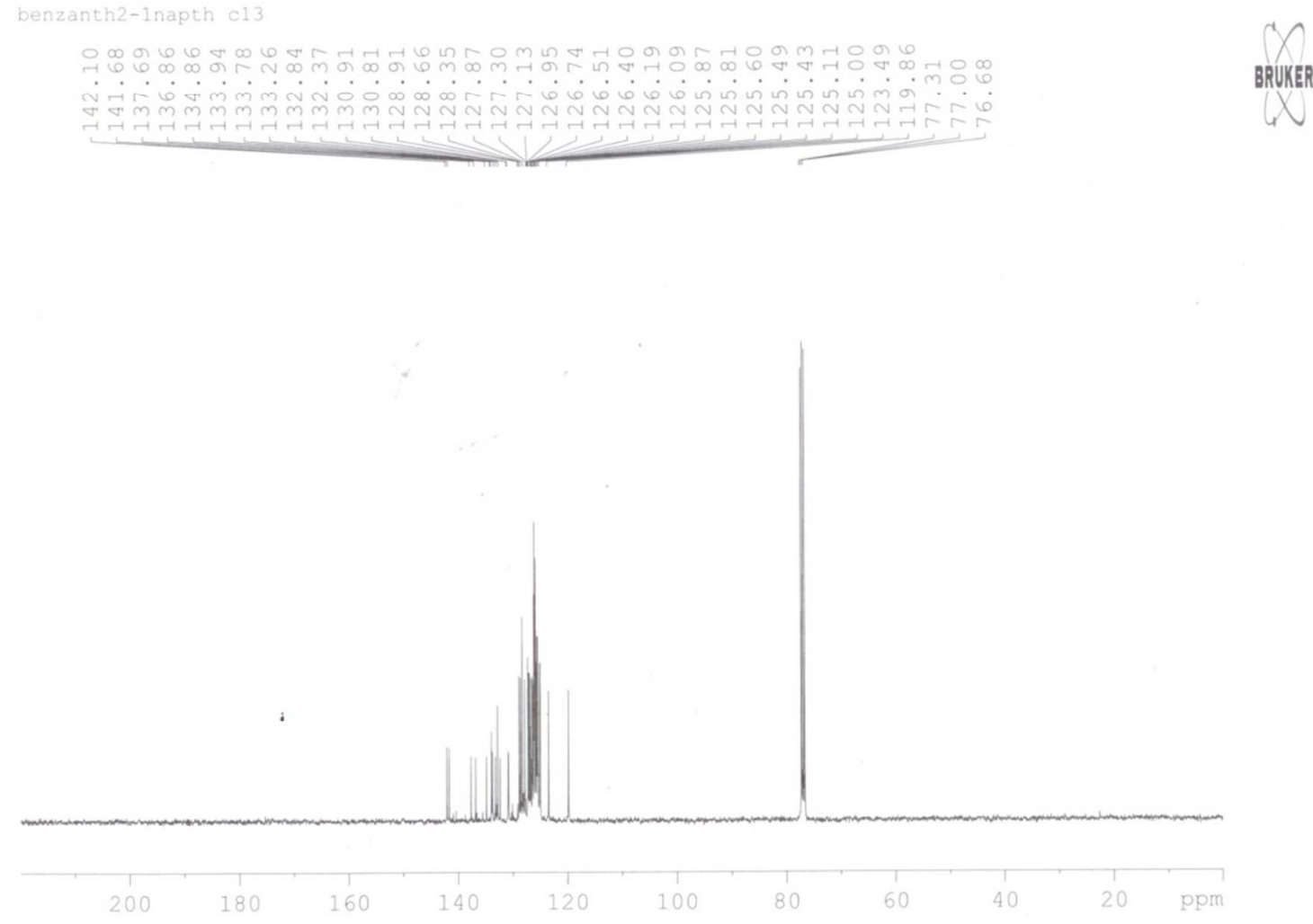

Figure S26. ${ }^{13} \mathrm{C}$ NMR spectrum of 7-(di(naphthalen-1-yl)methylene)-7Hbenzo[de]anthracene (18) 


\section{Supporting Information}

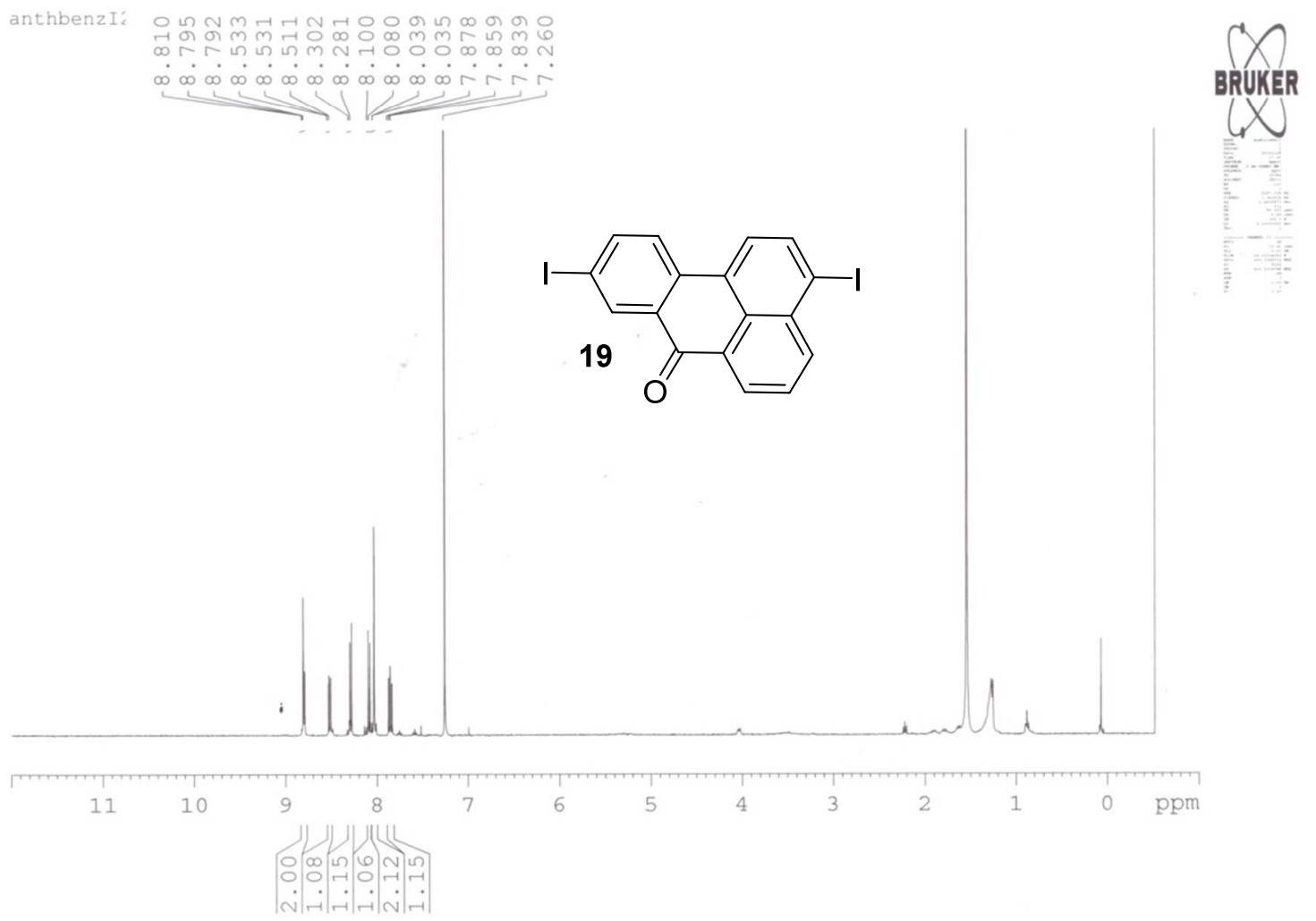

Figure S27. ${ }^{1} \mathrm{H}$ NMR spectrum of 3,9-diiodo-7H-benzo[de]anthracen-7-one (19) benzanth ph

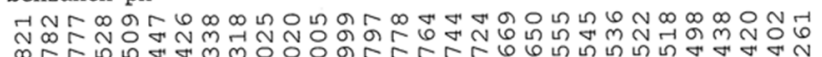

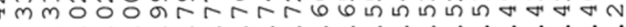

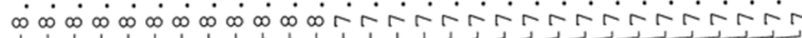

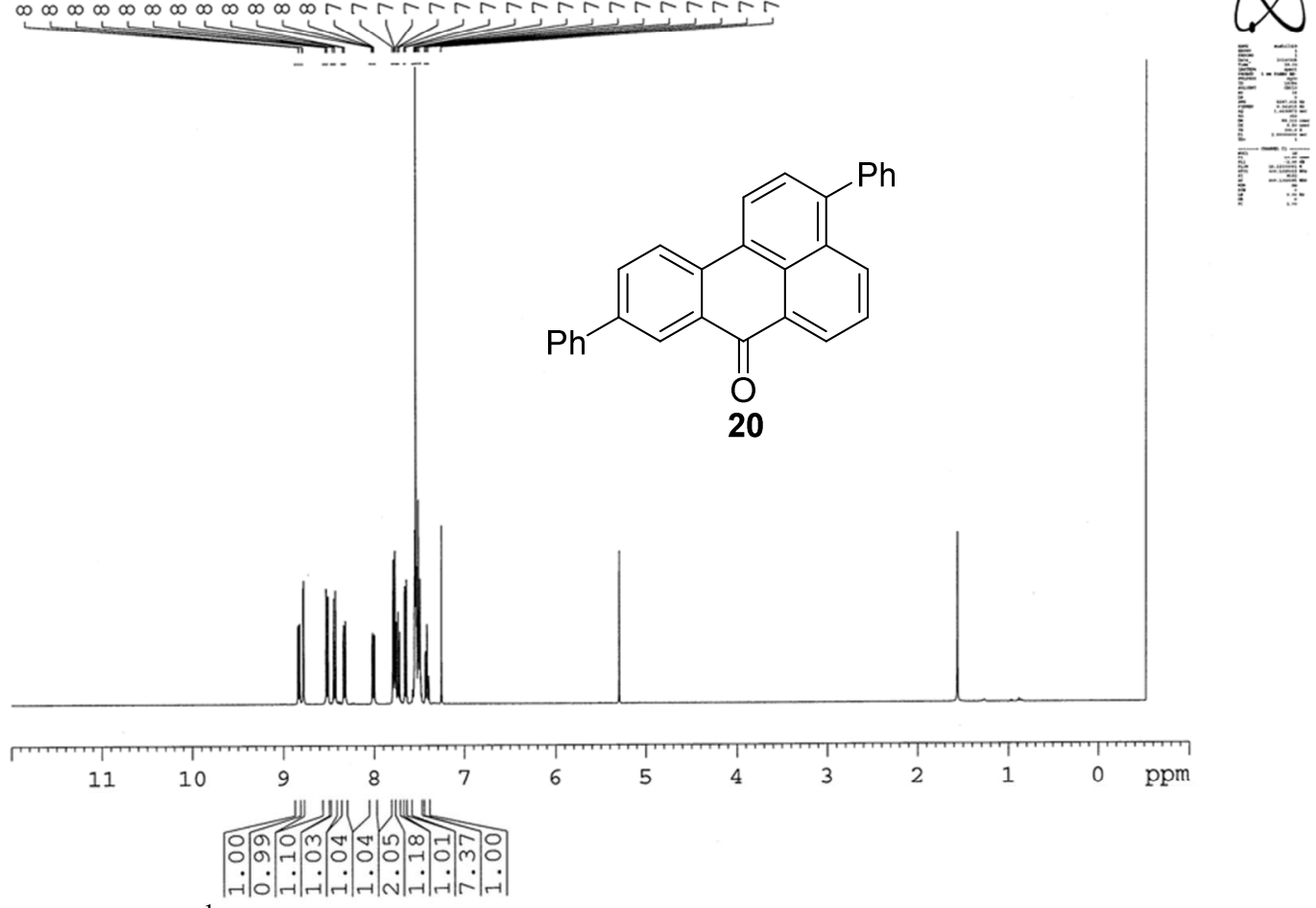

Figure S28. ${ }^{1} \mathrm{H}$ NMR spectra of 3,9-diphenyl-7H-benzo[de $]$ anthracen-7-one (20) 
Supporting Information

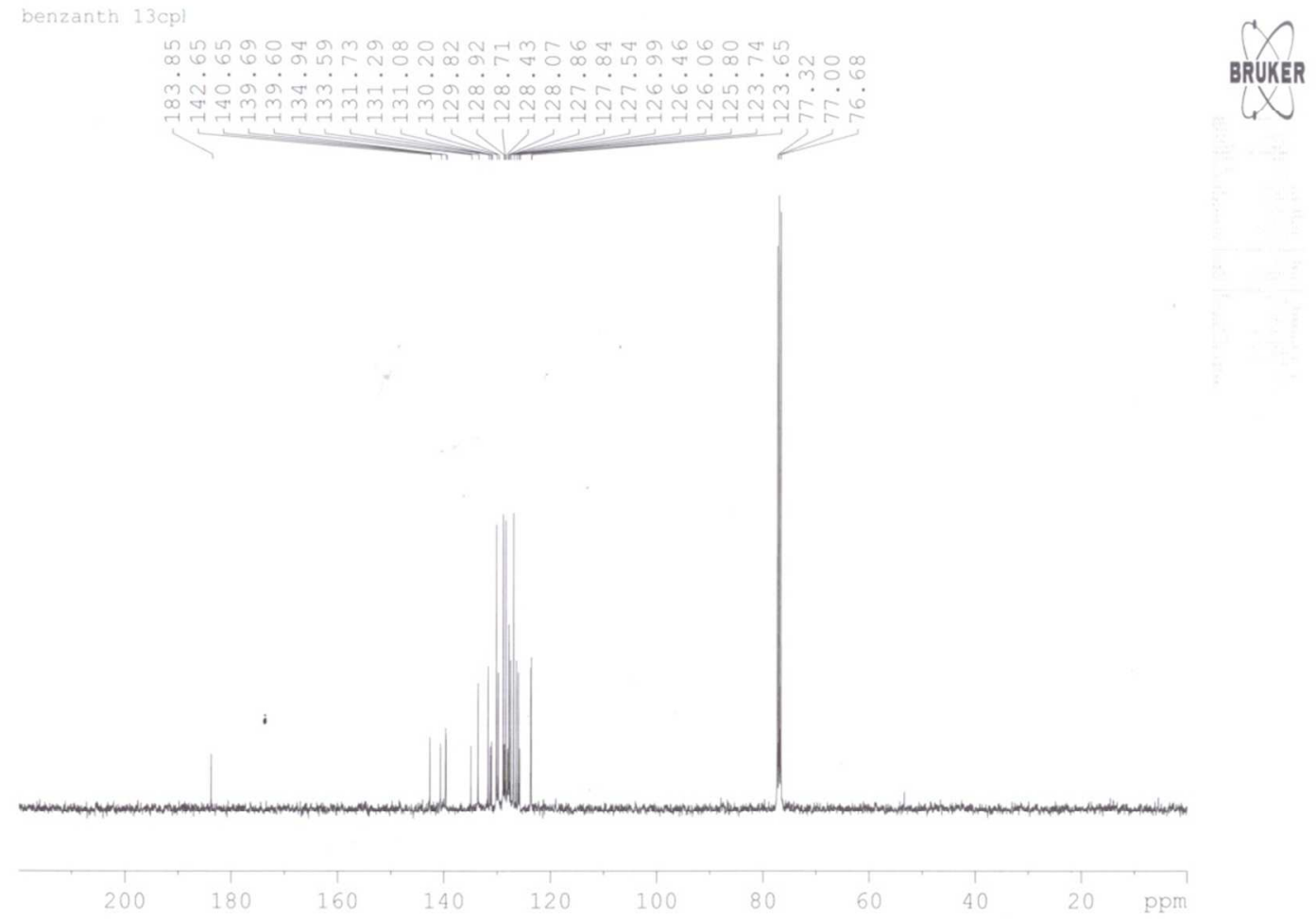

Figure S29. ${ }^{13} \mathrm{C}$ NMR spectra of 3,9-diphenyl-7H-benzo[de $]$ anthracen-7-one (20)

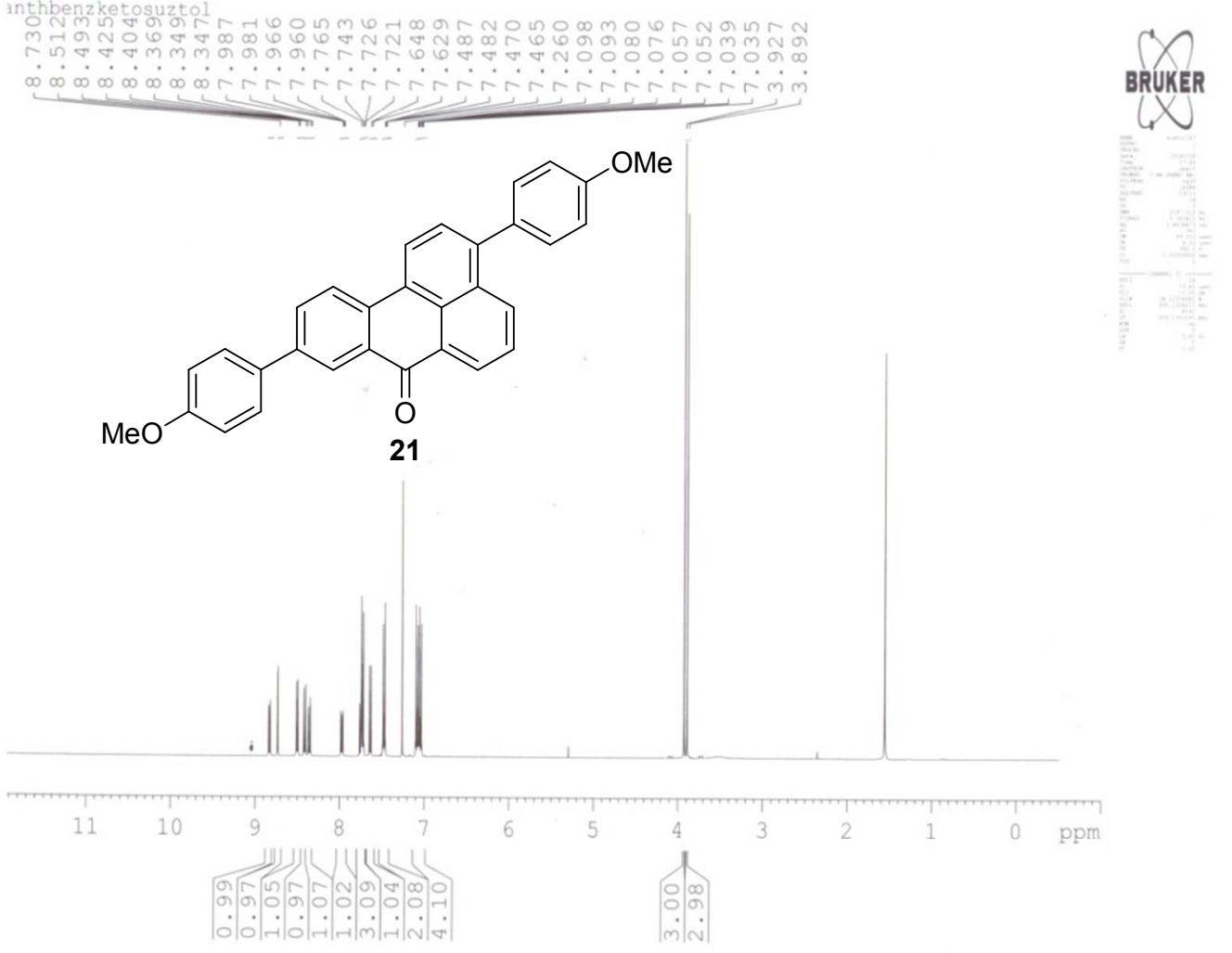

Figure S30. ${ }^{1} \mathrm{H}$ NMR spectrum of 3,9-bis(4-methoxyphenyl)-7H-benzo[de]anthracen-7one (21) 
Supporting Information

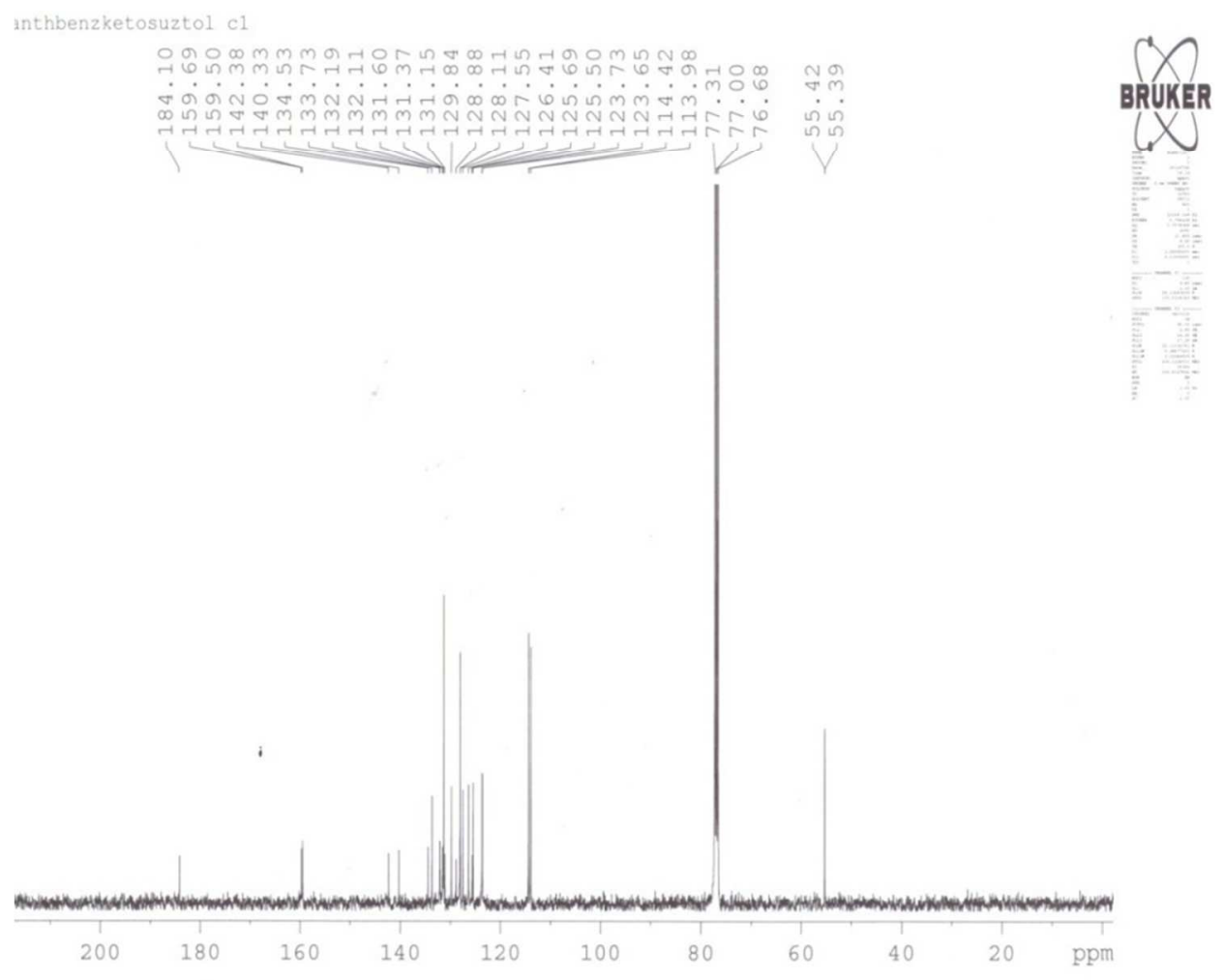

Figure S31. ${ }^{13} \mathrm{C}$ NMR spectrum of 3,9-bis(4-methoxyphenyl)-7H-benzo[de $]$ anthracen-7one (21)

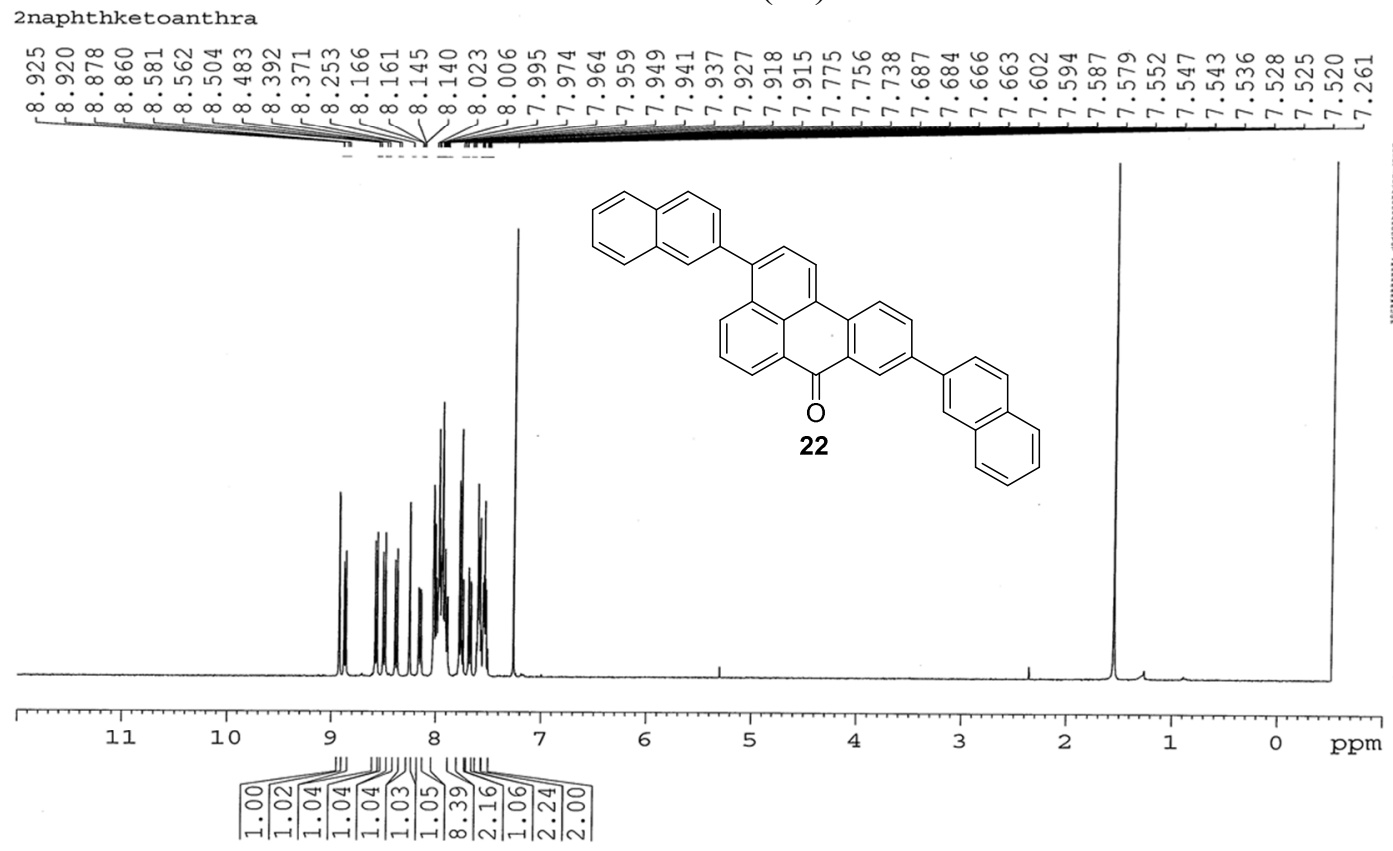

Figure S32. ${ }^{1} \mathrm{H}$ NMR spectrum of 3,9-di(naphthalen-2-yl)-7H-benzo[de $]$ anthracen-7-one (22) 
Supporting Information

2-napthketoc13

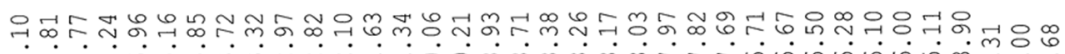

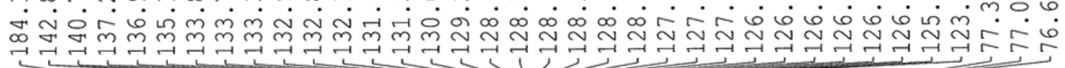

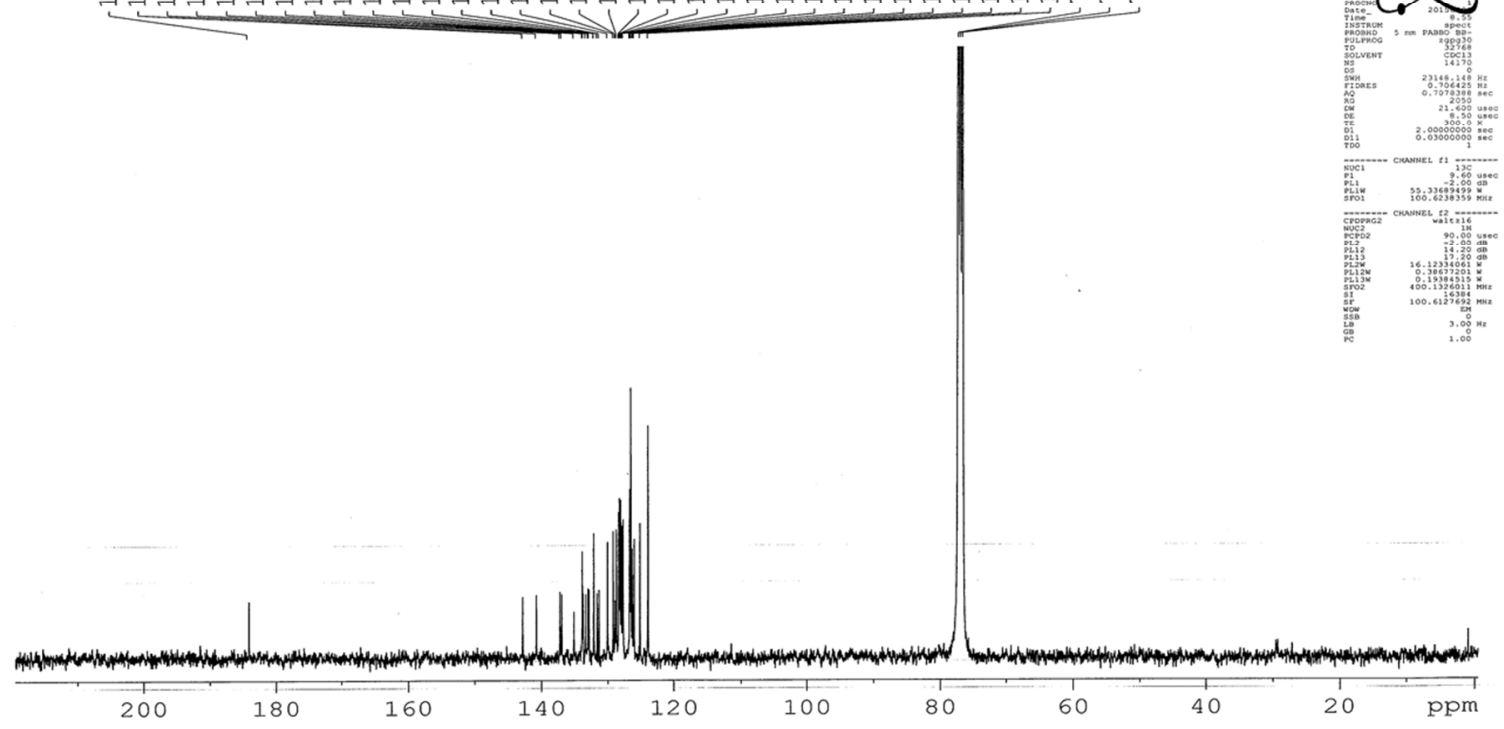

Figure S33. ${ }^{13} \mathrm{C}$ NMR spectrum of 3,9-di(naphthalen-2-yl)-7H-benzo[de]anthracen-7-one (22)

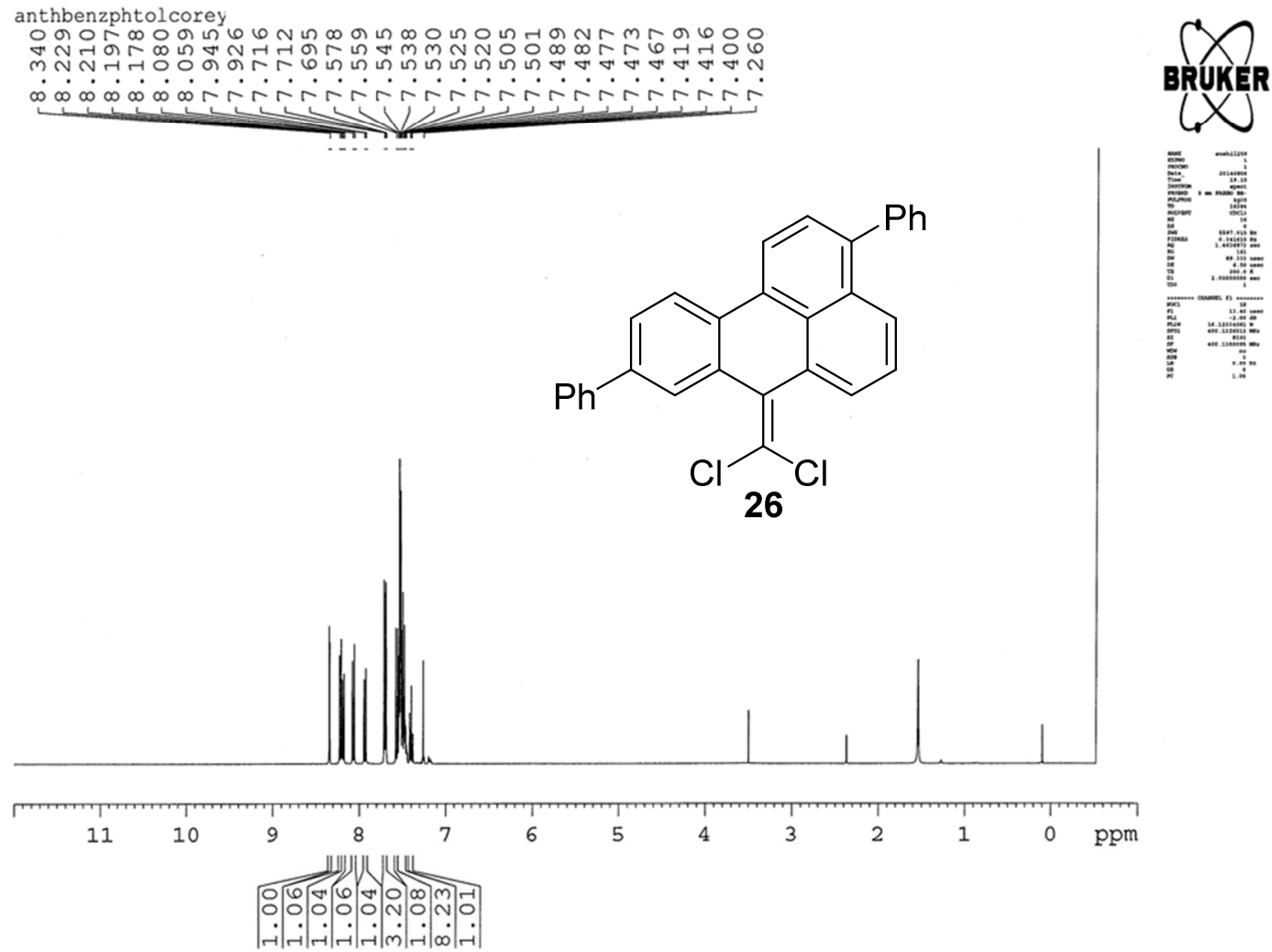

Figure S34. ${ }^{1} \mathrm{H}$ NMR spectrum of 7-(dichloromethylene)-3,9-diphenyl-7Hbenzo[de]anthracene (26) 
Supporting Information

anthbenz2ph2cl corey c13

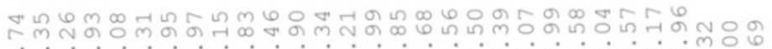

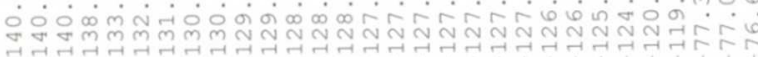

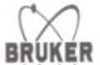

(x)

$=$

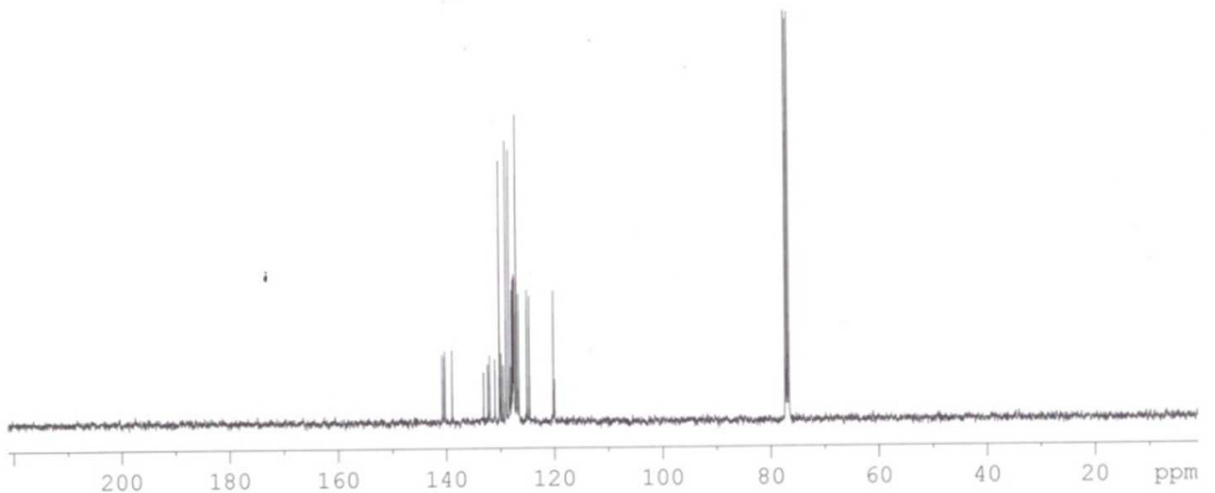

Figure S35. ${ }^{13} \mathrm{C}$ NMR spectrum of 7-(dichloromethylene)-3,9-diphenyl-7Hbenzo[de]anthracene (26)

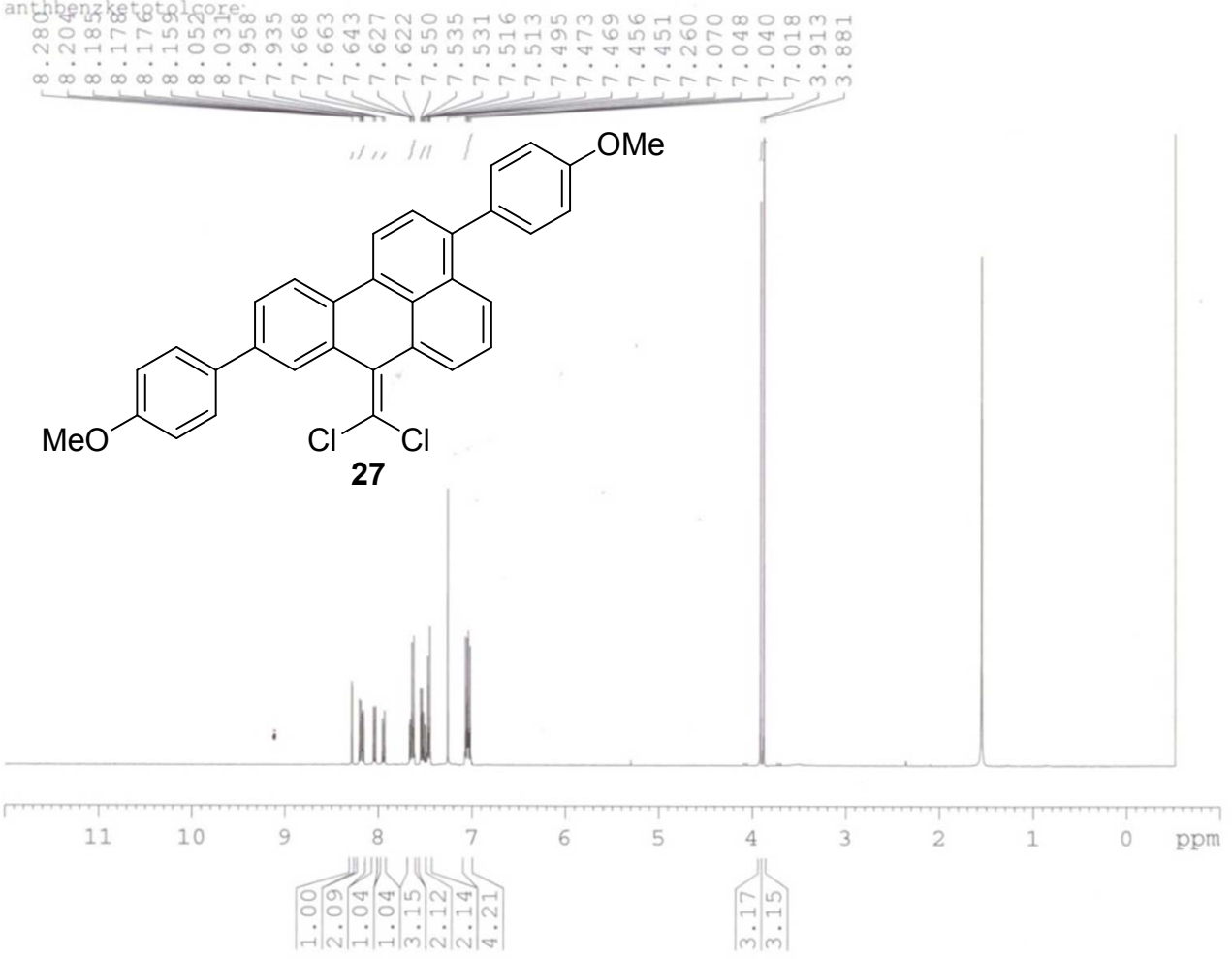

Figure S36. ${ }^{1} \mathrm{H}$ NMR spectrum of 7-(dichloromethylene)-3,9-bis(4-methoxyphenyl)-7Hbenzo[de]anthracene (27) 
Supporting Information

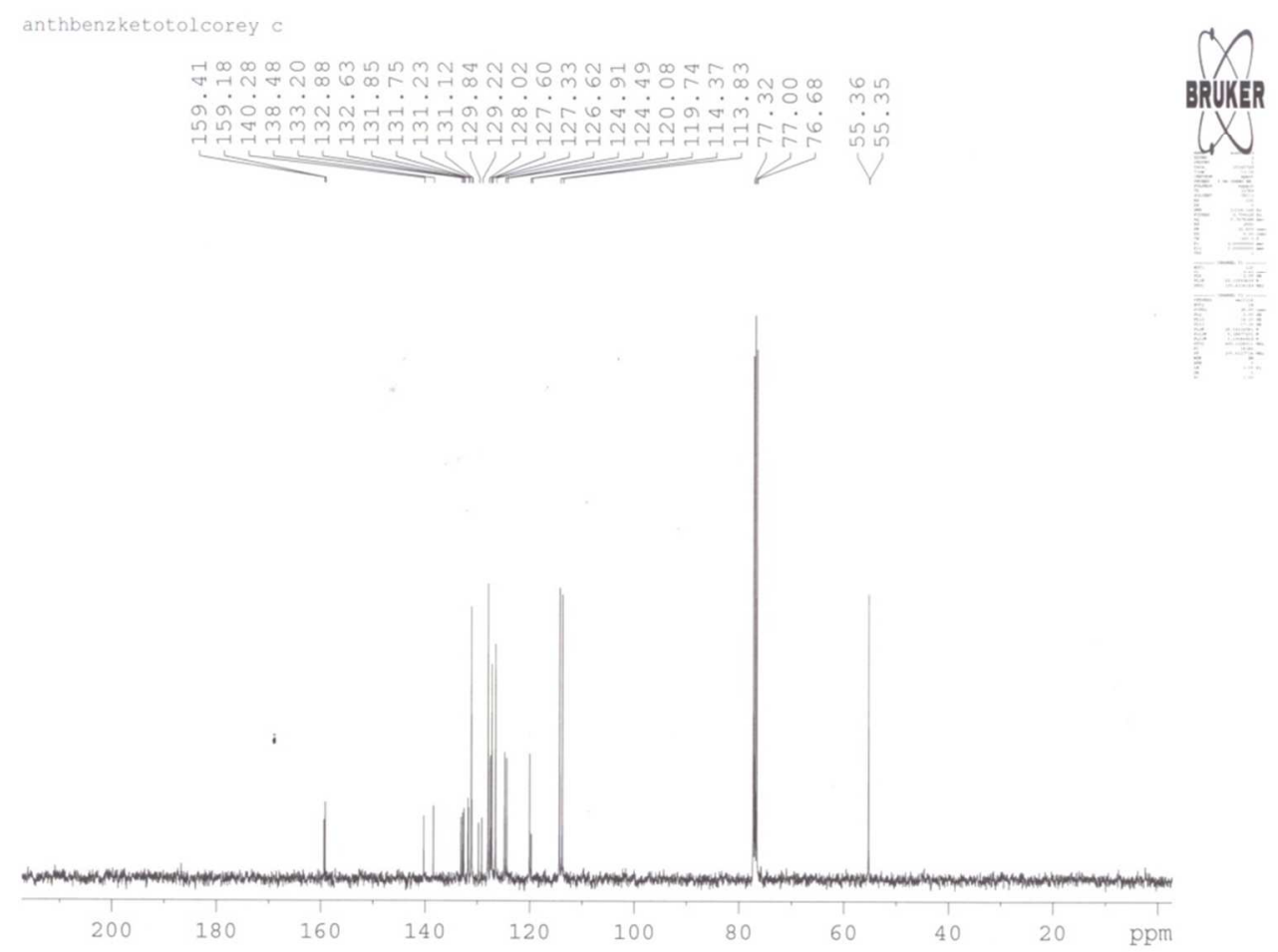

Figure S37. ${ }^{13} \mathrm{C}$ NMR spectrum of 7-(dichloromethylene)-3,9-bis(4-methoxyphenyl)-7Hbenzo[de]anthracene (27)

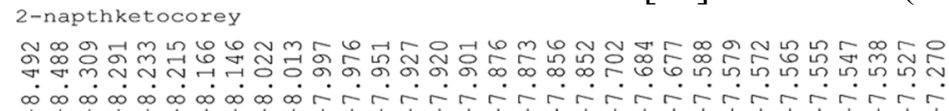

শ

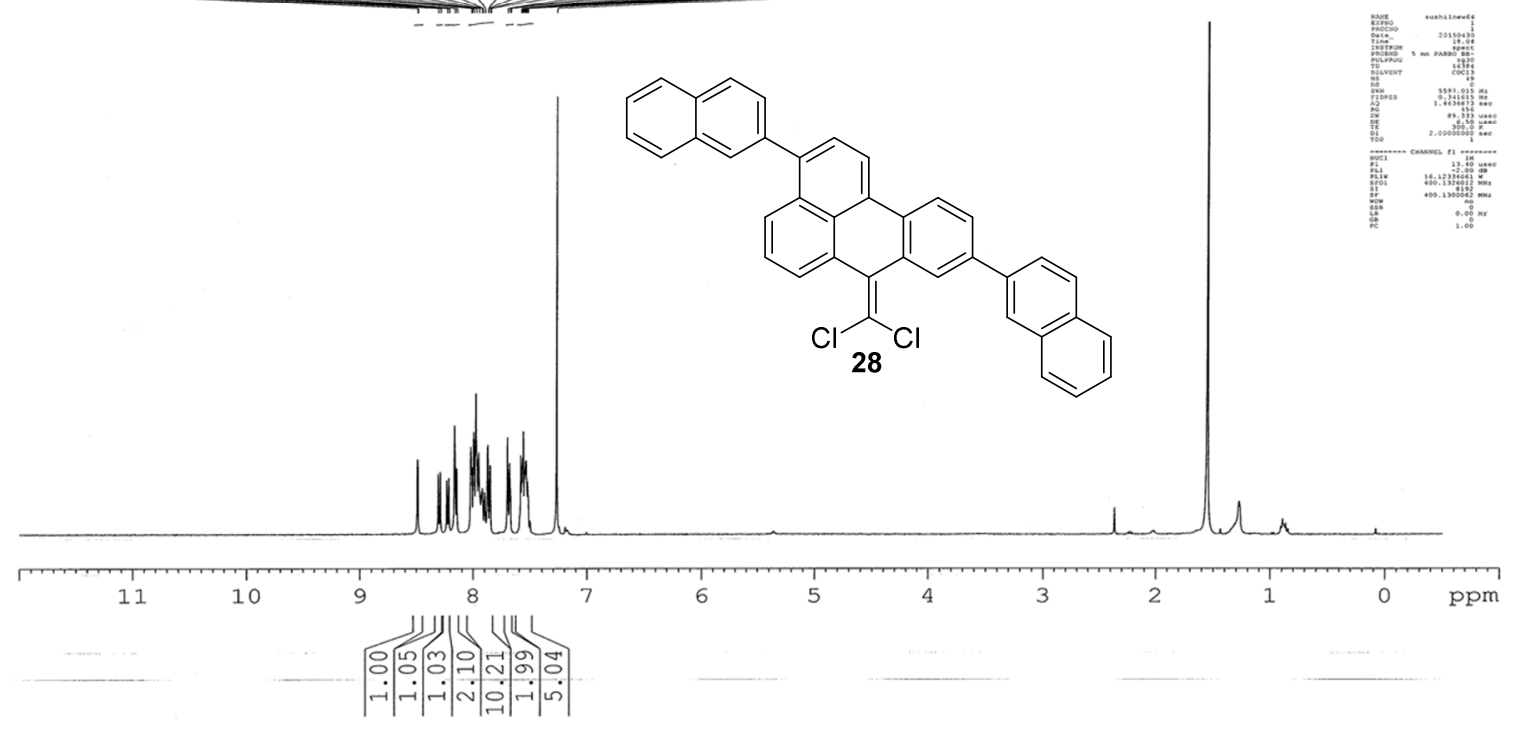

Figure S38. ${ }^{1} \mathrm{H}$ NMR spectrum of 7-(dichloromethylene)-3,9-di(naphthalen-2-yl)-7Hbenzo $[d e]$ anthracene (28) 
Supporting Information

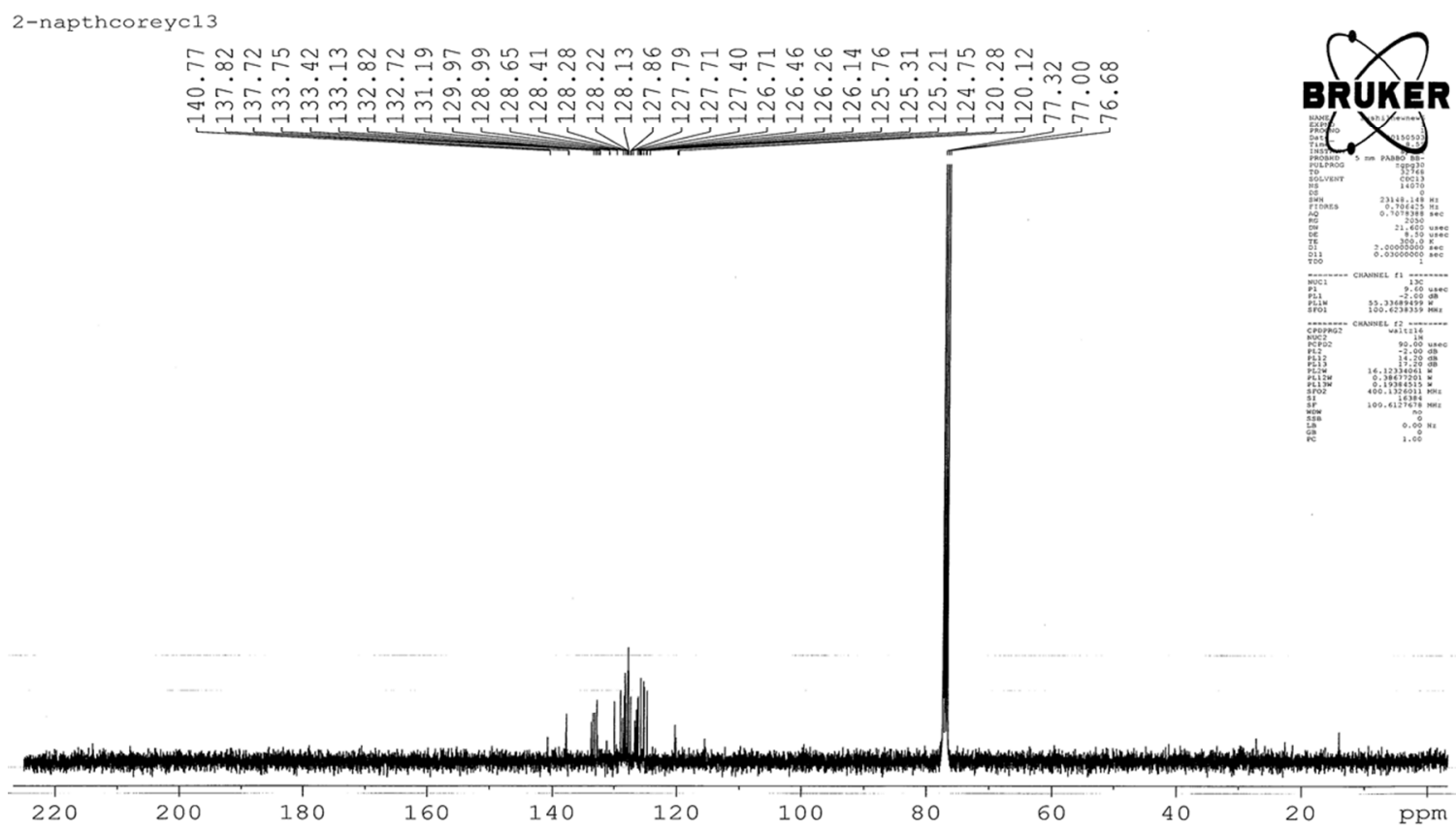

Figure S39. ${ }^{13} \mathrm{C}$ NMR spectrum of 7-(dichloromethylene)-3,9-di(naphthalen-2-yl)-7Hbenzo[de]anthracene (28)

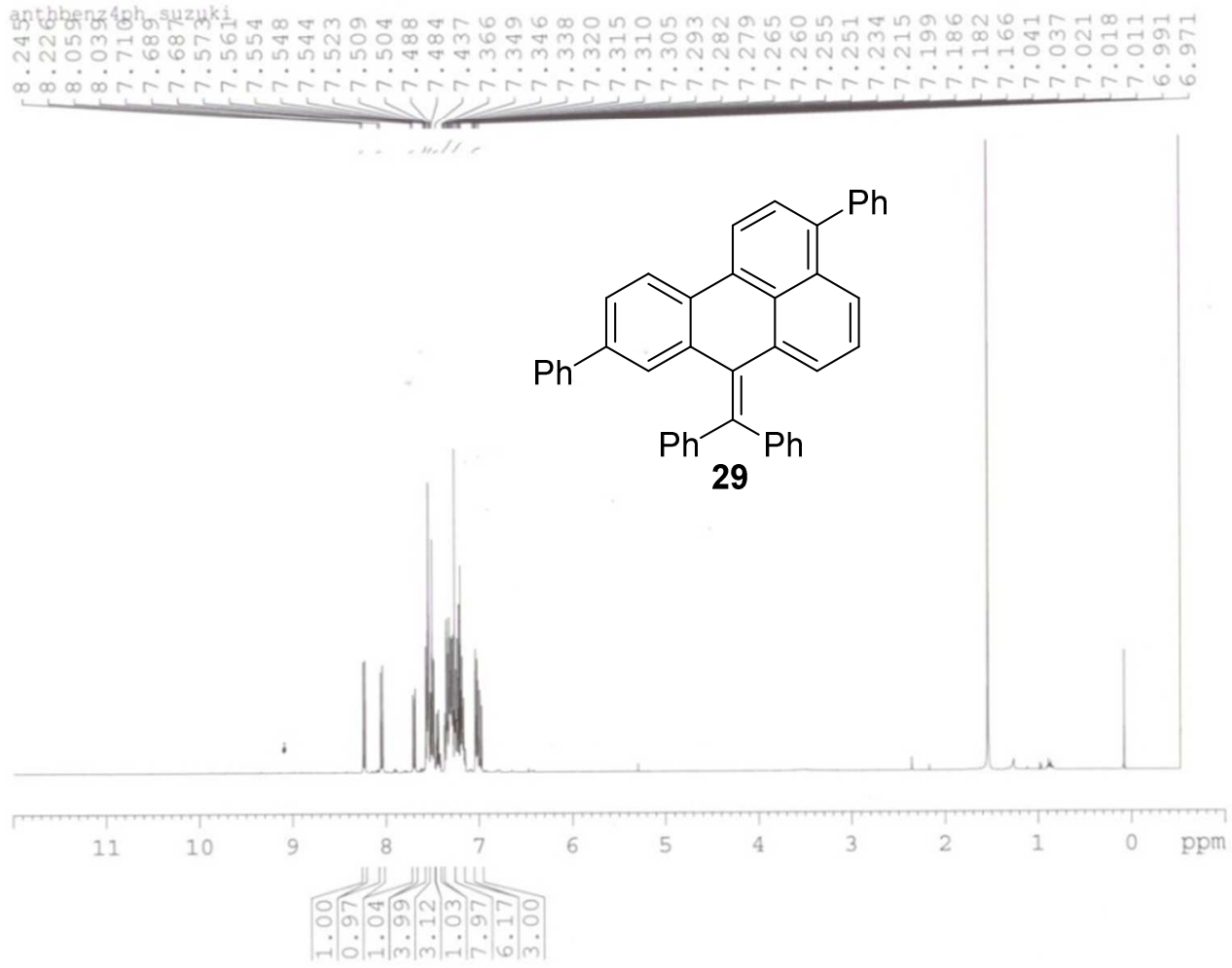

Figure S40. ${ }^{1}$ H NMR spectrum of 7-(diphenylmethylene)-3,9-diphenyl-7Hbenzo[de]anthracene (29) 
Supporting Information

anthbenz4ph suzuki $\mathrm{cl}$

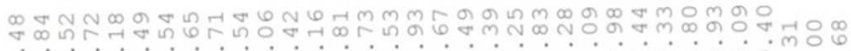

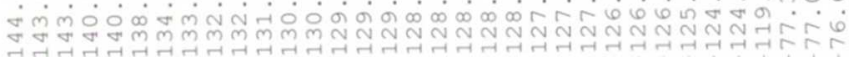

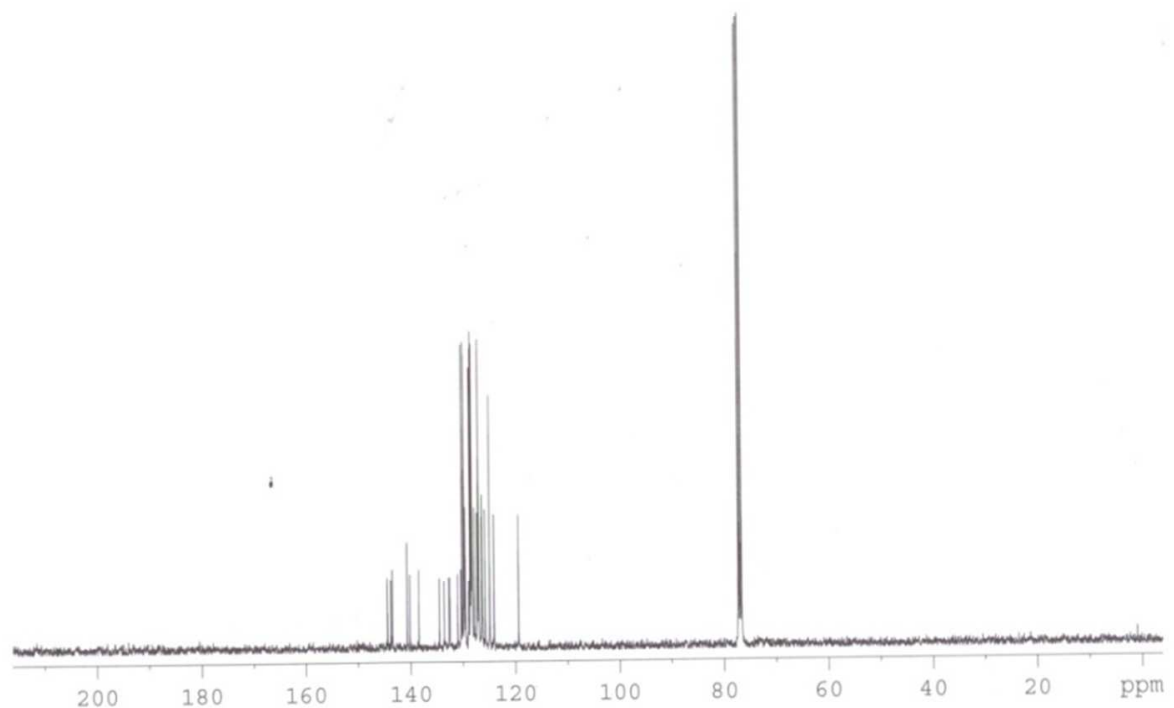

Figure S41. ${ }^{13} \mathrm{C}$ NMR spectrum of 7-(diphenylmethylene)-3,9-diphenyl-7Hbenzo[ $[d e]$ anthracene (29)

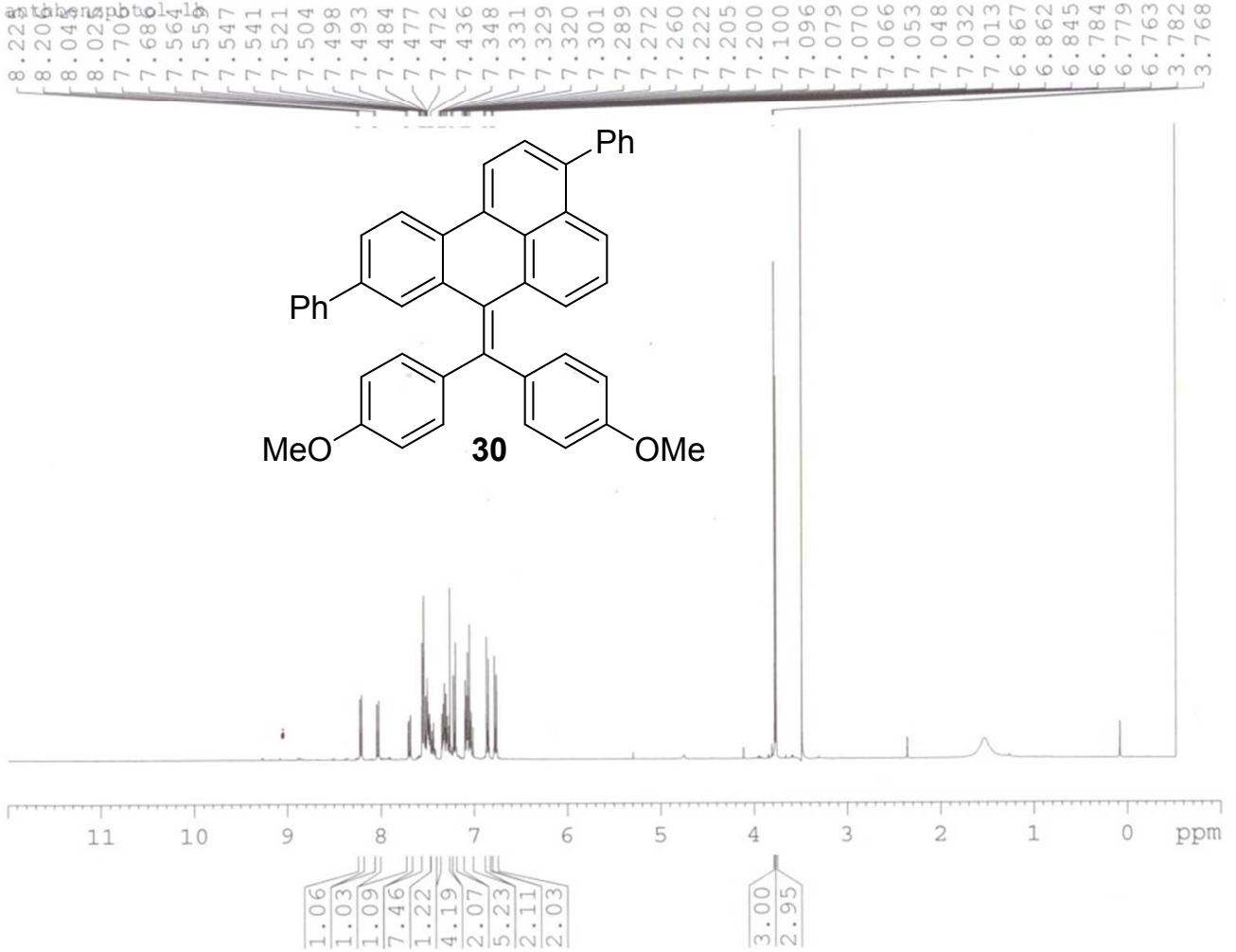

Figure S42. ${ }^{1} \mathrm{H}$ NMR spectrum of 7-(bis(4-methoxyphenyl)methylene)-3,9-diphenyl-7Hbenzo $[d e]$ anthracene (30) 
Supporting Information

anthbenzphtol suz c13

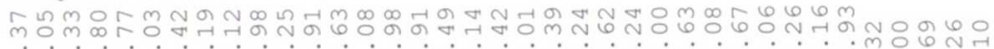

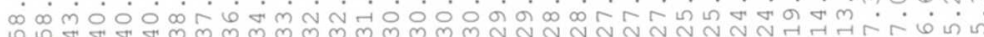

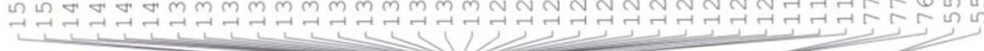
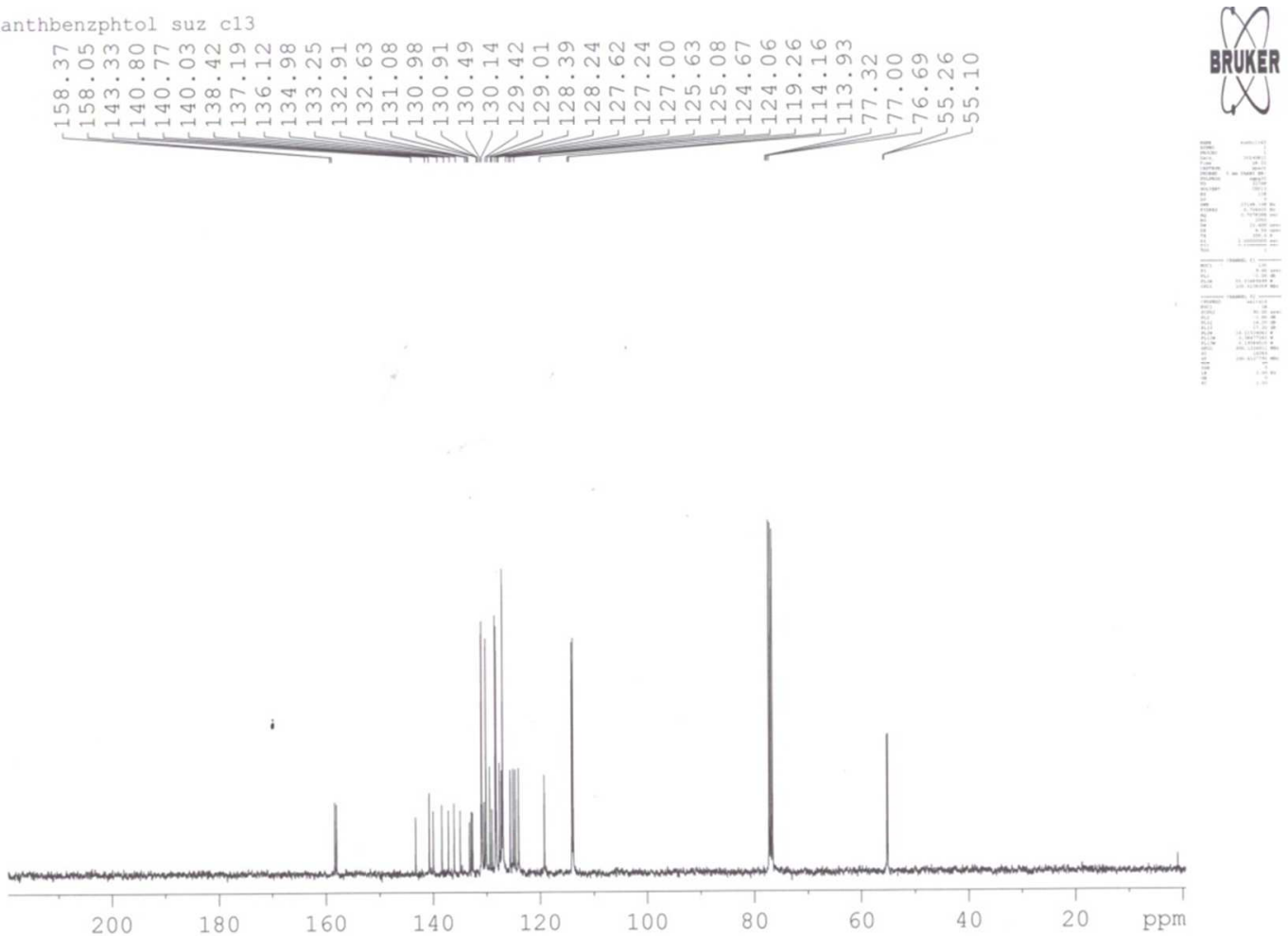

Figure S43. ${ }^{13} \mathrm{C}$ NMR spectrum of 7-(bis(4-methoxyphenyl)methylene)-3,9-diphenyl-

$7 H$-benzo[de]anthracene (30)
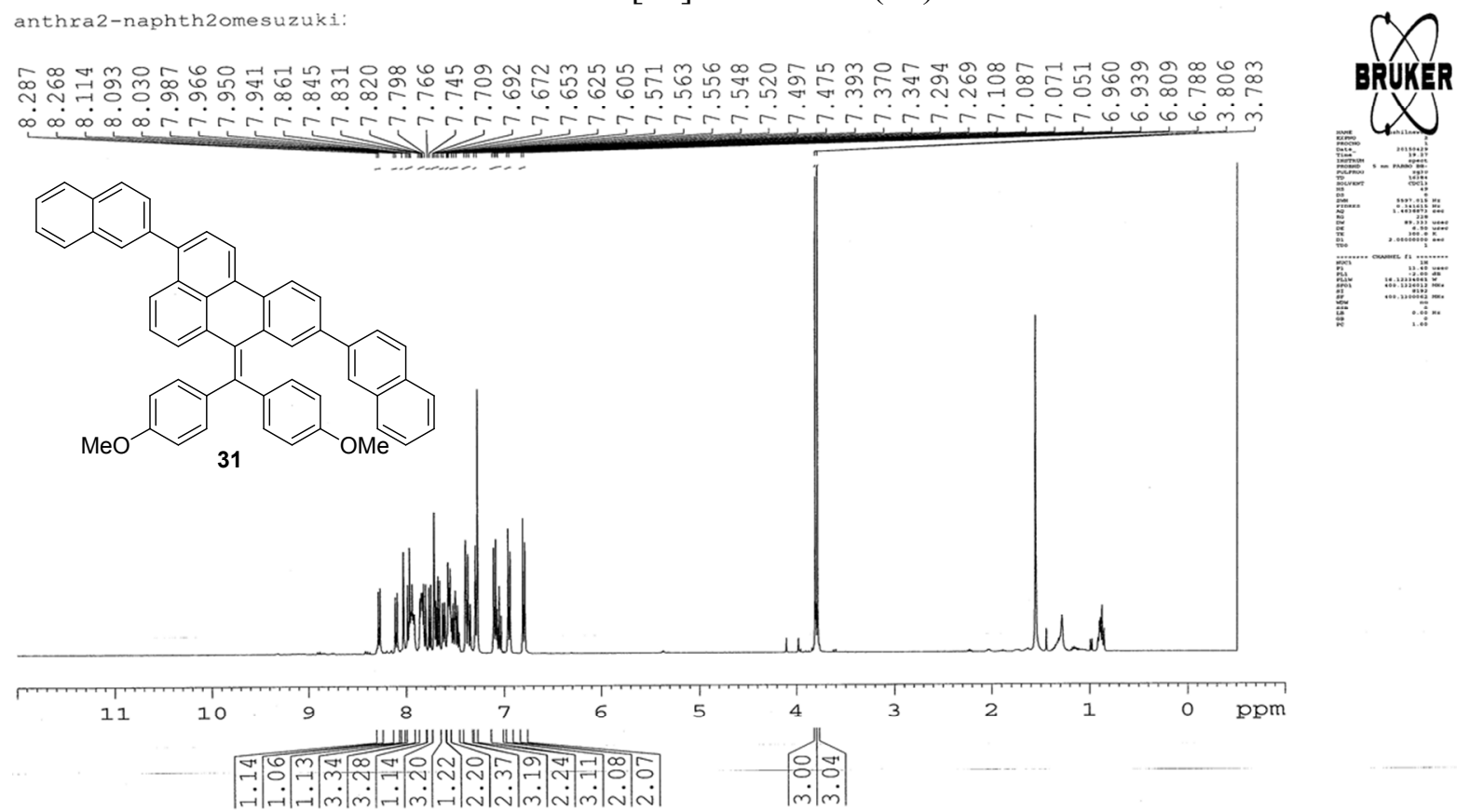

Figure S44. ${ }^{1}$ NMR spectrum of 7-(dichloromethylene)-3,9-di(naphthalen-2-yl)-7Hbenzo[de]anthracene (31) 
Supporting Information

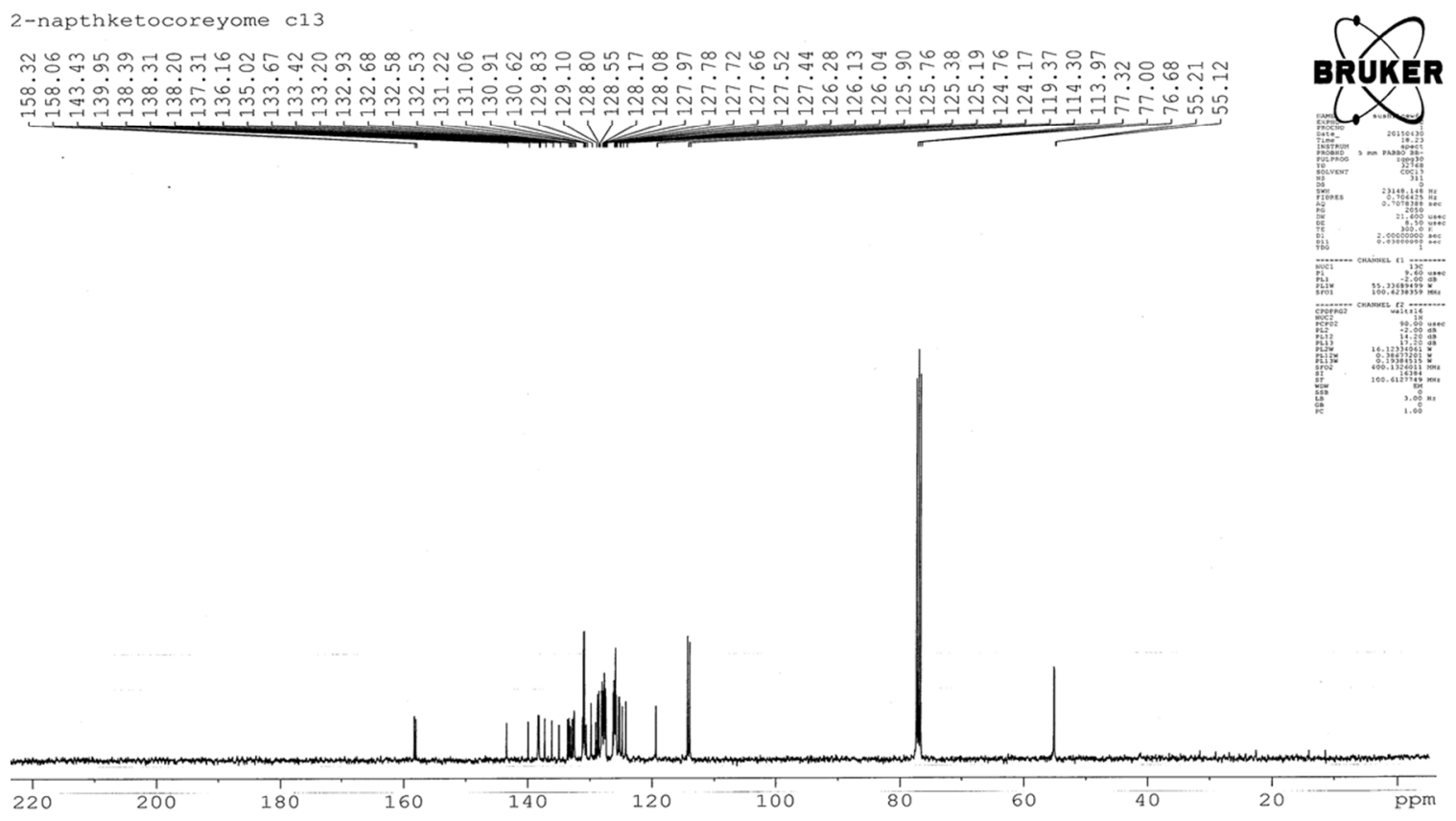

Figure S45. ${ }^{13} \mathrm{C}$ NMR spectrum of 7-(dichloromethylene)-3,9-di(naphthalen-2-yl)-7Hbenzo[de]anthracene (31)

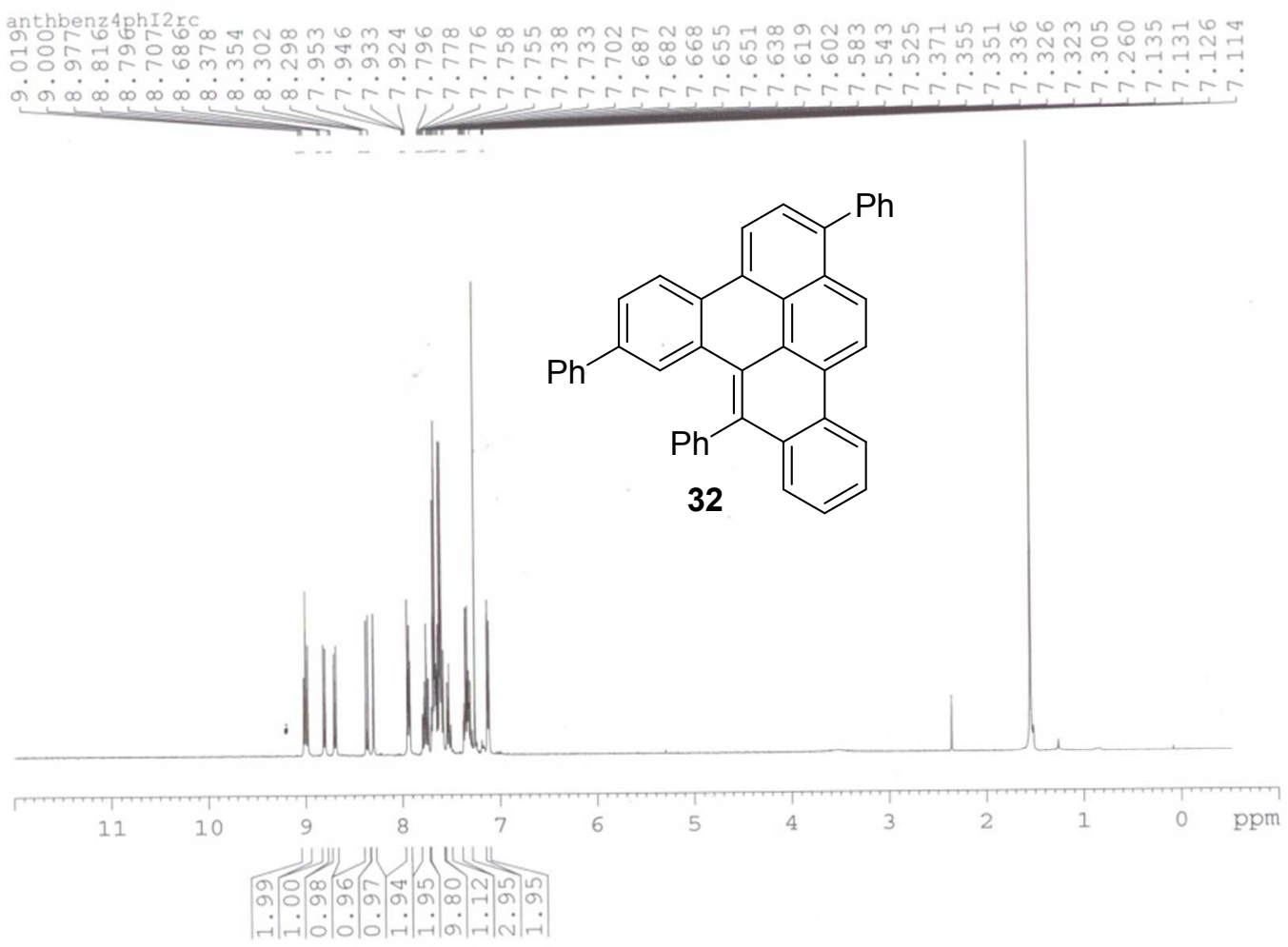

Figure S46. ${ }^{1}$ H NMR spectrum of 1,6,8-triphenyldibenzo[f,pqr]tetraphene (32) 
Supporting Information
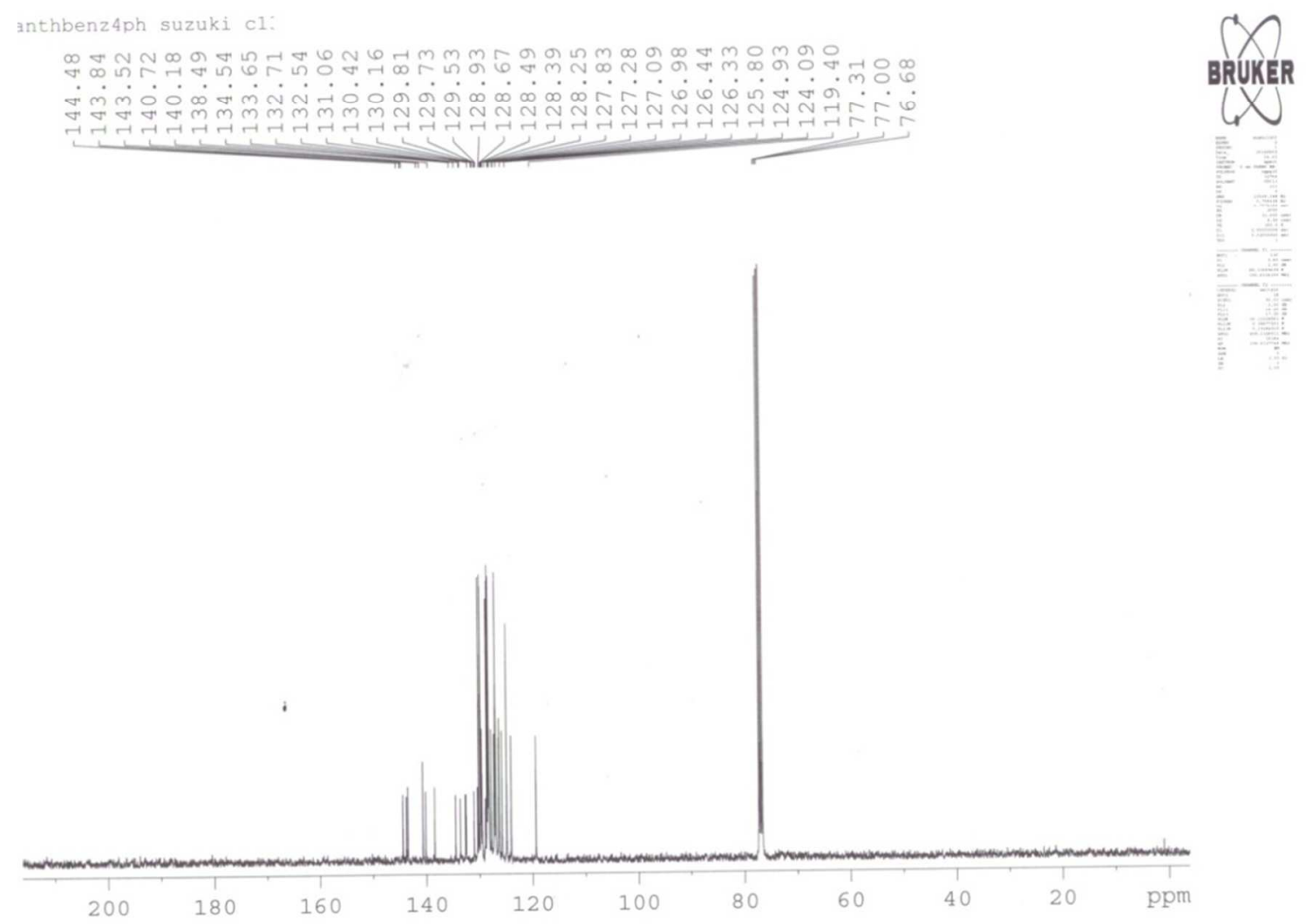

Figure S47. ${ }^{13} \mathrm{C}$ NMR spectrum of 1,6,8-triphenyldibenzo[f,pqr $]$ tetraphene (32)

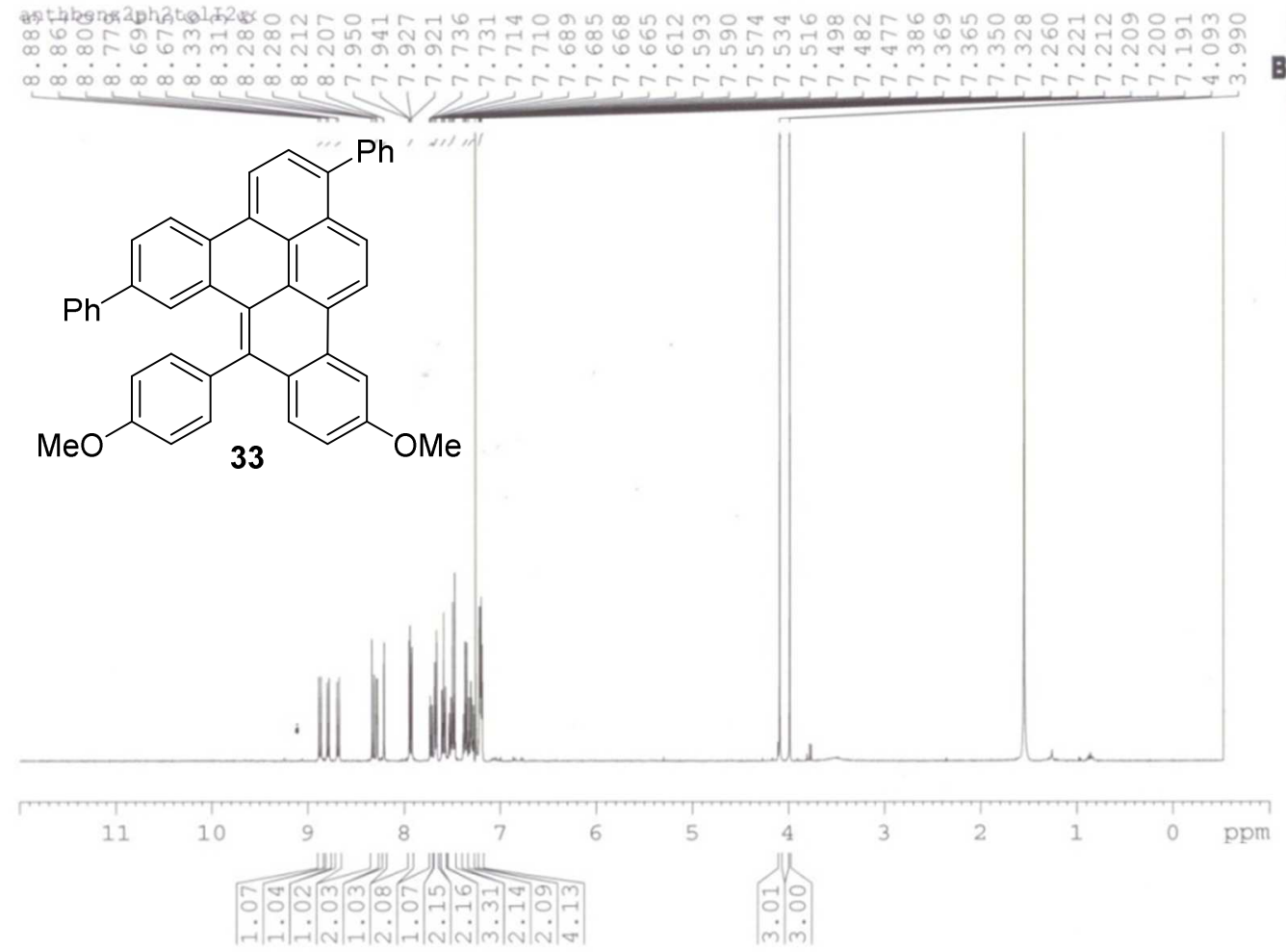

Figure S48. ${ }^{1} \mathrm{H}$ NMR spectrum of 311-methoxy-8-(4-methoxyphenyl)-1,6diphenyldibenzo[f,pqr] tetraphene (33) 
Supporting Information

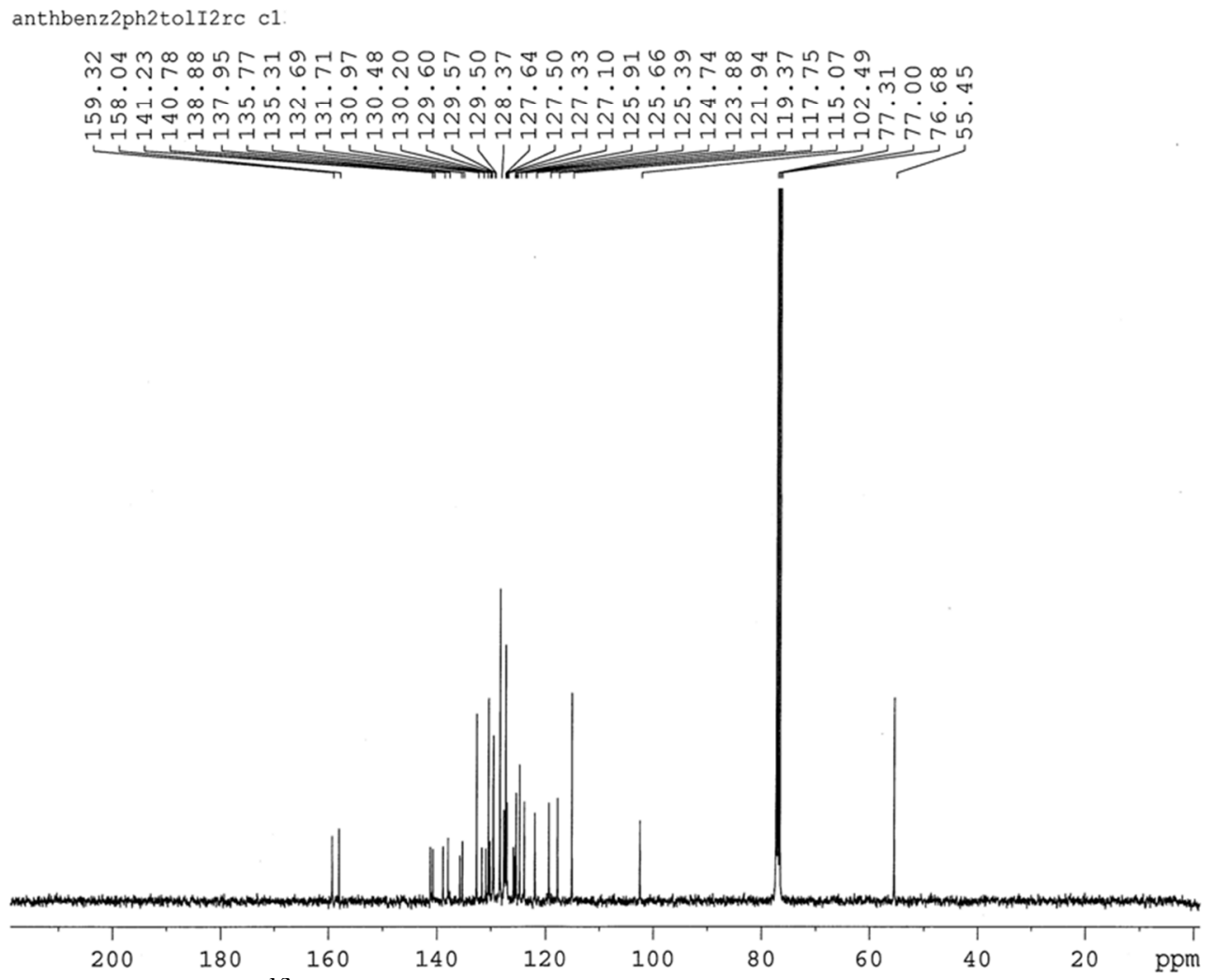

Figure S49. ${ }^{13} \mathrm{C}$ NMR spectrum of 311-methoxy-8-(4-methoxyphenyl)-1,6diphenyldibenzo[f,pqr] tetraphene (33)

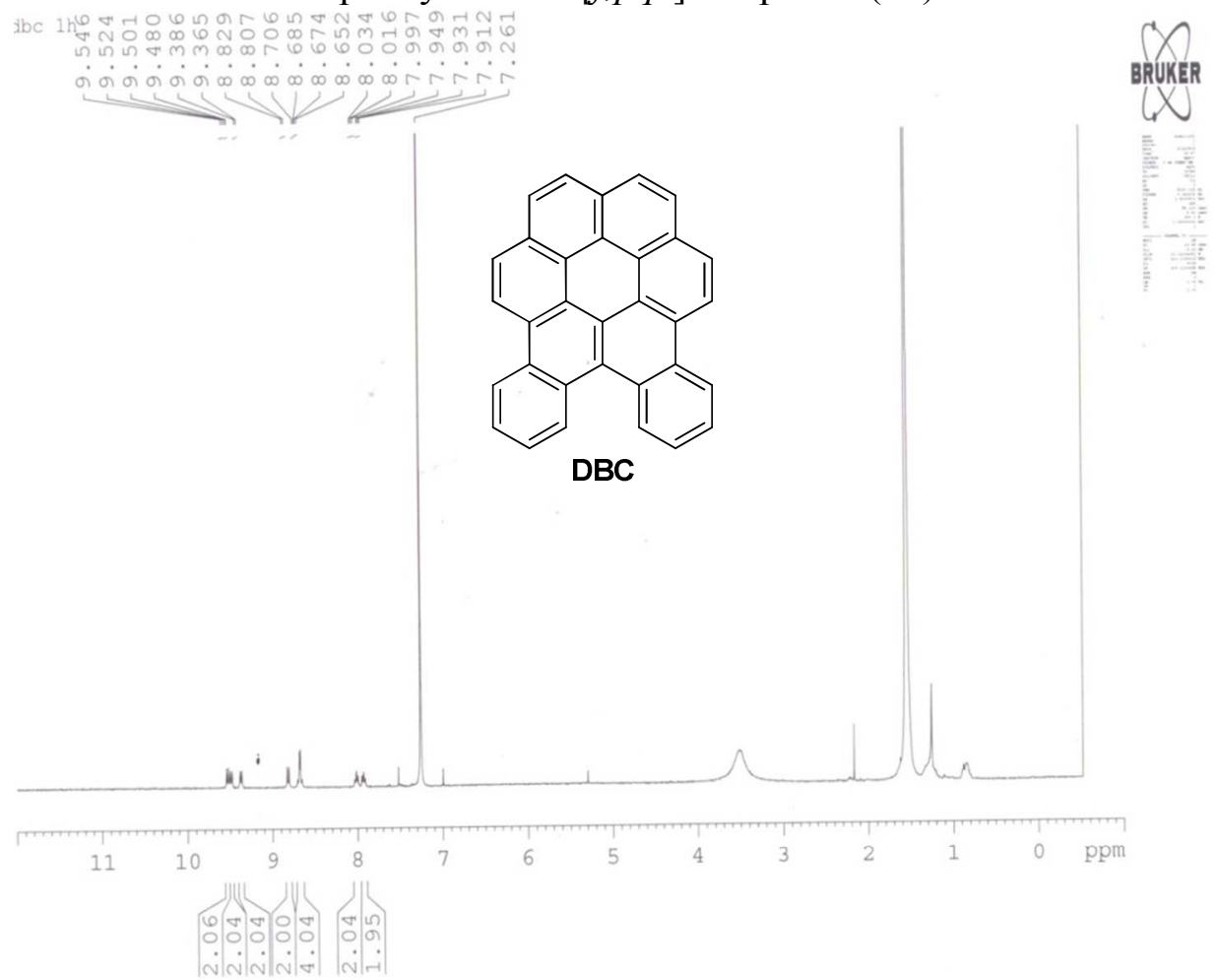

Figure S50. ${ }^{1} \mathrm{H}$ NMR spectrum of dibenzo $[a, d]$ coronene (DBC) 
Supporting Information

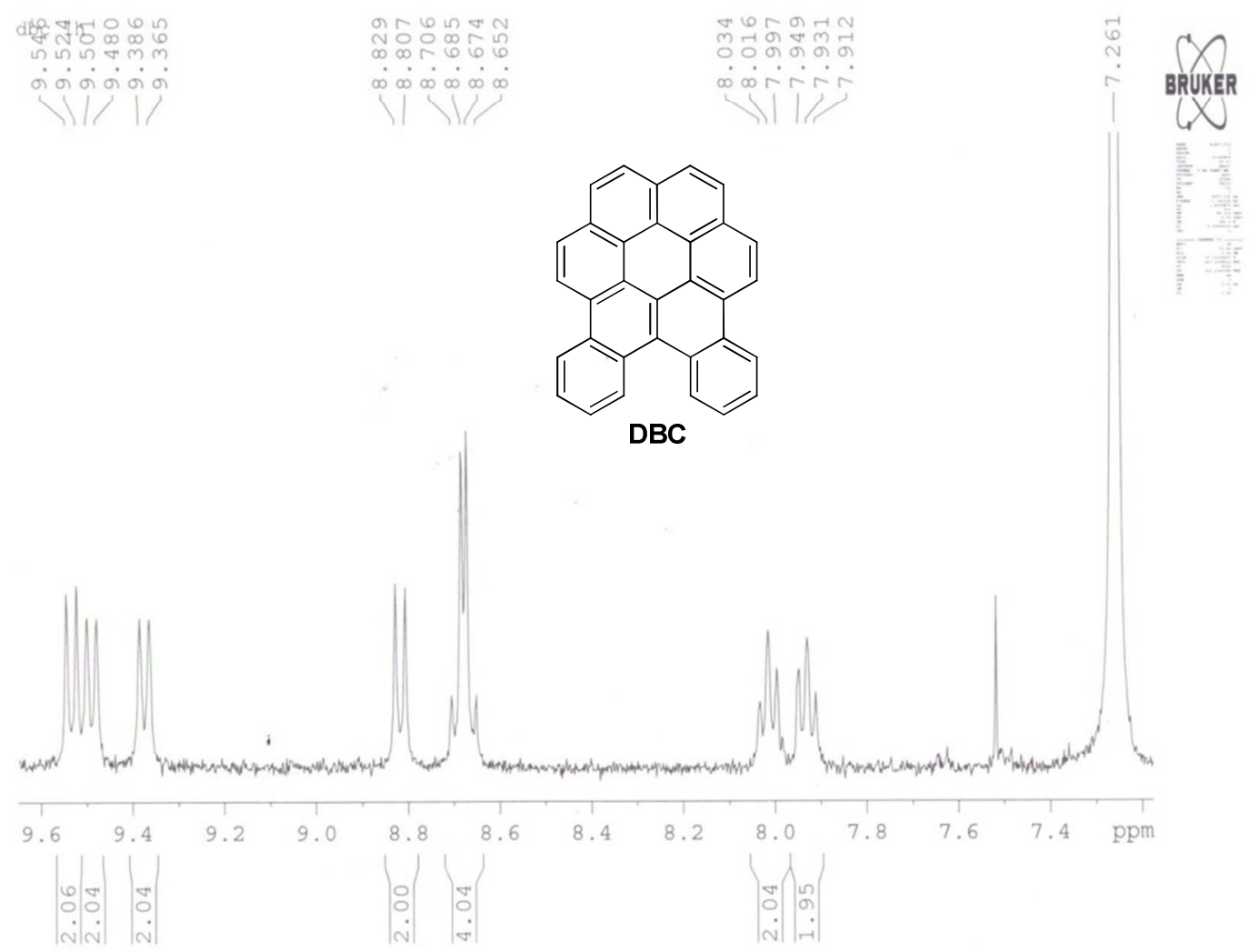

Figure S51. ${ }^{1} \mathrm{H}$ NMR spectrum (expansion) of dibenzo[a,d]coronene (DBC)

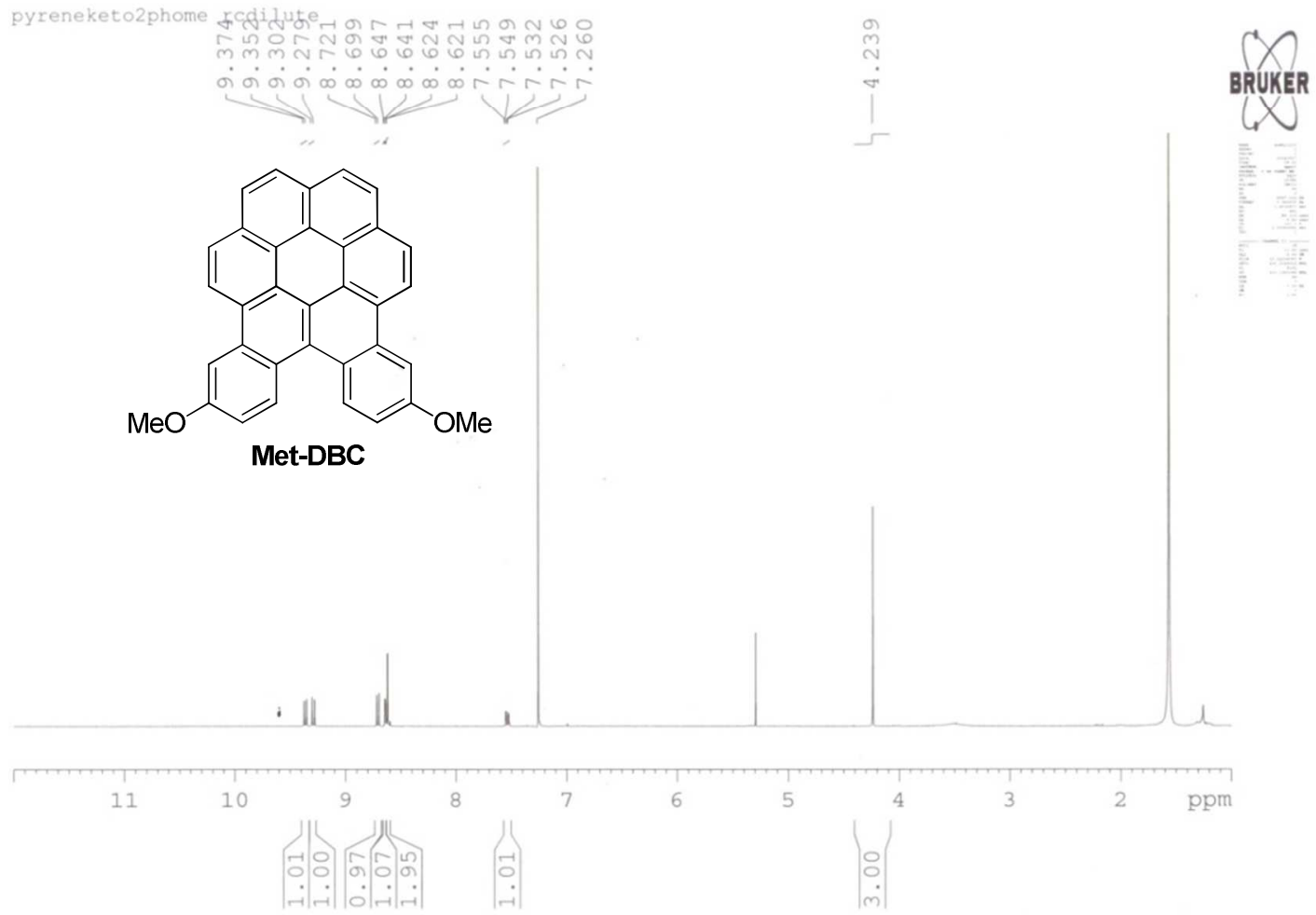

Figure S52. ${ }^{1} \mathrm{H}$ NMR spectrum of 8,13-dimethoxydibenzo[a,d]coronene (Met-DBC) 


\section{Supporting Information}

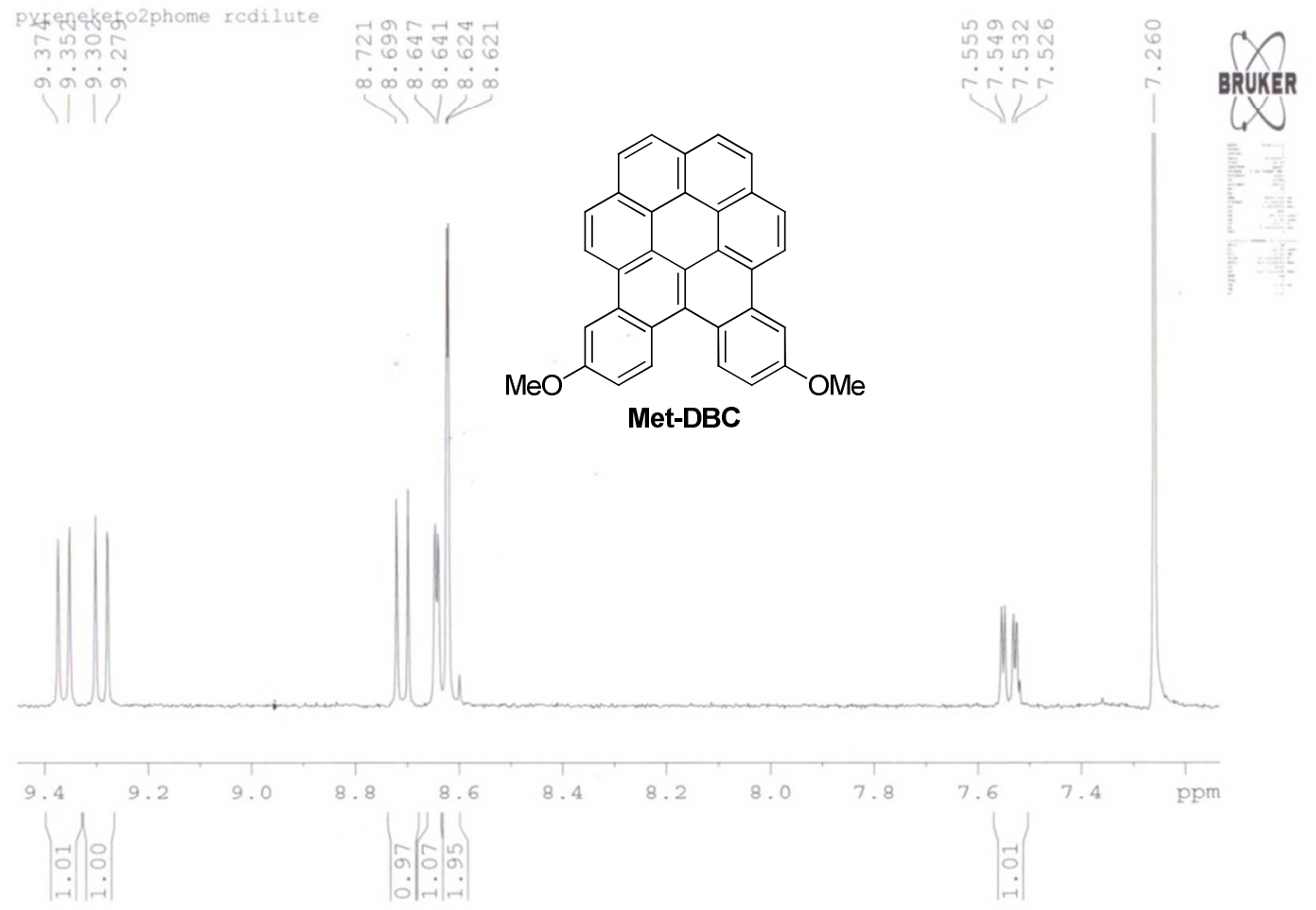

Figure S53. ${ }^{1} \mathrm{H}$ NMR spectrum (expansion) of 8,13-dimethoxydibenzo[a,d]coronene (Met-DBC)

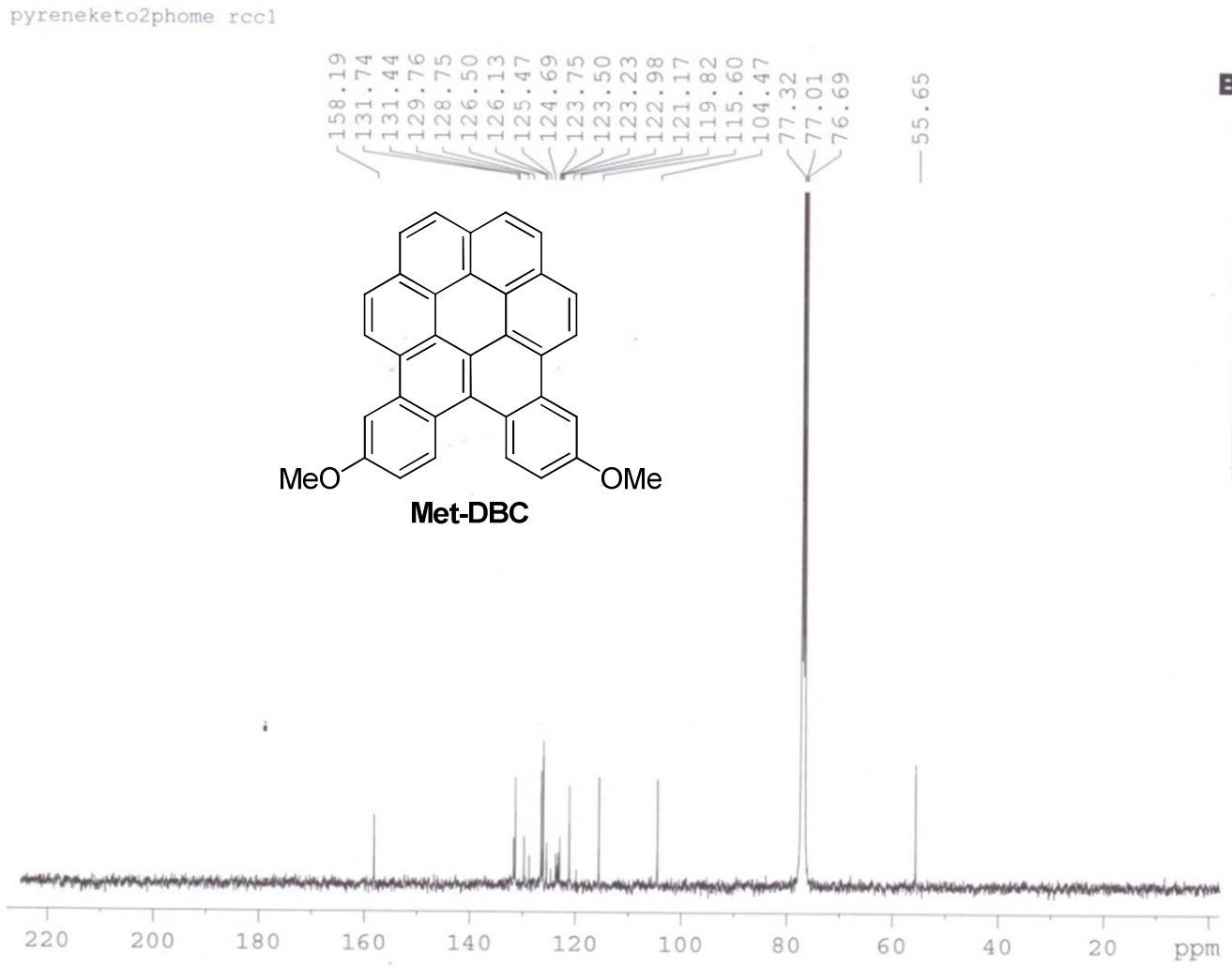

Figure S54. ${ }^{13} \mathrm{C}$ NMR spectrum of 8,13-dimethoxydibenzo[ $[a, d]$ coronene (Met-DBC) 
Supporting Information

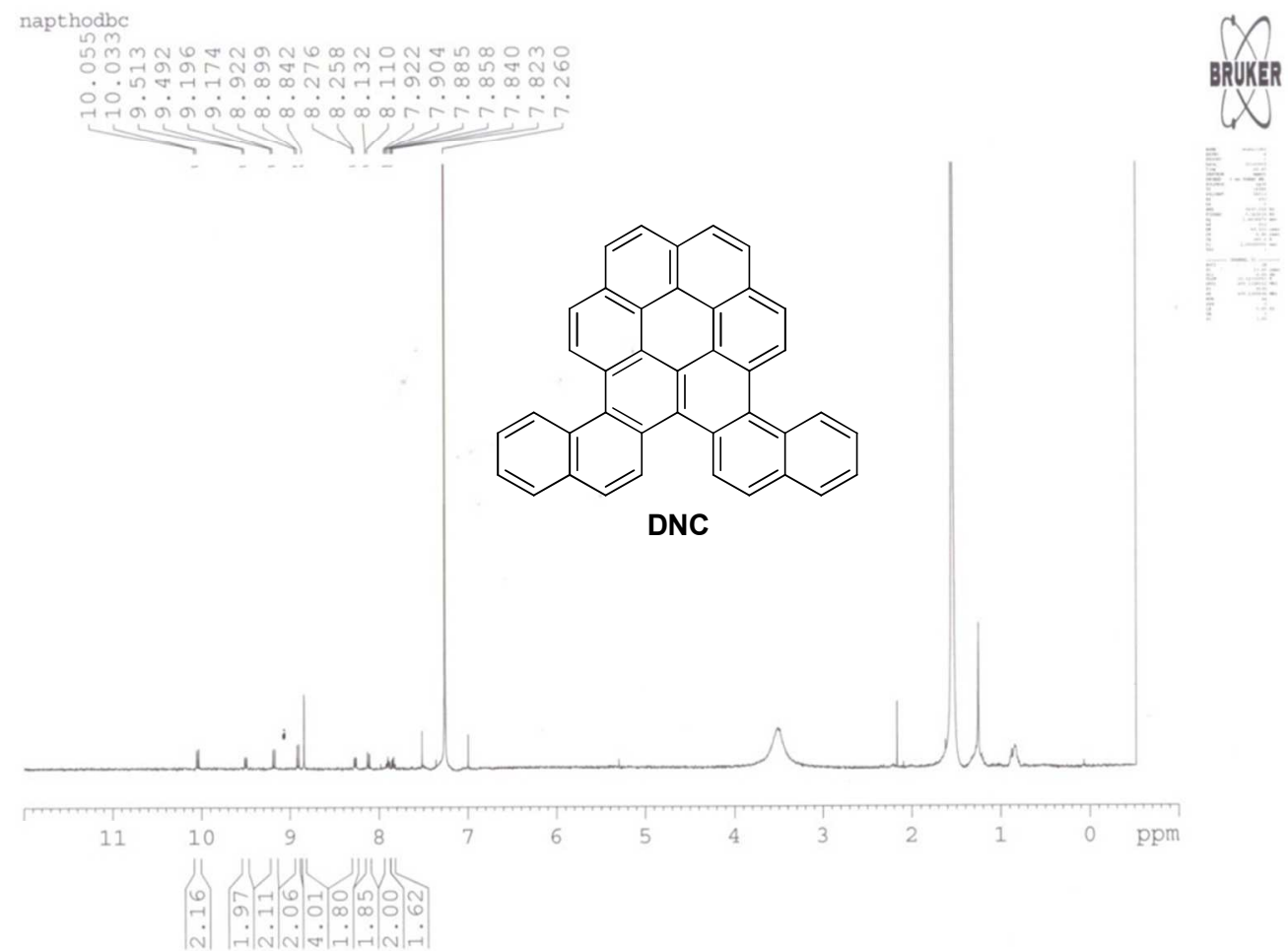

Figure S55. ${ }^{1} \mathrm{H}$ NMR spectrum of dinaphtho[1,2-a:2',1'-d]coronene (DNC)

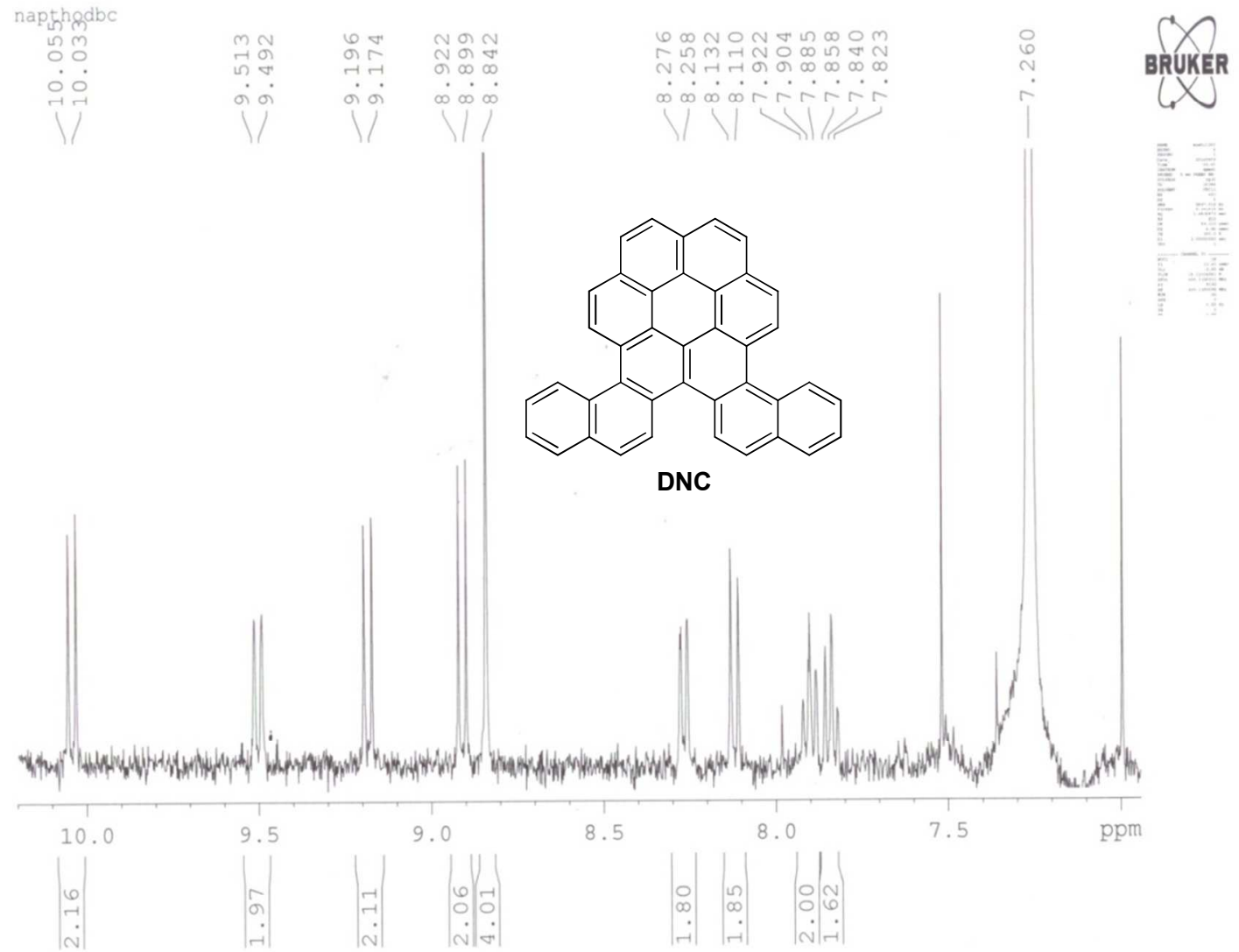

Figure S56. ${ }^{1} \mathrm{H}$ NMR spectrum (expansion) of dinaphtho[1,2-a:2',1'-d]coronene (DNC) 


\section{Supporting Information}

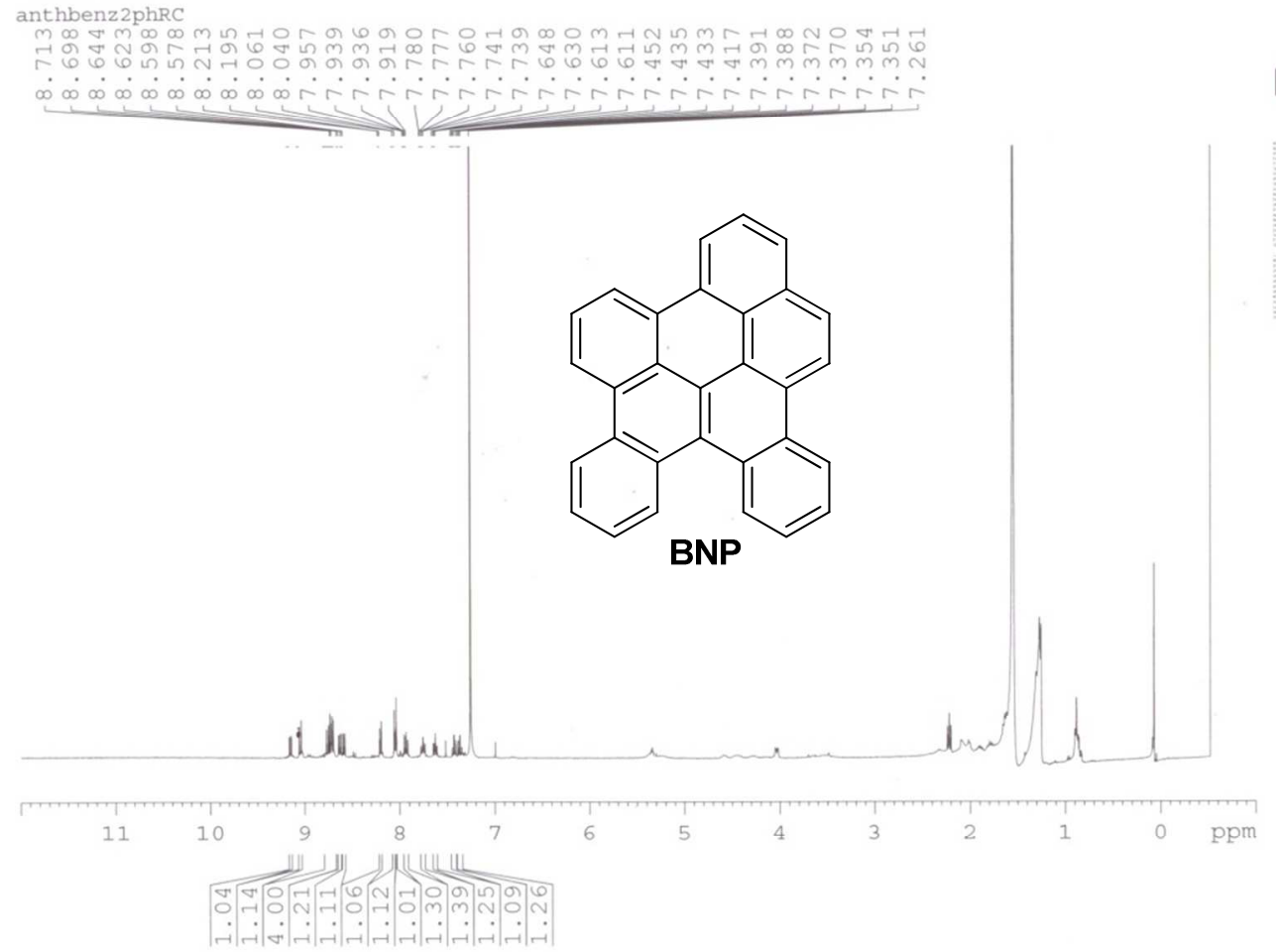

Figure S57. ${ }^{1} \mathrm{H}$ NMR spectrum of benzo[b]naphtho[1,2,3,4-pqr]perylene (BNP)

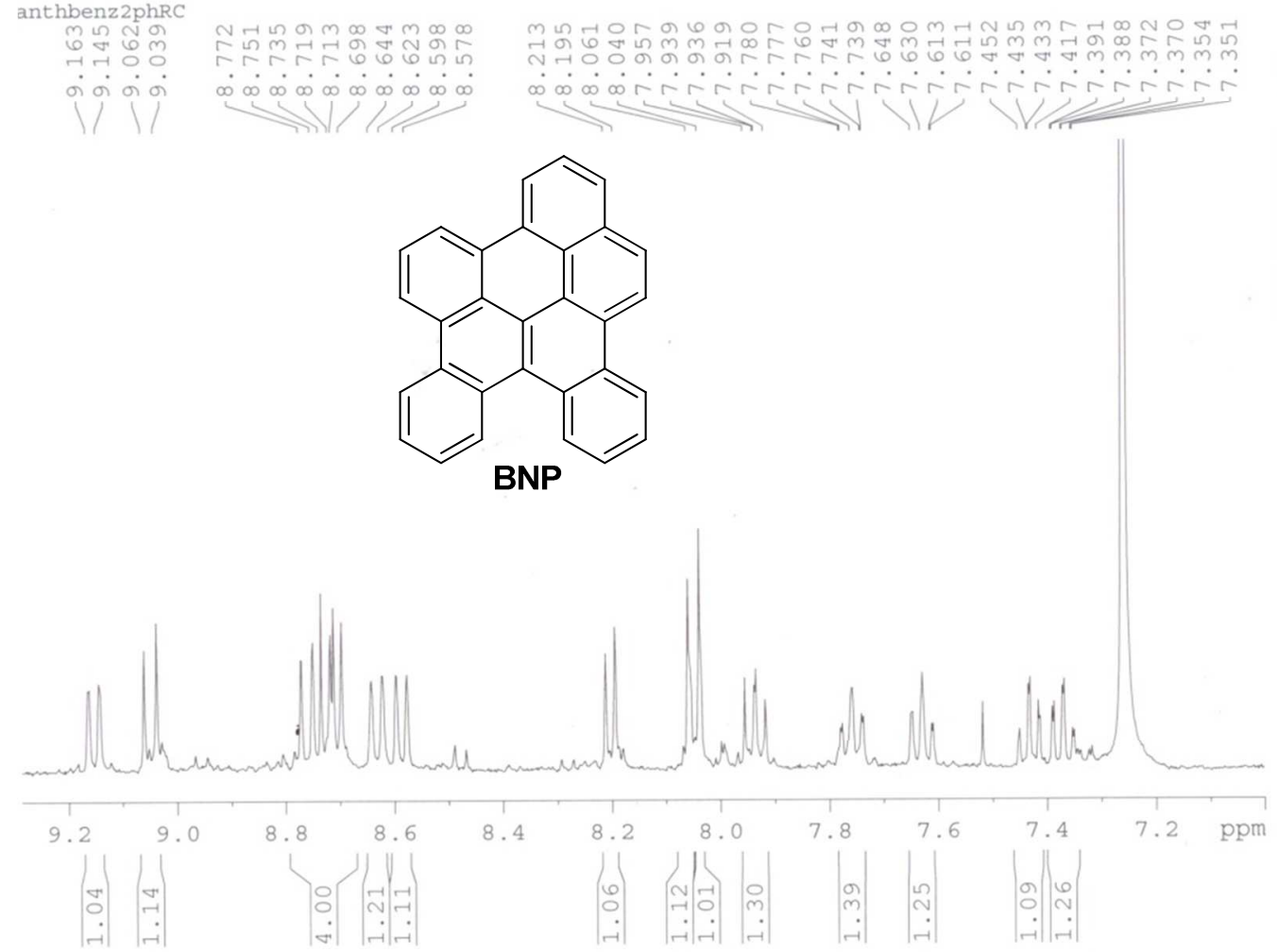

Figure S58. ${ }^{1} \mathrm{H}$ NMR spectrum (expansion) of benzo[b]naphtho[1,2,3,4-pqr]perylene (BNP) 


\section{Supporting Information}

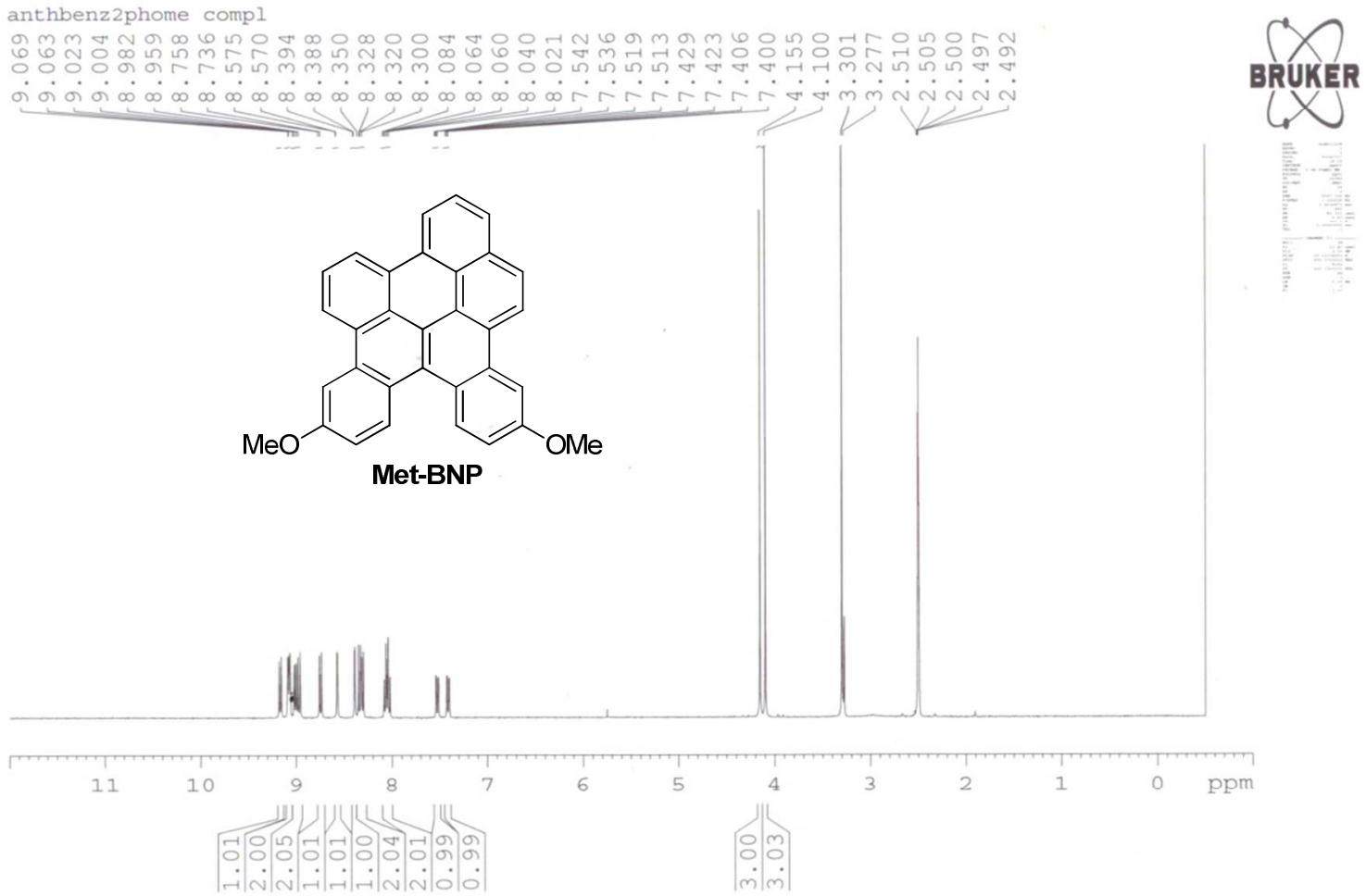

Figure S59. ${ }^{1} \mathrm{H}$ NMR spectrum of 2,7-dimethoxybenzo[b]naphtho[1,2,3,4-pqr]perylene (Met-BNP)

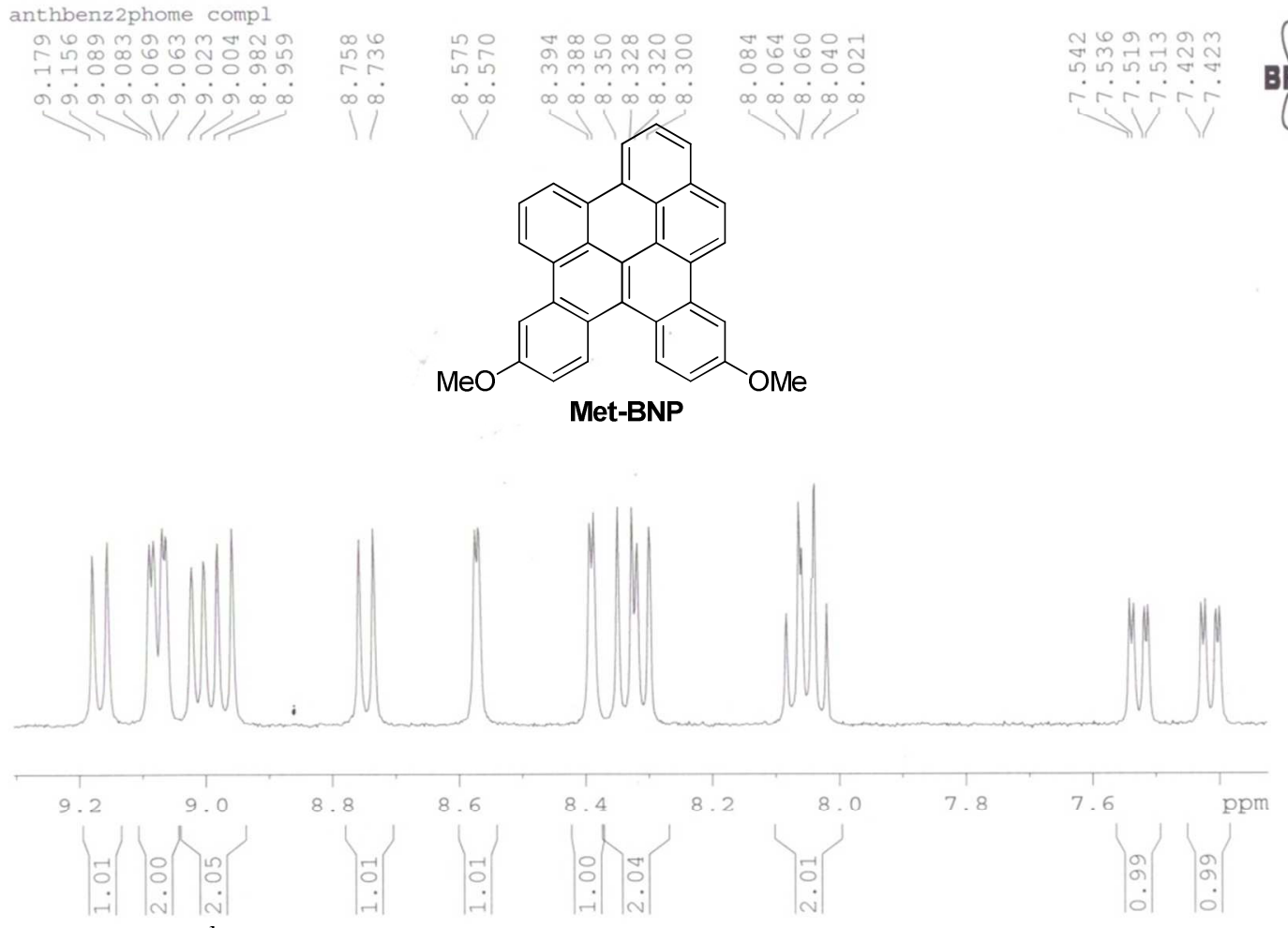

Figure S60. ${ }^{1} \mathrm{H}$ NMR spectrum (expansion) of 2,7-dimethoxybenzo[b]naphtho[1,2,3,4pqr]perylene (Met-BNP) 


\section{Supporting Information}

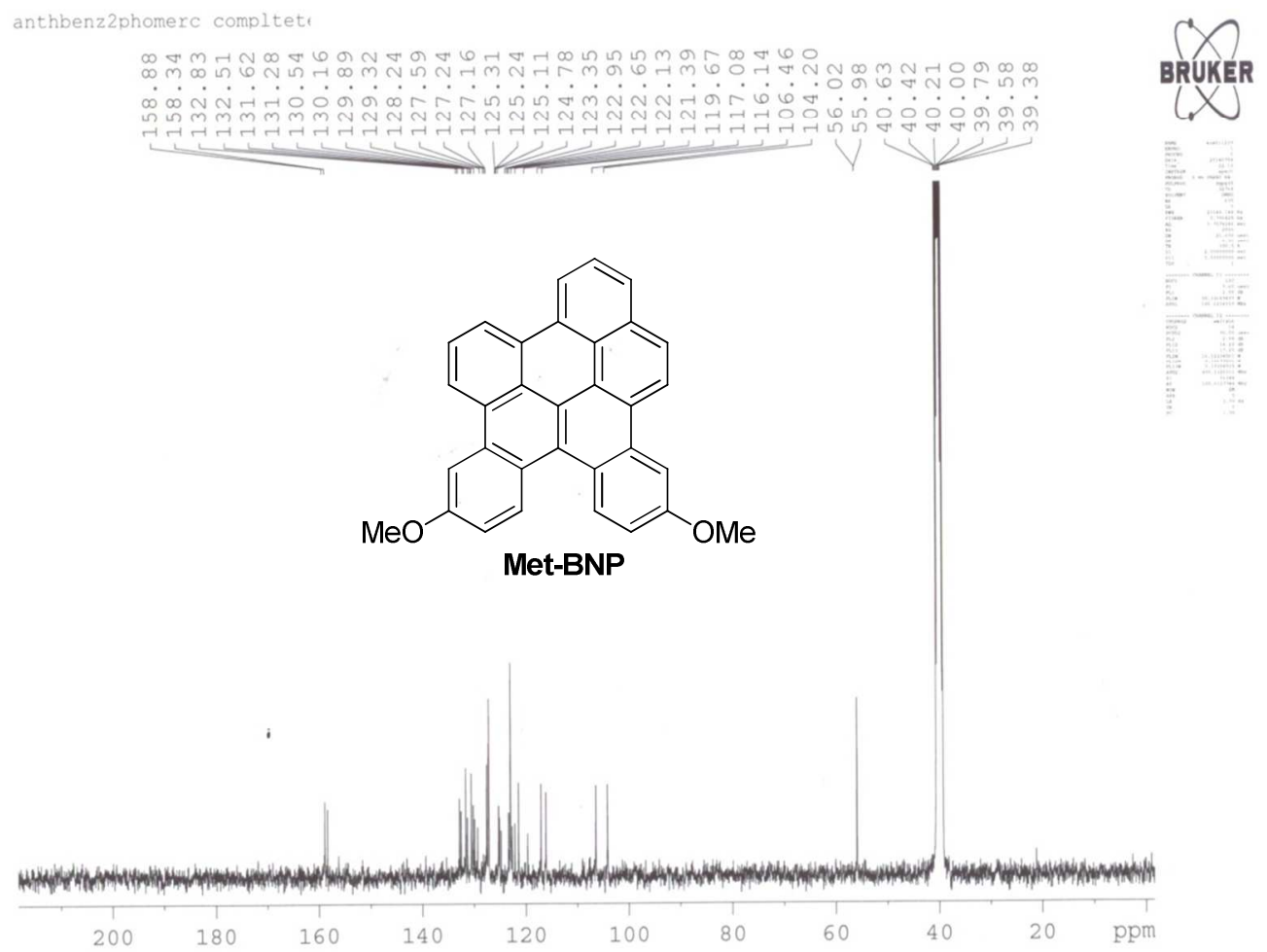

Figure S61. ${ }^{1 / 3} \mathrm{C}$ NMR spectrum of 2,7-dimethoxybenzo[b]naphtho[1,2,3,4-pqr]perylene (Met-BNP)

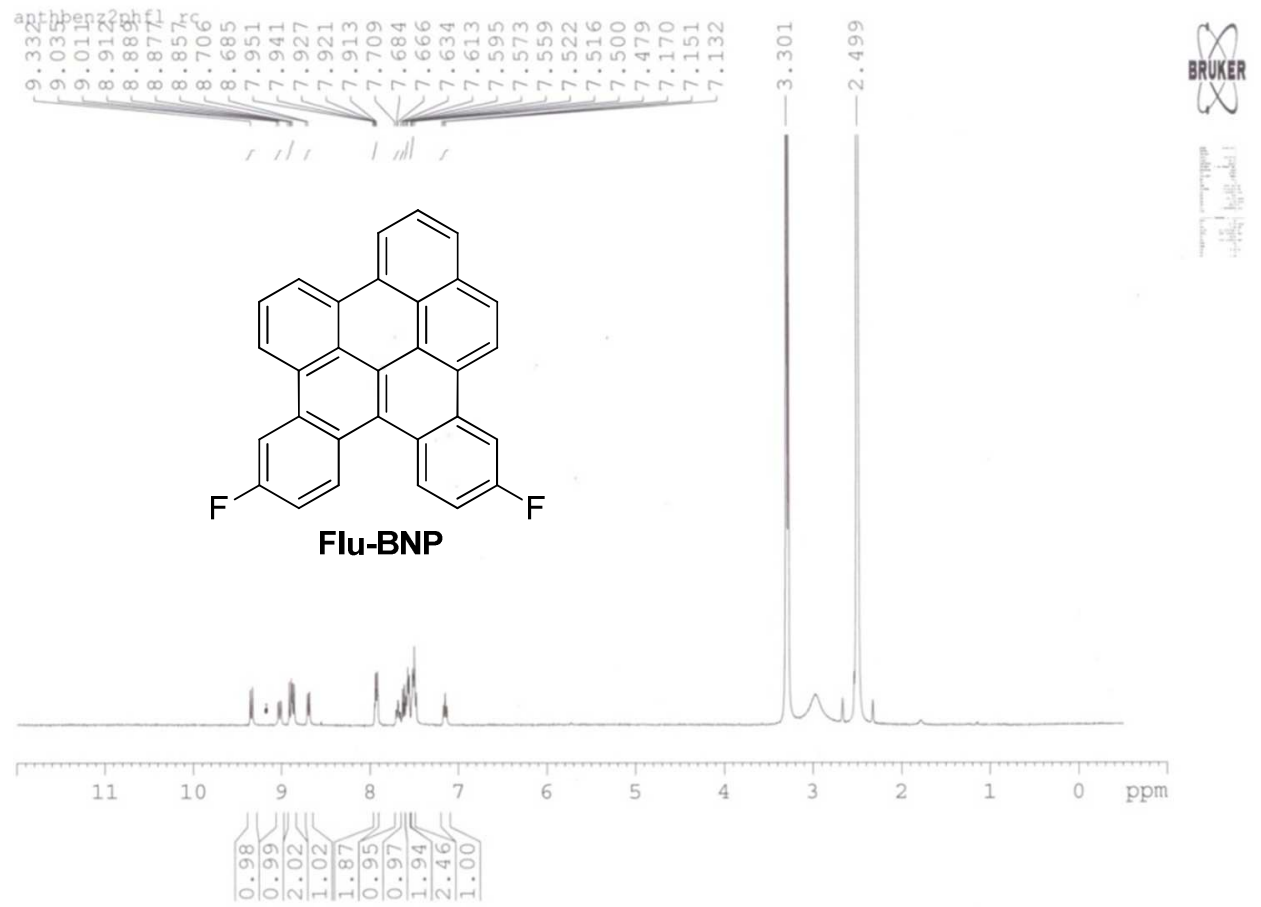

Figure S62. ${ }^{1} \mathrm{H}$ NMR spectrum of 2,7-difluorobenzo[b]naphtho[1,2,3,4-pqr]perylene (Flu-BNP) 
Supporting Information

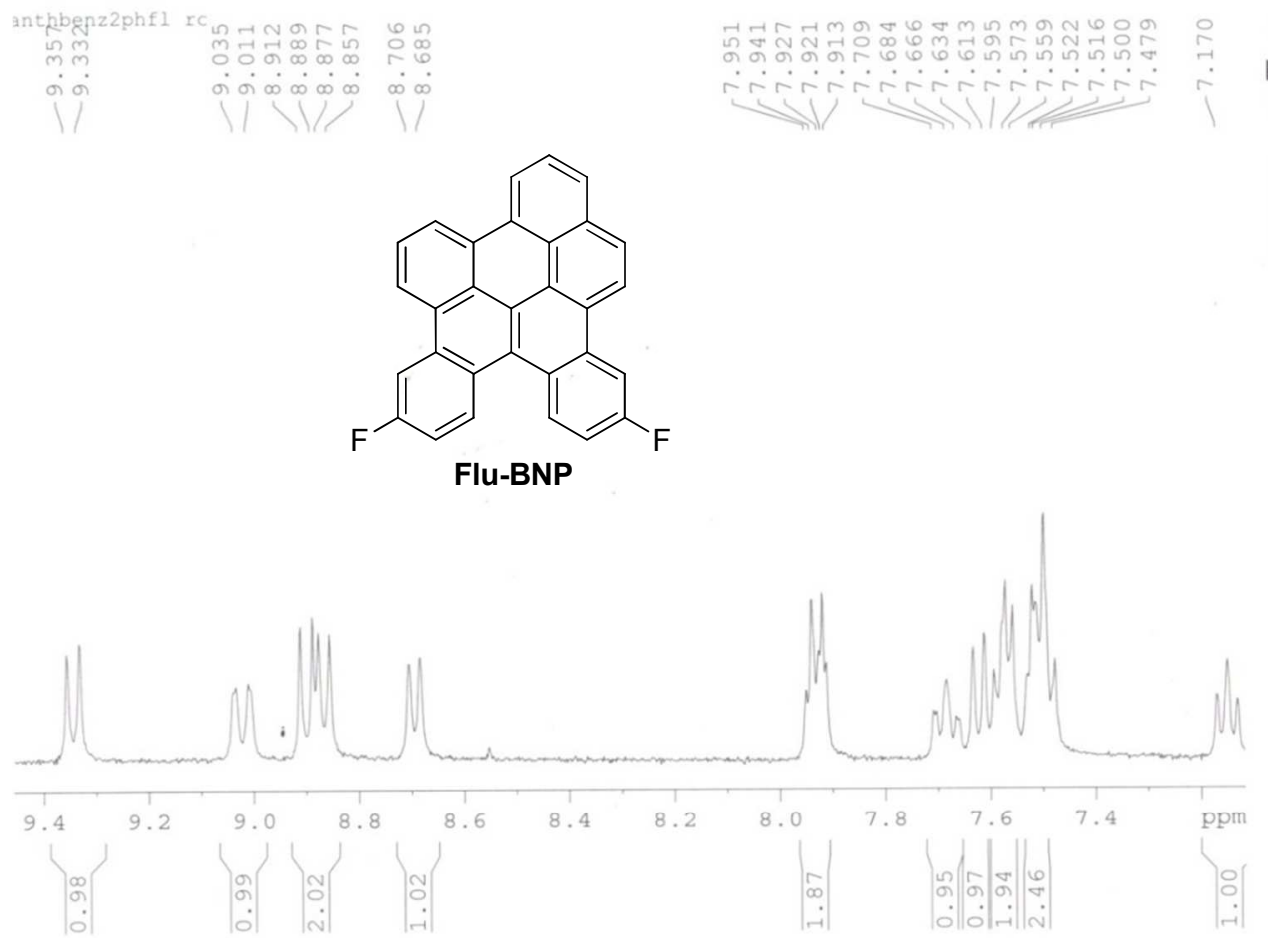

Figure S63. ' H NMR spectrum (expansion) of 2,7-difluorobenzo[b]naphtho[1,2,3,4pqr]perylene (Flu-BNP)

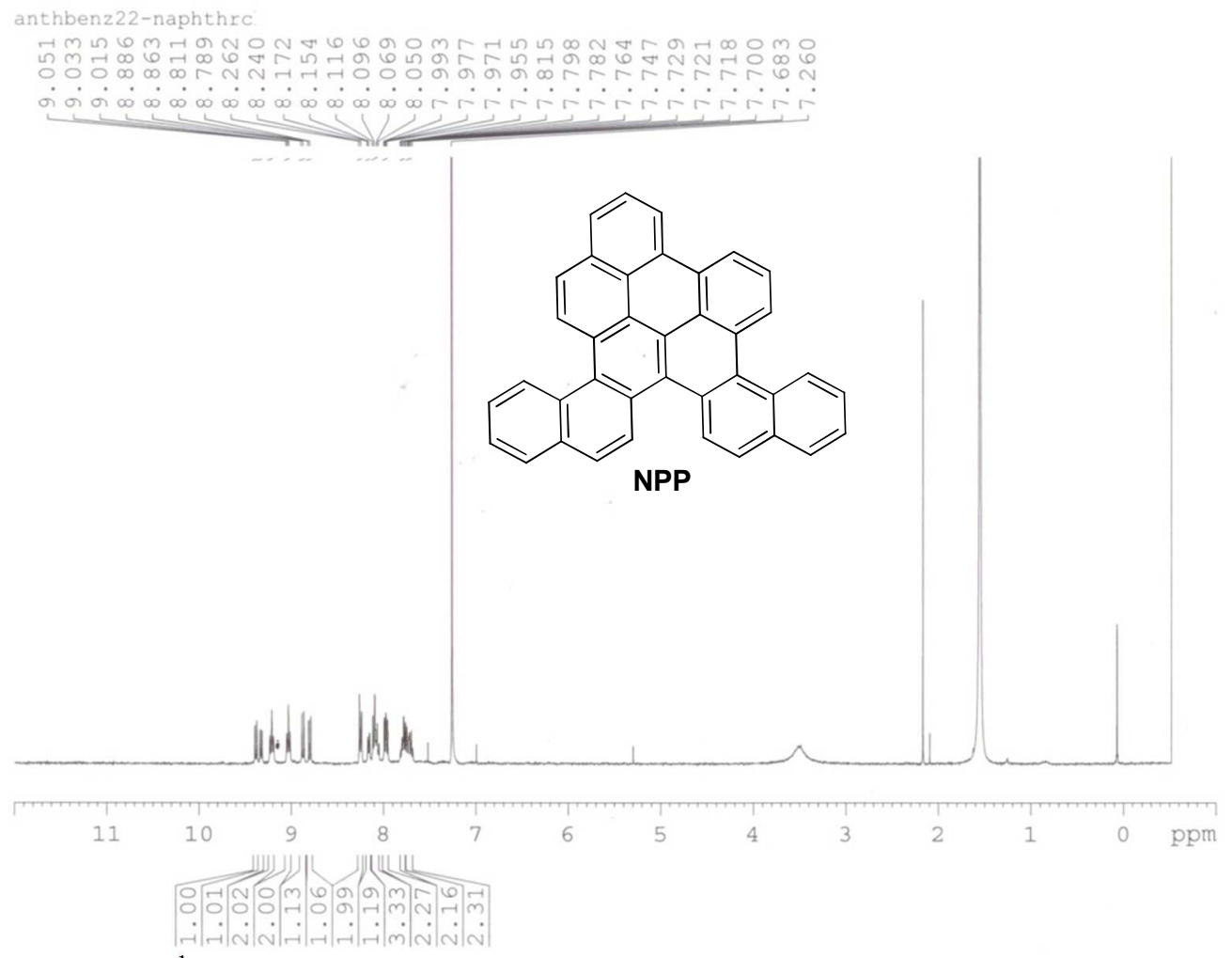

Figure S64. ${ }^{1} \mathrm{H}$ NMR spectrum of naphtho[2,1-b]phenanthro[4,3,2,1-pqr]perylene (NPP) 
Supporting Information
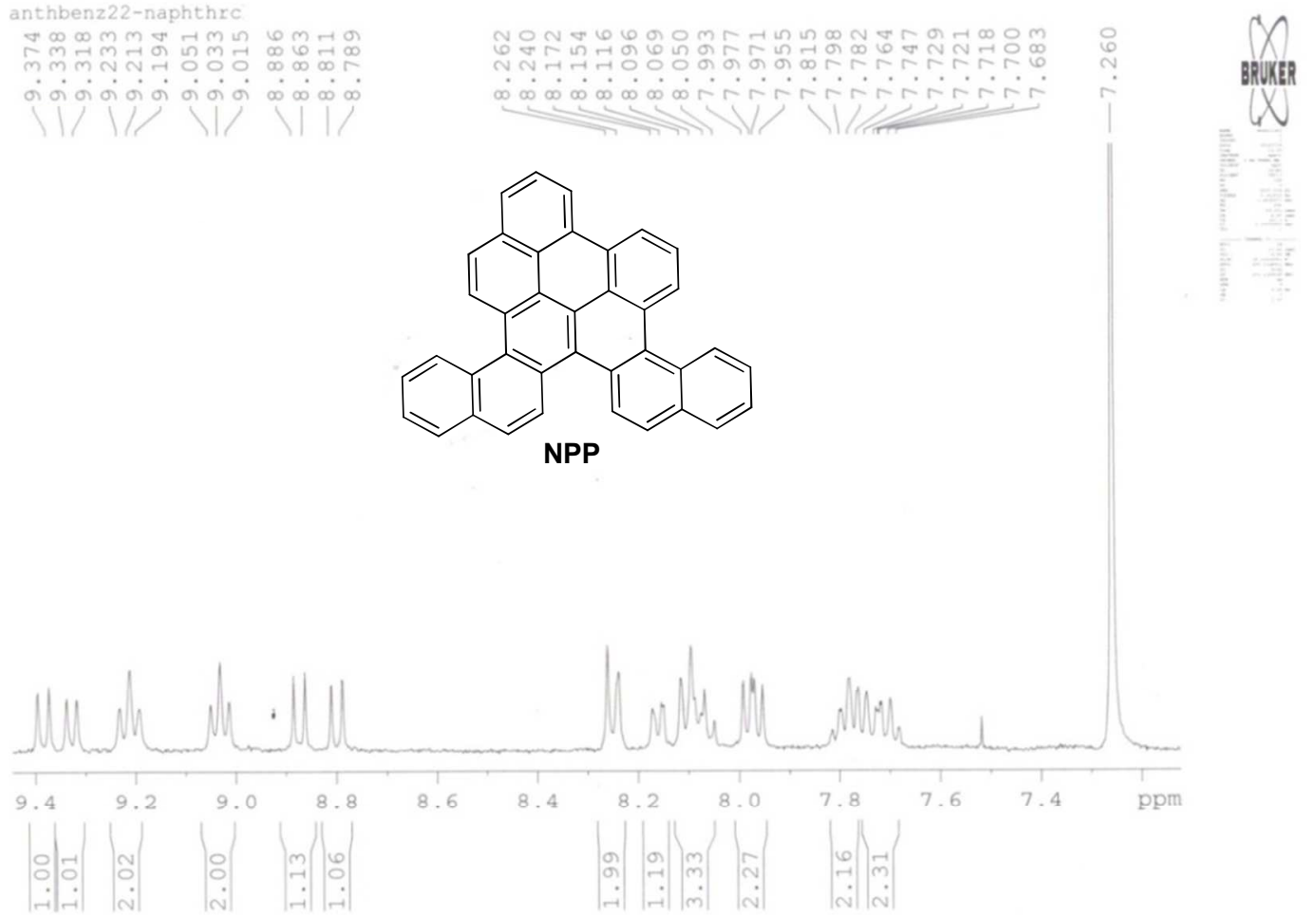

Figure S65. ${ }^{1} \mathrm{H}$ NMR spectrum (expansion) of naphtho[2,1-b]phenanthro[4,3,2,1pqr]perylene (NPP)

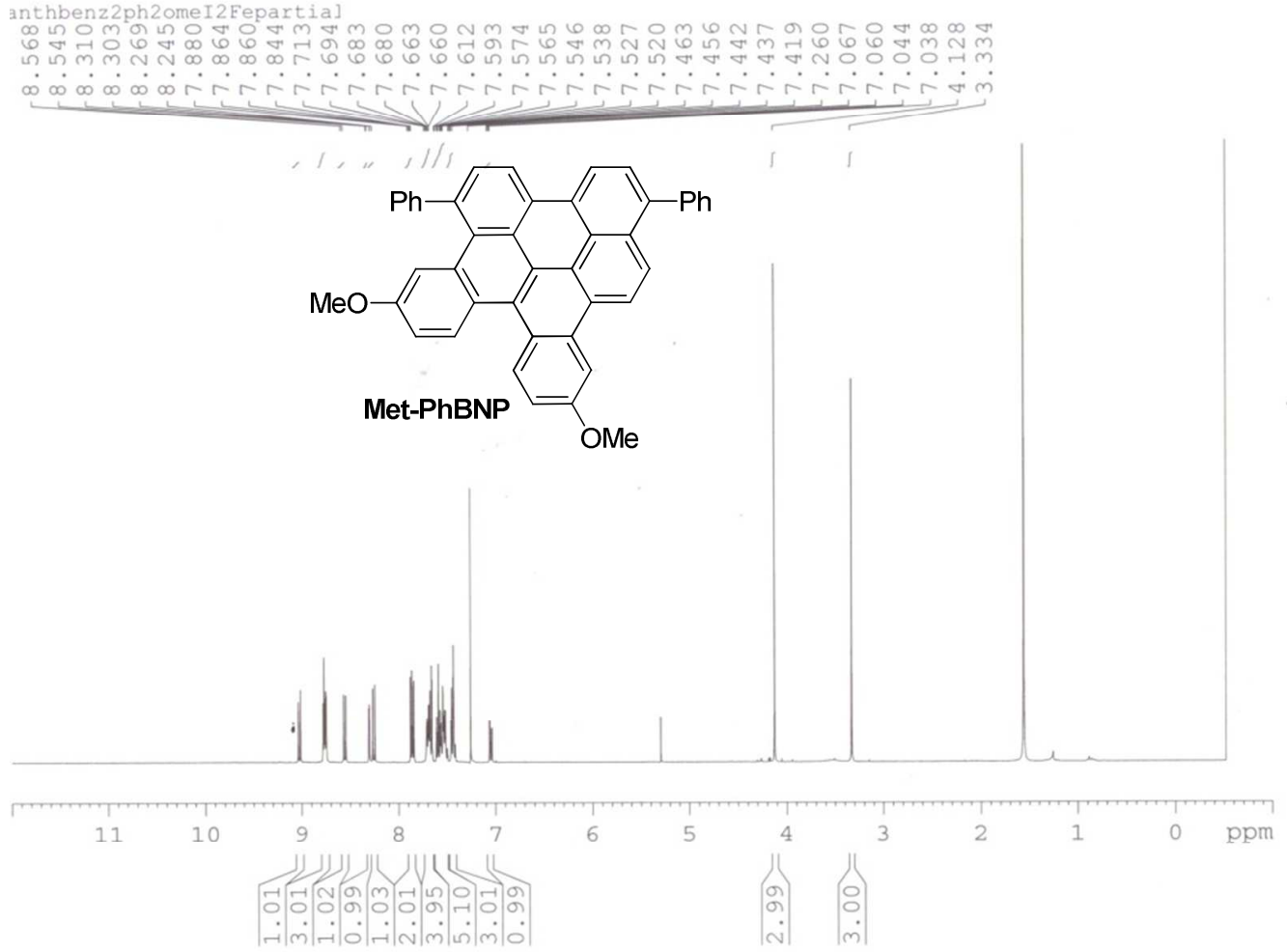

Figure S66. ${ }^{1} \mathrm{H}$ NMR spectrum of 2,7-dimethoxy-9,14-diphenylbenzo[b]naphtho[1,2,3,4pqr]perylene (Met-PhBNP) 
Supporting Information

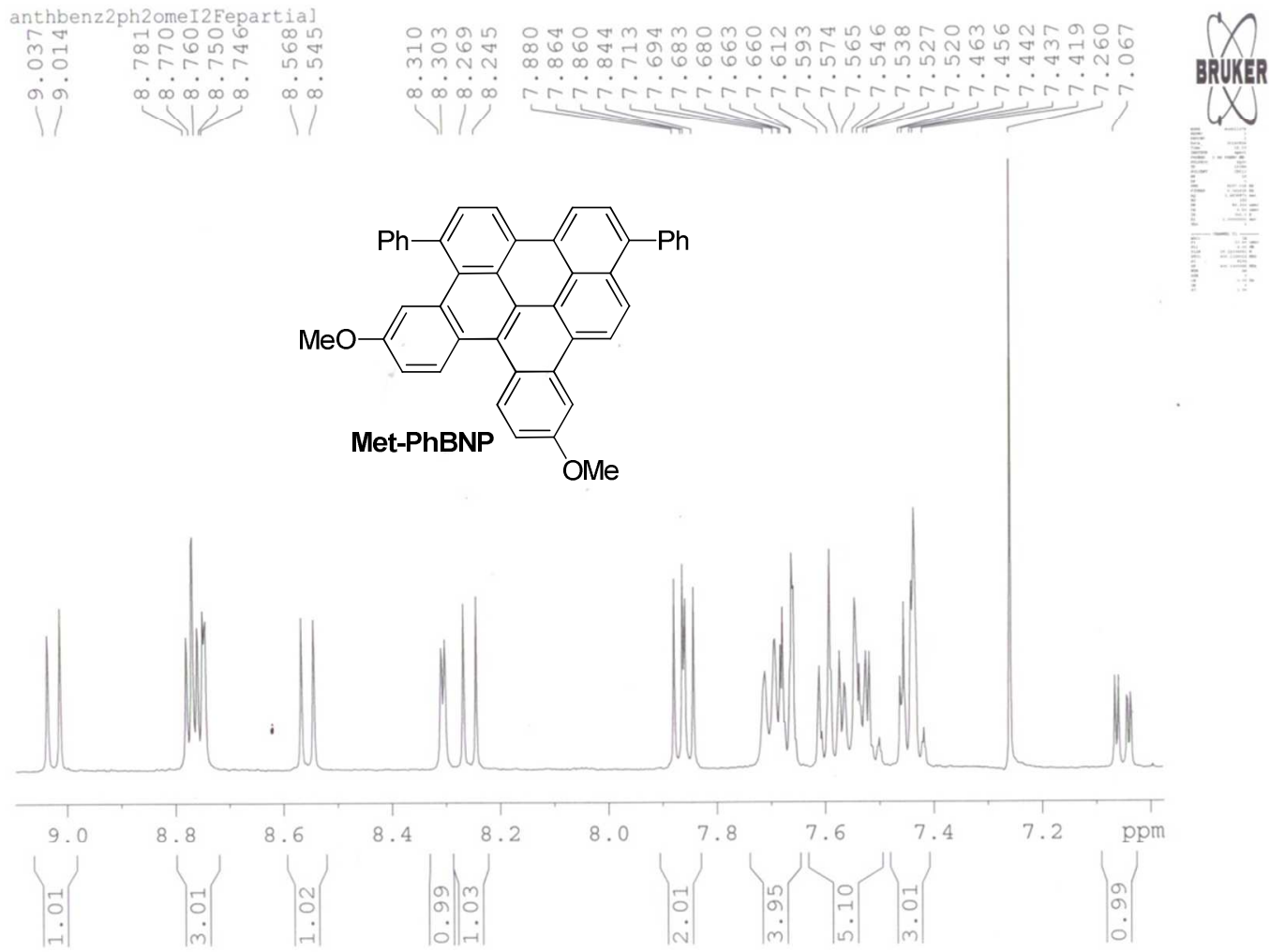

Figure S67. ${ }^{1} \mathrm{H}$ NMR spectrum (expansion) of 2,7-dimethoxy-9,14-diphenylbenzo $[b]$ naphtho[1,2,3,4-pqr] perylene (Met-PhBNP)

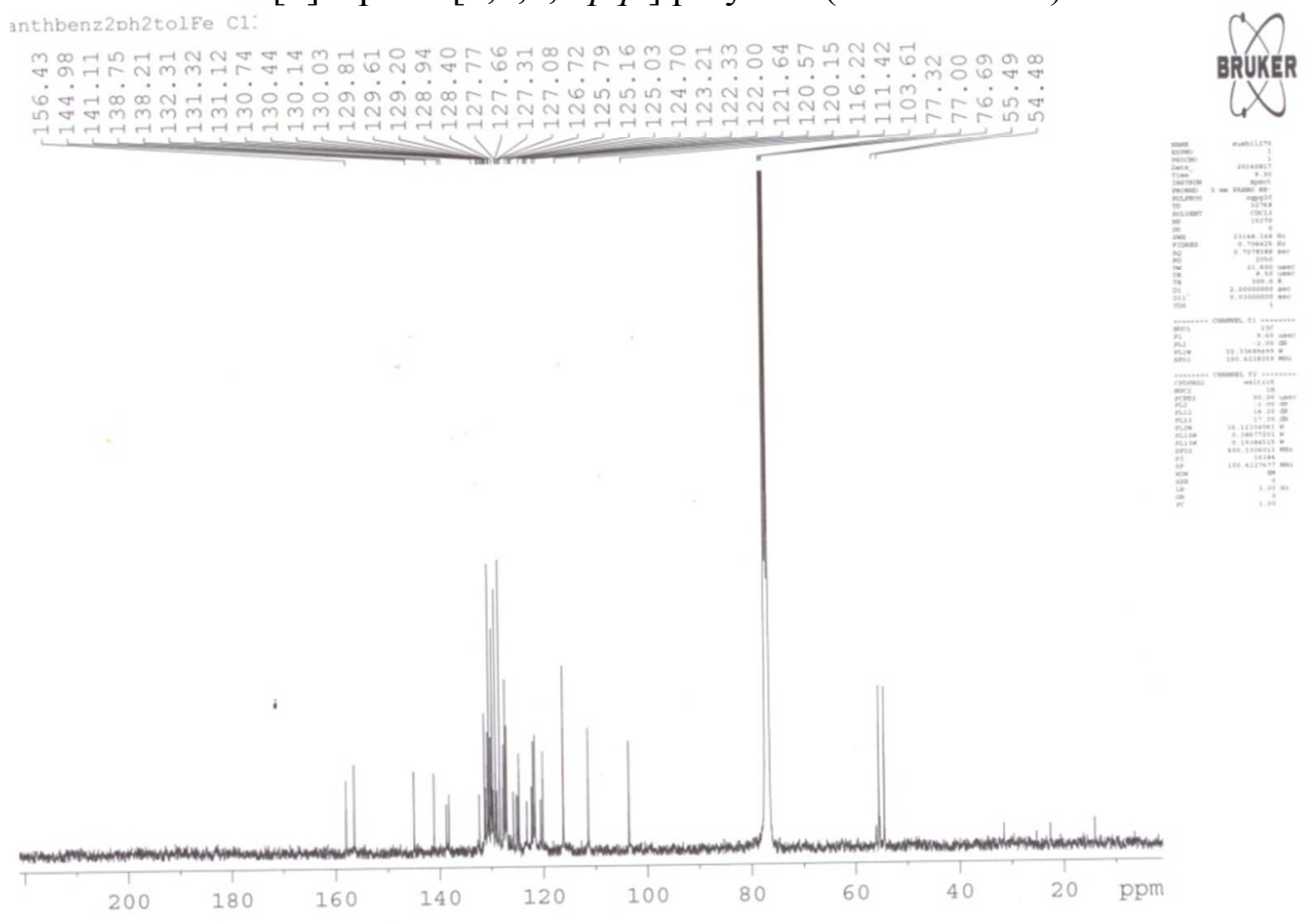

Figure S68. ${ }^{13} \mathrm{C}$ NMR spectrum of 2,7-dimethoxy-9,14diphenylbenzo[b]naphtho[1,2,3,4-pqr]perylene (Met-PhBNP) 


\section{Supporting Information}
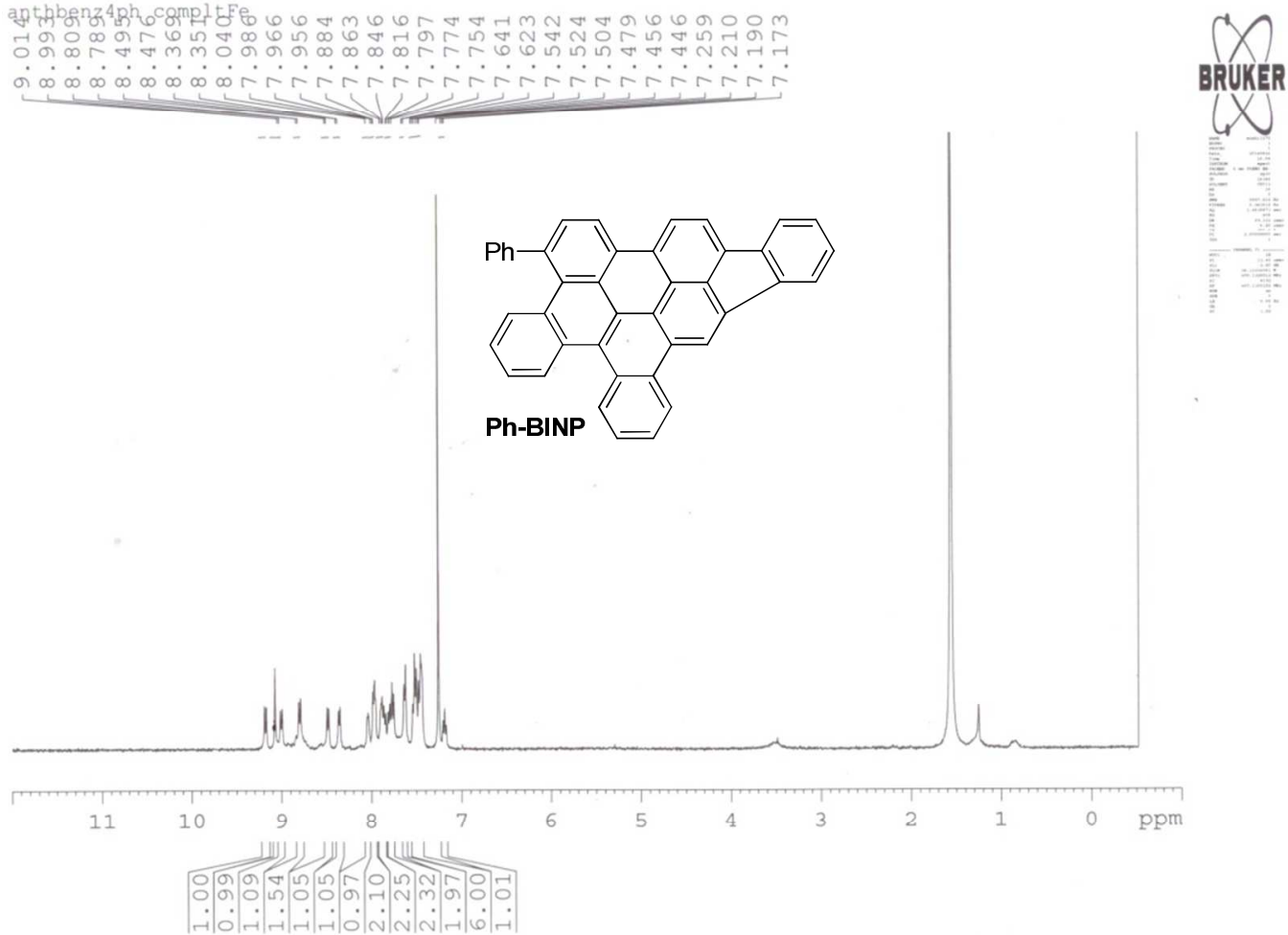

Figure S69. ${ }^{1} \mathrm{H}$ NMR spectrum of 18-phenylbenzo[ $\left.b\right]$ indeno[ $[1,2,3-l m]$ naphtho[1,2,3,4pqr]perylene (Ph-BINP)

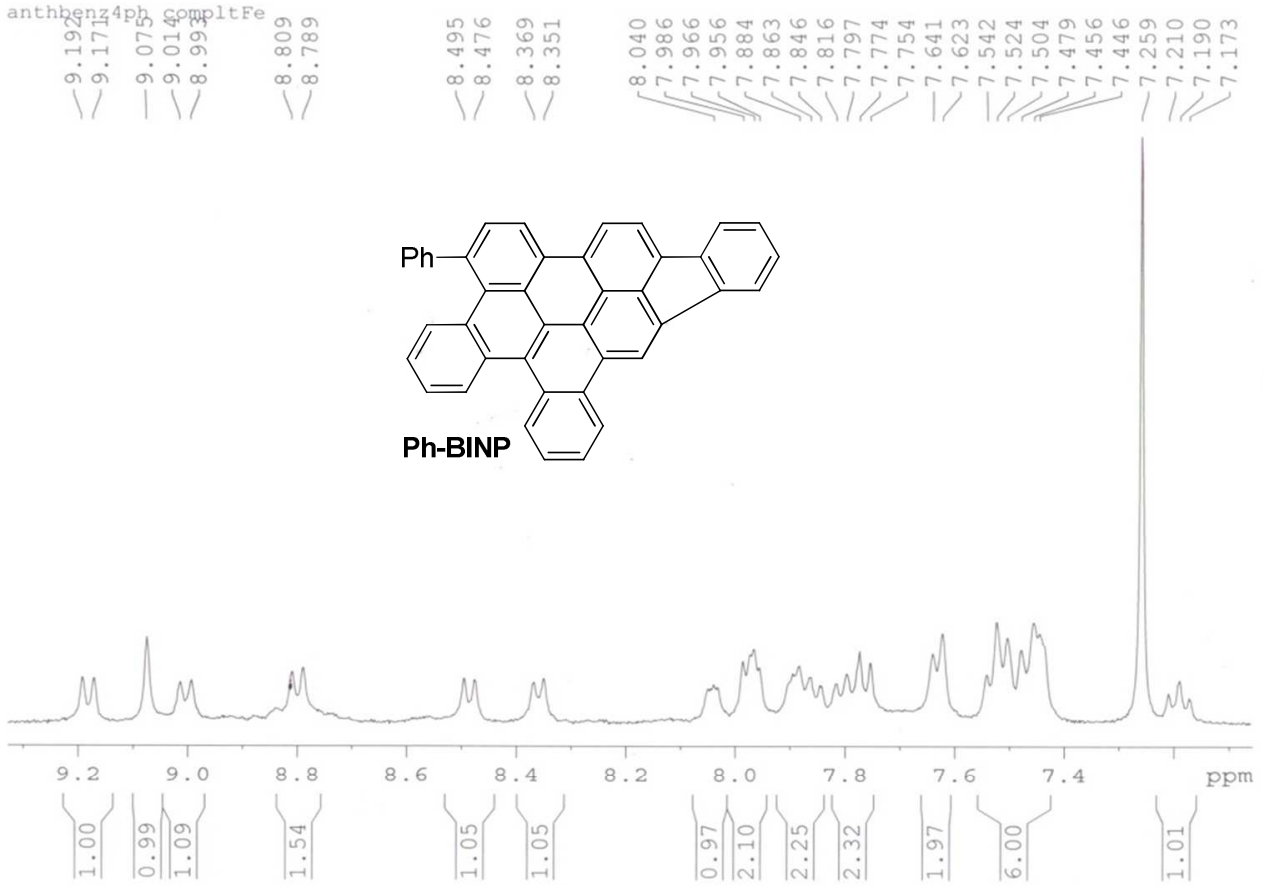

Figure S70. ${ }^{1} \mathrm{H}$ NMR spectrum (expansion) of 18-phenylbenzo[b]indeno[1,2,3lm]naphtho[1,2,3,4-pqr]perylene (Ph-BINP) 
Supporting Information

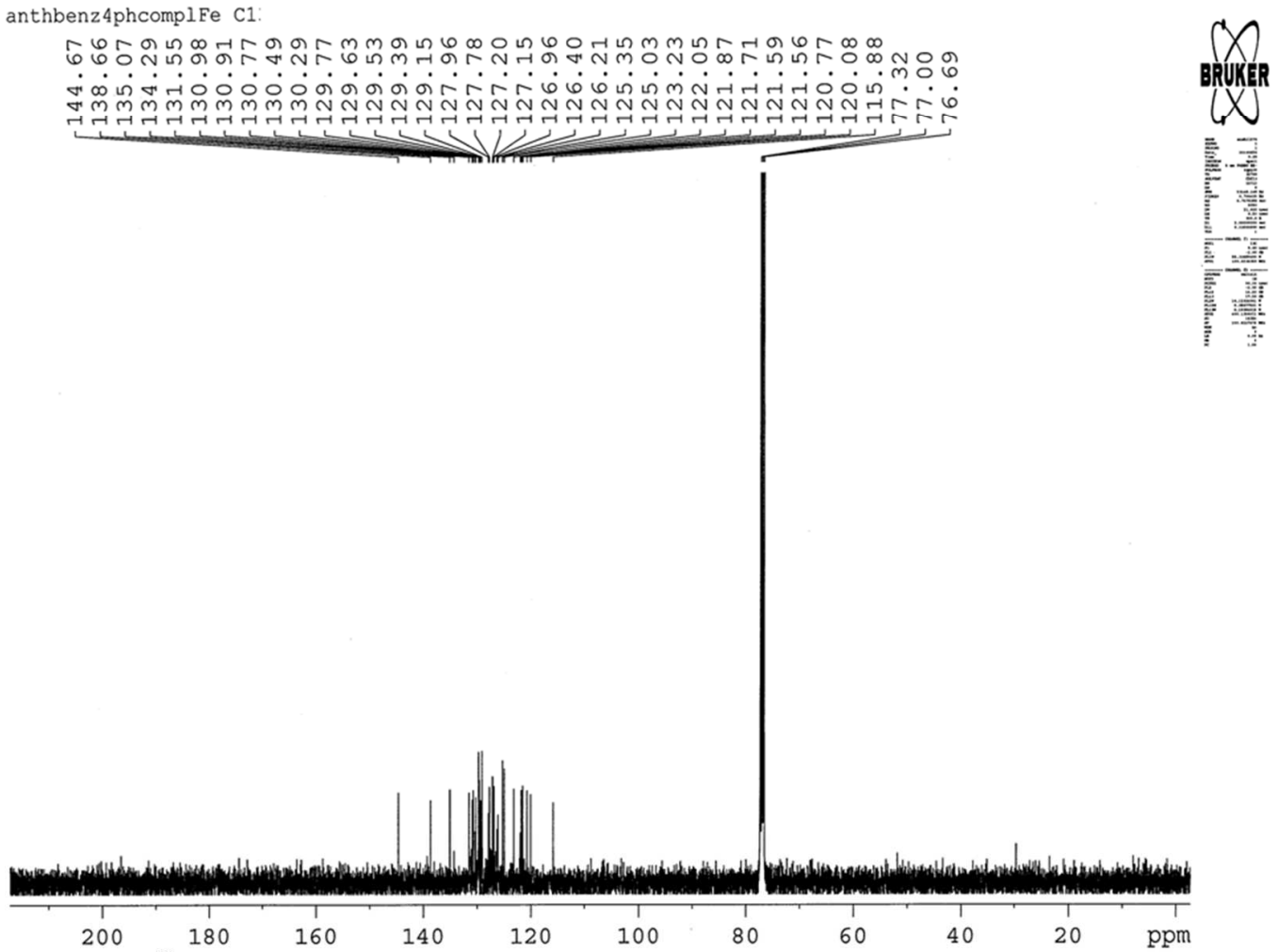

Figure S71. ${ }^{13} \mathrm{C}$ NMR spectrum of 18 -phenylbenzo[b]indeno[1,2,3-lm]naphtho[1,2,3,4pqr]perylene (Ph-BINP)

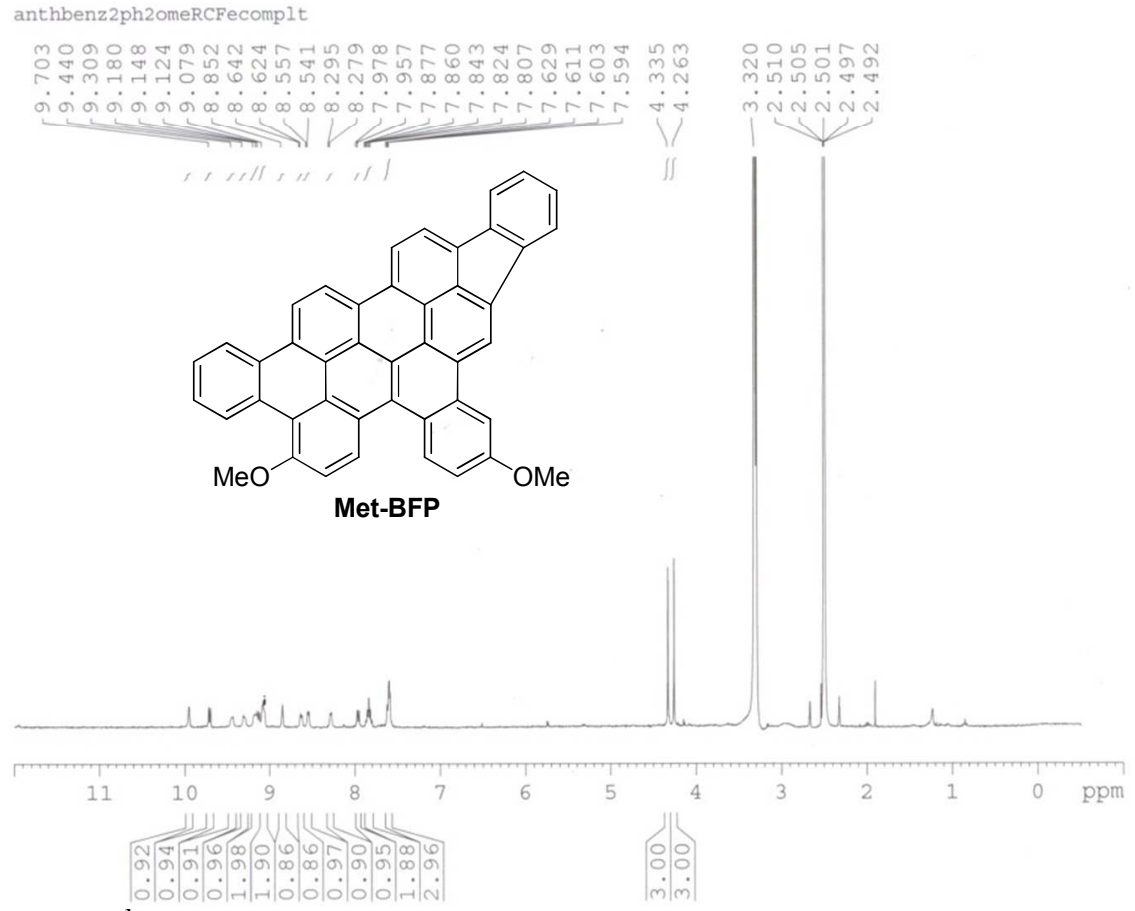

Figure S72. ${ }^{1} \mathrm{H}$ NMR spectrum of 5,10-dimethoxytribenzo[ $\left.a, g h i, k\right]$ fluorantheno[2,3,4$c d e]$ perylene (Met-BFP) 
Supporting Information
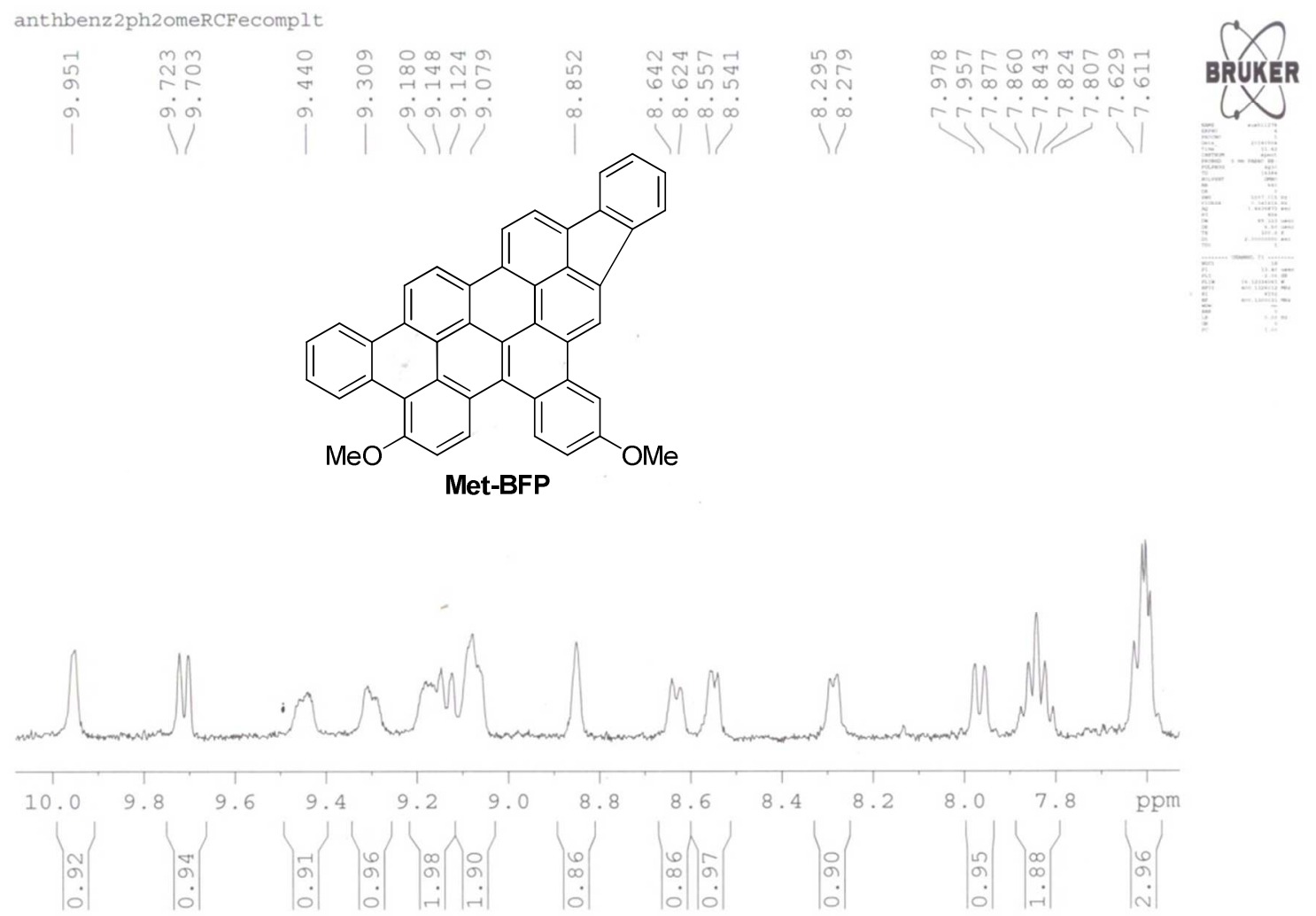

Figure S73. ${ }^{1} \mathrm{H}$ NMR spectrum (expansion) of 5,10dimethoxytribenzo[a,ghi,k]fluorantheno[2,3,4-cde]perylene (Met-BFP)

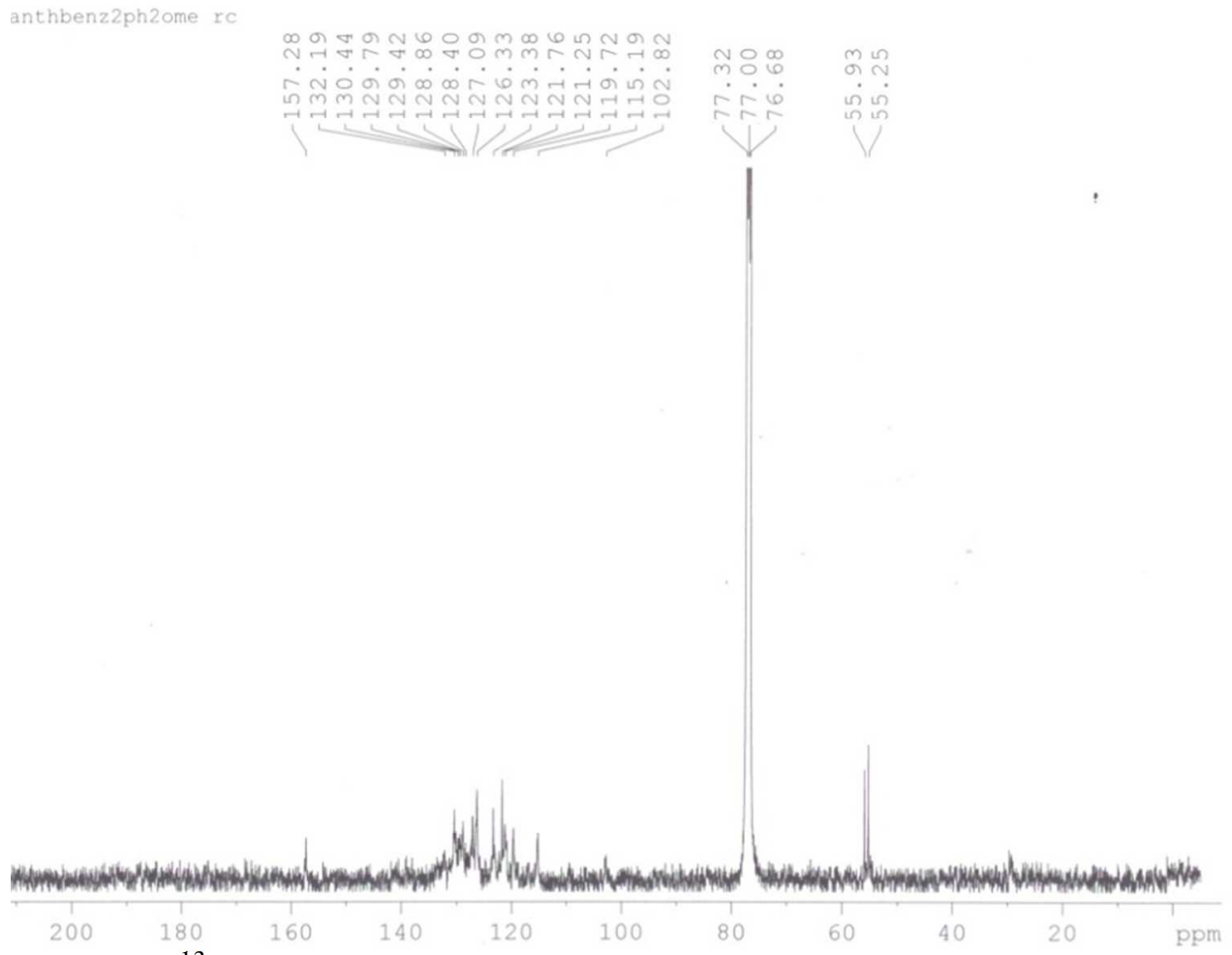

Figure S74. ${ }^{13} \mathrm{C}$ NMR spectrum of 5,10-dimethoxytribenzo[ $\left.a, g h i, k\right]$ fluorantheno[2,3,4cde]perylene (Met-BFP) 


\section{MALDI-TOF MS spectra of final derivatives}

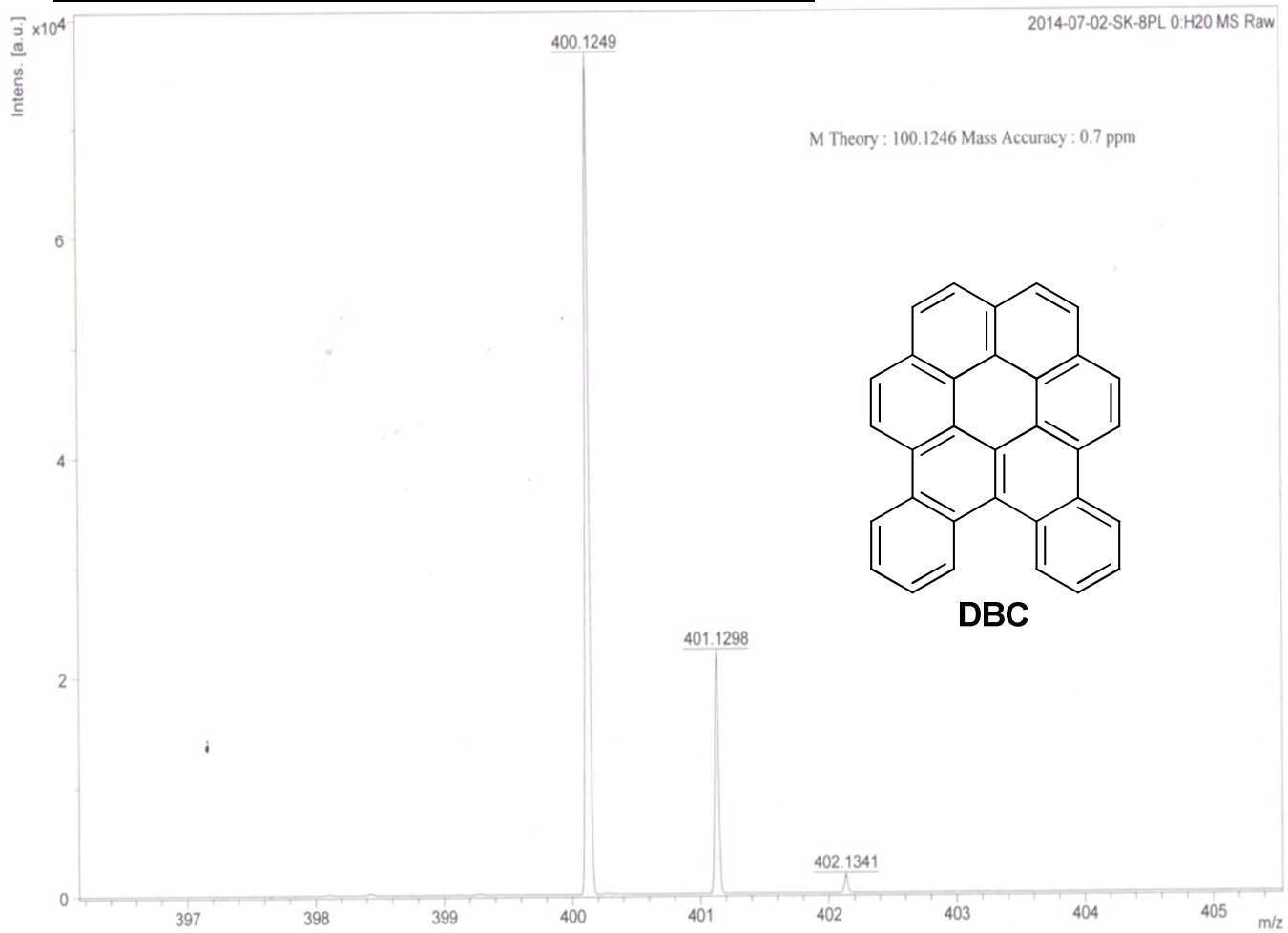

Figure S75. MALDI-TOF MS spectrum of Dibenzo[a,d]coronene (DBC)

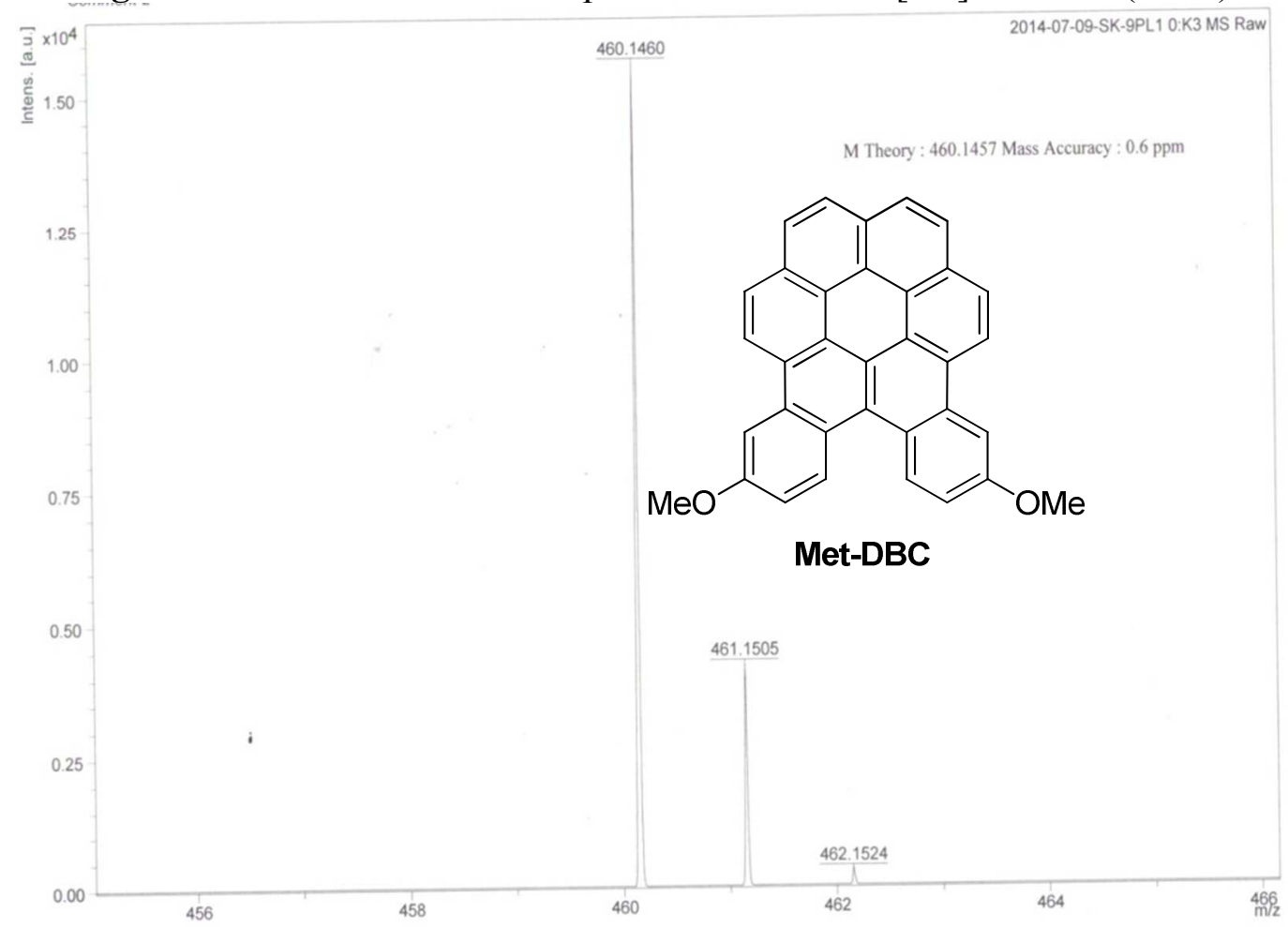

Figure S76. MALDI-TOF MS spectrum of 8,13-dimethoxydibenzo[a,d]coronene (MetDBC) 
Supporting Information

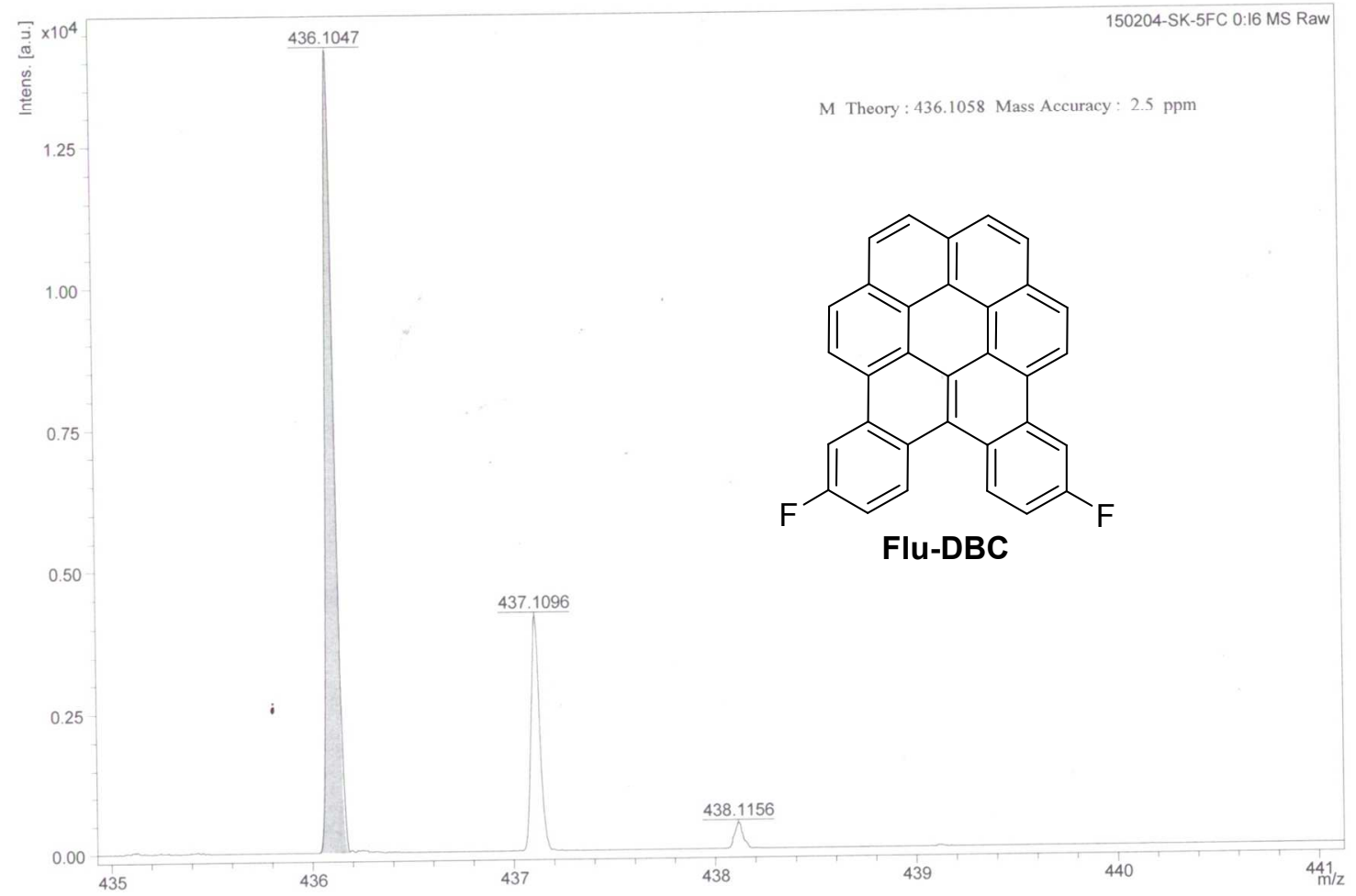

Figure S77. MALDI-TOF MS spectrum of 8,13-dimethoxydibenzo[a,d]coronene (FluDBC)

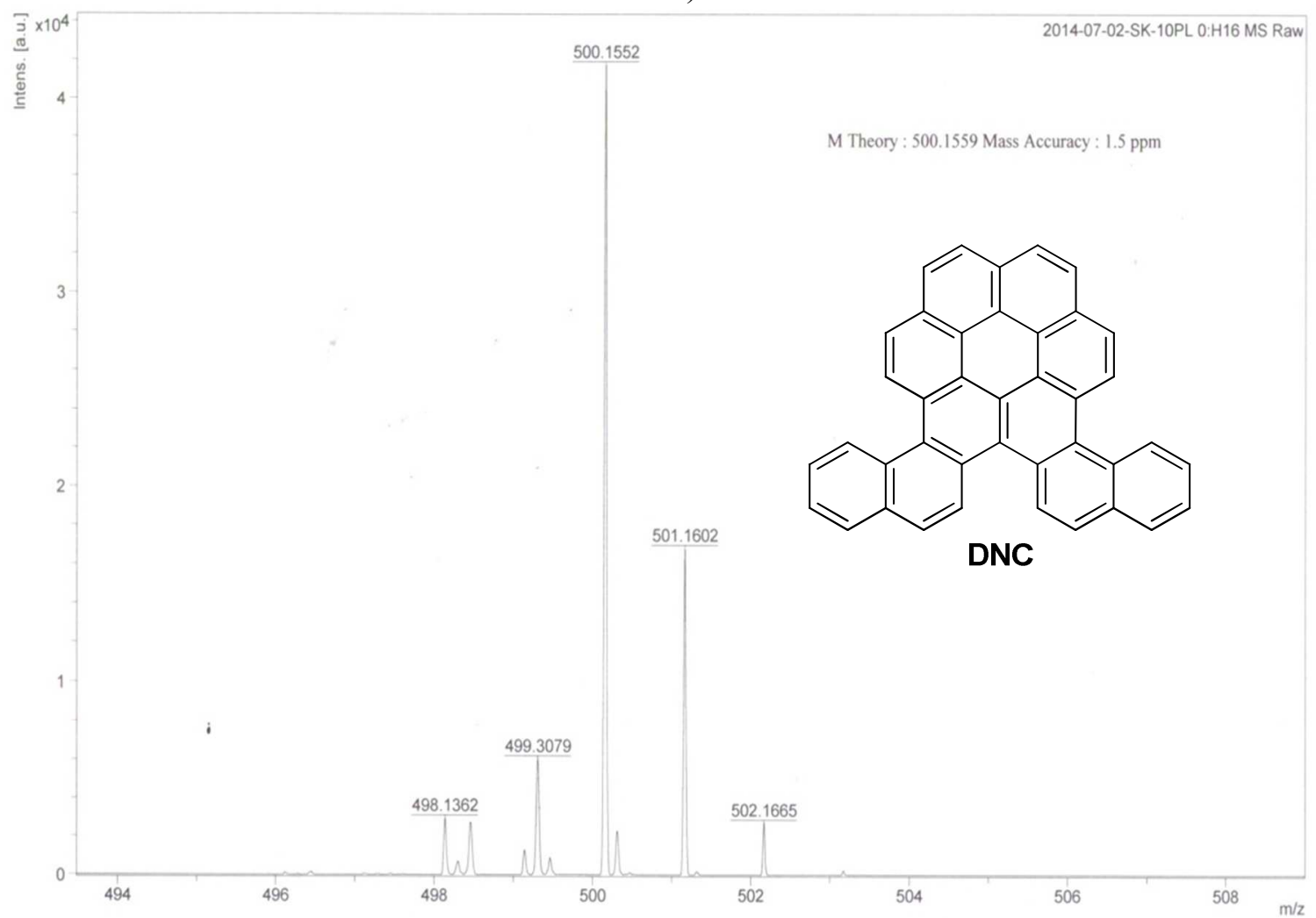

Figure S78. MALDI-TOF MS spectrum of dinaphtho[1,2-a:2',1'-d]coronene (DNC) 
Supporting Information

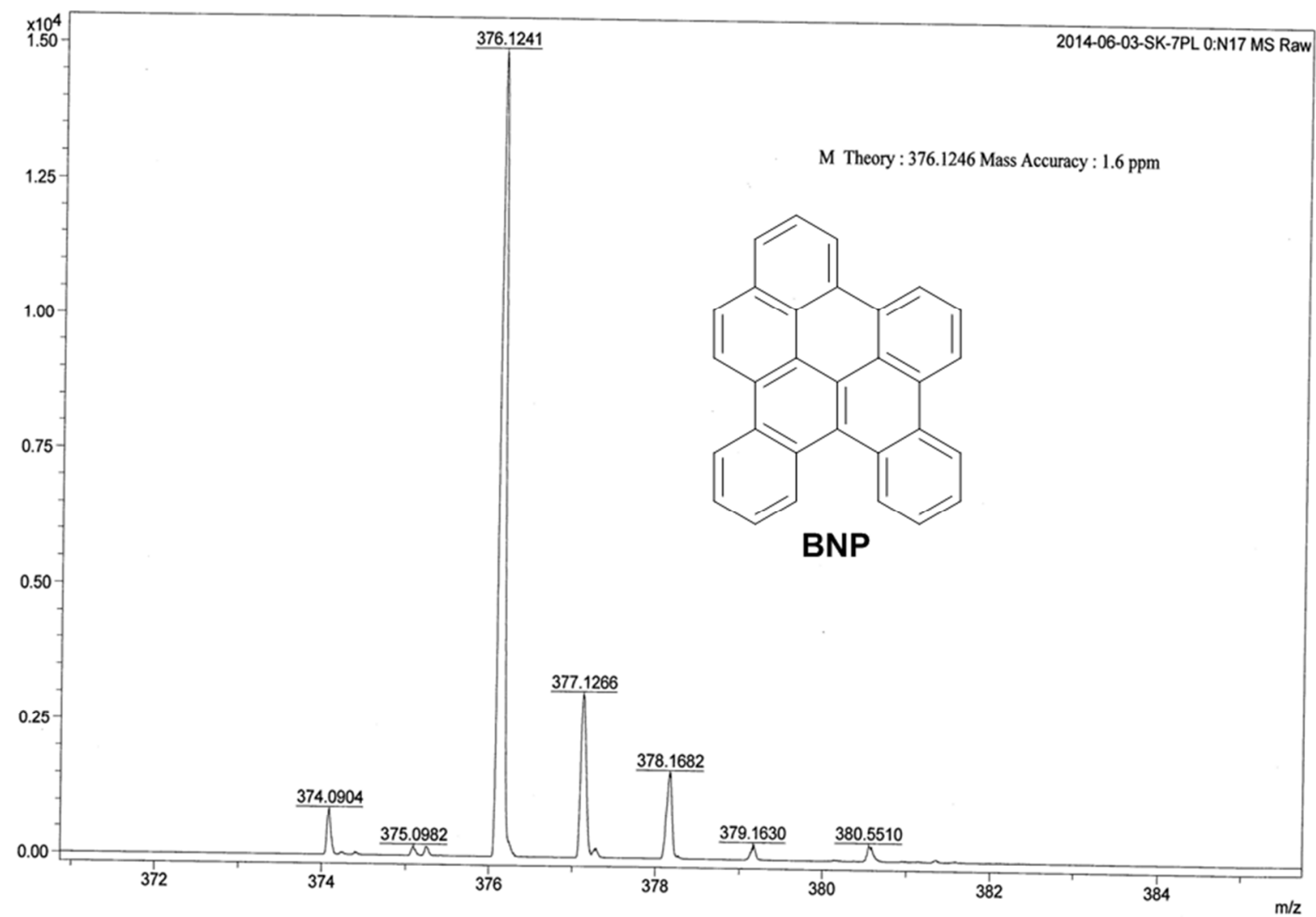

Figure S79. MALDI-TOF MS spectrum of benzo[b]naphtho[1,2,3,4-pqr]perylene (BNP)

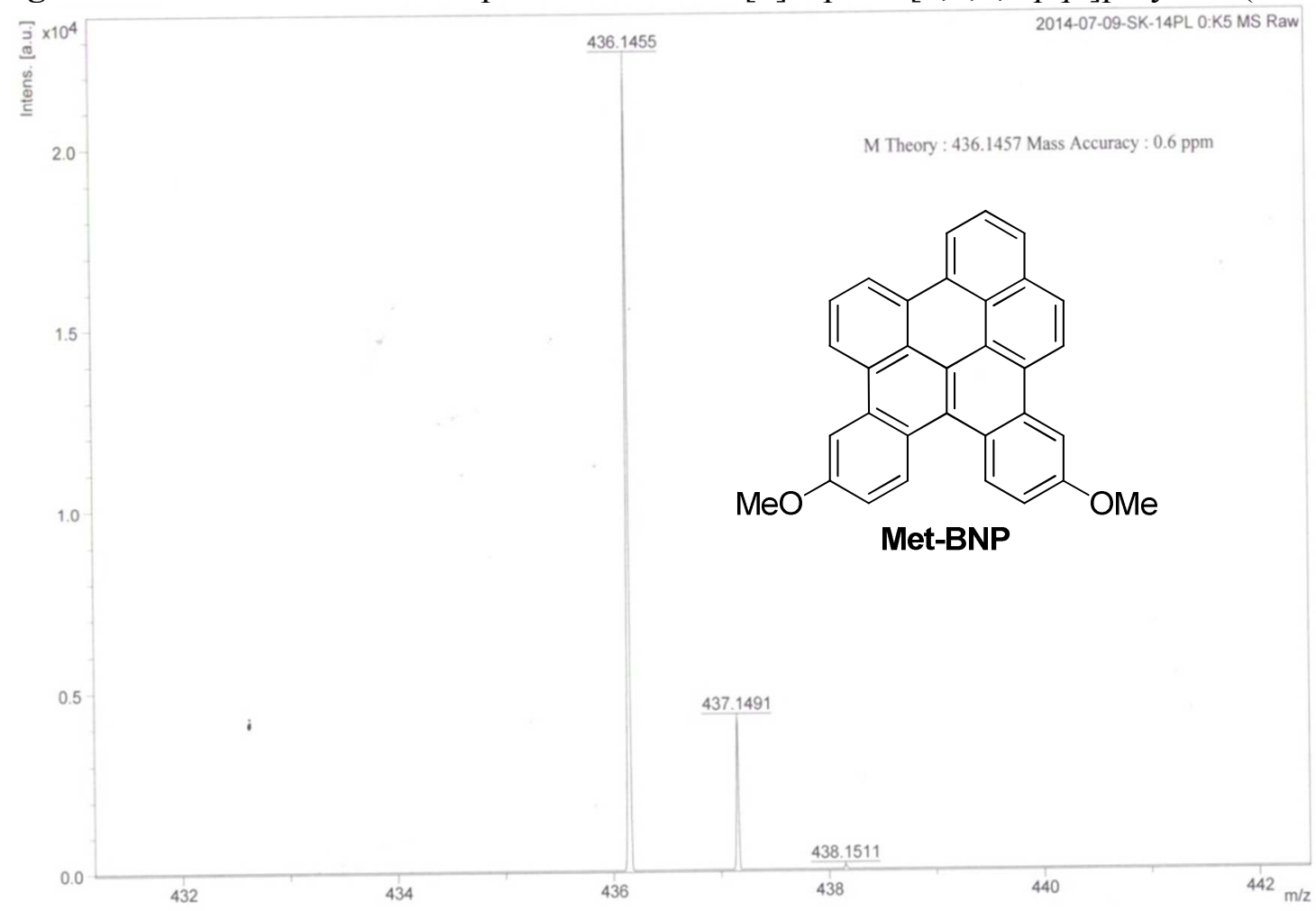

Figure S80. MALDI-TOF MS spectra of 2,7-dimethoxybenzo[b]naphtho[1,2,3,4pqr]perylene (Met-BNP) 
Supporting Information

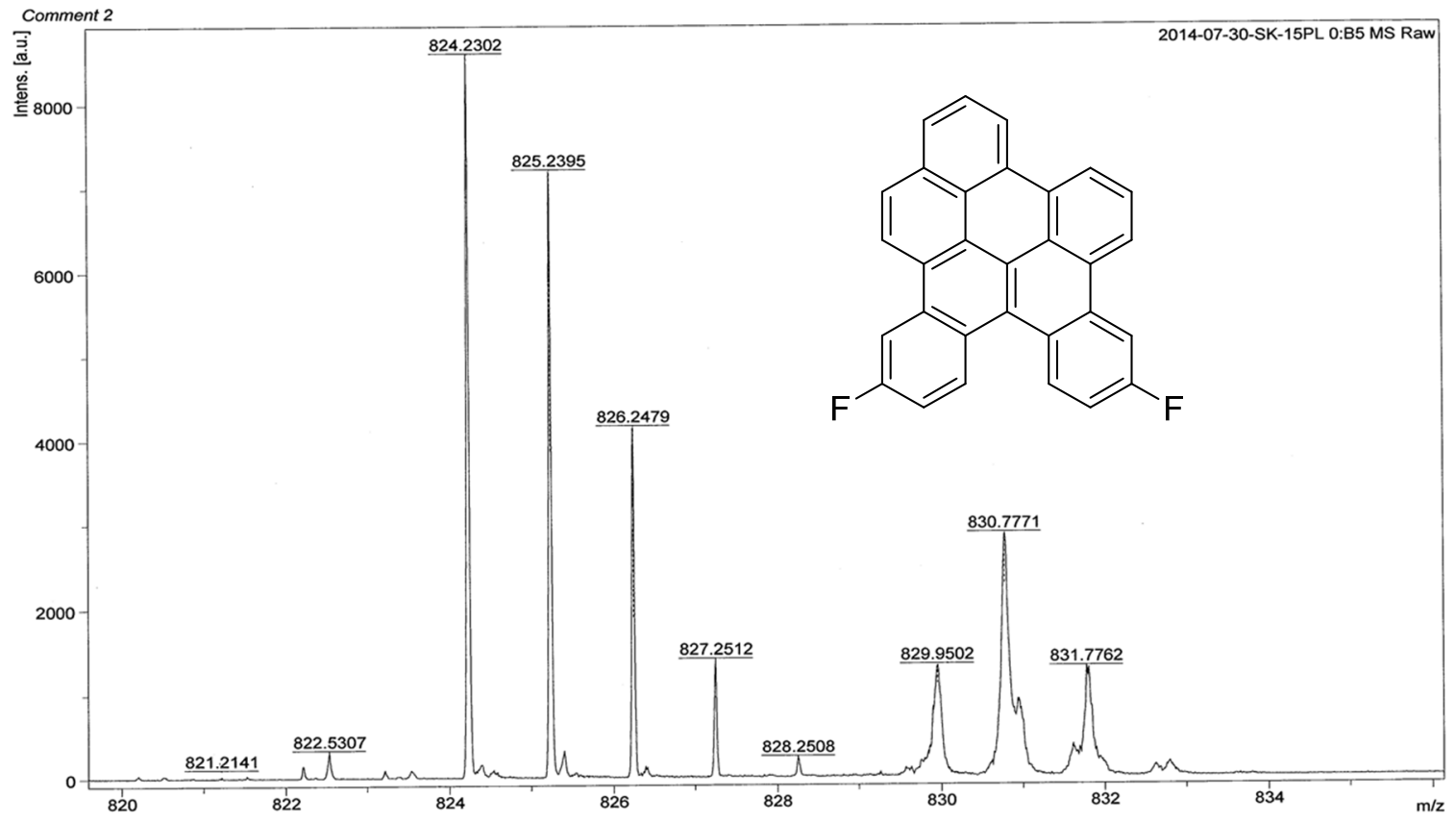

Figure S81. Maldi-Tof mass spectrum of 2,7-difluorobenzo[b]naphtho[1,2,3,4pqr]perylene (Flu-BNP)

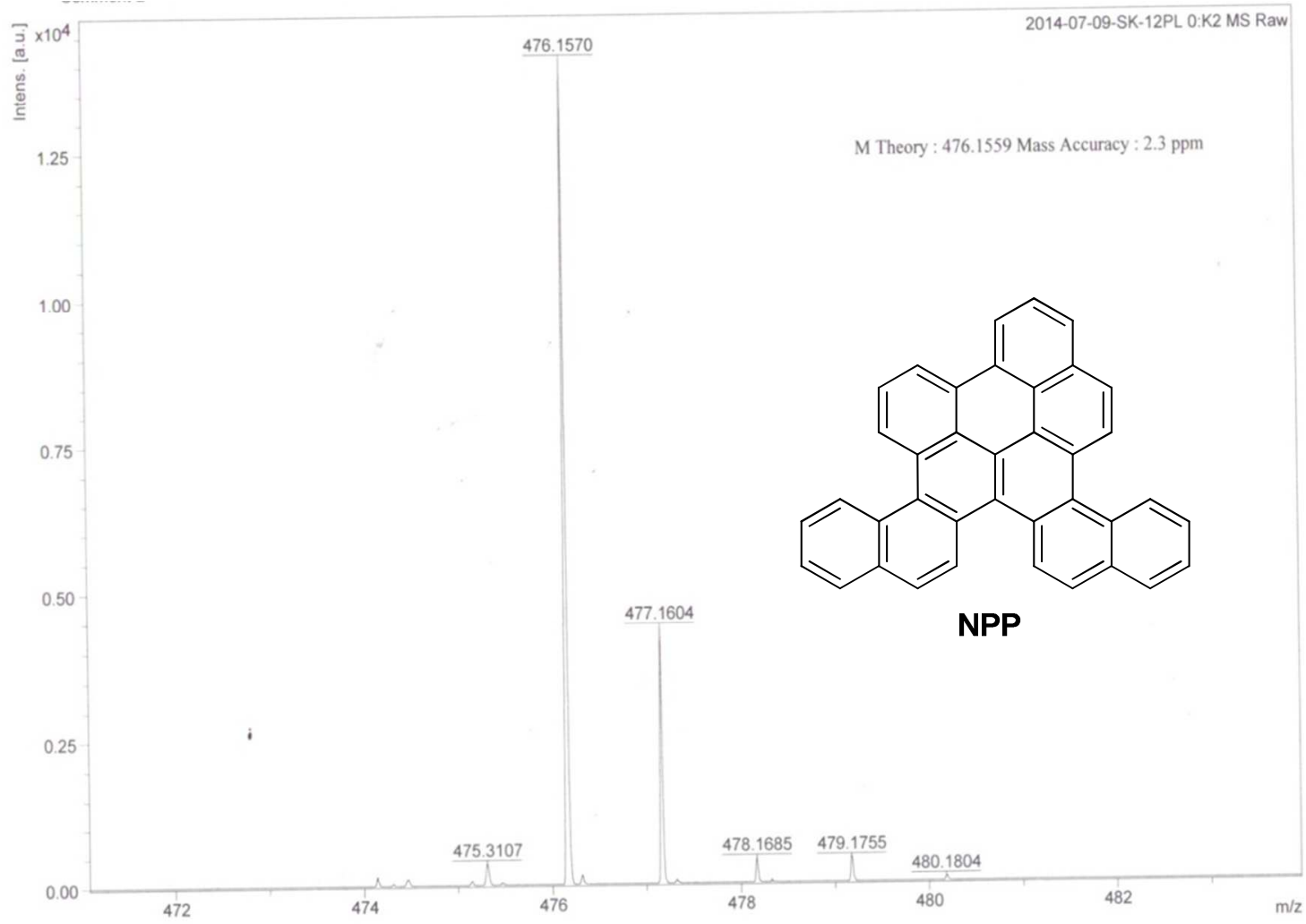

Figure S82. MALDI-TOF MS spectrum of naphtho[2,1- $b]$ phenanthro[4,3,2,1pqr]perylene (NPP) 
Supporting Information

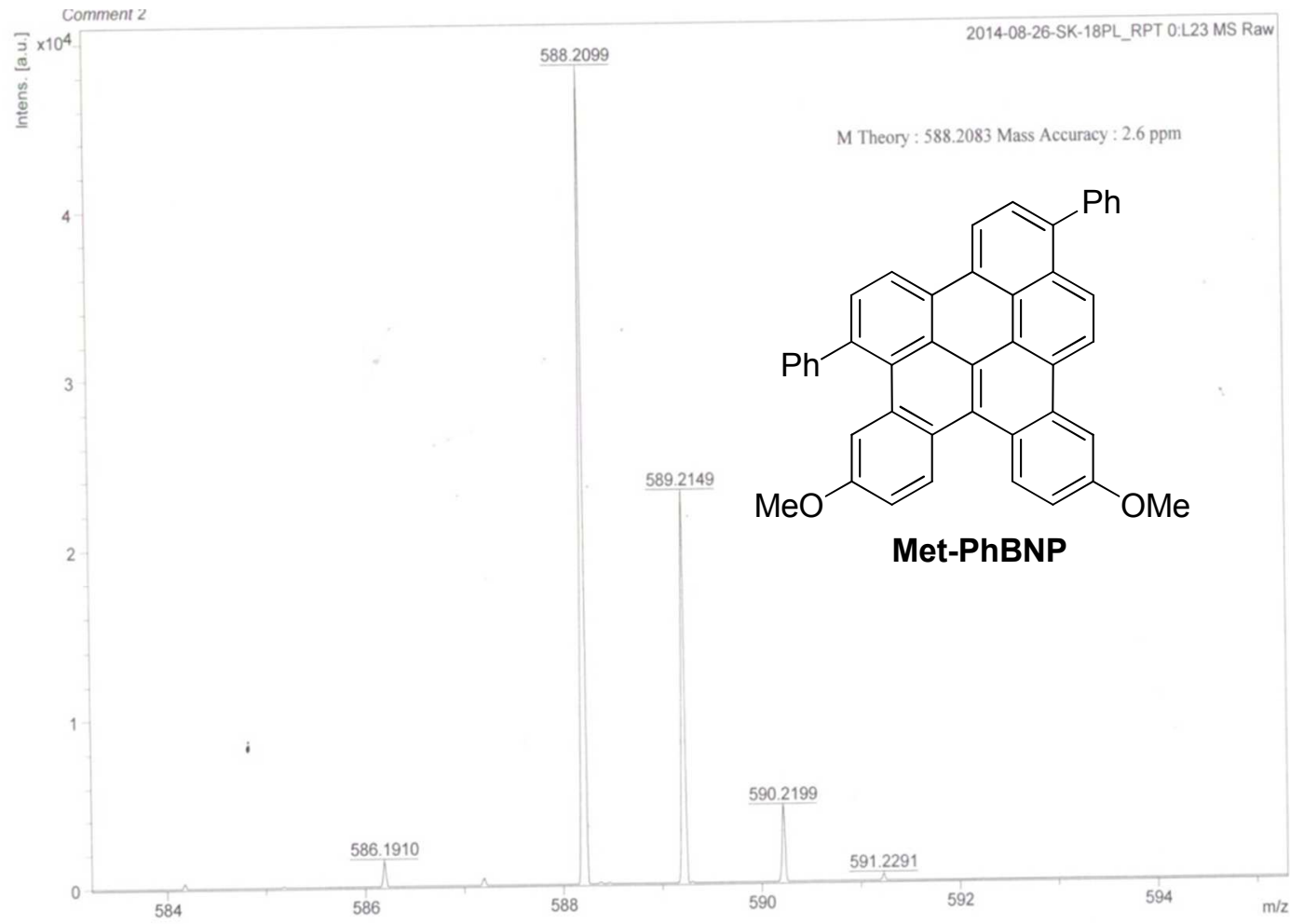

Figure S83. MALDI-TOF MS spectrum of 2,7-dimethoxy-9,14diphenylbenzo[b]naphtho[1,2,3,4-pqr]perylene (Met-PhBNP)

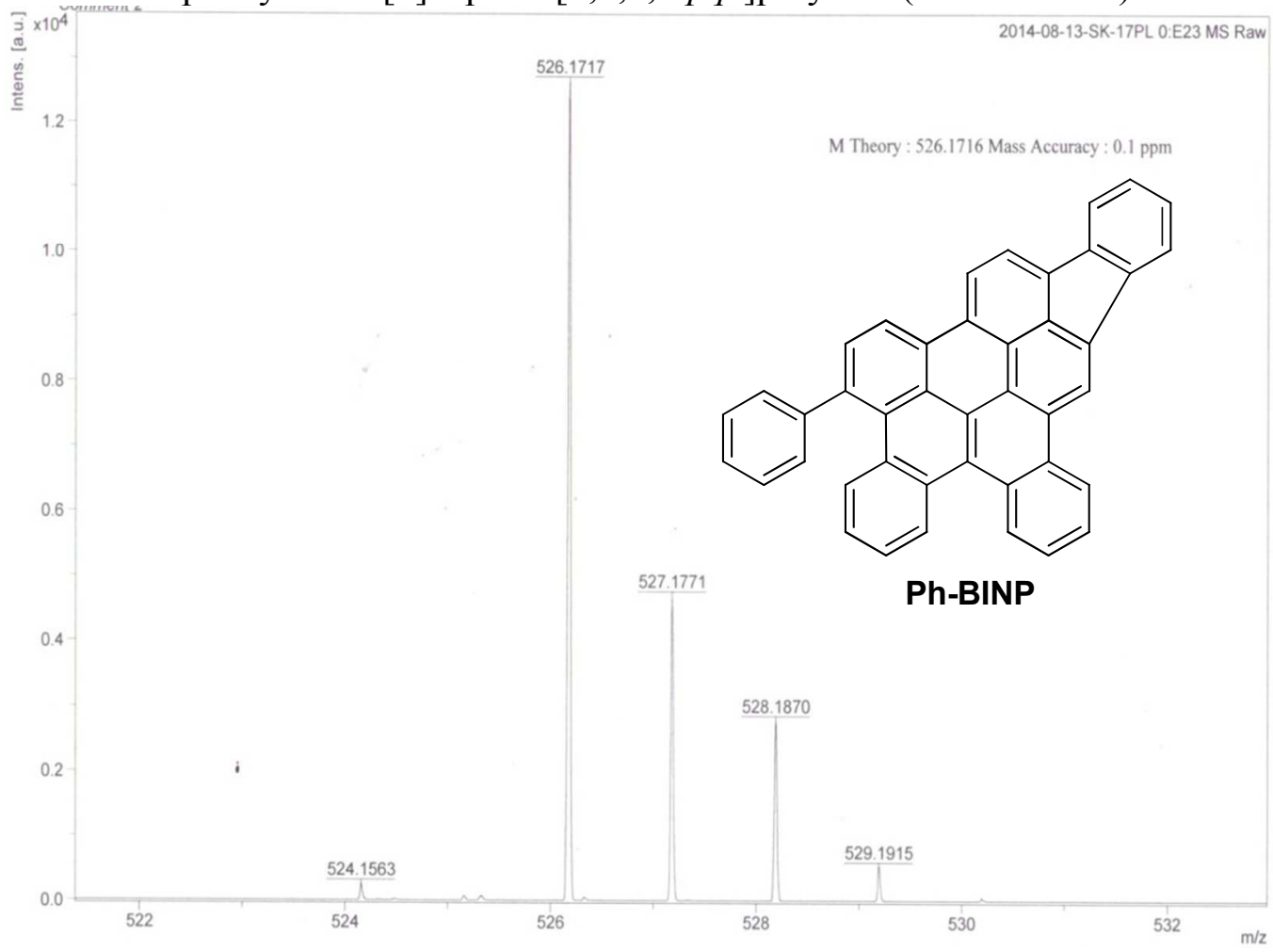

Figure S84. MALDI-TOF MS spectrum of 18-phenylbenzo[b]indeno[1,2,3lm]naphtho[1,2,3,4-pqr]perylene (Ph-BINP) 
Supporting Information

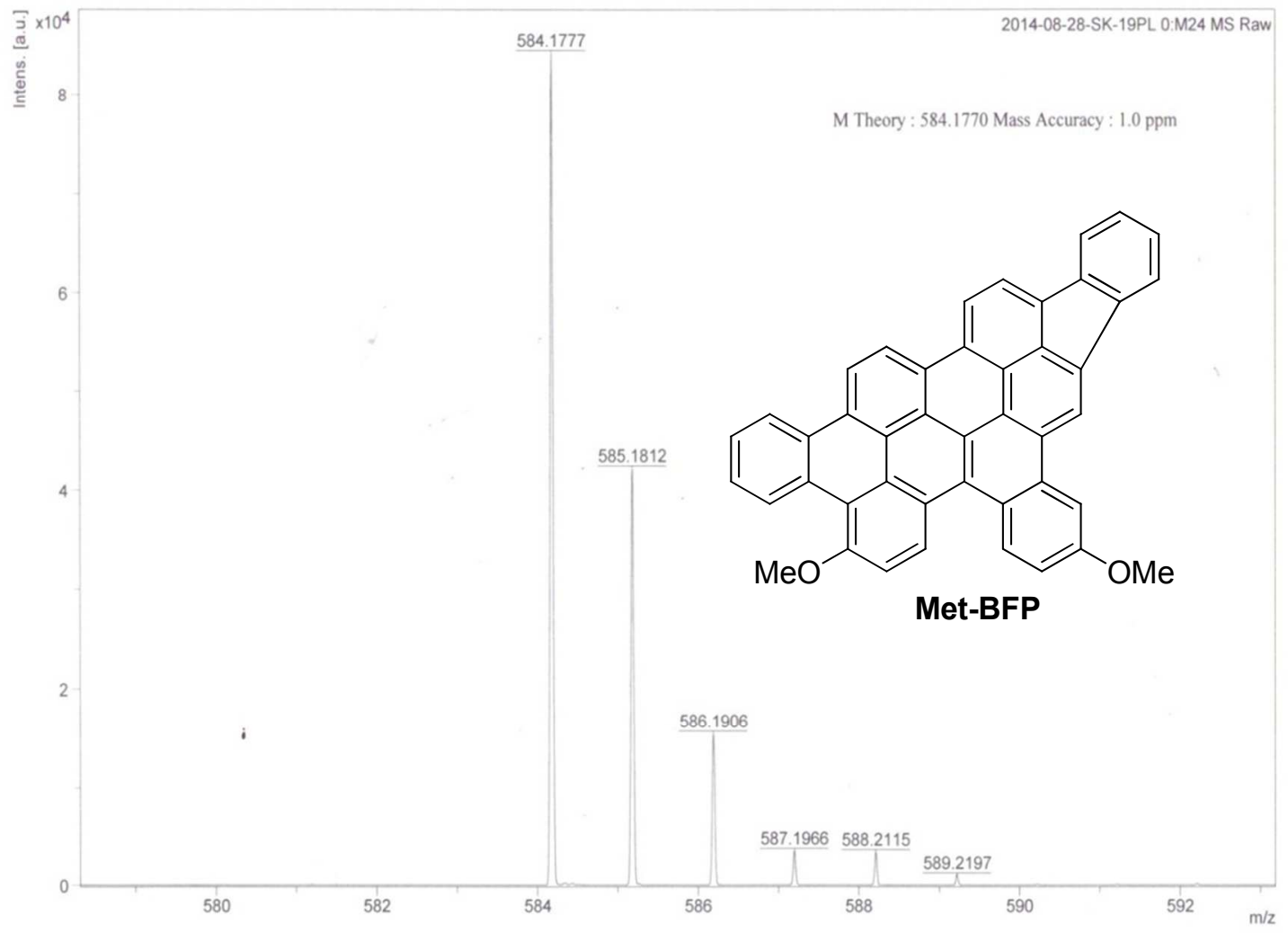

Figure S85. MALDI-TOF MS spectrum of 5,10dimethoxytribenzo[ $[a, g h i, k]$ fluorantheno[2,3,4-cde]perylene (Met-BFP)

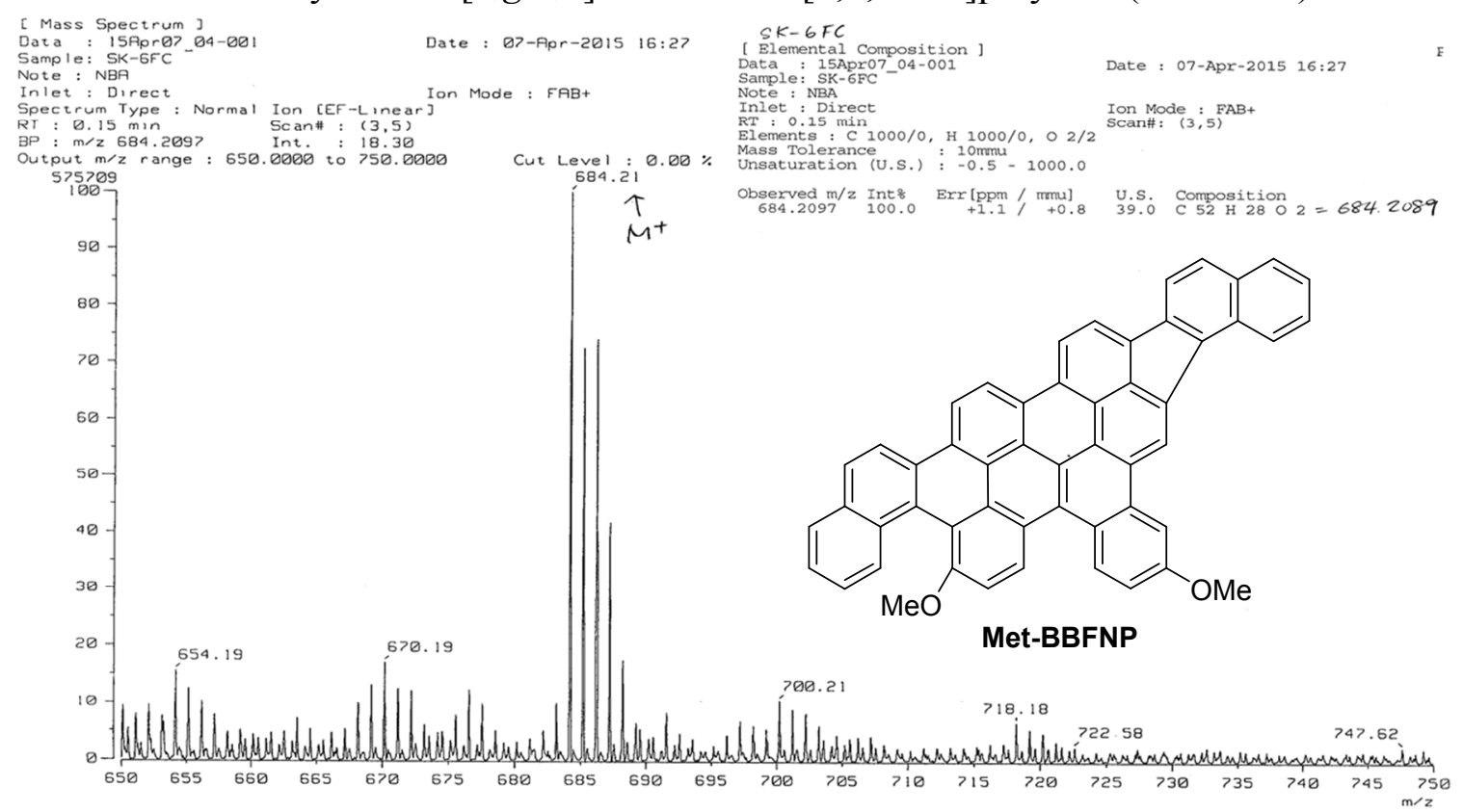

Figure S86. High resolution Fab-spectrum 5,10-dimethoxy dibenzo[a,ghi] benzo[9,10] fluorantheno[2,3,4-cde]naphtho[2,1-k]perylene (Met-BBFNP) 
6. Single-crystal data and packing in DBC, Flu-DBC, DNC and Ph-BINP molecules.

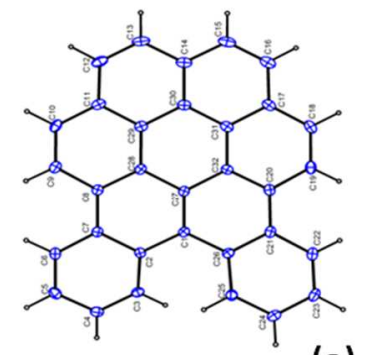

(a)

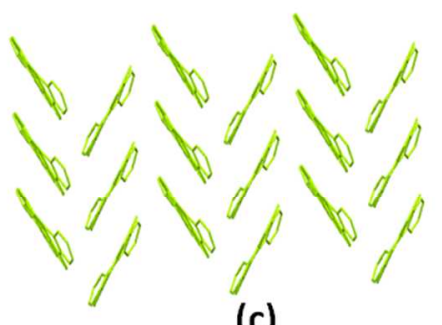

(c)

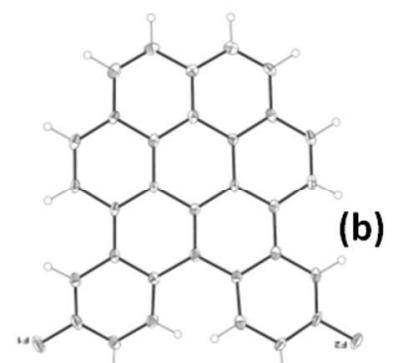
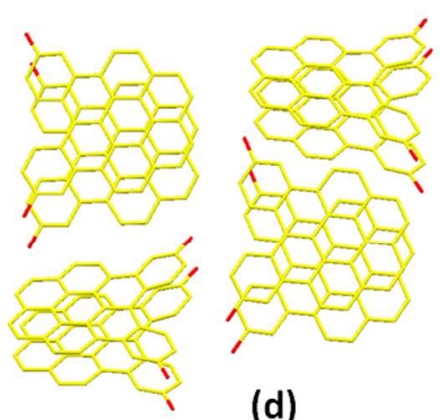

(d)

Figure S87 (a-b). Thermal ellipsoids of DBC (52\%) and Flu-DBC (48\%); (c-d) Packing of molecules in DBC and Flu-DBC

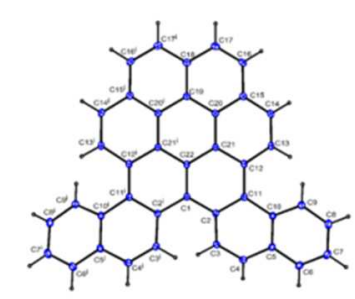

(a)

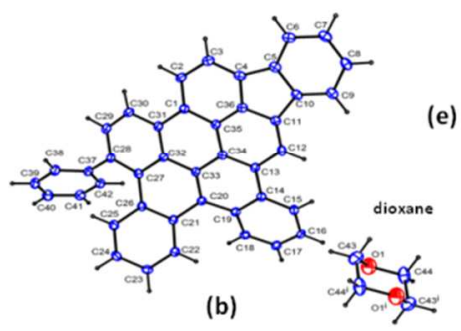

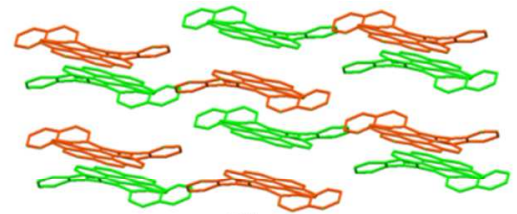

(c)

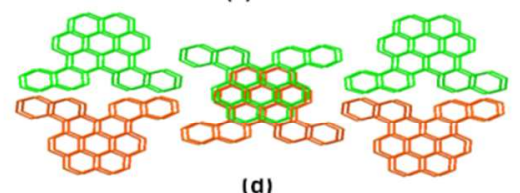

(d)

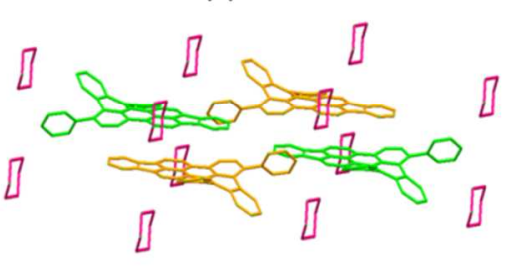

Figure S88. (a-b) ORTEP plots of DNC (51\%) and Ph-BINP (46\%); (c-d) Packing arrangement in DNC; (e) Packing arrangement in Ph-BINP. 
Supporting Information

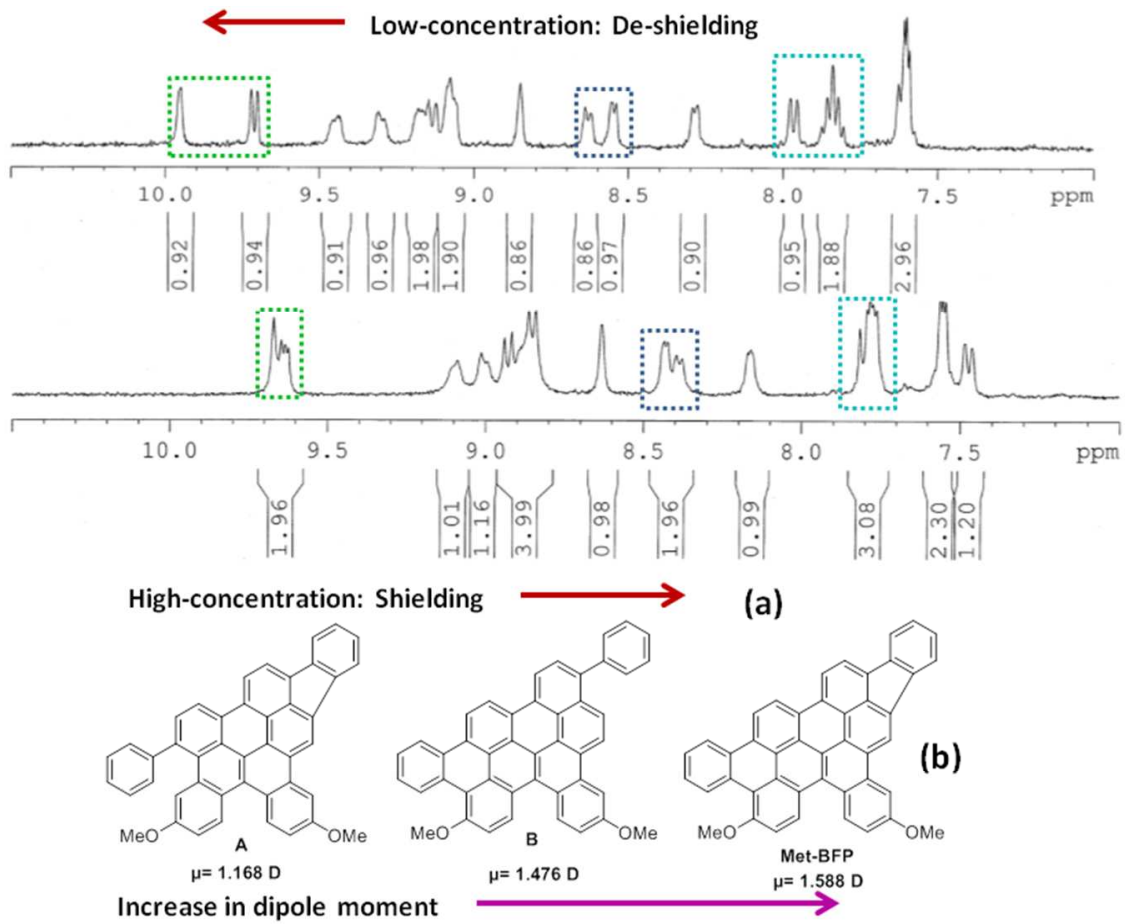

Figure S89. NMR spectra of Met-BFP recorded at different concentrations in DMSO- $\mathrm{d}_{6}$; (b) estimated dipole moment change upon fusion into planar Met-BFP from its precursors.

\section{Absorption/emission spectra of final derivatives as recorded in} dichloromethane

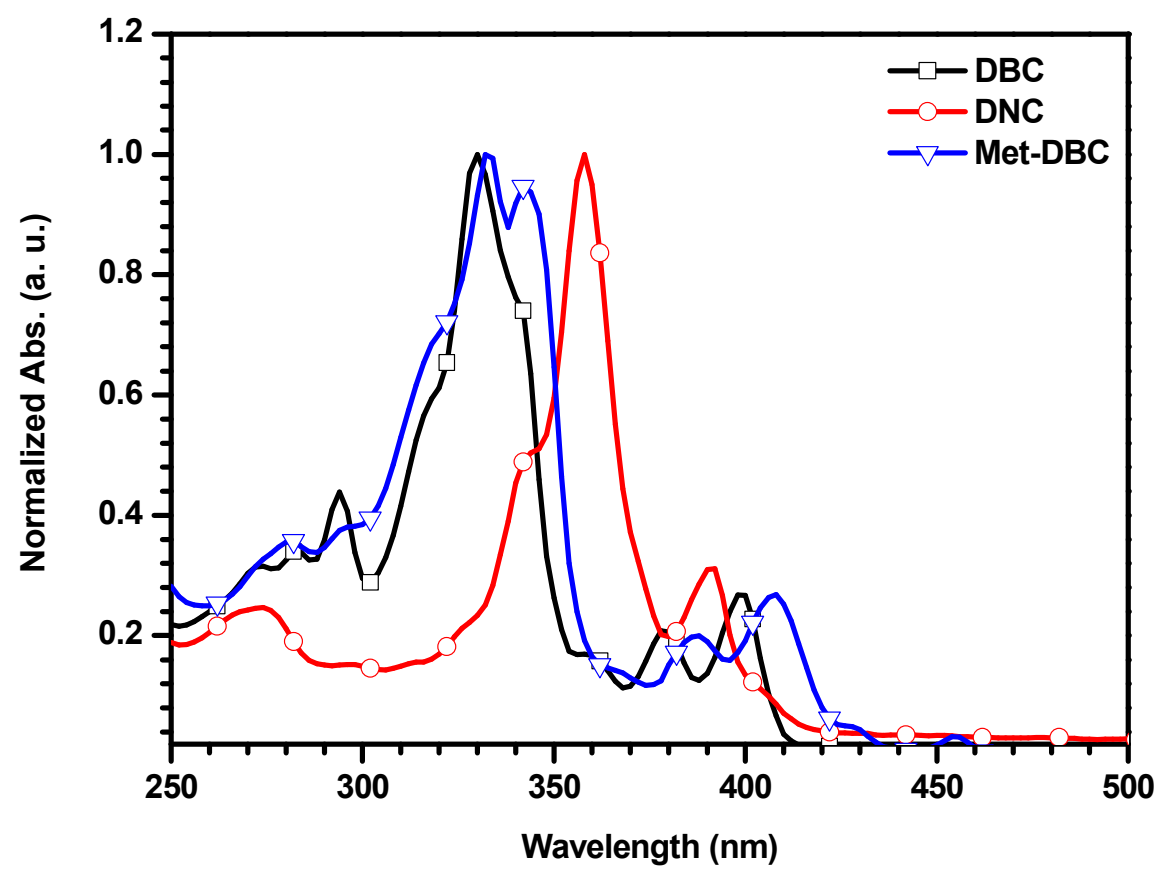

Figure S90. Normalized absorption spectra of coronene derivatives 
Supporting Information

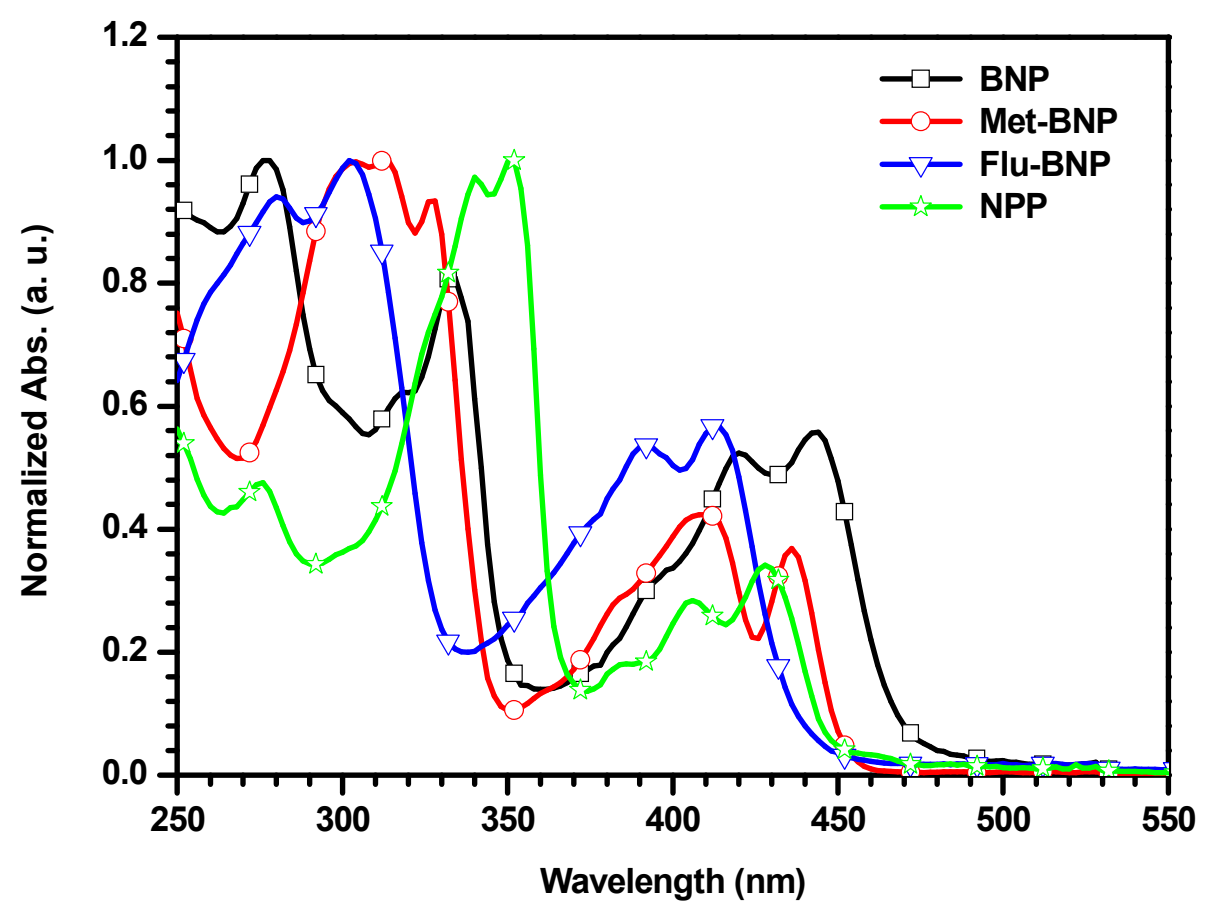

Figure S91. Normalized absorption spectra of perylene derivatives

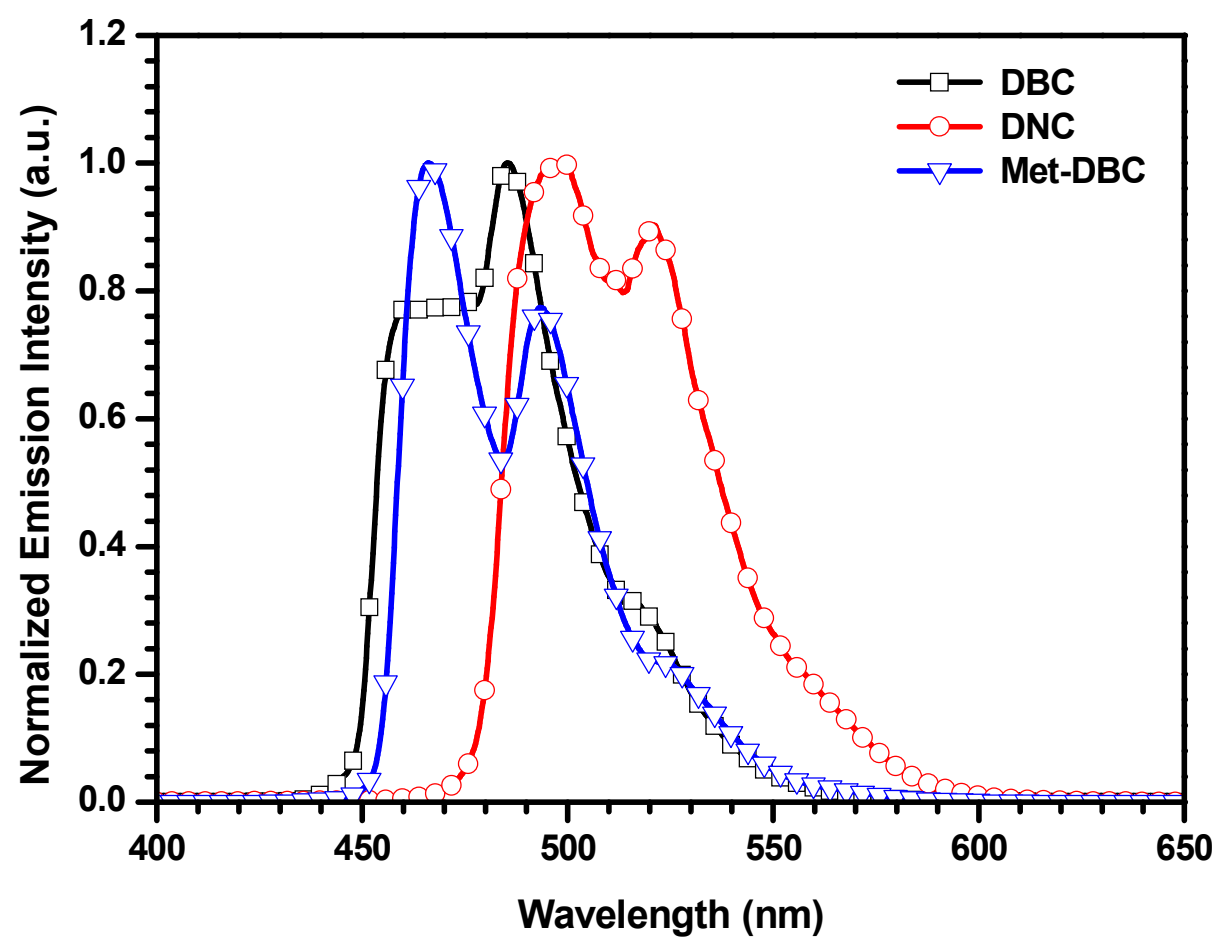

Figure S92. Normalized emission spectra of coronene derivatives 
Supporting Information

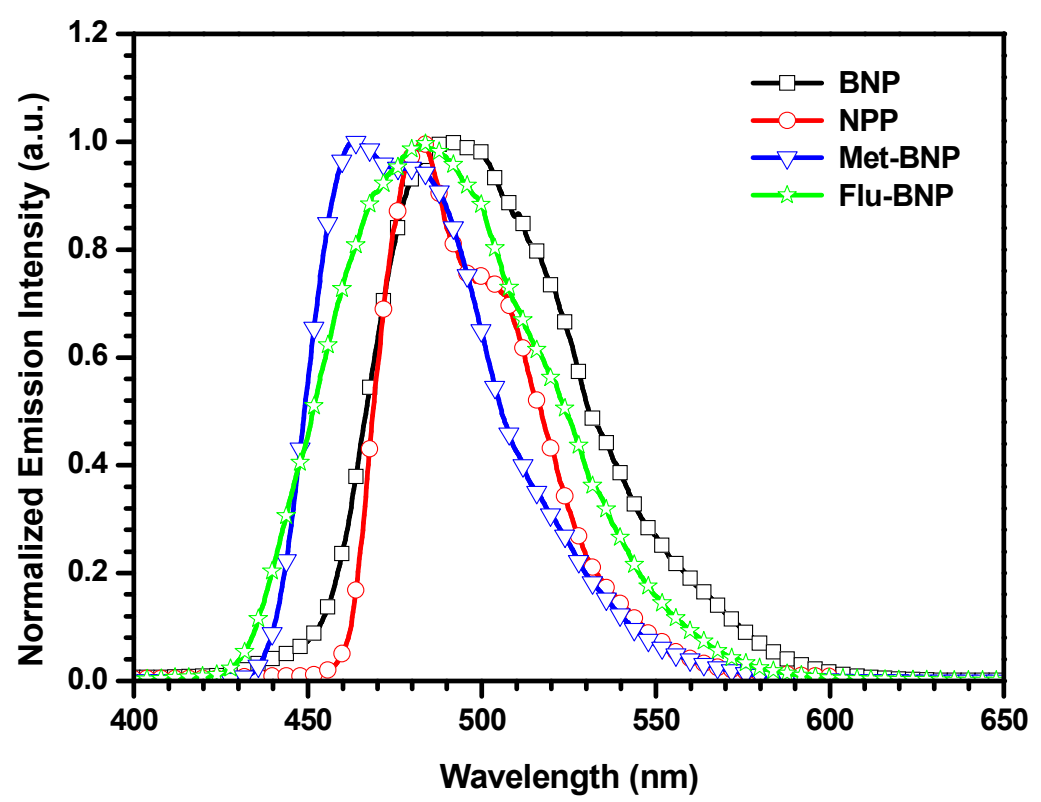

Figure S93. Normalized emission spectra of perylene derivatives

\section{Thermogravimetric plots of DBC and Flu-DBC}

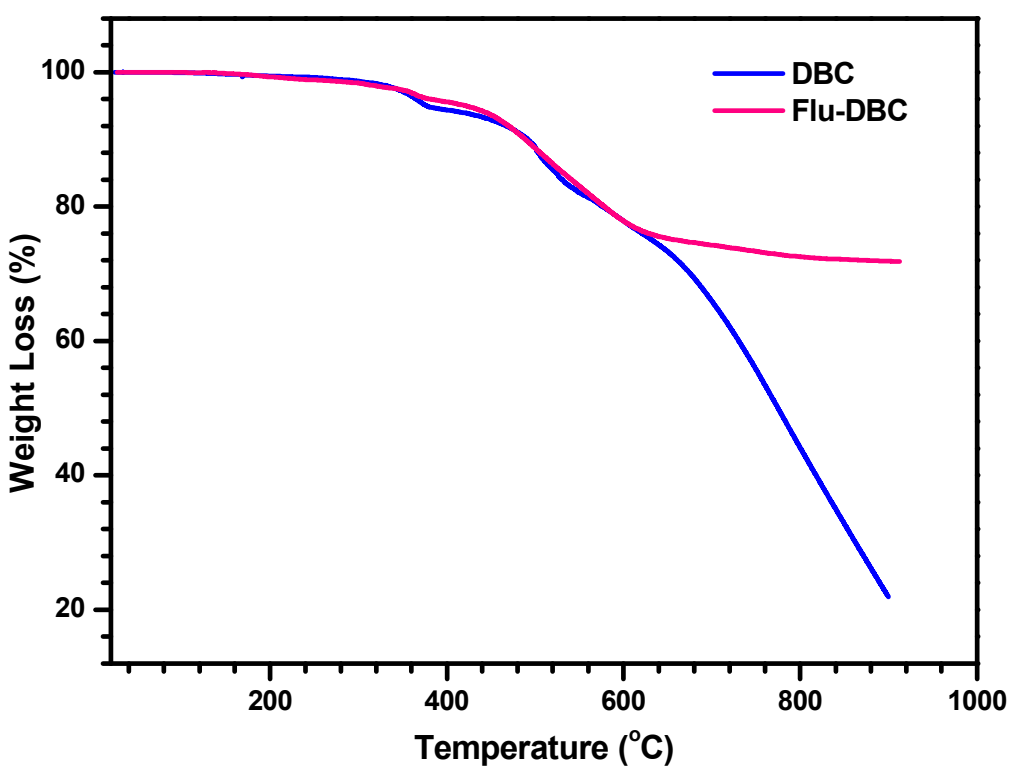

Figure S94. Thermogravimetric plot of DBC and Flu-DBC 
9. HOMO/LUMO plots of coronene- and perylene-embedded derivatives

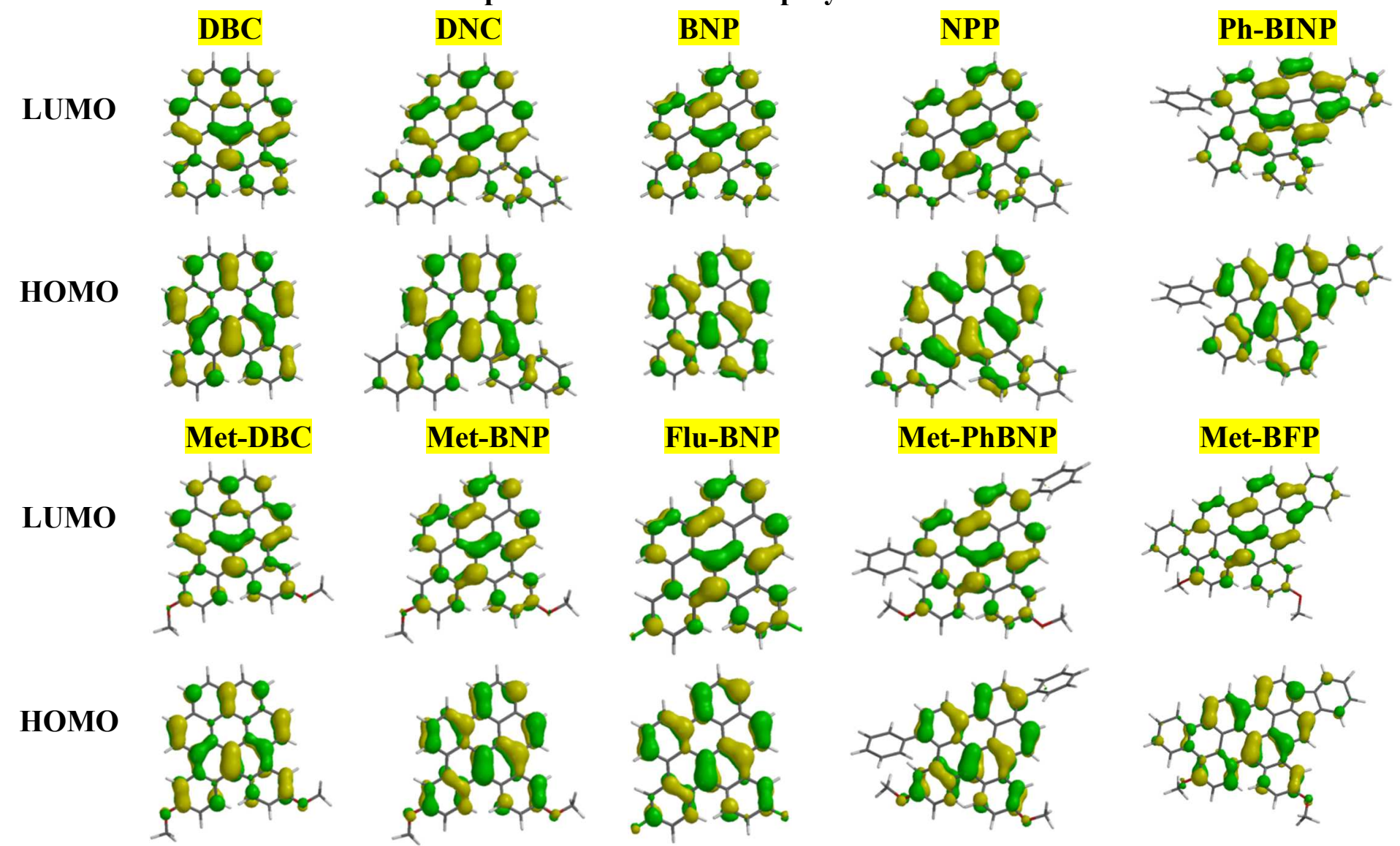

Figure S95. HOMO/LUMO plots of coronene and perylene derivatives (Obtained by Spartan pro software by semi-empirical method at level AM1) 
10. Single-crystal data, packing and device performance data for the DBC and Flu$\underline{\text { DBC }}$

\section{$\operatorname{DBC}(\mu)$}

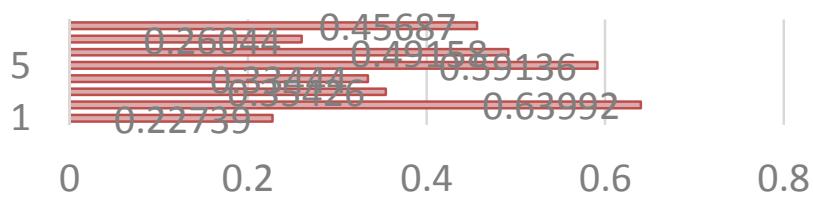

$\square$ Mobility

Figure S96. The mobility data obtained for different devices made by using DBC

\section{$\mathrm{DBC}(\mathrm{Vth})$}

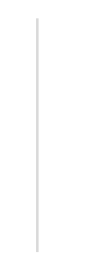

$-40$

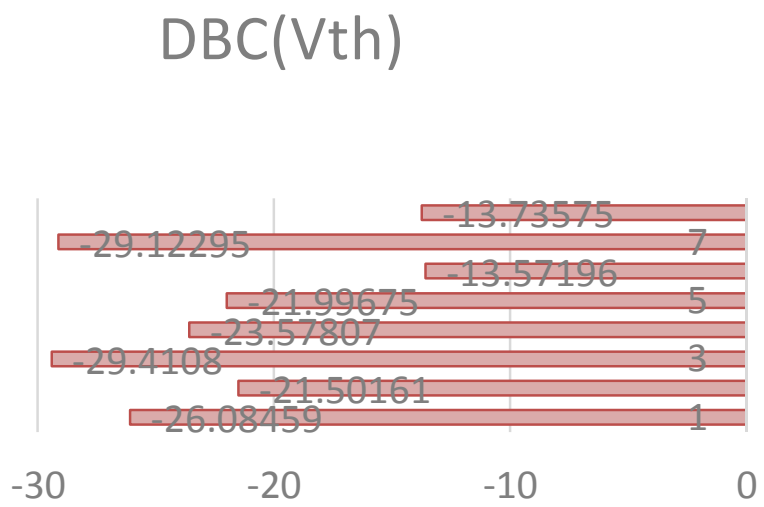

$\square$ Threshold

Figure S97. The threshold voltage data obtained for different devices made by using

DBC
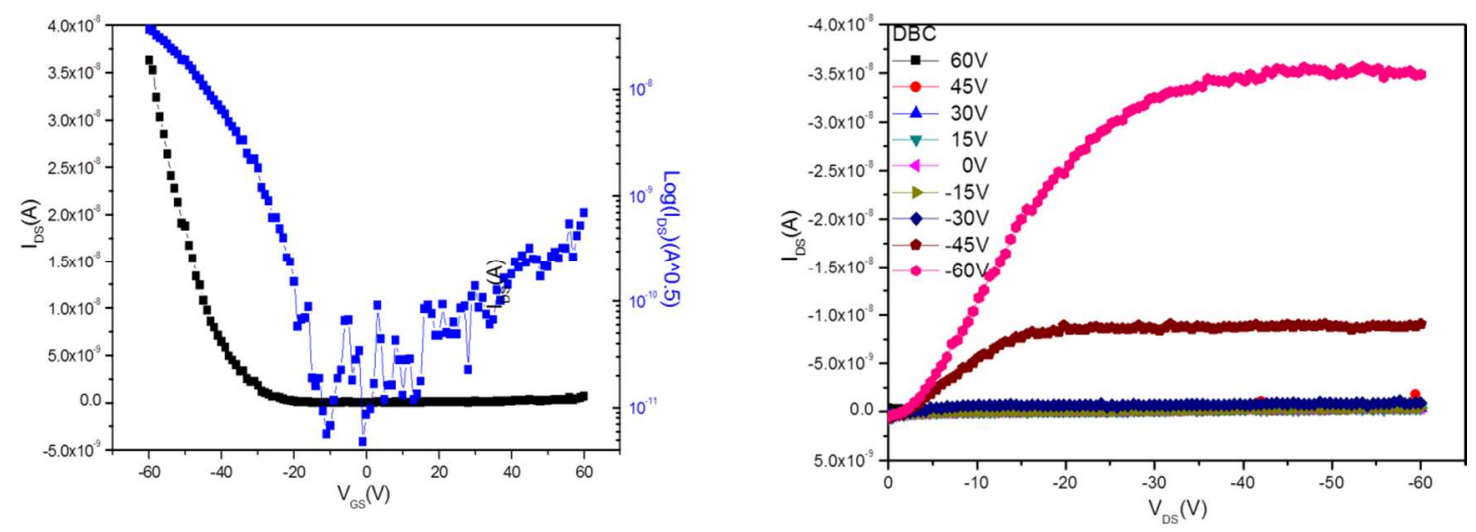

Figure $S 98 . I_{d} / V$ curve for DBC 
Table S1. Photo-physical data for coronene- and perylene-embedded derivatives

\begin{tabular}{|c|c|c|c|c|}
\hline Compd. & $\lambda_{\text {abs. }}, \mathrm{nm}$ & $\lambda_{\text {em., }}, \mathrm{nm}$ & $\begin{array}{c}\text { Stokes } \\
\text { shift } \\
\left(\mathrm{cm}^{-1}\right)\end{array}$ & $E_{\mathrm{g}}$ \\
\hline DBC & $\begin{array}{l}399,379, \\
330,293\end{array}$ & $\begin{array}{c}486,460 \\
(\max )\end{array}$ & 3324 & 3.061 \\
\hline DNC & $\begin{array}{c}392,358 \\
275\end{array}$ & $\begin{array}{c}520,500 \\
(\max )\end{array}$ & 5510 & 3.031 \\
\hline Met-DBC & $\begin{array}{c}408,387 \\
342,332, \\
281\end{array}$ & $\begin{array}{c}494,466 \\
(\max )\end{array}$ & 3051 & 2.988 \\
\hline BNP & $\begin{array}{l}444,421 \\
334,277\end{array}$ & 493 & 2239 & 2.707 \\
\hline NPP & $\begin{array}{c}428,406, \\
351,340, \\
276\end{array}$ & 482 & 2618 & 2.818 \\
\hline Met-BNP & $\begin{array}{l}436,411, \\
327,312,\end{array}$ & 463 & 1338 & 2.786 \\
\hline Flu-BNP & $\begin{array}{l}413,391, \\
303,281\end{array}$ & 483 & 3509 & 2.910 \\
\hline $\begin{array}{c}\text { Met- } \\
\text { PhBNP }\end{array}$ & $\begin{array}{c}450,419 \\
312\end{array}$ & 494 & 1979 & 2.684 \\
\hline Ph-BINP & $\begin{array}{l}479,421 \\
345,285\end{array}$ & $\begin{array}{c}536(\max ) \\
526\end{array}$ & 1865 & 2.520 \\
\hline Met-BFP & $\begin{array}{c}511,480 \\
447,358, \\
288\end{array}$ & 548 & 1321 & 2.362 \\
\hline
\end{tabular}

$E_{g}$, the optical band-gap

Table S2. Single-crystal OFET performance of DBC.

\begin{tabular}{ccccc}
\hline $\boldsymbol{\mu}$ & $\boldsymbol{\mu}_{\text {average }}$ & On/off & Threshold \\
& $\left(\mathrm{Cm}^{2} / \mathrm{Vs}\right)$ & $\left(\mathrm{Cm}^{2} / \mathrm{Vs}\right)$ & ratio & Voltage \\
& & & & $\left(\mathrm{V}_{\text {th }}, \mathrm{V}\right)$ \\
\hline DBC & $0.23-0.64$ & $0.42 \pm 0.15$ & $10^{3}-$ & $(-13)-(-29)$ \\
& & & $10^{4}$ & \\
\hline
\end{tabular}


Supporting Information

\section{Single-Crystal data of Ph-BINP, DNC, DBC and Flu-DBC}

Single-crystal data for Ph-BINP

Table 1. Crystal data and structure refinement for Ph-BINP.

Identification code

Empirical formula

Formula weight

i15298

Temperature

Wavelength

Crystal system

Space group

Unit cell dimensions

$\mathrm{C} 44 \mathrm{H} 26 \mathrm{O}$

570.65

$100.0(2) \mathrm{K}$

$0.71073 \AA$

Triclinic

$\mathrm{P}-1$
$\mathrm{a}=6.8964(6) \AA \quad \alpha=113.498(5)^{\circ}$
$\mathrm{b}=14.2616(13) \AA \quad \beta=97.210(5)^{\circ}$.
$\mathrm{c}=14.9139(13) \AA \quad \gamma=91.404(5)^{\circ}$.

Volume

Z

1330.1(2) $\AA^{3}$

2

Density (calculated)

$1.425 \mathrm{Mg} / \mathrm{m}^{3}$

Absorption coefficient

$0.083 \mathrm{~mm}^{-1}$

$\mathrm{F}(000)$

596

Crystal size

Theta range for data collection

$0.180 \times 0.140 \times 0.040 \mathrm{~mm}^{3}$

1.562 to $27.102^{\circ}$.

Index ranges

Reflections collected

Independent reflections

Completeness to theta $=25.000^{\circ}$

$-8<=\mathrm{h}<=8,-18<=\mathrm{k}<=18,-19<=\mathrm{l}<=19$

61961

$5862[\mathrm{R}(\mathrm{int})=0.1746]$

$100.0 \%$

Absorption correction

Semi-empirical from equivalents

Max. and min. transmission

0.9705 and 0.681

Refinement method

Full-matrix least-squares on $\mathrm{F}^{2}$

Data / restraints / parameters

5862 / 0 / 407

Goodness-of-fit on $\mathrm{F}^{2}$

1.020

Final $\mathrm{R}$ indices [I $>2$ sigma(I)]

$\mathrm{R} 1=0.0718, \mathrm{wR} 2=0.1727$

$\mathrm{R}$ indices (all data)

$\mathrm{R} 1=0.1436, \mathrm{wR} 2=0.2141$

$0.004(2)$

Largest diff. peak and hole

0.463 and -0.542 e. $\AA^{-3}$

Table 2. Atomic coordinates $\left(\times 10^{4}\right)$ and equivalent isotropic displacement parameters $\left(\AA^{2} \times 10^{3}\right)$ for $\mathrm{i} 15298$. $U(\mathrm{eq})$ is defined as one third of the trace of the orthogonalized $\mathrm{U}^{\mathrm{ij}}$ tensor.

\begin{tabular}{lrrrr}
\hline & $\mathrm{x}$ & $\mathrm{y}$ & $\mathrm{z}$ & $\mathrm{U}(\mathrm{eq})$ \\
\hline $\mathrm{C}(1)$ & & & $22(1)$ \\
$\mathrm{C}(2)$ & $7570(4)$ & $5556(2)$ & $6992(2)$ & $24(1)$ \\
$\mathrm{C}(3)$ & $7663(4)$ & $6625(2)$ & $7323(2)$ & $27(1)$ \\
$\mathrm{C}(4)$ & $7689(4)$ & $7126(3)$ & $6683(2)$ & $24(1)$ \\
$\mathrm{C}(5)$ & $7626(4)$ & $6557(2)$ & $5681(2)$ & $25(1)$ \\
$\mathrm{C}(6)$ & $7589(4)$ & $6764(2)$ & $4782(2)$ & $28(1)$ \\
$\mathrm{C}(7)$ & $7568(4)$ & $7675(3)$ & $4649(3)$ & $30(1)$ \\
$\mathrm{C}(8)$ & $7491(4)$ & $7624(3)$ & $3690(3)$ & $29(1)$ \\
$\mathrm{C}(9)$ & $7449(4)$ & $6699(3)$ & $2897(3)$ & $26(1)$ \\
$\mathrm{C}(10)$ & $7455(4)$ & $5780(3)$ & $3014(2)$ & $23(1)$ \\
$\mathrm{C}(11)$ & $7516(4)$ & $5808(2)$ & $3957(2)$ & $21(1)$ \\
$\mathrm{C}(12)$ & $7496(4)$ & $4989(2)$ & $4317(2)$ & $23(1)$ \\
$\mathrm{C}(13)$ & $7393(4)$ & $3944(2)$ & $3890(2)$ & $20(1)$ \\
$\mathrm{C}(14)$ & $7342(4)$ & $3365(2)$ & $4484(2)$ & $21(1)$ \\
$\mathrm{C}(15)$ & $7021(4)$ & $2268(2)$ & $4052(2)$ & $26(1)$ \\
$\mathrm{C}(16)$ & $6700(4)$ & $1696(2)$ & $3015(2)$ & $26(1)$ \\
$\mathrm{C}(17)$ & $6051(4)$ & $675(2)$ & $2595(2)$ & $25(1)$ \\
$\mathrm{C}(18)$ & $5651(4)$ & $177(2)$ & $3194(2)$ & $24(1)$ \\
$\mathrm{C}(19)$ & $6086(4)$ & $691(2)$ & $4201(2)$ & $22(1)$
\end{tabular}


Supporting Information

$\begin{array}{lrrrr}\mathrm{C}(20) & 7379(4) & 2266(2) & 5730(2) & 20(1) \\ \mathrm{C}(21) & 7933(4) & 1741(2) & 6380(2) & 21(1) \\ \mathrm{C}(22) & 8625(4) & 752(2) & 6024(2) & 23(1) \\ \mathrm{C}(23) & 9444(4) & 332(2) & 6652(2) & 25(1) \\ \mathrm{C}(24) & 9662(5) & 899(3) & 7668(2) & 31(1) \\ \mathrm{C}(25) & 8989(5) & 1852(3) & 8037(2) & 29(1) \\ \mathrm{C}(26) & 8075(4) & 2294(2) & 7415(2) & 21(1) \\ \mathrm{C}(27) & 7562(4) & 3353(2) & 7815(2) & 21(1) \\ \mathrm{C}(28) & 7168(4) & 3871(2) & 8803(2) & 24(1) \\ \mathrm{C}(29) & 7057(4) & 4923(2) & 9176(2) & 27(1) \\ \mathrm{C}(30) & 7256(4) & 5478(2) & 8613(2) & 25(1) \\ \mathrm{C}(31) & 7471(4) & 4989(2) & 7617(2) & 22(1) \\ \mathrm{C}(32) & 7552(4) & 3900(2) & 7202(2) & 21(1) \\ \mathrm{C}(33) & 7492(4) & 3329(2) & 6144(2) & 19(1) \\ \mathrm{C}(34) & 7443(4) & 3877(2) & 5519(2) & 19(1) \\ \mathrm{C}(35) & 7540(4) & 4967(2) & 5963(2) & 19(1) \\ \mathrm{C}(36) & 7560(4) & 5488(2) & 5353(2) & 22(1) \\ \mathrm{C}(37) & 6719(5) & 3323(3) & 9423(2) & 29(1) \\ \mathrm{C}(38) & 7725(5) & 3617(3) & 10374(2) & 31(1) \\ \mathrm{C}(39) & 7351(5) & 3090(3) & 10946(2) & 35(1) \\ \mathrm{C}(40) & 5897(5) & 2300(3) & 10604(3) & 38(1) \\ \mathrm{C}(41) & 4797(5) & 2022(3) & 9670(2) & 34(1) \\ \mathrm{C}(42) & 5215(5) & 2531(3) & 9090(2) & 31(1) \\ \text { O(1) } & 8280(4) & 56(2) & 443(2) & 63(1) \\ \mathrm{C}(43) & 9973(6) & 788(3) & 907(3) & 53(1) \\ \text { C(44) } & 8194(6) & -302(4) & -595(3) & 52(1) \\ & & & & \end{array}$

Table 3. Bond lengths $[\AA]$ and angles $\left[^{\circ}\right]$ for 115298 .

\begin{tabular}{ll}
\hline $\mathrm{C}(1)-\mathrm{C}(2)$ & $1.399(4)$ \\
$\mathrm{C}(1)-\mathrm{C}(35)$ & $1.423(4)$ \\
$\mathrm{C}(1)-\mathrm{C}(31)$ & $1.463(4)$ \\
$\mathrm{C}(2)-\mathrm{C}(3)$ & $1.404(4)$ \\
$\mathrm{C}(2)-\mathrm{H}(2)$ & 0.9500 \\
$\mathrm{C}(3)-\mathrm{C}(4)$ & $1.382(4)$ \\
$\mathrm{C}(3)-\mathrm{H}(3)$ & 0.9500 \\
$\mathrm{C}(4)-\mathrm{C}(36)$ & $1.401(4)$ \\
$\mathrm{C}(4)-\mathrm{C}(5)$ & $1.481(4)$ \\
$\mathrm{C}(5)-\mathrm{C}(6)$ & $1.390(4)$ \\
$\mathrm{C}(5)-\mathrm{C}(10)$ & $1.420(4)$ \\
$\mathrm{C}(6)-\mathrm{C}(7)$ & $1.397(5)$ \\
$\mathrm{C}(6)-\mathrm{H}(6)$ & 0.9500 \\
$\mathrm{C}(7)-\mathrm{C}(8)$ & $1.373(5)$ \\
$\mathrm{C}(7)-\mathrm{H}(7)$ & 0.9500 \\
$\mathrm{C}(8)-\mathrm{C}(9)$ & $1.389(4)$ \\
$\mathrm{C}(8)-\mathrm{H}(8)$ & 0.9500 \\
$\mathrm{C}(9)-\mathrm{C}(10)$ & $1.386(4)$ \\
$\mathrm{C}(9)-\mathrm{H}(9)$ & 0.9500 \\
$\mathrm{C}(10)-\mathrm{C}(11)$ & $1.468(4)$ \\
$\mathrm{C}(11)-\mathrm{C}(12)$ & $1.363(4)$ \\
$\mathrm{C}(11)-\mathrm{C}(36)$ & $1.413(4)$ \\
$\mathrm{C}(12)-\mathrm{C}(13)$ & $1.434(4)$ \\
$\mathrm{C}(12)-\mathrm{H}(12)$ & 0.9500 \\
$\mathrm{C}(13)-\mathrm{C}(34)$ & $1.412(4)$ \\
$\mathrm{C}(13)-\mathrm{C}(14)$ & $1.434(4)$ \\
$\mathrm{C}(14)-\mathrm{C}(15)$ & $1.417(4)$ \\
$\mathrm{C}(14)-\mathrm{C}(19)$ & $1.426(4)$ \\
$\mathrm{C}(15)-\mathrm{C}(16)$ & $1.372(4)$ \\
$\mathrm{C}(15)-\mathrm{H}(15)$ & 0.9500 \\
$\mathrm{C}(16)-\mathrm{C}(17)$ & $1.393(4)$ \\
$\mathrm{C}(16)-\mathrm{H}(16)$ & 0.9500 \\
&
\end{tabular}




\begin{tabular}{|c|c|}
\hline $\mathrm{C}(17)-\mathrm{C}(18)$ & $1.371(4)$ \\
\hline $\mathrm{C}(17)-\mathrm{H}(17)$ & 0.9500 \\
\hline C(18)-C(19) & $1.412(4)$ \\
\hline $\mathrm{C}(18)-\mathrm{H}(18)$ & 0.9500 \\
\hline C(19)-C(20) & $1.454(4)$ \\
\hline $\mathrm{C}(20)-\mathrm{C}(33)$ & $1.386(4)$ \\
\hline $\mathrm{C}(20)-\mathrm{C}(21)$ & $1.464(4)$ \\
\hline $\mathrm{C}(21)-\mathrm{C}(22)$ & $1.414(4)$ \\
\hline$C(21)-C(26)$ & $1.415(4)$ \\
\hline $\mathrm{C}(22)-\mathrm{C}(23)$ & $1.374(4)$ \\
\hline $\mathrm{C}(22)-\mathrm{H}(22)$ & 0.9500 \\
\hline $\mathrm{C}(23)-\mathrm{C}(24)$ & $1.389(4)$ \\
\hline $\mathrm{C}(23)-\mathrm{H}(23)$ & 0.9500 \\
\hline $\mathrm{C}(24)-\mathrm{C}(25)$ & $1.365(4)$ \\
\hline $\mathrm{C}(24)-\mathrm{H}(24)$ & 0.9500 \\
\hline$C(25)-C(26)$ & $1.412(4)$ \\
\hline $\mathrm{C}(25)-\mathrm{H}(25)$ & 0.9500 \\
\hline $\mathrm{C}(26)-\mathrm{C}(27)$ & $1.456(4)$ \\
\hline $\mathrm{C}(27)-\mathrm{C}(32)$ & $1.418(4)$ \\
\hline $\mathrm{C}(27)-\mathrm{C}(28)$ & $1.426(4)$ \\
\hline $\mathrm{C}(28)-\mathrm{C}(29)$ & $1.384(4)$ \\
\hline $\mathrm{C}(28)-\mathrm{C}(37)$ & $1.482(4)$ \\
\hline $\mathrm{C}(29)-\mathrm{C}(30)$ & $1.380(4)$ \\
\hline C(29)-H(29) & 0.9500 \\
\hline $\mathrm{C}(30)-\mathrm{C}(31)$ & $1.396(4)$ \\
\hline $\mathrm{C}(30)-\mathrm{H}(30)$ & 0.9500 \\
\hline $\mathrm{C}(31)-\mathrm{C}(32)$ & $1.430(4)$ \\
\hline$C(32)-C(33)$ & $1.454(4)$ \\
\hline $\mathrm{C}(33)-\mathrm{C}(34)$ & $1.433(4)$ \\
\hline $\mathrm{C}(34)-\mathrm{C}(35)$ & $1.422(4)$ \\
\hline$C(35)-C(36)$ & $1.386(4)$ \\
\hline $\mathrm{C}(37)-\mathrm{C}(38)$ & $1.390(4)$ \\
\hline$C(37)-C(42)$ & $1.402(5)$ \\
\hline $\mathrm{C}(38)-\mathrm{C}(39)$ & $1.384(5)$ \\
\hline $\mathrm{C}(38)-\mathrm{H}(38)$ & 0.9500 \\
\hline C(39)-C(40) & $1.376(5)$ \\
\hline C(39)-H(39) & 0.9500 \\
\hline $\mathrm{C}(40)-\mathrm{C}(41)$ & $1.397(5)$ \\
\hline $\mathrm{C}(40)-\mathrm{H}(40)$ & 0.9500 \\
\hline $\mathrm{C}(41)-\mathrm{C}(42)$ & $1.382(5)$ \\
\hline $\mathrm{C}(41)-\mathrm{H}(41)$ & 0.9500 \\
\hline $\mathrm{C}(42)-\mathrm{H}(42)$ & 0.9500 \\
\hline $\mathrm{O}(1)-\mathrm{C}(44)$ & $1.417(5)$ \\
\hline $\mathrm{O}(1)-\mathrm{C}(43)$ & $1.447(5)$ \\
\hline $\mathrm{C}(43)-\mathrm{C}(44) \# 1$ & $1.492(6)$ \\
\hline $\mathrm{C}(43)-\mathrm{H}(43 \mathrm{~A})$ & 0.9900 \\
\hline $\mathrm{C}(43)-\mathrm{H}(43 \mathrm{~B})$ & 0.9900 \\
\hline C(44)-C(43)\#1 & $1.492(6)$ \\
\hline $\mathrm{C}(44)-\mathrm{H}(44 \mathrm{~A})$ & 0.9900 \\
\hline $\mathrm{C}(44)-\mathrm{H}(44 \mathrm{~B})$ & 0.9900 \\
\hline $\mathrm{C}(2)-\mathrm{C}(1)-\mathrm{C}(35)$ & 117.7(3) \\
\hline $\mathrm{C}(2)-\mathrm{C}(1)-\mathrm{C}(31)$ & $125.3(3)$ \\
\hline $\mathrm{C}(35)-\mathrm{C}(1)-\mathrm{C}(31)$ & $117.0(3)$ \\
\hline $\mathrm{C}(1)-\mathrm{C}(2)-\mathrm{C}(3)$ & $122.8(3)$ \\
\hline $\mathrm{C}(1)-\mathrm{C}(2)-\mathrm{H}(2)$ & 118.6 \\
\hline $\mathrm{C}(3)-\mathrm{C}(2)-\mathrm{H}(2)$ & 118.6 \\
\hline$C(4)-C(3)-C(2)$ & $119.8(3)$ \\
\hline $\mathrm{C}(4)-\mathrm{C}(3)-\mathrm{H}(3)$ & 120.1 \\
\hline $\mathrm{C}(2)-\mathrm{C}(3)-\mathrm{H}(3)$ & 120.1 \\
\hline$C(3)-C(4)-C(36)$ & $117.3(3)$ \\
\hline $\mathrm{C}(3)-\mathrm{C}(4)-\mathrm{C}(5)$ & $137.0(3)$ \\
\hline
\end{tabular}




\begin{tabular}{|c|c|}
\hline$C(36)-C(4)-C(5)$ & $105.7(3)$ \\
\hline$C(6)-C(5)-C(10)$ & $120.4(3)$ \\
\hline$C(6)-C(5)-C(4)$ & $131.5(3)$ \\
\hline$C(10)-C(5)-C(4)$ & $108.0(3)$ \\
\hline $\mathrm{C}(5)-\mathrm{C}(6)-\mathrm{C}(7)$ & $118.4(3)$ \\
\hline $\mathrm{C}(5)-\mathrm{C}(6)-\mathrm{H}(6)$ & 120.8 \\
\hline $\mathrm{C}(7)-\mathrm{C}(6)-\mathrm{H}(6)$ & 120.8 \\
\hline $\mathrm{C}(8)-\mathrm{C}(7)-\mathrm{C}(6)$ & $120.9(3)$ \\
\hline $\mathrm{C}(8)-\mathrm{C}(7)-\mathrm{H}(7)$ & 119.5 \\
\hline $\mathrm{C}(6)-\mathrm{C}(7)-\mathrm{H}(7)$ & 119.5 \\
\hline $\mathrm{C}(7)-\mathrm{C}(8)-\mathrm{C}(9)$ & $121.5(3)$ \\
\hline $\mathrm{C}(7)-\mathrm{C}(8)-\mathrm{H}(8)$ & 119.2 \\
\hline $\mathrm{C}(9)-\mathrm{C}(8)-\mathrm{H}(8)$ & 119.2 \\
\hline $\mathrm{C}(10)-\mathrm{C}(9)-\mathrm{C}(8)$ & $118.7(3)$ \\
\hline $\mathrm{C}(10)-\mathrm{C}(9)-\mathrm{H}(9)$ & 120.7 \\
\hline $\mathrm{C}(8)-\mathrm{C}(9)-\mathrm{H}(9)$ & 120.7 \\
\hline $\mathrm{C}(9)-\mathrm{C}(10)-\mathrm{C}(5)$ & $120.1(3)$ \\
\hline$C(9)-C(10)-C(11)$ & $131.7(3)$ \\
\hline $\mathrm{C}(5)-\mathrm{C}(10)-\mathrm{C}(11)$ & $108.2(3)$ \\
\hline$C(12)-C(11)-C(36)$ & $118.9(3)$ \\
\hline$C(12)-C(11)-C(10)$ & $135.3(3)$ \\
\hline $\mathrm{C}(36)-\mathrm{C}(11)-\mathrm{C}(10)$ & $105.8(3)$ \\
\hline$C(11)-C(12)-C(13)$ & $120.3(3)$ \\
\hline $\mathrm{C}(11)-\mathrm{C}(12)-\mathrm{H}(12)$ & 119.9 \\
\hline $\mathrm{C}(13)-\mathrm{C}(12)-\mathrm{H}(12)$ & 119.9 \\
\hline $\mathrm{C}(34)-\mathrm{C}(13)-\mathrm{C}(14)$ & $117.8(3)$ \\
\hline$C(34)-C(13)-C(12)$ & $120.1(3)$ \\
\hline$C(14)-C(13)-C(12)$ & $121.9(3)$ \\
\hline$C(15)-C(14)-C(19)$ & $117.7(3)$ \\
\hline$C(15)-C(14)-C(13)$ & $121.9(3)$ \\
\hline$C(19)-C(14)-C(13)$ & $120.3(3)$ \\
\hline$C(16)-C(15)-C(14)$ & $122.1(3)$ \\
\hline $\mathrm{C}(16)-\mathrm{C}(15)-\mathrm{H}(15)$ & 119.0 \\
\hline $\mathrm{C}(14)-\mathrm{C}(15)-\mathrm{H}(15)$ & 119.0 \\
\hline$C(15)-C(16)-C(17)$ & $119.8(3)$ \\
\hline $\mathrm{C}(15)-\mathrm{C}(16)-\mathrm{H}(16)$ & 120.1 \\
\hline $\mathrm{C}(17)-\mathrm{C}(16)-\mathrm{H}(16)$ & 120.1 \\
\hline $\mathrm{C}(18)-\mathrm{C}(17)-\mathrm{C}(16)$ & $119.2(3)$ \\
\hline $\mathrm{C}(18)-\mathrm{C}(17)-\mathrm{H}(17)$ & 120.4 \\
\hline $\mathrm{C}(16)-\mathrm{C}(17)-\mathrm{H}(17)$ & 120.4 \\
\hline$C(17)-C(18)-C(19)$ & $122.7(3)$ \\
\hline $\mathrm{C}(17)-\mathrm{C}(18)-\mathrm{H}(18)$ & 118.6 \\
\hline $\mathrm{C}(19)-\mathrm{C}(18)-\mathrm{H}(18)$ & 118.6 \\
\hline $\mathrm{C}(18)-\mathrm{C}(19)-\mathrm{C}(14)$ & $117.5(3)$ \\
\hline $\mathrm{C}(18)-\mathrm{C}(19)-\mathrm{C}(20)$ & $123.1(3)$ \\
\hline$C(14)-C(19)-C(20)$ & $119.2(3)$ \\
\hline$C(33)-C(20)-C(19)$ & $118.9(3)$ \\
\hline $\mathrm{C}(33)-\mathrm{C}(20)-\mathrm{C}(21)$ & $118.2(3)$ \\
\hline$C(19)-C(20)-C(21)$ & $122.6(3)$ \\
\hline$C(22)-C(21)-C(26)$ & $118.1(3)$ \\
\hline$C(22)-C(21)-C(20)$ & $122.0(3)$ \\
\hline$C(26)-C(21)-C(20)$ & 119.1(3) \\
\hline $\mathrm{C}(23)-\mathrm{C}(22)-\mathrm{C}(21)$ & $121.8(3)$ \\
\hline $\mathrm{C}(23)-\mathrm{C}(22)-\mathrm{H}(22)$ & 119.1 \\
\hline $\mathrm{C}(21)-\mathrm{C}(22)-\mathrm{H}(22)$ & 119.1 \\
\hline $\mathrm{C}(22)-\mathrm{C}(23)-\mathrm{C}(24)$ & $119.7(3)$ \\
\hline $\mathrm{C}(22)-\mathrm{C}(23)-\mathrm{H}(23)$ & 120.2 \\
\hline $\mathrm{C}(24)-\mathrm{C}(23)-\mathrm{H}(23)$ & 120.2 \\
\hline$C(25)-C(24)-C(23)$ & $120.0(3)$ \\
\hline $\mathrm{C}(25)-\mathrm{C}(24)-\mathrm{H}(24)$ & 120.0 \\
\hline $\mathrm{C}(23)-\mathrm{C}(24)-\mathrm{H}(24)$ & 120.0 \\
\hline$C(24)-C(25)-C(26)$ & $122.0(3)$ \\
\hline
\end{tabular}




\begin{tabular}{|c|c|}
\hline $\mathrm{C}(24)-\mathrm{C}(25)-\mathrm{H}(25)$ & 119.0 \\
\hline $\mathrm{C}(26)-\mathrm{C}(25)-\mathrm{H}(25)$ & 119.0 \\
\hline$C(25)-C(26)-C(21)$ & $118.3(3)$ \\
\hline$C(25)-C(26)-C(27)$ & $121.2(3)$ \\
\hline $\mathrm{C}(21)-\mathrm{C}(26)-\mathrm{C}(27)$ & $119.8(3)$ \\
\hline $\mathrm{C}(32)-\mathrm{C}(27)-\mathrm{C}(28)$ & $119.0(3)$ \\
\hline $\mathrm{C}(32)-\mathrm{C}(27)-\mathrm{C}(26)$ & $117.9(3)$ \\
\hline$C(28)-C(27)-C(26)$ & $123.0(3)$ \\
\hline $\mathrm{C}(29)-\mathrm{C}(28)-\mathrm{C}(27)$ & $118.7(3)$ \\
\hline $\mathrm{C}(29)-\mathrm{C}(28)-\mathrm{C}(37)$ & $118.3(3)$ \\
\hline $\mathrm{C}(27)-\mathrm{C}(28)-\mathrm{C}(37)$ & $122.8(3)$ \\
\hline $\mathrm{C}(30)-\mathrm{C}(29)-\mathrm{C}(28)$ & $122.1(3)$ \\
\hline $\mathrm{C}(30)-\mathrm{C}(29)-\mathrm{H}(29)$ & 118.9 \\
\hline $\mathrm{C}(28)-\mathrm{C}(29)-\mathrm{H}(29)$ & 118.9 \\
\hline$C(29)-C(30)-C(31)$ & $121.0(3)$ \\
\hline $\mathrm{C}(29)-\mathrm{C}(30)-\mathrm{H}(30)$ & 119.5 \\
\hline $\mathrm{C}(31)-\mathrm{C}(30)-\mathrm{H}(30)$ & 119.5 \\
\hline$C(30)-C(31)-C(32)$ & $118.3(3)$ \\
\hline C(30)-C(31)-C(1) & $121.9(3)$ \\
\hline C(32)-C(31)-C(1) & $119.8(3)$ \\
\hline $\mathrm{C}(27)-\mathrm{C}(32)-\mathrm{C}(31)$ & $120.1(3)$ \\
\hline $\mathrm{C}(27)-\mathrm{C}(32)-\mathrm{C}(33)$ & $118.9(3)$ \\
\hline $\mathrm{C}(31)-\mathrm{C}(32)-\mathrm{C}(33)$ & $120.9(3)$ \\
\hline $\mathrm{C}(20)-\mathrm{C}(33)-\mathrm{C}(34)$ & $119.9(3)$ \\
\hline$C(20)-C(33)-C(32)$ & $120.9(3)$ \\
\hline $\mathrm{C}(34)-\mathrm{C}(33)-\mathrm{C}(32)$ & 119.1(3) \\
\hline $\mathrm{C}(13)-\mathrm{C}(34)-\mathrm{C}(35)$ & $119.5(3)$ \\
\hline $\mathrm{C}(13)-\mathrm{C}(34)-\mathrm{C}(33)$ & $121.8(3)$ \\
\hline$C(35)-C(34)-C(33)$ & $118.7(3)$ \\
\hline $\mathrm{C}(36)-\mathrm{C}(35)-\mathrm{C}(34)$ & $118.0(3)$ \\
\hline $\mathrm{C}(36)-\mathrm{C}(35)-\mathrm{C}(1)$ & $117.9(3)$ \\
\hline$C(34)-C(35)-C(1)$ & $124.0(3)$ \\
\hline C(35)-C(36)-C(4) & $124.5(3)$ \\
\hline$C(35)-C(36)-C(11)$ & $123.2(3)$ \\
\hline$C(4)-C(36)-C(11)$ & $112.3(3)$ \\
\hline $\mathrm{C}(38)-\mathrm{C}(37)-\mathrm{C}(42)$ & $118.1(3)$ \\
\hline $\mathrm{C}(38)-\mathrm{C}(37)-\mathrm{C}(28)$ & $120.5(3)$ \\
\hline $\mathrm{C}(42)-\mathrm{C}(37)-\mathrm{C}(28)$ & $121.3(3)$ \\
\hline $\mathrm{C}(39)-\mathrm{C}(38)-\mathrm{C}(37)$ & $120.7(3)$ \\
\hline $\mathrm{C}(39)-\mathrm{C}(38)-\mathrm{H}(38)$ & 119.6 \\
\hline $\mathrm{C}(37)-\mathrm{C}(38)-\mathrm{H}(38)$ & 119.6 \\
\hline $\mathrm{C}(40)-\mathrm{C}(39)-\mathrm{C}(38)$ & $120.5(3)$ \\
\hline $\mathrm{C}(40)-\mathrm{C}(39)-\mathrm{H}(39)$ & 119.7 \\
\hline $\mathrm{C}(38)-\mathrm{C}(39)-\mathrm{H}(39)$ & 119.7 \\
\hline$C(39)-C(40)-C(41)$ & $119.8(3)$ \\
\hline $\mathrm{C}(39)-\mathrm{C}(40)-\mathrm{H}(40)$ & 120.1 \\
\hline $\mathrm{C}(41)-\mathrm{C}(40)-\mathrm{H}(40)$ & 120.1 \\
\hline $\mathrm{C}(42)-\mathrm{C}(41)-\mathrm{C}(40)$ & $119.5(3)$ \\
\hline $\mathrm{C}(42)-\mathrm{C}(41)-\mathrm{H}(41)$ & 120.3 \\
\hline $\mathrm{C}(40)-\mathrm{C}(41)-\mathrm{H}(41)$ & 120.3 \\
\hline$C(41)-C(42)-C(37)$ & $121.2(3)$ \\
\hline $\mathrm{C}(41)-\mathrm{C}(42)-\mathrm{H}(42)$ & 119.4 \\
\hline $\mathrm{C}(37)-\mathrm{C}(42)-\mathrm{H}(42)$ & 119.4 \\
\hline $\mathrm{C}(44)-\mathrm{O}(1)-\mathrm{C}(43)$ & $108.3(3)$ \\
\hline $\mathrm{O}(1)-\mathrm{C}(43)-\mathrm{C}(44) \# 1$ & $110.7(3)$ \\
\hline $\mathrm{O}(1)-\mathrm{C}(43)-\mathrm{H}(43 \mathrm{~A})$ & 109.5 \\
\hline $\mathrm{C}(44) \# 1-\mathrm{C}(43)-\mathrm{H}(43 \mathrm{~A})$ & 109.5 \\
\hline $\mathrm{O}(1)-\mathrm{C}(43)-\mathrm{H}(43 \mathrm{~B})$ & 109.5 \\
\hline $\mathrm{C}(44) \# 1-\mathrm{C}(43)-\mathrm{H}(43 \mathrm{~B})$ & 109.5 \\
\hline $\mathrm{H}(43 \mathrm{~A})-\mathrm{C}(43)-\mathrm{H}(43 \mathrm{~B})$ & 108.1 \\
\hline $\mathrm{O}(1)-\mathrm{C}(44)-\mathrm{C}(43) \# 1$ & $110.3(3)$ \\
\hline $\mathrm{O}(1)-\mathrm{C}(44)-\mathrm{H}(44 \mathrm{~A})$ & 109.6 \\
\hline
\end{tabular}




$\begin{array}{ll}\mathrm{C}(43) \# 1-\mathrm{C}(44)-\mathrm{H}(44 \mathrm{~A}) & 109.6 \\ \mathrm{O}(1)-\mathrm{C}(44)-\mathrm{H}(44 \mathrm{~B}) & 109.6 \\ \mathrm{C}(43) \# 1-\mathrm{C}(44)-\mathrm{H}(44 \mathrm{~B}) & 109.6 \\ \mathrm{H}(44 \mathrm{~A})-\mathrm{C}(44)-\mathrm{H}(44 \mathrm{~B}) & 108.1\end{array}$

Symmetry transformations used to generate equivalent atoms:

$\# 1-x+2,-y,-z$

Table 4. Anisotropic displacement parameters $\left(\AA^{2} \times 10^{3}\right)$ for i15298. The anisotropic displacement factor exponent takes the form: $-2 \pi^{2}\left[h^{2} a * 2 U^{11}+\ldots+2 h k a^{*} b^{*} U^{12}\right]$

\begin{tabular}{|c|c|c|c|c|c|c|}
\hline & $\mathrm{U}^{11}$ & $\mathrm{U}^{22}$ & $\mathrm{U}^{33}$ & $\mathrm{U}^{23}$ & $\mathrm{U}^{13}$ & $\mathrm{U}^{12}$ \\
\hline$\overline{C(1)}$ & $19(1)$ & $23(2)$ & $22(2)$ & $8(1)$ & $4(1)$ & $4(1)$ \\
\hline $\mathrm{C}(2)$ & $24(2)$ & $21(2)$ & $22(2)$ & $3(1)$ & $4(1)$ & $3(1)$ \\
\hline$C(3)$ & $22(2)$ & $23(2)$ & $32(2)$ & $8(2)$ & $4(1)$ & 1(1) \\
\hline C(4) & $15(1)$ & $23(2)$ & $34(2)$ & $13(2)$ & $2(1)$ & 1(1) \\
\hline$C(5)$ & $14(1)$ & $29(2)$ & $33(2)$ & $15(2)$ & $3(1)$ & 1(1) \\
\hline$C(6)$ & $18(2)$ & $25(2)$ & $40(2)$ & $13(2)$ & $5(1)$ & $2(1)$ \\
\hline$C(7)$ & $22(2)$ & $31(2)$ & $42(2)$ & $21(2)$ & $1(2)$ & 1(1) \\
\hline $\mathrm{C}(8)$ & $21(2)$ & $39(2)$ & $34(2)$ & $23(2)$ & 1(1) & $2(1)$ \\
\hline C(9) & $19(2)$ & $33(2)$ & $32(2)$ & $18(2)$ & $5(1)$ & $4(1)$ \\
\hline$C(10)$ & $15(1)$ & $28(2)$ & $29(2)$ & $15(2)$ & $2(1)$ & $3(1)$ \\
\hline $\mathrm{C}(11)$ & $15(1)$ & $25(2)$ & $24(2)$ & $11(2)$ & $3(1)$ & $3(1)$ \\
\hline $\mathrm{C}(12)$ & $16(1)$ & $33(2)$ & $19(2)$ & $12(2)$ & 2(1) & 2(1) \\
\hline $\mathrm{C}(13)$ & $17(1)$ & $24(2)$ & $19(2)$ & $7(1)$ & $4(1)$ & $2(1)$ \\
\hline $\mathrm{C}(14)$ & $17(1)$ & $25(2)$ & $22(2)$ & $9(1)$ & $4(1)$ & $4(1)$ \\
\hline$C(15)$ & $28(2)$ & $31(2)$ & $20(2)$ & $11(2)$ & $3(1)$ & $7(1)$ \\
\hline$C(16)$ & $31(2)$ & $23(2)$ & $16(2)$ & $1(1)$ & 1(1) & $6(1)$ \\
\hline $\mathrm{C}(17)$ & $24(2)$ & $23(2)$ & $24(2)$ & $4(2)$ & 1(1) & 1(1) \\
\hline $\mathrm{C}(18)$ & $24(2)$ & $24(2)$ & $24(2)$ & $10(2)$ & $7(1)$ & $6(1)$ \\
\hline$C(19)$ & $18(1)$ & $25(2)$ & $21(2)$ & $7(1)$ & $5(1)$ & $5(1)$ \\
\hline $\mathrm{C}(20)$ & $17(1)$ & $22(2)$ & $20(2)$ & $7(1)$ & $5(1)$ & $3(1)$ \\
\hline $\mathrm{C}(21)$ & $19(2)$ & $24(2)$ & $19(2)$ & $7(1)$ & $4(1)$ & 1(1) \\
\hline $\mathrm{C}(22)$ & $21(2)$ & $25(2)$ & $22(2)$ & $9(1)$ & $5(1)$ & 2(1) \\
\hline $\mathrm{C}(23)$ & $25(2)$ & $24(2)$ & $30(2)$ & $12(2)$ & $8(1)$ & $7(1)$ \\
\hline $\mathrm{C}(24)$ & $34(2)$ & $37(2)$ & $27(2)$ & $17(2)$ & $5(1)$ & $13(2)$ \\
\hline$C(25)$ & $33(2)$ & $34(2)$ & $22(2)$ & $12(2)$ & $7(1)$ & $10(2)$ \\
\hline$C(26)$ & $19(1)$ & $26(2)$ & $19(2)$ & $10(1)$ & $3(1)$ & $2(1)$ \\
\hline $\mathrm{C}(27)$ & $15(1)$ & $25(2)$ & $23(2)$ & $9(1)$ & $-1(1)$ & $0(1)$ \\
\hline $\mathrm{C}(28)$ & $22(2)$ & $29(2)$ & $18(2)$ & $7(1)$ & $1(1)$ & $4(1)$ \\
\hline $\mathrm{C}(29)$ & $28(2)$ & $29(2)$ & $19(2)$ & $5(2)$ & $3(1)$ & 1(1) \\
\hline $\mathrm{C}(30)$ & $25(2)$ & $20(2)$ & $24(2)$ & $4(1)$ & $3(1)$ & $2(1)$ \\
\hline$C(31)$ & $15(1)$ & $28(2)$ & $20(2)$ & $7(1)$ & 1(1) & $4(1)$ \\
\hline$C(32)$ & $15(1)$ & $24(2)$ & $20(2)$ & $5(1)$ & $2(1)$ & $3(1)$ \\
\hline $\mathrm{C}(33)$ & $13(1)$ & $23(2)$ & $18(2)$ & $7(1)$ & 2(1) & 1(1) \\
\hline $\mathrm{C}(34)$ & $14(1)$ & $22(2)$ & $21(2)$ & $9(1)$ & $2(1)$ & $-1(1)$ \\
\hline$C(35)$ & $13(1)$ & $23(2)$ & $20(2)$ & $7(1)$ & 1(1) & $0(1)$ \\
\hline$C(36)$ & $15(1)$ & $22(2)$ & $26(2)$ & $9(1)$ & $1(1)$ & $-1(1)$ \\
\hline$C(37)$ & $36(2)$ & $28(2)$ & $18(2)$ & $5(2)$ & $5(1)$ & $6(2)$ \\
\hline $\mathrm{C}(38)$ & $31(2)$ & $33(2)$ & $25(2)$ & $8(2)$ & $2(1)$ & $2(2)$ \\
\hline C(39) & $43(2)$ & $40(2)$ & $21(2)$ & $10(2)$ & $4(2)$ & $9(2)$ \\
\hline $\mathrm{C}(40)$ & $49(2)$ & $40(2)$ & $31(2)$ & $19(2)$ & $11(2)$ & $8(2)$ \\
\hline $\mathrm{C}(41)$ & $39(2)$ & $32(2)$ & $31(2)$ & $12(2)$ & $6(2)$ & $5(2)$ \\
\hline$C(42)$ & $34(2)$ & $32(2)$ & $23(2)$ & $8(2)$ & $2(1)$ & $8(2)$ \\
\hline $\mathrm{O}(1)$ & $56(2)$ & $76(2)$ & $57(2)$ & $29(2)$ & $10(2)$ & $-1(2)$ \\
\hline$C(43)$ & $63(3)$ & $40(2)$ & $42(2)$ & $4(2)$ & $2(2)$ & $1(2)$ \\
\hline$C(44)$ & $44(2)$ & $72(3)$ & $39(2)$ & $27(2)$ & $-8(2)$ & $-14(2)$ \\
\hline
\end{tabular}




\section{Single-crystal data for DNC}

Table 1. Crystal data and structure refinement for DNC.

Identification code

Empirical formula

i15472

Formula weight

$\mathrm{C} 20 \mathrm{H} 10$

Temperature

Wavelength

250.28

$100.0(2) \mathrm{K}$

Crystal system

Space group

Unit cell dimensions

Monoclinic

$\mathrm{C} 2 / \mathrm{c}$

$\mathrm{a}=25.1911(10) \AA \quad \alpha=90^{\circ}$.

$\mathrm{b}=12.5917(5) \AA \quad \beta=94.052(2)^{\circ}$.

$\mathrm{c}=7.2168(3) \AA \quad \gamma=90^{\circ}$.

Volume

Z

2283.44(16) $\AA^{3}$

Density (calculated)

Absorption coefficient

$1.456 \mathrm{Mg} / \mathrm{m}^{3}$

$\mathrm{F}(000)$

Crystal size

Theta range for data collection

$0.083 \mathrm{~mm}^{-1}$

1040

Index ranges

Reflections collected

Independent reflections

Completeness to theta $=25.000^{\circ}$

$0.220 \times 0.100 \times 0.060 \mathrm{~mm}^{3}$

1.621 to $27.101^{\circ}$.

$-32<=\mathrm{h}<=32,-16<=\mathrm{k}<=16,-9<=\mathrm{k}<=9$

42938

$2526[\mathrm{R}($ int $)=0.0594]$

$100.0 \%$

Semi-empirical from equivalents

Absorption correction

0.9705 and 0.9104

Refinement method

Data / restraints / parameters

Full-matrix least-squares on $\mathrm{F}^{2}$

Goodness-of-fit on $\mathrm{F}^{2}$

2526 / 0 / 184

Final $\mathrm{R}$ indices [I $>2 \operatorname{sigma}(\mathrm{I})]$

1.294

$\mathrm{R}$ indices (all data)

$\mathrm{R} 1=0.0543, \mathrm{wR} 2=0.1845$

$\mathrm{R} 1=0.0594, \mathrm{wR} 2=0.1877$

Extinction coefficient

$0.0040(8)$

Largest diff. peak and hole

0.559 and -0.384 e. $\AA^{-3}$

Table 2. Atomic coordinates $\left(\times 10^{4}\right)$ and equivalent isotropic displacement parameters $\left(\AA^{2} \times 10^{3}\right)$ for $\mathrm{i} 15472$. $U(\mathrm{eq})$ is defined as one third of the trace of the orthogonalized $\mathrm{U}^{\mathrm{ij}}$ tensor.

\begin{tabular}{llrrr}
\hline & $\mathrm{x}$ & $\mathrm{y}$ & $\mathrm{z}$ & $\mathrm{U}(\mathrm{eq})$ \\
\hline $\mathrm{C}(1)$ & 5000 & $2564(2)$ & 2500 & $11(1)$ \\
$\mathrm{C}(2)$ & $4537(1)$ & $2007(2)$ & $3081(3)$ & $12(1)$ \\
$\mathrm{C}(3)$ & $4567(1)$ & $935(2)$ & $3749(3)$ & $13(1)$ \\
$\mathrm{C}(4)$ & $4132(1)$ & $408(2)$ & $4276(3)$ & $14(1)$ \\
$\mathrm{C}(5)$ & $3615(1)$ & $858(2)$ & $3951(3)$ & $14(1)$ \\
$\mathrm{C}(6)$ & $3153(1)$ & $245(2)$ & $4159(3)$ & $18(1)$ \\
$\mathrm{C}(7)$ & $2658(1)$ & $647(2)$ & $3658(3)$ & $20(1)$ \\
$\mathrm{C}(8)$ & $2610(1)$ & $1673(2)$ & $2910(3)$ & $19(1)$ \\
$\mathrm{C}(9)$ & $3054(1)$ & $2298(2)$ & $2748(3)$ & $16(1)$ \\
$\mathrm{C}(10)$ & $3570(1)$ & $1925(2)$ & $3331(3)$ & $13(1)$ \\
$\mathrm{C}(11)$ & $4052(1)$ & $2555(2)$ & $3196(3)$ & $12(1)$ \\
$\mathrm{C}(12)$ & $4058(1)$ & $3694(2)$ & $3276(3)$ & $12(1)$ \\
$\mathrm{C}(13)$ & $3626(1)$ & $4298(2)$ & $3910(3)$ & $13(1)$ \\
$\mathrm{C}(14)$ & $3638(1)$ & $5378(2)$ & $3980(3)$ & $13(1)$ \\
$\mathrm{C}(15)$ & $4083(1)$ & $5955(2)$ & $3431(3)$ & $13(1)$ \\
$\mathrm{C}(16)$ & $4093(1)$ & $7083(2)$ & $3404(3)$ & $15(1)$ \\
$\mathrm{C}(17)$ & $4534(1)$ & $7619(2)$ & $2946(3)$ & $13(1)$ \\
$\mathrm{C}(18)$ & 5000 & $7068(2)$ & 2500 & $12(1)$ \\
$\mathrm{C}(19)$ & 5000 & $5944(2)$ & 2500 &
\end{tabular}


Supporting Information

$\begin{array}{lllll}\mathrm{C}(20) & 4537(1) & 5380(2) & 2959(3) & 12(1) \\ \mathrm{C}(21) & 4530(1) & 4249(2) & 2926(3) & 11(1) \\ \mathrm{C}(22) & 5000 & 3675(2) & 2500 & 11(1)\end{array}$

Table 3. Bond lengths $[\AA]$ and angles $\left[{ }^{\circ}\right]$ for $i 15472$.

\begin{tabular}{|c|c|}
\hline $\mathrm{C}(1)-\mathrm{C}(22)$ & $1.399(4)$ \\
\hline $\mathrm{C}(1)-\mathrm{C}(2) \# 1$ & $1.448(2)$ \\
\hline $\mathrm{C}(1)-\mathrm{C}(2)$ & $1.448(2)$ \\
\hline$C(2)-C(11)$ & $1.412(3)$ \\
\hline $\mathrm{C}(2)-\mathrm{C}(3)$ & $1.434(3)$ \\
\hline$C(3)-C(4)$ & $1.357(3)$ \\
\hline $\mathrm{C}(3)-\mathrm{H}(3)$ & 0.9500 \\
\hline$C(4)-C(5)$ & $1.424(3)$ \\
\hline $\mathrm{C}(4)-\mathrm{H}(4)$ & 0.9500 \\
\hline$C(5)-C(6)$ & $1.414(3)$ \\
\hline$C(5)-C(10)$ & $1.418(3)$ \\
\hline $\mathrm{C}(6)-\mathrm{C}(7)$ & $1.370(3)$ \\
\hline $\mathrm{C}(6)-\mathrm{H}(6)$ & 0.9500 \\
\hline$C(7)-C(8)$ & $1.402(3)$ \\
\hline $\mathrm{C}(7)-\mathrm{H}(7)$ & 0.9500 \\
\hline $\mathrm{C}(8)-\mathrm{C}(9)$ & $1.379(3)$ \\
\hline $\mathrm{C}(8)-\mathrm{H}(8)$ & 0.9500 \\
\hline $\mathrm{C}(9)-\mathrm{C}(10)$ & $1.417(3)$ \\
\hline $\mathrm{C}(9)-\mathrm{H}(9)$ & 0.9500 \\
\hline C(10)-C(11) & $1.458(3)$ \\
\hline$C(11)-C(12)$ & $1.436(3)$ \\
\hline$C(12)-C(21)$ & $1.418(3)$ \\
\hline $\mathrm{C}(12)-\mathrm{C}(13)$ & $1.429(3)$ \\
\hline C(13)-C(14) & $1.361(3)$ \\
\hline C(13)-H(13) & 0.9500 \\
\hline$C(14)-C(15)$ & $1.415(3)$ \\
\hline C(14)-H(14) & 0.9500 \\
\hline$C(15)-C(20)$ & $1.415(3)$ \\
\hline$C(15)-C(16)$ & $1.422(3)$ \\
\hline C(16)-C(17) & $1.360(3)$ \\
\hline $\mathrm{C}(16)-\mathrm{H}(16)$ & 0.9500 \\
\hline C(17)-C(18) & $1.420(2)$ \\
\hline $\mathrm{C}(17)-\mathrm{H}(17)$ & 0.9500 \\
\hline C(18)-C(19) & $1.415(4)$ \\
\hline C(18)-C(17)\#1 & $1.420(2)$ \\
\hline$C(19)-C(20)$ & $1.425(2)$ \\
\hline C(19)-C(20)\#1 & $1.425(2)$ \\
\hline$C(20)-C(21)$ & $1.425(3)$ \\
\hline $\mathrm{C}(21)-\mathrm{C}(22)$ & $1.438(2)$ \\
\hline $\mathrm{C}(22)-\mathrm{C}(21) \# 1$ & $1.438(2)$ \\
\hline $\mathrm{C}(22)-\mathrm{C}(1)-\mathrm{C}(2) \# 1$ & $118.99(12)$ \\
\hline $\mathrm{C}(22)-\mathrm{C}(1)-\mathrm{C}(2)$ & $118.99(12)$ \\
\hline $\mathrm{C}(2) \# 1-\mathrm{C}(1)-\mathrm{C}(2)$ & $122.0(2)$ \\
\hline $\mathrm{C}(11)-\mathrm{C}(2)-\mathrm{C}(3)$ & $117.67(17)$ \\
\hline$C(11)-C(2)-C(1)$ & $119.96(19)$ \\
\hline $\mathrm{C}(3)-\mathrm{C}(2)-\mathrm{C}(1)$ & $122.00(18)$ \\
\hline$C(4)-C(3)-C(2)$ & $122.07(18)$ \\
\hline $\mathrm{C}(4)-\mathrm{C}(3)-\mathrm{H}(3)$ & 119.0 \\
\hline $\mathrm{C}(2)-\mathrm{C}(3)-\mathrm{H}(3)$ & 119.0 \\
\hline$C(3)-C(4)-C(5)$ & $120.36(19)$ \\
\hline $\mathrm{C}(3)-\mathrm{C}(4)-\mathrm{H}(4)$ & 119.8 \\
\hline $\mathrm{C}(5)-\mathrm{C}(4)-\mathrm{H}(4)$ & 119.8 \\
\hline $\mathrm{C}(6)-\mathrm{C}(5)-\mathrm{C}(10)$ & $120.11(19)$ \\
\hline
\end{tabular}




\begin{tabular}{|c|c|}
\hline$C(6)-C(5)-C(4)$ & $121.04(19)$ \\
\hline$C(10)-C(5)-C(4)$ & $118.78(18)$ \\
\hline$C(7)-C(6)-C(5)$ & $120.8(2)$ \\
\hline $\mathrm{C}(7)-\mathrm{C}(6)-\mathrm{H}(6)$ & 119.6 \\
\hline $\mathrm{C}(5)-\mathrm{C}(6)-\mathrm{H}(6)$ & 119.6 \\
\hline $\mathrm{C}(6)-\mathrm{C}(7)-\mathrm{C}(8)$ & $119.58(19)$ \\
\hline $\mathrm{C}(6)-\mathrm{C}(7)-\mathrm{H}(7)$ & 120.2 \\
\hline $\mathrm{C}(8)-\mathrm{C}(7)-\mathrm{H}(7)$ & 120.2 \\
\hline $\mathrm{C}(9)-\mathrm{C}(8)-\mathrm{C}(7)$ & $120.7(2)$ \\
\hline $\mathrm{C}(9)-\mathrm{C}(8)-\mathrm{H}(8)$ & 119.7 \\
\hline $\mathrm{C}(7)-\mathrm{C}(8)-\mathrm{H}(8)$ & 119.7 \\
\hline C(8)-C(9)-C(10) & $121.14(19)$ \\
\hline C(8)-C(9)-H(9) & 119.4 \\
\hline $\mathrm{C}(10)-\mathrm{C}(9)-\mathrm{H}(9)$ & 119.4 \\
\hline $\mathrm{C}(9)-\mathrm{C}(10)-\mathrm{C}(5)$ & $117.37(18)$ \\
\hline $\mathrm{C}(9)-\mathrm{C}(10)-\mathrm{C}(11)$ & $123.35(18)$ \\
\hline$C(5)-C(10)-C(11)$ & $119.13(18)$ \\
\hline$C(2)-C(11)-C(12)$ & $118.89(17)$ \\
\hline $\mathrm{C}(2)-\mathrm{C}(11)-\mathrm{C}(10)$ & $117.85(18)$ \\
\hline $\mathrm{C}(12)-\mathrm{C}(11)-\mathrm{C}(10)$ & $123.17(17)$ \\
\hline$C(21)-C(12)-C(13)$ & $117.57(18)$ \\
\hline $\mathrm{C}(21)-\mathrm{C}(12)-\mathrm{C}(11)$ & $119.47(17)$ \\
\hline $\mathrm{C}(13)-\mathrm{C}(12)-\mathrm{C}(11)$ & $122.54(18)$ \\
\hline$C(14)-C(13)-C(12)$ & $121.83(18)$ \\
\hline $\mathrm{C}(14)-\mathrm{C}(13)-\mathrm{H}(13)$ & 119.1 \\
\hline $\mathrm{C}(12)-\mathrm{C}(13)-\mathrm{H}(13)$ & 119.1 \\
\hline$C(13)-C(14)-C(15)$ & $121.25(18)$ \\
\hline $\mathrm{C}(13)-\mathrm{C}(14)-\mathrm{H}(14)$ & 119.4 \\
\hline $\mathrm{C}(15)-\mathrm{C}(14)-\mathrm{H}(14)$ & 119.4 \\
\hline$C(20)-C(15)-C(14)$ & $118.31(18)$ \\
\hline $\mathrm{C}(20)-\mathrm{C}(15)-\mathrm{C}(16)$ & $119.48(18)$ \\
\hline$C(14)-C(15)-C(16)$ & $122.17(18)$ \\
\hline$C(17)-C(16)-C(15)$ & $121.01(18)$ \\
\hline $\mathrm{C}(17)-\mathrm{C}(16)-\mathrm{H}(16)$ & 119.5 \\
\hline $\mathrm{C}(15)-\mathrm{C}(16)-\mathrm{H}(16)$ & 119.5 \\
\hline $\mathrm{C}(16)-\mathrm{C}(17)-\mathrm{C}(18)$ & $121.0(2)$ \\
\hline $\mathrm{C}(16)-\mathrm{C}(17)-\mathrm{H}(17)$ & 119.5 \\
\hline C(18)-C(17)-H(17) & 119.5 \\
\hline$C(19)-C(18)-C(17) \# 1$ & $119.24(13)$ \\
\hline C(19)-C(18)-C(17) & $119.24(13)$ \\
\hline $\mathrm{C}(17) \# 1-\mathrm{C}(18)-\mathrm{C}(17)$ & $121.5(3)$ \\
\hline $\mathrm{C}(18)-\mathrm{C}(19)-\mathrm{C}(20)$ & $119.90(13)$ \\
\hline $\mathrm{C}(18)-\mathrm{C}(19)-\mathrm{C}(20) \# 1$ & $119.90(13)$ \\
\hline$C(20)-C(19)-C(20) \# 1$ & $120.2(3)$ \\
\hline$C(15)-C(20)-C(21)$ & $120.46(17)$ \\
\hline$C(15)-C(20)-C(19)$ & $119.34(19)$ \\
\hline$C(21)-C(20)-C(19)$ & $120.19(18)$ \\
\hline $\mathrm{C}(12)-\mathrm{C}(21)-\mathrm{C}(20)$ & $119.88(17)$ \\
\hline$C(12)-C(21)-C(22)$ & $120.30(19)$ \\
\hline $\mathrm{C}(20)-\mathrm{C}(21)-\mathrm{C}(22)$ & $119.81(18)$ \\
\hline $\mathrm{C}(1)-\mathrm{C}(22)-\mathrm{C}(21)$ & $120.14(12)$ \\
\hline $\mathrm{C}(1)-\mathrm{C}(22)-\mathrm{C}(21) \# 1$ & $120.14(12)$ \\
\hline $\mathrm{C}(21)-\mathrm{C}(22)-\mathrm{C}(21) \# 1$ & $119.7(2)$ \\
\hline
\end{tabular}

Symmetry transformations used to generate equivalent atoms:

$\# 1-\mathrm{x}+1, \mathrm{y},-\mathrm{z}+1 / 2$

Table 4. Anisotropic displacement parameters $\left(\AA^{2} \times 10^{3}\right)$ for i15472. The anisotropic displacement factor exponent takes the form: $-2 \pi^{2}\left[h^{2} a^{* 2} U^{11}+\ldots+2 h k a^{*} b^{*} U^{12}\right]$

$\begin{array}{lllll}\mathrm{U}^{11} & \mathrm{U}^{22} & \mathrm{U}^{33} & \mathrm{U}^{23} & \mathrm{U}^{13}\end{array}$


Supporting Information

\begin{tabular}{llllrrr}
\hline $\mathrm{C}(1)$ & $10(1)$ & $14(1)$ & $7(1)$ & 0 & $0(1)$ & 0 \\
$\mathrm{C}(2)$ & $12(1)$ & $13(1)$ & $10(1)$ & $-2(1)$ & $1(1)$ & $-1(1)$ \\
$\mathrm{C}(3)$ & $12(1)$ & $14(1)$ & $13(1)$ & $-1(1)$ & $2(1)$ & $2(1)$ \\
$\mathrm{C}(4)$ & $18(1)$ & $12(1)$ & $14(1)$ & $0(1)$ & $2(1)$ & $0(1)$ \\
$\mathrm{C}(5)$ & $14(1)$ & $16(1)$ & $13(1)$ & $-1(1)$ & $4(1)$ & $-2(1)$ \\
$\mathrm{C}(6)$ & $19(1)$ & $16(1)$ & $20(1)$ & $0(1)$ & $6(1)$ & $-4(1)$ \\
$\mathrm{C}(7)$ & $16(1)$ & $22(1)$ & $24(1)$ & $-4(1)$ & $6(1)$ & $-6(1)$ \\
$\mathrm{C}(8)$ & $11(1)$ & $24(1)$ & $23(1)$ & $-4(1)$ & $1(1)$ & $-1(1)$ \\
$\mathrm{C}(9)$ & $14(1)$ & $16(1)$ & $17(1)$ & $-1(1)$ & $2(1)$ & $0(1)$ \\
$\mathrm{C}(10)$ & $12(1)$ & $15(1)$ & $14(1)$ & $-2(1)$ & $3(1)$ & $-1(1)$ \\
$\mathrm{C}(11)$ & $11(1)$ & $14(1)$ & $10(1)$ & $1(1)$ & $1(1)$ & $0(1)$ \\
$\mathrm{C}(12)$ & $10(1)$ & $15(1)$ & $10(1)$ & $1(1)$ & $-1(1)$ & $1(1)$ \\
$\mathrm{C}(13)$ & $10(1)$ & $18(1)$ & $12(1)$ & $1(1)$ & $2(1)$ & $0(1)$ \\
$\mathrm{C}(14)$ & $12(1)$ & $17(1)$ & $12(1)$ & $-1(1)$ & $1(1)$ & $3(1)$ \\
$\mathrm{C}(15)$ & $13(1)$ & $15(1)$ & $10(1)$ & $-1(1)$ & $-1(1)$ & $1(1)$ \\
$\mathrm{C}(16)$ & $16(1)$ & $14(1)$ & $14(1)$ & $-2(1)$ & $0(1)$ & $5(1)$ \\
$\mathrm{C}(17)$ & $21(1)$ & $12(1)$ & $14(1)$ & $-1(1)$ & $0(1)$ & $2(1)$ \\
$\mathrm{C}(18)$ & $17(1)$ & $13(1)$ & $8(1)$ & 0 & $-1(1)$ & 0 \\
$\mathrm{C}(19)$ & $14(1)$ & $12(1)$ & $9(1)$ & 0 & $-1(1)$ & 0 \\
$\mathrm{C}(20)$ & $13(1)$ & $13(1)$ & $9(1)$ & $0(1)$ & $0(1)$ & $1(1)$ \\
$\mathrm{C}(21)$ & $10(1)$ & $13(1)$ & $9(1)$ & $0(1)$ & $0(1)$ & $1(1)$ \\
$\mathrm{C}(22)$ & $9(1)$ & $15(1)$ & $10(1)$ & 0 & $-1(1)$ & 0 \\
& & & & & & \\
\end{tabular}

\section{Single-crystal data for DBC}

Table 1. Crystal data and structure refinement for DBC.

Identification code

i15505

Empirical formula

C32 H16

Formula weight

400.45

Temperature

$100.0(2) \mathrm{K}$

Wavelength

Crystal system

Space group

$0.71073 \AA$

Monoclinic

Unit cell dimensions

P 21

$\mathrm{a}=5.2697(2) \AA$

$\alpha=90^{\circ}$.

$\mathrm{b}=14.6808(6) \AA$

$\beta=96.013(2)^{\circ}$.

$\mathrm{c}=11.6839(4) \AA$

$\gamma=90^{\circ}$.

Volume

Z

$898.93(6) \AA^{3}$

Density (calculated)

2

Absorption coefficient

$\mathrm{F}(000)$

Crystal size

Theta range for data collection

Index ranges

Reflections collected

Independent reflections

Completeness to theta $=25.000^{\circ}$

Absorption correction

Max. and min. transmission

Refinement method

Data / restraints / parameters

Goodness-of-fit on $\mathrm{F}^{2}$

Final $\mathrm{R}$ indices [I $>2 \operatorname{sigma}(\mathrm{I})]$

$\mathrm{R}$ indices (all data)

Absolute structure parameter

Extinction coefficient

$1.479 \mathrm{Mg} / \mathrm{m}^{3}$

$0.084 \mathrm{~mm}^{-1}$

416

$0.100 \times 0.080 \times 0.040 \mathrm{~mm}^{3}$

1.753 to $27.103^{\circ}$.

$-6<=\mathrm{h}<=6,-18<=\mathrm{k}<=18,-14<=\mathrm{l}<=14$

35392

$3962[\mathrm{R}($ int $)=0.0541]$

$100.0 \%$

Semi-empirical from equivalents

0.9705 and 0.9086

Full-matrix least-squares on $\mathrm{F}^{2}$

3962 / 1 / 290

1.044

$\mathrm{R} 1=0.0406, \mathrm{wR} 2=0.0957$

$\mathrm{R} 1=0.0565, \mathrm{wR} 2=0.1058$

0.5

$0.013(3)$

Largest diff. peak and hole

0.229 and -0.188 e. $\AA^{-3}$ 
Table 2. Atomic coordinates ( $\left.\times 10^{4}\right)$ and equivalent isotropic displacement parameters $\left(\AA^{2} \times 10^{3}\right)$ for $\mathrm{i} 15505 . \mathrm{U}(\mathrm{eq})$ is defined as one third of the trace of the orthogonalized $\mathrm{U}^{\mathrm{ij}}$ tensor.

\begin{tabular}{|c|c|c|c|c|}
\hline & $\mathrm{x}$ & $\mathrm{y}$ & $\mathrm{z}$ & $\mathrm{U}(\mathrm{eq})$ \\
\hline$C(1)$ & $3381(5)$ & 4994(2) & $6681(2)$ & $16(1)$ \\
\hline $\mathrm{C}(2)$ & $2265(5)$ & $4331(2)$ & $7406(2)$ & $16(1)$ \\
\hline C(3) & $3385(5)$ & $4104(2)$ & $8526(2)$ & $19(1)$ \\
\hline C(4) & $2245(5)$ & $3514(2)$ & $9224(2)$ & 21(1) \\
\hline$C(5)$ & $-83(5)$ & $3109(2)$ & $8832(2)$ & $22(1)$ \\
\hline$C(6)$ & $-1105(5)$ & $3248(2)$ & $7718(2)$ & $20(1)$ \\
\hline$C(7)$ & $70(5)$ & $3829(2)$ & $6965(2)$ & $15(1)$ \\
\hline $\mathrm{C}(8)$ & $-811(5)$ & $3880(2)$ & $5756(2)$ & $16(1)$ \\
\hline C(9) & $-2940(5)$ & $3367(2)$ & $5263(2)$ & $19(1)$ \\
\hline$C(10)$ & $-3636(5)$ & $3362(2)$ & $4107(2)$ & 21(1) \\
\hline $\mathrm{C}(11)$ & $-2279(5)$ & $3875(2)$ & $3349(2)$ & $20(1)$ \\
\hline$C(12)$ & $-2993(5)$ & $3883(2)$ & $2146(2)$ & $24(1)$ \\
\hline$C(13)$ & $-1692(6)$ & $4389(2)$ & $1423(3)$ & $25(1)$ \\
\hline$C(14)$ & 417(6) & 4934(2) & $1863(2)$ & $20(1)$ \\
\hline$C(15)$ & $1753(5)$ & $5486(2)$ & $1129(2)$ & $25(1)$ \\
\hline$C(16)$ & $3765(6)$ & $6006(2)$ & $1556(2)$ & $24(1)$ \\
\hline$C(17)$ & $4593(5)$ & $6022(2)$ & $2756(2)$ & $20(1)$ \\
\hline $\mathrm{C}(18)$ & $6715(5)$ & $6541(2)$ & $3218(3)$ & $22(1)$ \\
\hline$C(19)$ & $7470(5)$ & $6549(2)$ & $4370(3)$ & 21(1) \\
\hline$C(20)$ & $6119(5)$ & $6050(2)$ & $5155(2)$ & $17(1)$ \\
\hline$C(21)$ & $6749(5)$ & $6129(2)$ & $6388(2)$ & $17(1)$ \\
\hline$C(22)$ & $8735(5)$ & $6705(2)$ & $6873(3)$ & 21(1) \\
\hline $\mathrm{C}(23)$ & $9064(5)$ & $6888(2)$ & $8032(3)$ & $24(1)$ \\
\hline$C(24)$ & $7357(5)$ & $6527(2)$ & $8746(2)$ & $23(1)$ \\
\hline $\mathrm{C}(25)$ & $5485(5)$ & $5936(2)$ & $8312(2)$ & $19(1)$ \\
\hline$C(26)$ & $5232(5)$ & $5668(2)$ & $7138(2)$ & $17(1)$ \\
\hline$C(27)$ & $2661(5)$ & $4974(2)$ & $5495(2)$ & $15(1)$ \\
\hline $\mathrm{C}(28)$ & $569(5)$ & $4408(2)$ & $5022(2)$ & $15(1)$ \\
\hline$C(29)$ & $-170(5)$ & $4403(2)$ & $3813(2)$ & $17(1)$ \\
\hline$C(30)$ & $1180(5)$ & $4942(2)$ & $3058(2)$ & $18(1)$ \\
\hline$C(31)$ & $3259(5)$ & $5497(2)$ & $3517(2)$ & $17(1)$ \\
\hline$C(32)$ & $4015(5)$ & $5517(2)$ & $4729(2)$ & $16(1)$ \\
\hline
\end{tabular}

Table 3. Bond lengths $[\AA]$ and angles $\left[{ }^{\circ}\right]$ for i1 5505 .

\begin{tabular}{ll}
\hline $\mathrm{C}(1)-\mathrm{C}(27)$ & $1.398(4)$ \\
$\mathrm{C}(1)-\mathrm{C}(26)$ & $1.452(4)$ \\
$\mathrm{C}(1)-\mathrm{C}(2)$ & $1.455(4)$ \\
$\mathrm{C}(2)-\mathrm{C}(3)$ & $1.417(4)$ \\
$\mathrm{C}(2)-\mathrm{C}(7)$ & $1.421(4)$ \\
$\mathrm{C}(3)-\mathrm{C}(4)$ & $1.371(4)$ \\
$\mathrm{C}(3)-\mathrm{H}(3)$ & 0.9500 \\
$\mathrm{C}(4)-\mathrm{C}(5)$ & $1.396(4)$ \\
$\mathrm{C}(4)-\mathrm{H}(4)$ & 0.9500 \\
$\mathrm{C}(5)-\mathrm{C}(6)$ & $1.371(4)$ \\
$\mathrm{C}(5)-\mathrm{H}(5)$ & 0.9500 \\
$\mathrm{C}(6)-\mathrm{C}(7)$ & $1.414(4)$ \\
$\mathrm{C}(6)-\mathrm{H}(6)$ & 0.9500 \\
$\mathrm{C}(7)-\mathrm{C}(8)$ & $1.442(4)$ \\
$\mathrm{C}(8)-\mathrm{C}(28)$ & $1.413(4)$ \\
$\mathrm{C}(8)-\mathrm{C}(9)$ & $1.422(4)$ \\
$\mathrm{C}(9)-\mathrm{C}(10)$ & $1.362(4)$ \\
$\mathrm{C}(9)-\mathrm{H}(9)$ & 0.9500 \\
$\mathrm{C}(10)-\mathrm{C}(11)$ & $1.413(4)$ \\
$\mathrm{C}(10)-\mathrm{H}(10)$ & 0.9500 \\
$\mathrm{C}(11)-\mathrm{C}(29)$ & $1.415(4)$
\end{tabular}




\begin{tabular}{|c|c|}
\hline C(11)-C(12) & $1.416(4)$ \\
\hline$C(12)-C(13)$ & $1.363(4)$ \\
\hline $\mathrm{C}(12)-\mathrm{H}(12)$ & 0.9500 \\
\hline$C(13)-C(14)$ & $1.421(4)$ \\
\hline C(13)-H(13) & 0.9500 \\
\hline$C(14)-C(30)$ & $1.412(4)$ \\
\hline$C(14)-C(15)$ & $1.419(4)$ \\
\hline$C(15)-C(16)$ & $1.358(4)$ \\
\hline $\mathrm{C}(15)-\mathrm{H}(15)$ & 0.9500 \\
\hline C(16)-C(17) & $1.424(4)$ \\
\hline C(16)-H(16) & 0.9500 \\
\hline $\mathrm{C}(17)-\mathrm{C}(18)$ & $1.412(4)$ \\
\hline$C(17)-C(31)$ & $1.418(4)$ \\
\hline C(18)-C(19) & $1.363(4)$ \\
\hline C(18)-H(18) & 0.9500 \\
\hline C(19)-C(20) & $1.422(4)$ \\
\hline $\mathrm{C}(19)-\mathrm{H}(19)$ & 0.9500 \\
\hline$C(20)-C(32)$ & $1.405(4)$ \\
\hline $\mathrm{C}(20)-\mathrm{C}(21)$ & $1.448(4)$ \\
\hline $\mathrm{C}(21)-\mathrm{C}(22)$ & $1.417(4)$ \\
\hline $\mathrm{C}(21)-\mathrm{C}(26)$ & $1.418(4)$ \\
\hline $\mathrm{C}(22)-\mathrm{C}(23)$ & $1.374(4)$ \\
\hline $\mathrm{C}(22)-\mathrm{H}(22)$ & 0.9500 \\
\hline $\mathrm{C}(23)-\mathrm{C}(24)$ & $1.394(4)$ \\
\hline $\mathrm{C}(23)-\mathrm{H}(23)$ & 0.9500 \\
\hline $\mathrm{C}(24)-\mathrm{C}(25)$ & $1.370(4)$ \\
\hline $\mathrm{C}(24)-\mathrm{H}(24)$ & 0.9500 \\
\hline$C(25)-C(26)$ & $1.420(4)$ \\
\hline $\mathrm{C}(25)-\mathrm{H}(25)$ & 0.9500 \\
\hline $\mathrm{C}(27)-\mathrm{C}(32)$ & $1.441(4)$ \\
\hline $\mathrm{C}(27)-\mathrm{C}(28)$ & $1.443(3)$ \\
\hline $\mathrm{C}(28)-\mathrm{C}(29)$ & $1.426(3)$ \\
\hline $\mathrm{C}(29)-\mathrm{C}(30)$ & $1.429(4)$ \\
\hline $\mathrm{C}(30)-\mathrm{C}(31)$ & $1.424(4)$ \\
\hline $\mathrm{C}(31)-\mathrm{C}(32)$ & $1.431(3)$ \\
\hline$C(27)-C(1)-C(26)$ & $118.8(2)$ \\
\hline$C(27)-C(1)-C(2)$ & $118.6(2)$ \\
\hline $\mathrm{C}(26)-\mathrm{C}(1)-\mathrm{C}(2)$ & $122.6(2)$ \\
\hline$C(3)-C(2)-C(7)$ & $117.3(2)$ \\
\hline$C(3)-C(2)-C(1)$ & $122.7(2)$ \\
\hline $\mathrm{C}(7)-\mathrm{C}(2)-\mathrm{C}(1)$ & $119.9(2)$ \\
\hline $\mathrm{C}(4)-\mathrm{C}(3)-\mathrm{C}(2)$ & $122.1(3)$ \\
\hline $\mathrm{C}(4)-\mathrm{C}(3)-\mathrm{H}(3)$ & 119.0 \\
\hline $\mathrm{C}(2)-\mathrm{C}(3)-\mathrm{H}(3)$ & 119.0 \\
\hline$C(3)-C(4)-C(5)$ & $119.9(3)$ \\
\hline $\mathrm{C}(3)-\mathrm{C}(4)-\mathrm{H}(4)$ & 120.0 \\
\hline $\mathrm{C}(5)-\mathrm{C}(4)-\mathrm{H}(4)$ & 120.0 \\
\hline $\mathrm{C}(6)-\mathrm{C}(5)-\mathrm{C}(4)$ & $119.5(3)$ \\
\hline $\mathrm{C}(6)-\mathrm{C}(5)-\mathrm{H}(5)$ & 120.2 \\
\hline $\mathrm{C}(4)-\mathrm{C}(5)-\mathrm{H}(5)$ & 120.2 \\
\hline $\mathrm{C}(5)-\mathrm{C}(6)-\mathrm{C}(7)$ & $121.8(3)$ \\
\hline $\mathrm{C}(5)-\mathrm{C}(6)-\mathrm{H}(6)$ & 119.1 \\
\hline $\mathrm{C}(7)-\mathrm{C}(6)-\mathrm{H}(6)$ & 119.1 \\
\hline$C(6)-C(7)-C(2)$ & $118.6(2)$ \\
\hline $\mathrm{C}(6)-\mathrm{C}(7)-\mathrm{C}(8)$ & $121.6(2)$ \\
\hline $\mathrm{C}(2)-\mathrm{C}(7)-\mathrm{C}(8)$ & $119.7(2)$ \\
\hline $\mathrm{C}(28)-\mathrm{C}(8)-\mathrm{C}(9)$ & $118.7(2)$ \\
\hline $\mathrm{C}(28)-\mathrm{C}(8)-\mathrm{C}(7)$ & 119.3(2) \\
\hline$C(9)-C(8)-C(7)$ & $121.9(2)$ \\
\hline $\mathrm{C}(10)-\mathrm{C}(9)-\mathrm{C}(8)$ & $121.5(3)$ \\
\hline $\mathrm{C}(10)-\mathrm{C}(9)-\mathrm{H}(9)$ & 119.2 \\
\hline
\end{tabular}




\begin{tabular}{|c|c|}
\hline $\mathrm{C}(8)-\mathrm{C}(9)-\mathrm{H}(9)$ & 119.2 \\
\hline$C(9)-C(10)-C(11)$ & $121.2(3)$ \\
\hline $\mathrm{C}(9)-\mathrm{C}(10)-\mathrm{H}(10)$ & 119.4 \\
\hline $\mathrm{C}(11)-\mathrm{C}(10)-\mathrm{H}(10)$ & 119.4 \\
\hline$C(10)-C(11)-C(29)$ & $118.7(2)$ \\
\hline$C(10)-C(11)-C(12)$ & $121.8(3)$ \\
\hline$C(29)-C(11)-C(12)$ & $119.5(3)$ \\
\hline$C(13)-C(12)-C(11)$ & $121.4(3)$ \\
\hline $\mathrm{C}(13)-\mathrm{C}(12)-\mathrm{H}(12)$ & 119.3 \\
\hline $\mathrm{C}(11)-\mathrm{C}(12)-\mathrm{H}(12)$ & 119.3 \\
\hline $\mathrm{C}(12)-\mathrm{C}(13)-\mathrm{C}(14)$ & $120.5(3)$ \\
\hline $\mathrm{C}(12)-\mathrm{C}(13)-\mathrm{H}(13)$ & 119.7 \\
\hline $\mathrm{C}(14)-\mathrm{C}(13)-\mathrm{H}(13)$ & 119.7 \\
\hline$C(30)-C(14)-C(15)$ & $119.0(3)$ \\
\hline$C(30)-C(14)-C(13)$ & $119.5(3)$ \\
\hline $\mathrm{C}(15)-\mathrm{C}(14)-\mathrm{C}(13)$ & $121.5(3)$ \\
\hline $\mathrm{C}(16)-\mathrm{C}(15)-\mathrm{C}(14)$ & $121.1(3)$ \\
\hline $\mathrm{C}(16)-\mathrm{C}(15)-\mathrm{H}(15)$ & 119.5 \\
\hline $\mathrm{C}(14)-\mathrm{C}(15)-\mathrm{H}(15)$ & 119.5 \\
\hline$C(15)-C(16)-C(17)$ & $121.2(3)$ \\
\hline $\mathrm{C}(15)-\mathrm{C}(16)-\mathrm{H}(16)$ & 119.4 \\
\hline $\mathrm{C}(17)-\mathrm{C}(16)-\mathrm{H}(16)$ & 119.4 \\
\hline $\mathrm{C}(18)-\mathrm{C}(17)-\mathrm{C}(31)$ & $118.6(3)$ \\
\hline $\mathrm{C}(18)-\mathrm{C}(17)-\mathrm{C}(16)$ & $122.2(3)$ \\
\hline$C(31)-C(17)-C(16)$ & 119.1(3) \\
\hline $\mathrm{C}(19)-\mathrm{C}(18)-\mathrm{C}(17)$ & $121.3(3)$ \\
\hline $\mathrm{C}(19)-\mathrm{C}(18)-\mathrm{H}(18)$ & 119.4 \\
\hline $\mathrm{C}(17)-\mathrm{C}(18)-\mathrm{H}(18)$ & 119.4 \\
\hline $\mathrm{C}(18)-\mathrm{C}(19)-\mathrm{C}(20)$ & $121.2(3)$ \\
\hline $\mathrm{C}(18)-\mathrm{C}(19)-\mathrm{H}(19)$ & 119.4 \\
\hline $\mathrm{C}(20)-\mathrm{C}(19)-\mathrm{H}(19)$ & 119.4 \\
\hline$C(32)-C(20)-C(19)$ & $119.3(2)$ \\
\hline$C(32)-C(20)-C(21)$ & $119.1(2)$ \\
\hline$C(19)-C(20)-C(21)$ & $121.5(2)$ \\
\hline $\mathrm{C}(22)-\mathrm{C}(21)-\mathrm{C}(26)$ & $118.5(3)$ \\
\hline $\mathrm{C}(22)-\mathrm{C}(21)-\mathrm{C}(20)$ & $121.8(2)$ \\
\hline$C(26)-C(21)-C(20)$ & $119.5(2)$ \\
\hline$C(23)-C(22)-C(21)$ & $121.5(3)$ \\
\hline $\mathrm{C}(23)-\mathrm{C}(22)-\mathrm{H}(22)$ & 119.2 \\
\hline $\mathrm{C}(21)-\mathrm{C}(22)-\mathrm{H}(22)$ & 119.2 \\
\hline $\mathrm{C}(22)-\mathrm{C}(23)-\mathrm{C}(24)$ & $119.5(3)$ \\
\hline $\mathrm{C}(22)-\mathrm{C}(23)-\mathrm{H}(23)$ & 120.2 \\
\hline $\mathrm{C}(24)-\mathrm{C}(23)-\mathrm{H}(23)$ & 120.2 \\
\hline $\mathrm{C}(25)-\mathrm{C}(24)-\mathrm{C}(23)$ & $120.2(3)$ \\
\hline $\mathrm{C}(25)-\mathrm{C}(24)-\mathrm{H}(24)$ & 119.9 \\
\hline $\mathrm{C}(23)-\mathrm{C}(24)-\mathrm{H}(24)$ & 119.9 \\
\hline$C(24)-C(25)-C(26)$ & $121.6(3)$ \\
\hline $\mathrm{C}(24)-\mathrm{C}(25)-\mathrm{H}(25)$ & 119.2 \\
\hline $\mathrm{C}(26)-\mathrm{C}(25)-\mathrm{H}(25)$ & 119.2 \\
\hline$C(21)-C(26)-C(25)$ & $117.6(2)$ \\
\hline $\mathrm{C}(21)-\mathrm{C}(26)-\mathrm{C}(1)$ & $119.8(2)$ \\
\hline$C(25)-C(26)-C(1)$ & $122.4(2)$ \\
\hline $\mathrm{C}(1)-\mathrm{C}(27)-\mathrm{C}(32)$ & $120.2(2)$ \\
\hline $\mathrm{C}(1)-\mathrm{C}(27)-\mathrm{C}(28)$ & $120.6(2)$ \\
\hline $\mathrm{C}(32)-\mathrm{C}(27)-\mathrm{C}(28)$ & $119.2(2)$ \\
\hline $\mathrm{C}(8)-\mathrm{C}(28)-\mathrm{C}(29)$ & $119.6(2)$ \\
\hline $\mathrm{C}(8)-\mathrm{C}(28)-\mathrm{C}(27)$ & $120.3(2)$ \\
\hline $\mathrm{C}(29)-\mathrm{C}(28)-\mathrm{C}(27)$ & $120.1(2)$ \\
\hline $\mathrm{C}(11)-\mathrm{C}(29)-\mathrm{C}(28)$ & $120.3(2)$ \\
\hline $\mathrm{C}(11)-\mathrm{C}(29)-\mathrm{C}(30)$ & $119.3(2)$ \\
\hline $\mathrm{C}(28)-\mathrm{C}(29)-\mathrm{C}(30)$ & $120.4(2)$ \\
\hline $\mathrm{C}(14)-\mathrm{C}(30)-\mathrm{C}(31)$ & $120.3(2)$ \\
\hline
\end{tabular}




$\begin{array}{ll}\mathrm{C}(14)-\mathrm{C}(30)-\mathrm{C}(29) & 119.8(2) \\ \mathrm{C}(31)-\mathrm{C}(30)-\mathrm{C}(29) & 119.8(2) \\ \mathrm{C}(17)-\mathrm{C}(31)-\mathrm{C}(30) & 119.2(2) \\ \mathrm{C}(17)-\mathrm{C}(31)-\mathrm{C}(32) & 120.3(2) \\ \mathrm{C}(30)-\mathrm{C}(31)-\mathrm{C}(32) & 120.5(2) \\ \mathrm{C}(20)-\mathrm{C}(32)-\mathrm{C}(31) & 119.3(2) \\ \mathrm{C}(20)-\mathrm{C}(32)-\mathrm{C}(27) & 120.8(2) \\ \mathrm{C}(31)-\mathrm{C}(32)-\mathrm{C}(27) & 119.9(2)\end{array}$

Symmetry transformations used to generate equivalent atoms:

Table 4. Anisotropic displacement parameters $\left(\AA^{2} \times 10^{3}\right)$ for i15505. The anisotropic displacement factor exponent takes the form: $-2 \pi^{2}\left[\mathrm{~h}^{2} \mathrm{a} * 2 \mathrm{U}^{11}+\ldots+2 \mathrm{~h} \mathrm{k} \mathrm{a}^{*} \mathrm{~b}^{*} \mathrm{U}^{12}\right]$

\begin{tabular}{|c|c|c|c|c|c|c|}
\hline & $\mathrm{U}^{11}$ & $\mathrm{U}^{22}$ & $\mathrm{U}^{33}$ & $\mathrm{U}^{23}$ & $\mathrm{U}^{13}$ & $\mathrm{U}^{12}$ \\
\hline $\mathrm{C}(1)$ & $14(1)$ & $15(1)$ & $18(1)$ & $-1(1)$ & $0(1)$ & $2(1)$ \\
\hline$C(2)$ & $15(1)$ & $15(1)$ & $17(1)$ & $0(1)$ & $3(1)$ & $2(1)$ \\
\hline$C(3)$ & 21(1) & $19(1)$ & $18(1)$ & $-4(1)$ & 1(1) & $1(1)$ \\
\hline C(4) & $26(1)$ & $19(2)$ & $18(1)$ & $0(1)$ & $2(1)$ & $2(1)$ \\
\hline$C(5)$ & $27(2)$ & $18(2)$ & $23(2)$ & $3(1)$ & $7(1)$ & $0(1)$ \\
\hline$C(6)$ & $17(1)$ & $17(1)$ & $25(2)$ & $0(1)$ & $4(1)$ & $-1(1)$ \\
\hline C(7) & $16(1)$ & $11(1)$ & $19(1)$ & $-2(1)$ & $3(1)$ & $2(1)$ \\
\hline $\mathrm{C}(8)$ & $14(1)$ & $13(1)$ & $20(1)$ & $0(1)$ & $2(1)$ & $3(1)$ \\
\hline C(9) & $17(1)$ & $15(1)$ & $26(2)$ & $-1(1)$ & $0(1)$ & $1(1)$ \\
\hline$C(10)$ & $17(1)$ & $14(1)$ & $30(2)$ & $-5(1)$ & $-2(1)$ & $0(1)$ \\
\hline $\mathrm{C}(11)$ & $20(1)$ & $18(1)$ & $21(1)$ & $-5(1)$ & $-3(1)$ & $5(1)$ \\
\hline$C(12)$ & 21(1) & $24(2)$ & $24(2)$ & $-7(1)$ & $-6(1)$ & $8(1)$ \\
\hline $\mathrm{C}(13)$ & $28(2)$ & $29(2)$ & $16(2)$ & $-5(1)$ & $-4(1)$ & $12(1)$ \\
\hline$C(14)$ & $24(1)$ & $20(2)$ & $17(2)$ & $-2(1)$ & $2(1)$ & $12(1)$ \\
\hline$C(15)$ & $31(2)$ & $29(2)$ & $17(2)$ & $2(1)$ & $4(1)$ & $15(1)$ \\
\hline$C(16)$ & $28(2)$ & $24(2)$ & $21(2)$ & $4(1)$ & $9(1)$ & 11(1) \\
\hline$C(17)$ & $22(1)$ & $19(2)$ & $20(2)$ & $3(1)$ & $7(1)$ & $7(1)$ \\
\hline $\mathrm{C}(18)$ & $22(1)$ & $18(1)$ & $27(2)$ & $6(1)$ & 11(1) & $4(1)$ \\
\hline$C(19)$ & $17(1)$ & $15(1)$ & $31(2)$ & 1(1) & $5(1)$ & $0(1)$ \\
\hline $\mathrm{C}(20)$ & $16(1)$ & $12(1)$ & $23(2)$ & 1(1) & $4(1)$ & $4(1)$ \\
\hline $\mathrm{C}(21)$ & $14(1)$ & $12(1)$ & $25(2)$ & $1(1)$ & $0(1)$ & $2(1)$ \\
\hline $\mathrm{C}(22)$ & $16(1)$ & $16(1)$ & $30(2)$ & $0(1)$ & $3(1)$ & $-1(1)$ \\
\hline $\mathrm{C}(23)$ & $19(1)$ & $20(2)$ & $31(2)$ & $-3(1)$ & $-3(1)$ & $-5(1)$ \\
\hline$C(24)$ & $25(1)$ & $22(2)$ & $22(2)$ & $-5(1)$ & $-1(1)$ & $-1(1)$ \\
\hline $\mathrm{C}(25)$ & $19(1)$ & $18(1)$ & $20(2)$ & 1(1) & 1(1) & $-2(1)$ \\
\hline$C(26)$ & $14(1)$ & $17(1)$ & $18(2)$ & $-1(1)$ & $0(1)$ & $2(1)$ \\
\hline $\mathrm{C}(27)$ & $13(1)$ & $12(1)$ & $19(1)$ & $-1(1)$ & 1(1) & $3(1)$ \\
\hline $\mathrm{C}(28)$ & $14(1)$ & $12(1)$ & 19(1) & $-2(1)$ & $0(1)$ & $3(1)$ \\
\hline$C(29)$ & $16(1)$ & $15(1)$ & $20(2)$ & $-2(1)$ & $2(1)$ & $6(1)$ \\
\hline $\mathrm{C}(30)$ & $19(1)$ & $16(1)$ & $17(1)$ & $-2(1)$ & $2(1)$ & $7(1)$ \\
\hline $\mathrm{C}(31)$ & $19(1)$ & $15(1)$ & $19(2)$ & $2(1)$ & $5(1)$ & $7(1)$ \\
\hline$C(32)$ & $16(1)$ & $15(1)$ & $16(2)$ & $0(1)$ & $4(1)$ & $4(1)$ \\
\hline
\end{tabular}

\section{Single-crystal data for Flu-DBC:}

Table 1. Crystal data and structure refinement for Flu-DBC.

Identification code

Empirical formula

ch17382

Formula weight

C32 H14 F2

Temperature

Wavelength

436.43

200(2) K

Crystal system

Space group

$0.71073 \AA$

Orthorhombic

P 212121 


$\begin{array}{lll}\text { Unit cell dimensions } & \mathrm{a}=3.8133(15) \AA & \alpha=90^{\circ} . \\ & \mathrm{b}=20.154(7) \AA & \beta=90^{\circ} . \\ & \mathrm{c}=23.688(9) \AA & \gamma=90^{\circ} . \\ \text { Volume } & 1820.5(12) \AA^{3} & \\ \text { Z } & 4 \\ \text { Density (calculated) } & 1.592 \mathrm{Mg} / \mathrm{m}^{3} & \\ \text { Absorption coefficient } & 0.105 \mathrm{~mm}^{-1} & \\ \mathrm{~F}(000) & 896 \\ \text { Crystal size } & 0.79 \times 0.04 \times 0.01 \mathrm{~mm}^{3} \\ \text { Theta range for data collection } & 1.33 \text { to } 25.10^{\circ} . \\ \text { Index ranges } & -4<=\mathrm{h}<=3,-24<=\mathrm{k}<=23,-28<=1<=27 \\ \text { Reflections collected } & 9342 \\ \text { Independent reflections } & 3238[\mathrm{R}(\mathrm{int})=0.0772] \\ \text { Completeness to theta }=25.10^{\circ} & 99.6 \% \\ \text { Absorption correction } & \mathrm{Semi}-\mathrm{empirical} \text { from equivalents } \\ \text { Max. and min. transmission } & 0.9989 \text { and } 0.9214 & \\ \text { Refinement method } & \mathrm{Full}-\mathrm{matrix} \text { least-squares on } \mathrm{F}^{2} \\ \text { Data / restraints / parameters } & 3238 / 0 / 307 \\ \text { Goodness-of-fit on } \mathrm{F}^{2} & 1.057 \\ \text { Final R indices [I } 2 \text { sigma(I)] } & \mathrm{R} 1=0.0737, \mathrm{wR} 2=0.1840 \\ \mathrm{R} \text { indices (all data) } & \mathrm{R} 1=0.1202, \mathrm{wR} 2=0.2278 \\ \text { Absolute structure parameter } & -1(2) \\ \text { Largest diff. peak and hole } & 0.584 \text { and }-0.603 \text { e. } \AA^{-3}\end{array}$

Table 2. Atomic coordinates ( $\left.\times 10^{4}\right)$ and equivalent isotropic displacement parameters $\left(\AA^{2} \times 10^{3}\right)$ for flu-dbc. $U(e q)$ is defined as one third of the trace of the orthogonalized $U^{i j}$ tensor.

\begin{tabular}{lrrrr}
\hline & $\mathrm{x}$ & $\mathrm{y}$ & $\mathrm{z}$ & $\mathrm{U}(\mathrm{eq})$ \\
\hline $\mathrm{C}(1)$ & $11164(16)$ & $10230(2)$ & $9047(3)$ & $31(2)$ \\
$\mathrm{C}(2)$ & $11614(15)$ & $10166(2)$ & $8481(2)$ & $26(1)$ \\
$\mathrm{C}(3)$ & $10374(13)$ & $9586(2)$ & $8204(2)$ & $19(1)$ \\
$\mathrm{C}(4)$ & $9063(14)$ & $9059(2)$ & $8538(2)$ & $19(1)$ \\
$\mathrm{C}(5)$ & $8428(15)$ & $9181(2)$ & $9117(2)$ & $26(1)$ \\
$\mathrm{C}(6)$ & $9537(15)$ & $9756(2)$ & $9372(3)$ & $28(1)$ \\
$\mathrm{C}(7)$ & $10237(14)$ & $9544(2)$ & $7599(2)$ & $19(1)$ \\
$\mathrm{C}(8)$ & $11442(15)$ & $10073(2)$ & $7254(2)$ & $25(1)$ \\
$\mathrm{C}(9)$ & $11054(15)$ & $10056(2)$ & $6684(2)$ & $27(1)$ \\
$\mathrm{C}(10)$ & $9460(14)$ & $9509(2)$ & $6407(2)$ & $21(1)$ \\
$\mathrm{C}(11)$ & $8993(15)$ & $9489(3)$ & $5813(2)$ & $28(1)$ \\
$\mathrm{C}(12)$ & $7517(15)$ & $8961(2)$ & $5561(2)$ & $26(1)$ \\
$\mathrm{C}(13)$ & $6361(14)$ & $8407(2)$ & $5874(2)$ & $21(1)$ \\
$\mathrm{C}(14)$ & $6789(13)$ & $8413(2)$ & $6466(2)$ & $18(1)$ \\
$\mathrm{C}(15)$ & $8347(14)$ & $8968(2)$ & $6739(2)$ & $20(1)$ \\
$\mathrm{C}(16)$ & $8743(13)$ & $8989(2)$ & $7338(2)$ & $17(1)$ \\
$\mathrm{C}(17)$ & $7680(14)$ & $8419(2)$ & $7674(2)$ & $18(1)$ \\
$\mathrm{C}(18)$ & $6148(13)$ & $7850(2)$ & $7394(2)$ & $18(1)$ \\
$\mathrm{C}(19)$ & $5706(14)$ & $7858(2)$ & $6793(2)$ & $17(1)$ \\
$\mathrm{C}(20)$ & $4151(14)$ & $7308(2)$ & $6517(2)$ & $22(1)$ \\
$\mathrm{C}(21)$ & $3736(15)$ & $7314(2)$ & $5922(2)$ & $26(1)$ \\
$\mathrm{C}(22)$ & $4804(14)$ & $7850(3)$ & $5617(2)$ & $27(1)$ \\
$\mathrm{C}(23)$ & $3018(14)$ & $6762(2)$ & $6844(2)$ & $25(1)$ \\
$\mathrm{C}(24)$ & $3431(15)$ & $6760(2)$ & $7417(2)$ & $26(1)$ \\
$\mathrm{C}(25)$ & $5132(14)$ & $7295(2)$ & $7707(2)$ & $20(1)$ \\
$\mathrm{C}(26)$ & $5902(15)$ & $7268(2)$ & $8303(2)$ & $23(1)$ \\
$\mathrm{C}(27)$ & $5231(14)$ & $6688(2)$ & $8620(2)$ & $24(1)$ \\
& & & &
\end{tabular}


Supporting Information

\begin{tabular}{lrrrr}
$\mathrm{C}(28)$ & $6365(17)$ & $6652(2)$ & $9159(2)$ & $28(1)$ \\
$\mathrm{C}(29)$ & $8320(15)$ & $7142(2)$ & $9422(2)$ & $23(1)$ \\
$\mathrm{C}(30)$ & $8874(15)$ & $7722(2)$ & $9122(2)$ & $24(1)$ \\
$\mathrm{C}(31)$ & $7526(14)$ & $7821(2)$ & $8570(2)$ & $20(1)$ \\
$\mathrm{C}(32)$ & $8071(15)$ & $8433(2)$ & $8262(2)$ & $22(1)$ \\
$\mathrm{F}(1)$ & $12371(10)$ & $10792(1)$ & $9305(1)$ & $41(1)$ \\
$\mathrm{F}(2)$ & $5653(11)$ & $6087(1)$ & $9467(1)$ & $42(1)$ \\
\hline
\end{tabular}

Table 3. Bond lengths $[\AA]$ and angles $\left[{ }^{\circ}\right]$ for flu-dbc.

\begin{tabular}{ll}
\hline $\mathrm{C}(1)-\mathrm{C}(2)$ & $1.358(7)$ \\
$\mathrm{C}(1)-\mathrm{F}(1)$ & $1.367(6)$ \\
$\mathrm{C}(1)-\mathrm{C}(6)$ & $1.375(7)$ \\
$\mathrm{C}(2)-\mathrm{C}(3)$ & $1.422(7)$ \\
$\mathrm{C}(2)-\mathrm{H}(2)$ & 0.9500 \\
$\mathrm{C}(3)-\mathrm{C}(4)$ & $1.415(7)$ \\
$\mathrm{C}(3)-\mathrm{C}(7)$ & $1.437(7)$ \\
$\mathrm{C}(4)-\mathrm{C}(5)$ & $1.414(7)$ \\
$\mathrm{C}(4)-\mathrm{C}(32)$ & $1.470(6)$ \\
$\mathrm{C}(5)-\mathrm{C}(6)$ & $1.373(7)$ \\
$\mathrm{C}(5)-\mathrm{H}(5)$ & 0.9500 \\
$\mathrm{C}(6)-\mathrm{H}(6)$ & 0.9500 \\
$\mathrm{C}(7)-\mathrm{C}(16)$ & $1.399(7)$ \\
$\mathrm{C}(7)-\mathrm{C}(8)$ & $1.420(7)$ \\
$\mathrm{C}(8)-\mathrm{C}(9)$ & $1.358(7)$ \\
$\mathrm{C}(8)-\mathrm{H}(8)$ & 0.9500 \\
$\mathrm{C}(9)-\mathrm{C}(10)$ & $1.420(7)$ \\
$\mathrm{C}(9)-\mathrm{H}(9)$ & 0.9500 \\
$\mathrm{C}(10)-\mathrm{C}(15)$ & $1.410(7)$ \\
$\mathrm{C}(10)-\mathrm{C}(11)$ & $1.419(7)$ \\
$\mathrm{C}(11)-\mathrm{C}(12)$ & $1.345(7)$ \\
$\mathrm{C}(11)-\mathrm{H}(11)$ & 0.9500 \\
$\mathrm{C}(12)-\mathrm{C}(13)$ & $1.410(7)$ \\
$\mathrm{C}(12)-\mathrm{H}(12)$ & 0.9500 \\
$\mathrm{C}(13)-\mathrm{C}(22)$ & $1.409(7)$ \\
$\mathrm{C}(13)-\mathrm{C}(14)$ & $1.412(7)$ \\
$\mathrm{C}(14)-\mathrm{C}(19)$ & $1.422(7)$ \\
$\mathrm{C}(14)-\mathrm{C}(15)$ & $1.423(7)$ \\
$\mathrm{C}(15)-\mathrm{C}(16)$ & $1.428(7)$ \\
$\mathrm{C}(16)-\mathrm{C}(17)$ & $1.455(7)$ \\
$\mathrm{C}(17)-\mathrm{C}(32)$ & $1.402(7)$ \\
$\mathrm{C}(17)-\mathrm{C}(18)$ & $1.447(6)$ \\
$\mathrm{C}(18)-\mathrm{C}(25)$ & $1.396(7)$ \\
$\mathrm{C}(18)-\mathrm{C}(19)$ & $1.435(7)$ \\
$\mathrm{C}(19)-\mathrm{C}(20)$ & $1.415(7)$ \\
$\mathrm{C}(20)-\mathrm{C}(23)$ & $1.412(7)$ \\
$\mathrm{C}(20)-\mathrm{C}(21)$ & $1.419(7)$ \\
$\mathrm{C}(21)-\mathrm{C}(22)$ & $1.363(7)$ \\
$\mathrm{C}(21)-\mathrm{H}(21)$ & 0.9500 \\
$\mathrm{C}(22)-\mathrm{H}(22)$ & 0.9500 \\
$\mathrm{C}(23)-\mathrm{C}(24)$ & $1.367(7)$ \\
$\mathrm{C}(23)-\mathrm{H}(23)$ & 0.9500 \\
$\mathrm{C}(24)-\mathrm{C}(25)$ & $1.435(7)$ \\
$\mathrm{C}(24)-\mathrm{H}(24)$ & 0.9500 \\
&
\end{tabular}




\begin{tabular}{|c|c|}
\hline$C(25)-C(26)$ & $1.443(7)$ \\
\hline$C(26)-C(27)$ & $1.412(7)$ \\
\hline$C(26)-C(31)$ & $1.423(7)$ \\
\hline$C(27)-C(28)$ & $1.349(7)$ \\
\hline $\mathrm{C}(27)-\mathrm{H}(27)$ & 0.9500 \\
\hline$C(28)-F(2)$ & $1.381(5)$ \\
\hline $\mathrm{C}(28)-\mathrm{C}(29)$ & $1.386(7)$ \\
\hline $\mathrm{C}(29)-\mathrm{C}(30)$ & $1.385(7)$ \\
\hline $\mathrm{C}(29)-\mathrm{H}(29)$ & 0.9500 \\
\hline $\mathrm{C}(30)-\mathrm{C}(31)$ & $1.419(7)$ \\
\hline C(30)-H(30) & 0.9500 \\
\hline $\mathrm{C}(31)-\mathrm{C}(32)$ & $1.448(6)$ \\
\hline $\mathrm{C}(2)-\mathrm{C}(1)-\mathrm{F}(1)$ & $118.5(5)$ \\
\hline$C(2)-C(1)-C(6)$ & $123.0(5)$ \\
\hline $\mathrm{F}(1)-\mathrm{C}(1)-\mathrm{C}(6)$ & $118.5(5)$ \\
\hline $\mathrm{C}(1)-\mathrm{C}(2)-\mathrm{C}(3)$ & $119.4(5)$ \\
\hline $\mathrm{C}(1)-\mathrm{C}(2)-\mathrm{H}(2)$ & 120.3 \\
\hline $\mathrm{C}(3)-\mathrm{C}(2)-\mathrm{H}(2)$ & 120.3 \\
\hline $\mathrm{C}(4)-\mathrm{C}(3)-\mathrm{C}(2)$ & $118.4(5)$ \\
\hline$C(4)-C(3)-C(7)$ & $120.0(5)$ \\
\hline$C(2)-C(3)-C(7)$ & $121.4(5)$ \\
\hline $\mathrm{C}(5)-\mathrm{C}(4)-\mathrm{C}(3)$ & $118.2(5)$ \\
\hline $\mathrm{C}(5)-\mathrm{C}(4)-\mathrm{C}(32)$ & $122.4(5)$ \\
\hline $\mathrm{C}(3)-\mathrm{C}(4)-\mathrm{C}(32)$ & $119.1(5)$ \\
\hline $\mathrm{C}(6)-\mathrm{C}(5)-\mathrm{C}(4)$ & $121.4(5)$ \\
\hline $\mathrm{C}(6)-\mathrm{C}(5)-\mathrm{H}(5)$ & 119.3 \\
\hline $\mathrm{C}(4)-\mathrm{C}(5)-\mathrm{H}(5)$ & 119.3 \\
\hline$C(5)-C(6)-C(1)$ & $118.5(5)$ \\
\hline $\mathrm{C}(5)-\mathrm{C}(6)-\mathrm{H}(6)$ & 120.8 \\
\hline $\mathrm{C}(1)-\mathrm{C}(6)-\mathrm{H}(6)$ & 120.8 \\
\hline $\mathrm{C}(16)-\mathrm{C}(7)-\mathrm{C}(8)$ & $118.6(5)$ \\
\hline$C(16)-C(7)-C(3)$ & $120.1(5)$ \\
\hline C(8)-C(7)-C(3) & $121.2(5)$ \\
\hline $\mathrm{C}(9)-\mathrm{C}(8)-\mathrm{C}(7)$ & $121.1(5)$ \\
\hline $\mathrm{C}(9)-\mathrm{C}(8)-\mathrm{H}(8)$ & 119.4 \\
\hline $\mathrm{C}(7)-\mathrm{C}(8)-\mathrm{H}(8)$ & 119.4 \\
\hline $\mathrm{C}(8)-\mathrm{C}(9)-\mathrm{C}(10)$ & $121.8(5)$ \\
\hline $\mathrm{C}(8)-\mathrm{C}(9)-\mathrm{H}(9)$ & 119.1 \\
\hline $\mathrm{C}(10)-\mathrm{C}(9)-\mathrm{H}(9)$ & 119.1 \\
\hline$C(15)-C(10)-C(11)$ & $119.6(5)$ \\
\hline$C(15)-C(10)-C(9)$ & $118.1(5)$ \\
\hline $\mathrm{C}(11)-\mathrm{C}(10)-\mathrm{C}(9)$ & $122.3(5)$ \\
\hline $\mathrm{C}(12)-\mathrm{C}(11)-\mathrm{C}(10)$ & $121.0(5)$ \\
\hline $\mathrm{C}(12)-\mathrm{C}(11)-\mathrm{H}(11)$ & 119.5 \\
\hline $\mathrm{C}(10)-\mathrm{C}(11)-\mathrm{H}(11)$ & 119.5 \\
\hline $\mathrm{C}(11)-\mathrm{C}(12)-\mathrm{C}(13)$ & $121.6(5)$ \\
\hline $\mathrm{C}(11)-\mathrm{C}(12)-\mathrm{H}(12)$ & 119.2 \\
\hline $\mathrm{C}(13)-\mathrm{C}(12)-\mathrm{H}(12)$ & 119.2 \\
\hline $\mathrm{C}(22)-\mathrm{C}(13)-\mathrm{C}(12)$ & $122.3(5)$ \\
\hline $\mathrm{C}(22)-\mathrm{C}(13)-\mathrm{C}(14)$ & $119.0(5)$ \\
\hline $\mathrm{C}(12)-\mathrm{C}(13)-\mathrm{C}(14)$ & $118.7(5)$ \\
\hline $\mathrm{C}(13)-\mathrm{C}(14)-\mathrm{C}(19)$ & $120.1(4)$ \\
\hline $\mathrm{C}(13)-\mathrm{C}(14)-\mathrm{C}(15)$ & $120.4(4)$ \\
\hline$C(19)-C(14)-C(15)$ & $119.5(5)$ \\
\hline $\mathrm{C}(10)-\mathrm{C}(15)-\mathrm{C}(14)$ & $118.7(5)$ \\
\hline
\end{tabular}




$\begin{array}{ll}\mathrm{C}(10)-\mathrm{C}(15)-\mathrm{C}(16) & 120.0(5) \\ \mathrm{C}(14)-\mathrm{C}(15)-\mathrm{C}(16) & 121.3(4) \\ \mathrm{C}(7)-\mathrm{C}(16)-\mathrm{C}(15) & 120.3(4) \\ \mathrm{C}(7)-\mathrm{C}(16)-\mathrm{C}(17) & 120.3(5) \\ \mathrm{C}(15)-\mathrm{C}(16)-\mathrm{C}(17) & 119.4(4) \\ \mathrm{C}(32)-\mathrm{C}(17)-\mathrm{C}(18) & 120.9(4) \\ \mathrm{C}(32)-\mathrm{C}(17)-\mathrm{C}(16) & 119.8(4) \\ \mathrm{C}(18)-\mathrm{C}(17)-\mathrm{C}(16) & 119.2(5) \\ \mathrm{C}(25)-\mathrm{C}(18)-\mathrm{C}(19) & 120.2(5) \\ \mathrm{C}(25)-\mathrm{C}(18)-\mathrm{C}(17) & 120.2(5) \\ \mathrm{C}(19)-\mathrm{C}(18)-\mathrm{C}(17) & 119.6(4) \\ \mathrm{C}(20)-\mathrm{C}(19)-\mathrm{C}(14) & 119.0(5) \\ \mathrm{C}(20)-\mathrm{C}(19)-\mathrm{C}(18) & 119.9(4) \\ \mathrm{C}(14)-\mathrm{C}(19)-\mathrm{C}(18) & 121.0(4) \\ \mathrm{C}(23)-\mathrm{C}(20)-\mathrm{C}(19) & 119.0(5) \\ \mathrm{C}(23)-\mathrm{C}(20)-\mathrm{C}(21) & 121.0(5) \\ \mathrm{C}(19)-\mathrm{C}(20)-\mathrm{C}(21) & 119.9(5) \\ \mathrm{C}(22)-\mathrm{C}(21)-\mathrm{C}(20) & 120.0(5) \\ \mathrm{C}(22)-\mathrm{C}(21)-\mathrm{H}(21) & 120.0 \\ \mathrm{C}(20)-\mathrm{C}(21)-\mathrm{H}(21) & 120.0 \\ \mathrm{C}(21)-\mathrm{C}(22)-\mathrm{C}(13) & 121.9(5) \\ \mathrm{C}(21)-\mathrm{C}(22)-\mathrm{H}(22) & 119.0 \\ \mathrm{C}(13)-\mathrm{C}(22)-\mathrm{H}(22) & 119.0 \\ \mathrm{C}(24)-\mathrm{C}(23)-\mathrm{C}(20) & 120.7(5) \\ \mathrm{C}(24)-\mathrm{C}(23)-\mathrm{H}(23) & 119.6 \\ \mathrm{C}(20)-\mathrm{C}(23)-\mathrm{H}(23) & 119.6 \\ \mathrm{C}(23)-\mathrm{C}(24)-\mathrm{C}(25) & 121.7(5) \\ \mathrm{C}(23)-\mathrm{C}(24)-\mathrm{H}(24) & 119.2 \\ \mathrm{C}(25)-\mathrm{C}(24)-\mathrm{H}(24) & 119.2 \\ \mathrm{C}(18)-\mathrm{C}(25)-\mathrm{C}(24) & 118.3(5) \\ \mathrm{C}(18)-\mathrm{C}(25)-\mathrm{C}(26) & 119.6(5) \\ \mathrm{C}(24)-\mathrm{C}(25)-\mathrm{C}(26) & 122.2(5) \\ \mathrm{C}(27)-\mathrm{C}(26)-\mathrm{C}(31) & 119.4(5) \\ \mathrm{C}(27)-\mathrm{C}(26)-\mathrm{C}(25) & 120.9(5) \\ \mathrm{C}(31)-\mathrm{C}(26)-\mathrm{C}(25) & 119.5(4) \\ \mathrm{C}(28)-\mathrm{C}(27)-\mathrm{C}(26) & 119.2(5) \\ \mathrm{C}(28)-\mathrm{C}(27)-\mathrm{H}(27) & 120.4 \\ \mathrm{C}(26)-\mathrm{C}(27)-\mathrm{H}(27) & 120.4 \\ \mathrm{C}(27)-\mathrm{C}(28)-\mathrm{F}(2) & 118.8(5) \\ \mathrm{C}(27)-\mathrm{C}(28)-\mathrm{C}(29) & 124.1(5) \\ \mathrm{F}(2)-\mathrm{C}(28)-\mathrm{C}(29) & 117.1(5) \\ \mathrm{C}(30)-\mathrm{C}(29)-\mathrm{C}(28) & 116.9(5) \\ \mathrm{C}(30)-\mathrm{C}(29)-\mathrm{H}(29) & 121.6 \\ \mathrm{C}(28)-\mathrm{C}(29)-\mathrm{H}(29) & 121.6 \\ \mathrm{C}(29)-\mathrm{C}(30)-\mathrm{C}(31) & 122.4(5) \\ \mathrm{C}(29)-\mathrm{C}(30)-\mathrm{H}(30) & 118.8 \\ \mathrm{C}(31)-\mathrm{C}(30)-\mathrm{H}(30) & 118.8 \\ \mathrm{C}(30)-\mathrm{C}(31)-\mathrm{C}(26) & 117.2(4) \\ \mathrm{C}(30)-\mathrm{C}(31)-\mathrm{C}(32) & 122.1(5) \\ \mathrm{C}(26)-\mathrm{C}(31)-\mathrm{C}(32) & 120.4(5) \\ \mathrm{C}(17)-\mathrm{C}(32)-\mathrm{C}(31) & 117.9(4) \\ \mathrm{C}(17)-\mathrm{C}(32)-\mathrm{C}(4) & 119.1(4) \\ \mathrm{C}(31)-\mathrm{C}(32)-\mathrm{C}(4) & 123.0(5) \\ & \end{array}$

Symmetry transformations used to generate equivalent atoms: 
Supporting Information

Table 4. Anisotropic displacement parameters $\left(\AA^{2} \times 10^{3}\right)$ for flu-dbc. The anisotropic displacement factor exponent takes the form: $-2 \pi^{2}\left[h^{2} a^{* 2} U^{11}+\ldots+2 h k a^{*} b^{*} U^{12}\right]$

\begin{tabular}{|c|c|c|c|c|c|c|}
\hline & $\mathrm{U}^{11}$ & $\mathrm{U}^{22}$ & $\mathrm{U}^{33}$ & $\mathrm{U}^{23}$ & $\mathrm{U}^{13}$ & $\mathrm{U}^{12}$ \\
\hline $\mathrm{C}(1)$ & $34(4)$ & $18(3)$ & 41(4) & $-7(2)$ & $-7(3)$ & $-3(3)$ \\
\hline$C(2)$ & $26(3)$ & $17(2)$ & $34(4)$ & $0(2)$ & $-2(3)$ & $2(2)$ \\
\hline$C(3)$ & $14(3)$ & $13(2)$ & $31(3)$ & $-1(2)$ & $-3(3)$ & $4(2)$ \\
\hline C(4) & $14(3)$ & $17(2)$ & $25(3)$ & $-2(2)$ & $-3(2)$ & $6(2)$ \\
\hline$C(5)$ & $31(3)$ & $20(2)$ & $27(3)$ & $2(2)$ & $4(3)$ & $4(3)$ \\
\hline $\mathrm{C}(6)$ & $30(3)$ & $23(3)$ & $30(3)$ & $-7(2)$ & $-1(3)$ & $3(2)$ \\
\hline$C(7)$ & $15(3)$ & $13(2)$ & $30(3)$ & $5(2)$ & $2(2)$ & $0(2)$ \\
\hline $\mathrm{C}(8)$ & $24(3)$ & $13(2)$ & $39(4)$ & $5(2)$ & $-3(3)$ & $-2(2)$ \\
\hline C(9) & $25(3)$ & 19(3) & $38(4)$ & $8(2)$ & $2(3)$ & $1(2)$ \\
\hline$C(10)$ & $21(3)$ & $17(2)$ & $26(3)$ & $8(2)$ & $0(3)$ & $2(2)$ \\
\hline $\mathrm{C}(11)$ & $24(3)$ & $27(3)$ & $34(4)$ & $8(2)$ & $3(3)$ & $4(3)$ \\
\hline $\mathrm{C}(12)$ & $24(3)$ & $34(3)$ & 21(3) & $6(2)$ & $-5(3)$ & $9(3)$ \\
\hline $\mathrm{C}(13)$ & 13(3) & $25(3)$ & $24(3)$ & 1(2) & $4(3)$ & $5(2)$ \\
\hline$C(14)$ & $8(3)$ & 19(3) & $28(3)$ & 2(2) & $0(2)$ & $1(2)$ \\
\hline$C(15)$ & $16(3)$ & $16(2)$ & $28(3)$ & $3(2)$ & 2(3) & $4(2)$ \\
\hline$C(16)$ & $13(3)$ & $11(2)$ & $27(3)$ & $2(2)$ & $1(2)$ & $-1(2)$ \\
\hline $\mathrm{C}(17)$ & $16(3)$ & $15(2)$ & 23(3) & $0(2)$ & $0(2)$ & $5(2)$ \\
\hline$C(18)$ & $12(3)$ & $10(2)$ & $32(3)$ & $0(2)$ & 1(3) & $-1(2)$ \\
\hline $\mathrm{C}(19)$ & $16(3)$ & $15(2)$ & $22(3)$ & $-1(2)$ & $4(2)$ & $4(2)$ \\
\hline$C(20)$ & $17(3)$ & $17(2)$ & $31(3)$ & $-6(2)$ & 1(3) & $4(2)$ \\
\hline$C(21)$ & $26(3)$ & $23(3)$ & $28(3)$ & $-10(2)$ & $-5(3)$ & 2(3) \\
\hline$C(22)$ & 21(3) & $33(3)$ & $26(3)$ & $-4(3)$ & $0(3)$ & $5(3)$ \\
\hline$C(23)$ & 21(3) & $16(2)$ & $38(4)$ & $-6(2)$ & $-3(3)$ & $1(2)$ \\
\hline$C(24)$ & $27(3)$ & $14(2)$ & $38(4)$ & $0(2)$ & $6(3)$ & $-3(2)$ \\
\hline$C(25)$ & $15(3)$ & $15(2)$ & 29(3) & 2(2) & $4(2)$ & $4(2)$ \\
\hline$C(26)$ & 21(3) & $18(2)$ & $30(3)$ & 1(2) & 2(3) & $2(2)$ \\
\hline $\mathrm{C}(27)$ & $23(3)$ & $13(2)$ & $35(4)$ & $-1(2)$ & $5(3)$ & $3(2)$ \\
\hline$C(28)$ & 41(4) & $15(2)$ & $29(3)$ & $9(2)$ & $14(3)$ & 1(3) \\
\hline $\mathrm{C}(29)$ & $29(3)$ & 19(2) & $22(3)$ & $6(2)$ & $9(3)$ & $5(2)$ \\
\hline $\mathrm{C}(30)$ & $23(3)$ & $18(2)$ & $31(3)$ & $-5(2)$ & $5(3)$ & $4(2)$ \\
\hline $\mathrm{C}(31)$ & 19(3) & $16(2)$ & $25(3)$ & $2(2)$ & $-1(3)$ & $3(2)$ \\
\hline $\mathrm{C}(32)$ & $24(3)$ & $14(2)$ & $28(3)$ & $2(2)$ & $-5(3)$ & $1(2)$ \\
\hline $\mathrm{F}(1)$ & $53(2)$ & $24(2)$ & $47(2)$ & $-13(1)$ & $-9(2)$ & $-7(2)$ \\
\hline $\mathrm{F}(2)$ & $68(3)$ & $23(2)$ & $34(2)$ & $12(1)$ & $8(2)$ & $-4(2)$ \\
\hline
\end{tabular}

